\title{
Conditional activation of NRG1 signaling in the brain modulates cortical circuitry
}

\author{
Dissertation \\ for the award of the degree \\ „Doctor rerum naturalium“ (Dr. rer. nat.) \\ Division of Mathematics and Natural Sciences \\ of the Georg-August-Universität Göttingen \\ Basic program Biology \\ of the Georg-August University School of Science (GAUSS) \\ submitted by \\ Tilmann Unterbarnscheidt \\ born in \\ Essen (an der Ruhr), Germany
}

Göttingen 2015 


\section{Ph.D. thesis committee:}

Prof. Klaus-Armin Nave, Ph.D. (Reviewer)

Department of Neurogenetics,

Max-Planck-Institute of Experimental Medicine Göttingen

Prof. Dr. Dr. Hannelore Ehrenreich (Reviewer)

Department of Clinical Neurosciences,

Max-Planck-Institute of Experimental Medicine Göttingen

Dr. Dieter Klopfenstein (only Ph.D. thesis comittee)

Department of Biophysics,

Third Institute of Physics, Georg-August-Universität Göttingen

Extended Ph.D. thesis committee:

Prof. Dr. Martin Göpfert

Department of Cellular Neurobiology,

Schwann-Schleiden Research Centre, Georg-August-Universität Göttingen

Dr. Markus H. Schwab

Department of Neurogenetics,

Max-Planck-Institute of Experimental Medicine Göttingen

Present address: Cellular Neurophysiology, Hannover Medical School

Prof. Dr. Nils Brose

Department of Molecular Neurobiology,

Max-Planck-Institute of Experimental Medicine Göttingen

Prof. Dr. Ralf Heinrich

Department of Cellular Neurobiology,

Schwann-Schleiden Research Centre, Georg-August-Universität Göttingen

Date of the oral examination: 05.05.2015 
The present study was performed from february 2012 to april 2015 at the Max-Planck-Institute of Experimental Medicine (Göttingen) in the Department of Neurogenetics under guidance of Prof. Klaus-Armin Nave, Ph.D. and supervision of Dr. Markus H. Schwab 


\section{Declaration}

I hereby declare that I prepared the Ph.D. thesis entitled: "Conditional activation of NRG1 signaling in the brain modulates cortical circuitry", on my own and with no other sources and aids than quoted.

The first chapter of my result section is modified from our manuscript "Dysregulated Expression of Neuregulin-1 by Cortical Pyramidal Neurons Disrupts Synaptic Plasticity", published in the journal Cell Reports in 2014, and primarily reflects my contribution to the manuscript.

Göttingen, April 2015

Tilmann Unterbarnscheidt 
To my parents. 
I went to the woods because I wished to live deliberately. I wanted to live deep and suck out all the marrow of life, to live so sturdily and Spartan-like as to put to rout all that was not life. And not, when I came to die, discover that I had not lived.

Henry David Thoreau 


\section{Acknowledgments}

I am sincerely grateful to Dr. Markus Schwab for his supervision. I want to thank him for his support and believing in me for all these years. With his influence, I developed as a scientist.

I thank Prof. Klaus-Armin Nave Ph.D. for giving me the opportunity to work in his department for so many years. Being a member of the neurogenetics department has greatly influenced, challenged and improved my scientific work.

I thank the members of my thesis committee, Prof. Dr. Dr. Hannelore Ehrenreich and Dr. Dieter Klopfenstein, for helpful discussions in the committee meetings and their support during my graduation. I further thank the members of my extended thesis committee, Prof. Dr. Nils Brose and Prof. Dr. Ralf Heinrich for taken part in my final exams.

I thank Amit Agarwal Ph.D. and Dr. Payam Dibaj for a great collaboration on our manuscript and their influence to my work.

I thank Dr. Magdalena Brzózka for her help and support and teaching me how to conduct behavior experiments. I also thank Prof. Dr. Moritz Rossner for allowing me to use his behavior unit and work with his equipment.

I thank Prof. Dr. Evgeni Ponimaskin for the supply of antibodies.

I thank Dr. Christoph Biesemann and Dr. Ben Cooper for helpful discussions on synapses and sharing their knowledge and experiences with me.

I thank Dr. Ali Shahmoradi and Dr. Michael Wehr for helpful discussions and sharing their knowledge on western blotting with me.

I want to thank my current and former group members, especially Dr. Viktorija Velanac for her support and sharing her scientific experiences with me and becoming a real friend. I thank Maike Gummert for cloning of the Stop-cassette. I also want to thank my colleague María Clara Soto Bernardini for her help and support.

I want to thank Marcel Floerl, Annette Fahrenholz, Verena Meywirth, Ulrike Bode, Gudrun Fricke-Bode and Jenniffer Günther for technical support in many ways.

I thank the animal caretakers for looking after my mice, especially Tanja Freerck and Tanja Hoffmeister.

I want to thank Hans-Joachim Horn, Lothar Demel, Rolf Merker and Beate Beschke for technical help regarding computer and printing issues. 
I want to thank the whole neurogenetics department for the great working atmosphere. I especially want to thank my friends and colleagues Georg, Karim, Theresa, Ulrike G., Sarah, Lotti, Dorota, Wilko, Sven, Katrin, Jessica, Christina, Aiman, Iva, Andrea, Jan, Madlen, Matthäus, Torben and Susanne for their support and for making it a fun time in the lab.

I'm sincerely grateful for my wonderful family that has supported me all my life. Without them, this wouldn't have been possible.

I'm also grateful to all my friends for their support.

I want to especially thank Nadja Götze for her love, support, encouragement and for kicking my butt to the finish line. 


\section{Content}

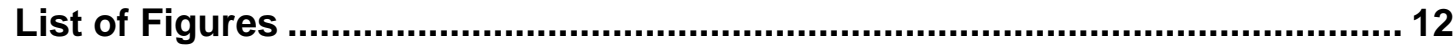

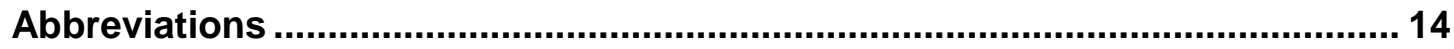

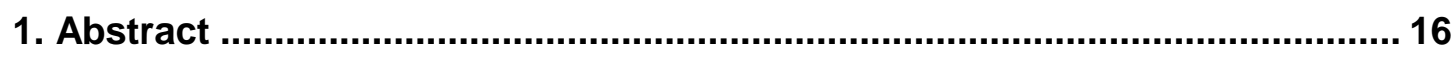

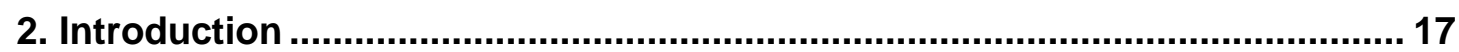

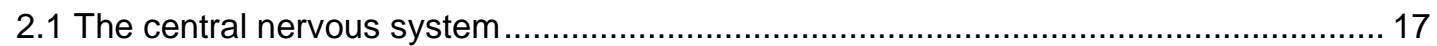

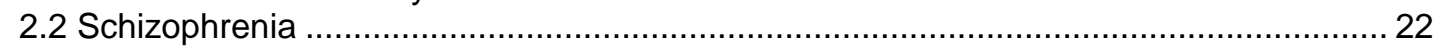

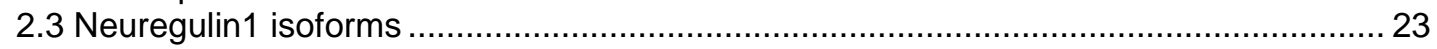

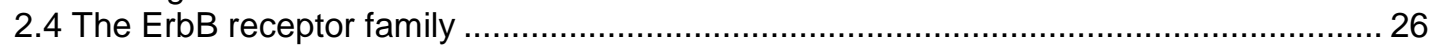

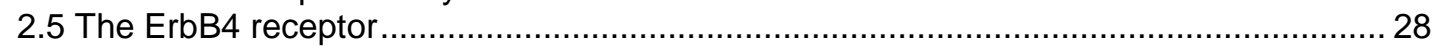

2.6 NRG1/ErbB4 signaling in cortical networks during health and disease ........................ 30

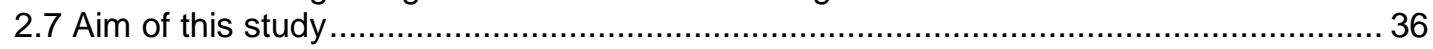

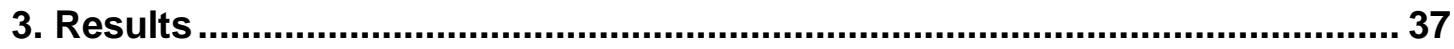

3.1 Consequences of NRG1 deficiency on interneuronal migration and integration ............ 37

3.2 Elevated CRD-NRG1 expression leads to sustained ErbB4 hyperstimulation, altered

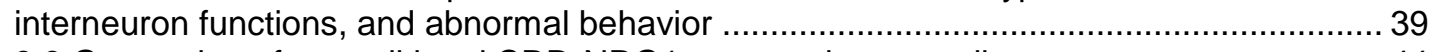

3.3 Generation of a conditional CRD-NRG1 transgenic mouse line ................................. 44

3.4 NEX-Cre mediated recombination of Stop-Nrg1 restricts HA-NRG1 expression to

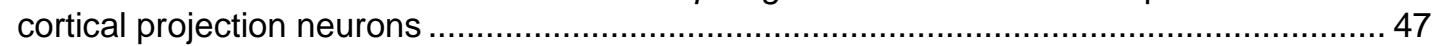

3.5 Stop-Nrg1*NEX-Cre mice model HA-NRG1 overexpression in the embryonic cortex ... 50

3.6 CamKII-Cre mice enable postnatal overexpression of HA-NRG1 in cortical projection

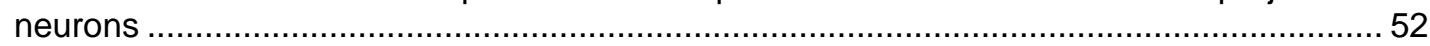

3.7 Absense of brain pathology in Stop-Nrg $1^{*} \mathrm{NEX}$-Cre and Stop-Nrg ${ }^{*} \mathrm{CKII}-\mathrm{Cre}$ mice ...... 54

3.8 Conditional CRD-NRG1 overexpression has no effect on interneuronal migration and

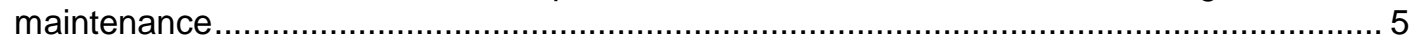

3.9 Cortical-restricted HA-NRG1 overexpression is not linked to ventricular enlargement

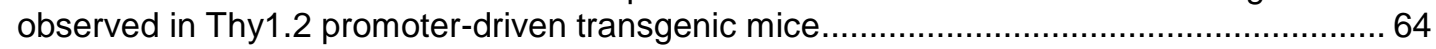

3.10 Thy1.2-driven NRG1 transgenic mice have reduced body weight .............................6 66

3.11 Stop-Nrg1 ${ }^{*} N E X$-Cre mice show no signs of anxiety-like behavior, decreased PPI or cognitive impairments, but exhibit hyperactivity ............................................................ 67 3.12 Postnatal cortical overexpression of HA-NRG1 in Stop-Nrg $1{ }^{*} \mathrm{CKII-Cre} \mathrm{mice} \mathrm{has} \mathrm{only}$ minor effects on behavior ...................................................................................... 72 3.13 Stop-Nrg ${ }^{*} N E X$-CreERT2 mice as a tool for acute overexpression of NRG1 in the adult

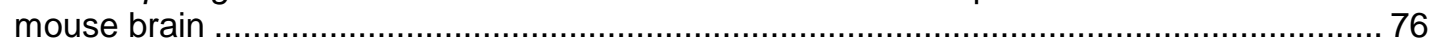
3.14 Biochemical analysis of CRD-NRG1 signaling in NRG1 transgenic mice ..................... 79

3.15 NRG1 forward and backsignaling - differential recruitment of LIMK1? ...................... 81

3.16 HA-NRG1 is enriched in synaptosomes of Stop-Nrg1*NEX-Cre mice and regulates

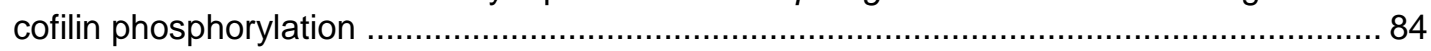

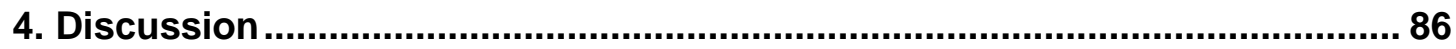

4.1 Embryonic NRG1 signaling is dispensable for interneuron migration ............................ 86

4.2 CRD-NRG1 overexpression hyperstimulates ErbB4 receptors and alters inhibitory

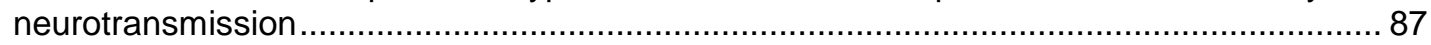

4.3 CRD-NRG1 transgenic mice show SZ-relevant behavioral dysfunctions ............................ 89

4.4 Conditional overexpression of CRD-NRG1 in transgenic mice ..................................... 89

4.5 Modeling stage-specific CRD-NRG1 overexpression in cortical projection neurons...... 90 4.6 Embryonic and postnatal HA-NRG1 overexpression is not associated with neuropathology or -inflammation at adult stages ........................................................... 91

4.7 Thy1.2-driven CRD-NRG1 mice have enlarged ventricles and reduced body weight.... 93 4.8 Embryonic HA-NRG1 overexpression induces hyperactivity, but no anxiety-like behavior

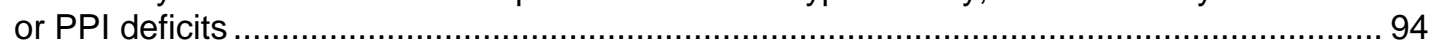

4.9 Stop-Nrg ${ }^{*} N E X$-CreERT2 mice allow acute NRG1 overexpression in the adult brain .. 97 4.10 Possible roles of NRG1-mediated forward and backsignaling in cortical network

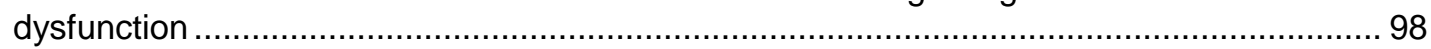

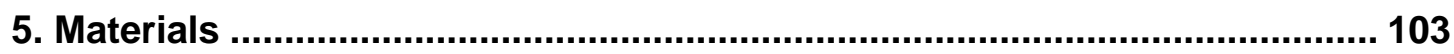

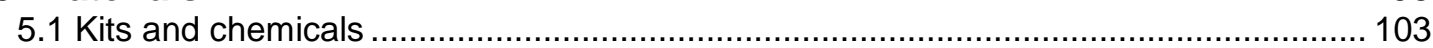




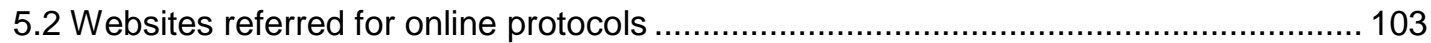

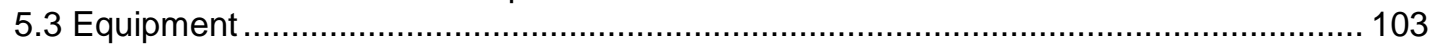

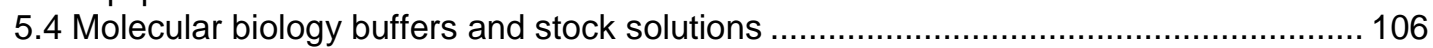

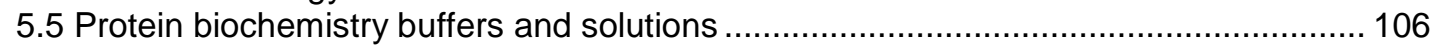

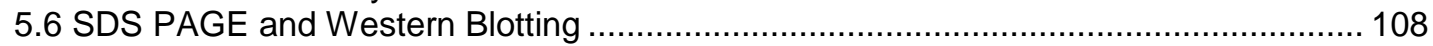

5.7 DNA and Protein markers ............................................................................ 111

5.8 Immunohistochemistry buffers and solutions ..................................................... 111

5.9 Buffers for immunohistochemisty of vibrotome and cryo sections .............................. 113

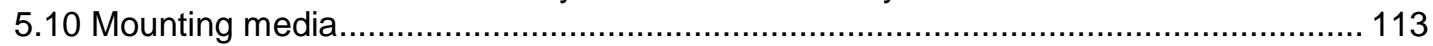

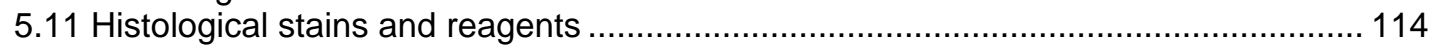

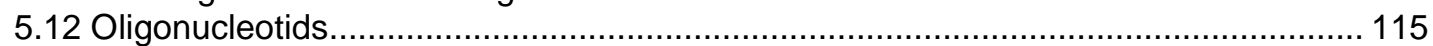

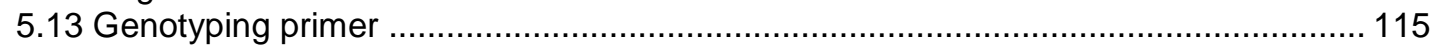

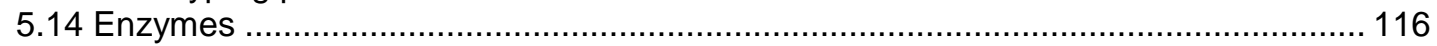

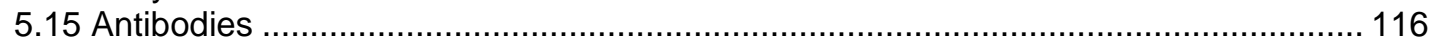

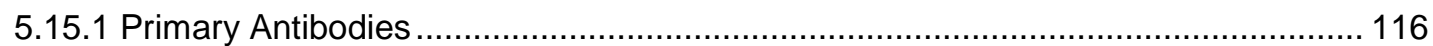

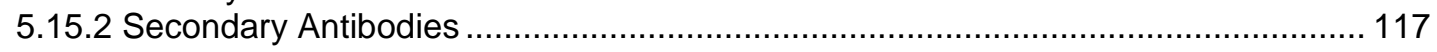

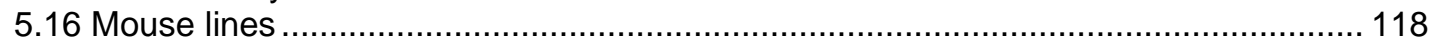

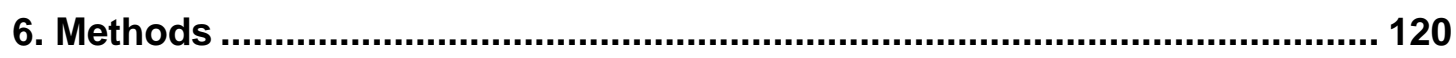

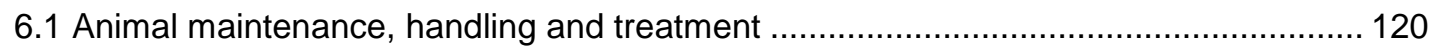

6.1.1 Generation of conditional Stop-Nrg1 transgenic mice ........................................... 120

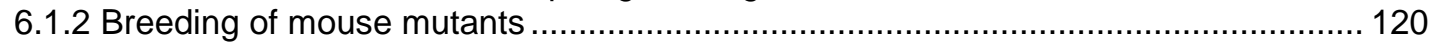

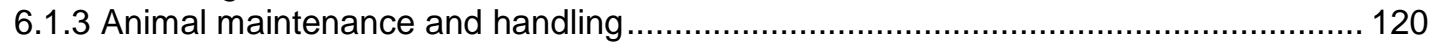

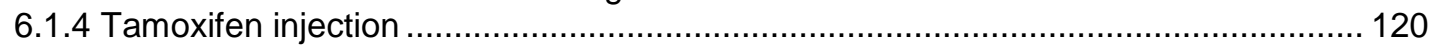

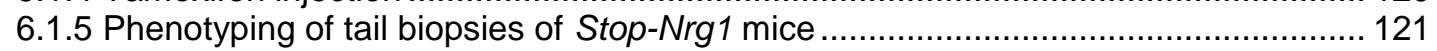

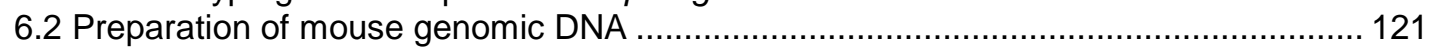

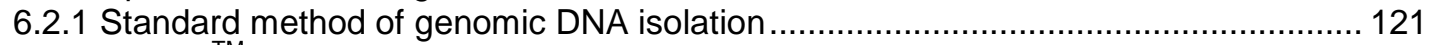

6.2.2 nexttec ${ }^{\text {TM }}$ Tissue \& Cells kit-based genomic DNA isolation ..................................... 121

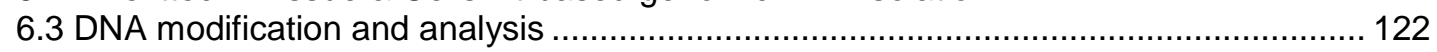

6.3.1 DNA amplification in vitro by polymerase chain reaction (PCR) ............................ 122

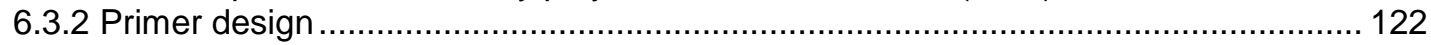

6.3.3 Agarose gel electorphoresis for the size separation of DNA fragments ................... 123

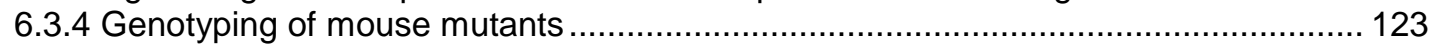

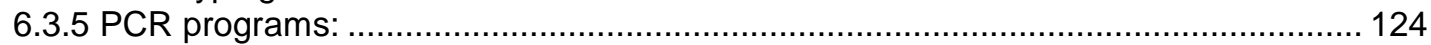

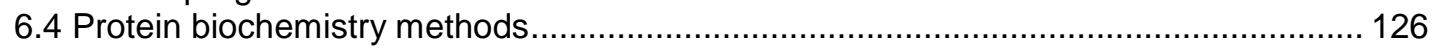

6.4.1 Radioimmunoprecipitation assay (RIPA) buffer protein extraction ......................... 126

6.4.2 Sucrose buffer protein extraction ................................................................. 126

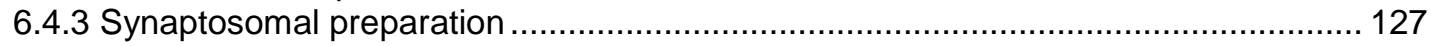

6.4.4 Protein concentration measurement with the Lowry assay .................................. 129

6.4.5 Preparation of protein samples for SDS electrophoresis ..................................... 129

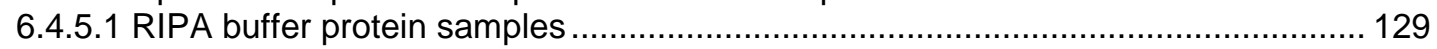

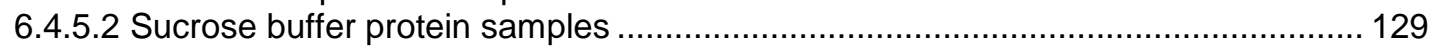

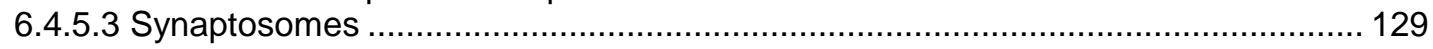

6.4.6 Discontinous SDS polyacrylamide gel electrophoresis ..................................... 130

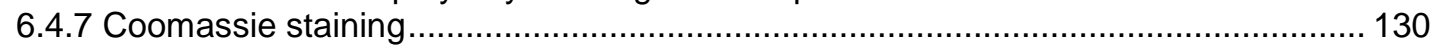

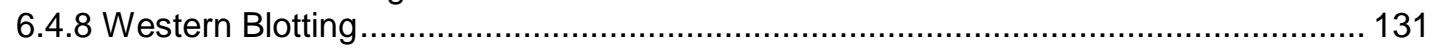

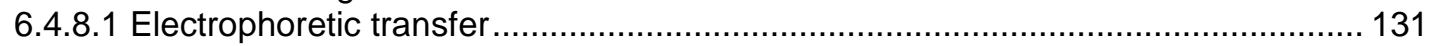

6.4.8.2 Immunological detection of proteins on PVDF membranes ................................ 131

6.4.8.3 Densitometric quantification of band intensity ............................................... 132

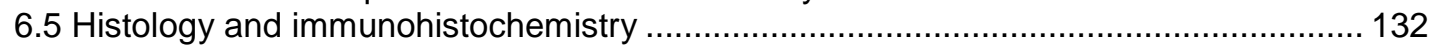

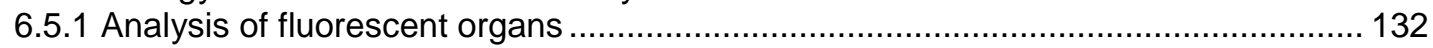

6.5.2 Perfusion fixation of mouse tissue for histology.............................................. 132

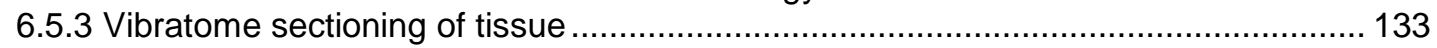

6.5.4 Cryo protection, embedding and cryosectioning of tissue .................................. 133

6.5.5 Paraplast impregnation, embedding and sectioning of tissue .............................. 134

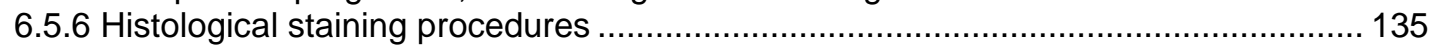

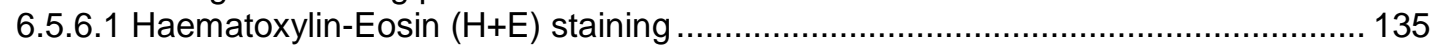

6.5.6.2 DAB immunostaining of paraffin sections ................................................... 135

6.5.6.3 DAB immunostaining of paraffin sections using the Ventana staining machine..... 136

6.5.6.4 Fluorescent immunostaining of paraffin sections ................................................ 136

6.5.6.5 Fluorescent staining of vibratome sections................................................... 137 
6.5.6.6 Fluorescent staining of cryo sections .......................................................... 137

6.5.6.7 Detection of $\beta$-galactosidase in tissue sections (X-Gal staining) ......................... 138

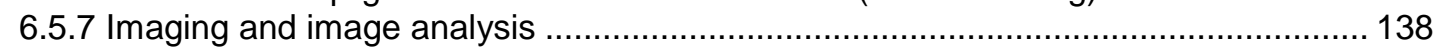

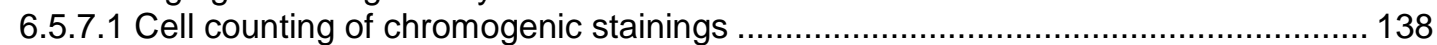

6.5.7.2 Quantification of $\mathrm{GFAP}^{+}$and IBA $1^{+}$areas in chromogenic stainings..................... 139

6.5.7.3 Two-Photon laser scanning microscopy .............................................................. 139

6.5.7.4 Data analysis and statistics...................................................................... 140

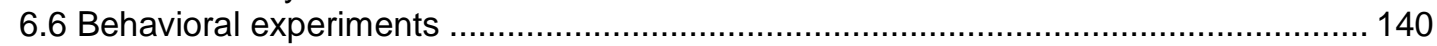

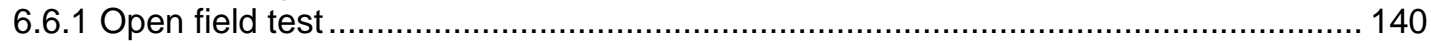

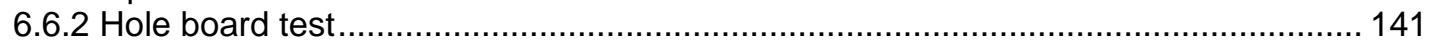

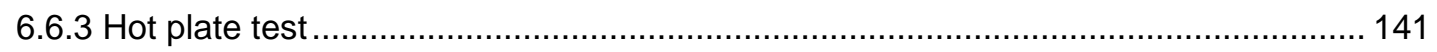

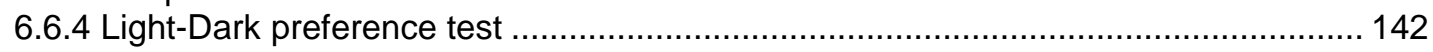

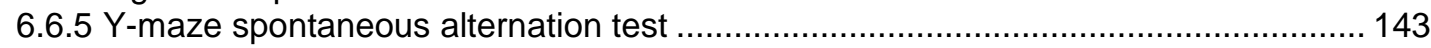

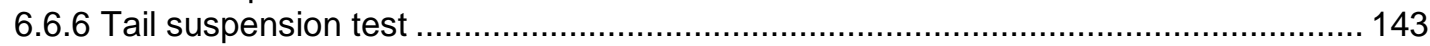

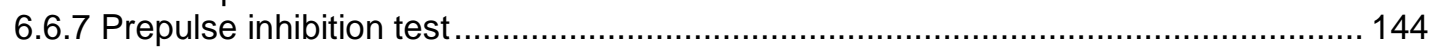

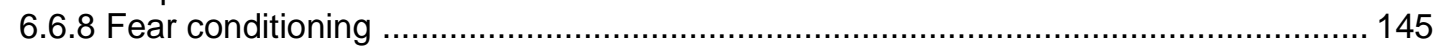

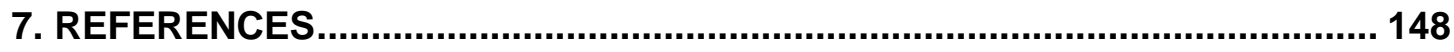

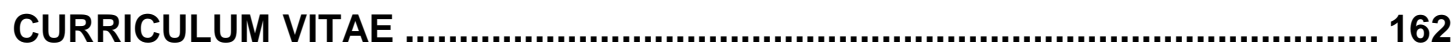

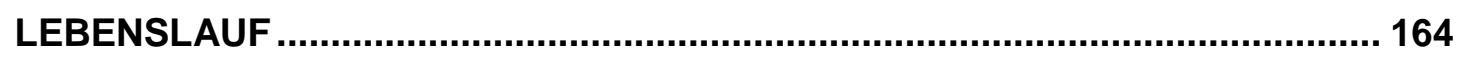




\section{List of Figures}

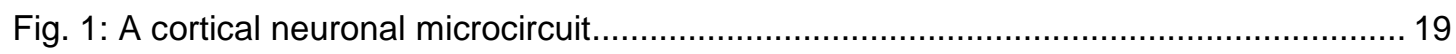

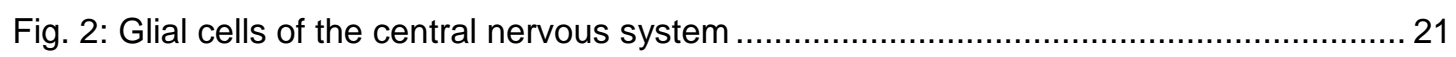

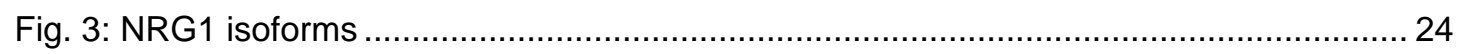

Fig. 4: ErbB receptors: ligand binding and receptor dimerization ....................................... 28

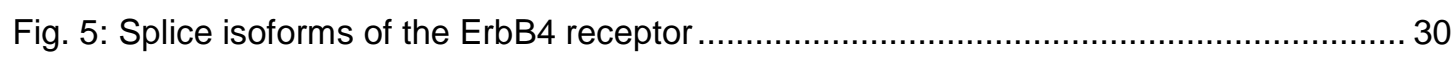

Fig. 6: Model of canonical and non-canonical NRG1/ErbB4 signaling................................. 34

Fig. 7: NRG1/ErbB4 signaling regulates various aspects of cortical networks. ...................... 35

Fig. 8: Embryonic NRG1 signaling is dispensable for cortex lamination and interneuronal migration

Fig. 9: Elevated CRD-NRG1 expression results in ErbB4 hyperphosphorylation and altered numbers of GABAergic interneurons

Fig. 10: HA-Nrg1-tg mice exhibit SZ-relevant behavior reduced numbers of interneurons in the cortex.....

Fig. 11: A $\beta$-actin GFP-Stop-flox transgene cassette allows Cre-mediated transgene expression

Fig. 12: Stop-Nrg1 mice express the $\beta$-actin GFP-Stop-flox transgene in virtually all tissues. 46

Fig. 13: The Stop-Nrg1 transgene is predominantly expressed in projection neurons 48

Fig. 14: NEX-Cre-mediated activation of Stop-Nrg1 restricts HA-NRG1 expression to cortical projection neurons

Fig. 15: NEX-Cre-mediated recombination of Stop-Nrg1 allows early embryonic over-expression of HA-NRG1.

Fig. 16: CKII-Cre-mediated activation of Stop-Nrg1 allows postnatal overexpression of HA-NRG1 in cortical projection neurons 53

Fig. 17: Stop-Nrg1 ${ }^{*} N E X$-Cre and Stop-Nrg $1{ }^{*} \mathrm{CKII}$-Cre mice show no signs of neuro-inflammation

Fig. 18: Stop-Nrg1 ${ }^{*} \mathrm{NEX}$-Cre and Stop-Nrg $1^{*} \mathrm{CKII}$-Cre mice show no signs of astrogliosis.... 57

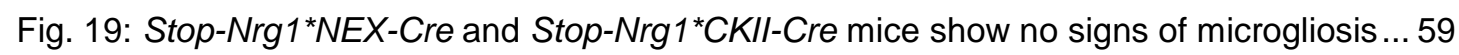

Fig. 20: NEX-Cre and Stop-Nrg1*NEX-Cre mice have reduced numbers of neurons

in the cortex at 1 year of age 60

Fig. 21: NEX-Cre mice show reduced numbers of GAD67 $7^{+}$interneurons in the cortex and hippocampus at 1 year of age.

Fig. 22: NEX-Cre and Stop-Nrg1*NEX-Cre mice have reduced numbers of $\mathrm{PV}^{+}$

interneurons in the cortex and hippocampus at one year of age 63

Fig. 23: Lateral ventricles are enlarged in HA-Nrg1-tg, but not in $\mathrm{HA}-\mathrm{Nrg} 1^{\mathrm{GIEF}}$ or conditional HA-NRG1 overexpressing mice 65

Fig. 24: 'Global' Nrg1-tg and HA-Nrg1-tg mice have reduced body weight 67

Fig. 25: Stop-Nrg1*NEX-Cre mice exhibit novelty-induced hyperactivity and increased fighting behavior in the tail suspension test 68 
Fig. 26: Stop-Nrg ${ }^{\star} N E X$-Cre mice show no impairments in working memory and sensorimotor gating, but exhibit reduced cued fear memory . 71

Fig. 27: Postnatal cortical overexpression of HA-NRG1 in Stop-Nrg $1{ }^{*} \mathrm{CKII-Cre} \mathrm{mice}$ has only minor effects on activity 73

Fig. 28: Stop-Nrg $1^{*} \mathrm{CKII}-\mathrm{Cre}$ mice exhibit normal learning and memory as well as sensorimotor gating 75

Fig. 29: Stop-Nrg ${ }^{*} N E X$-CreERT2 mice are a tool for tamoxifen-induced acute HA-NRG1 overexpression in adult mice and in vivo single-cell genetics 77

Fig. 30: NRG1 expression levels correlate with levels of ErbB4 hyperphosphorylation 80

Fig. 31: A comparative analysis of NRG1 transgenic mouse models reveals differences in forward and backsignaling 83

Fig. 32: HA-NRG1 is enriched in synaptosomes of Stop-Nrg1*NEX-Cremice and regulates cofilin phosphorylation... 85

Fig. 33: Preparation of crude synaptosomal fractions ...... 128

Fig. 34: Bio-Rad Mini Trans-Blot western blot sandwich. 131

Fig. 35: TSE Systems open field and hole board system. 141

Fig. 36: Hot plate test setup 142

Fig. 37: Light-Dark preference test setup 142

Fig. 38: Y-maze spontaneous alternation test setup.... 143

Fig. 39: Tail suspension test 144

Fig. 40: San Diego Instruments' SR-Lab startle response system 145

Fig. 41: Ugo Basile Series 46000 and TSE Systems fear conditioning setups. 147 


\section{Abbreviations}

\begin{tabular}{|c|c|}
\hline AMPA & $\alpha$-amino-3-hydroxy-5-methyl-4-isoxazolepropionic acid \\
\hline AKT & Protein kinase B \\
\hline APP & Amyloid beta precursor protein \\
\hline $\mathrm{bp}$ & Base pairs \\
\hline${ }^{\circ} \mathrm{C}$ & Degrees Celsius (centigrades) \\
\hline $\mathrm{CA}$ & Cornu Ammonis \\
\hline CamKII & Calcium/calmodulin-dependent protein kinase II \\
\hline ChaT & Choline acetyltransferase \\
\hline $\mathrm{DAB}$ & 3,3'-Diaminobenzidine \\
\hline DAPI & 4'-6-Diamidino-2-phenylindole \\
\hline$d B$ & Decibel \\
\hline $\mathrm{dd}_{2} \mathrm{O}$ & Double distilled (or miliQ) water \\
\hline DMSO & Dimethylsulfoxide \\
\hline DNA & Deoxyribonucleic acid \\
\hline DTT & Dithiothreitol \\
\hline EDTA & Ethylened acid \\
\hline e.g. & Exempli gratia \\
\hline ERK & Extracellular signal-regulated kinase \\
\hline f.c. & Final concentration \\
\hline$g$ & Gram \\
\hline GAD67 & Glutamate-Decarboxylase of $67 \mathrm{kDa}$ \\
\hline GFAP & Glial fibrillary acidic protein \\
\hline GFP & Green fluorescent protein \\
\hline $\mathrm{h}$ & Hours \\
\hline HA-tag & Human influenza hemagglutinin tag \\
\hline HEPES & 4-(2-hydroxyethyl)-1-piperazineethanesulfonic acid \\
\hline Hipp & Hippocampus \\
\hline HRP & Horseradish peroxidase \\
\hline IBA1 & Ionized calcium-binding adapter molecule 1 \\
\hline i.e. & Id est \\
\hline $\mathrm{kb}$ & Kilobases \\
\hline $\mathrm{kg}$ & Kilogram \\
\hline $\mathrm{kDa}$ & Kilodalton \\
\hline $\mathrm{kHz}$ & Kilohertz \\
\hline I & Liter \\
\hline M & Molar \\
\hline$\mu \mathrm{M}$ & Micromolar \\
\hline $\mathrm{mM}$ & Millimolar \\
\hline $\mathrm{mm}$ & Millimeter \\
\hline $\mathrm{mA}$ & Milliampere \\
\hline MAPK & Mitogen-activated protein kinase \\
\hline MBP & Myelin basic protein \\
\hline $\min$ & Minutes \\
\hline $\mathrm{ml}$ & Milliliter \\
\hline $\mathrm{ms}$ & Milliseconds \\
\hline$\mu g$ & Microgram \\
\hline
\end{tabular}




$\begin{array}{ll}\mu l & \text { Microliter } \\ \text { ng } & \text { Nanogram } \\ \text { NMDA } & \text { N-Methyl-D-aspartic acid } \\ \text { n.s. } & \text { Non-significant } \\ \mu m & \text { Micrometer } \\ \text { nm } & \text { Nanometer } \\ \text { ON } & \text { Over night } \\ \text { p } & \text { P-value } \\ \text { P } & \text { Postnatal day } \\ \text { PCR } & \text { Polymerase chain reaction } \\ \text { pM } & \text { Picomole } \\ \text { PNS } & \text { Peripheral nervous system } \\ \text { PVDF } & \text { Polyvinylidene fluoride } \\ \text { Rpm } & \text { Revolutions per minute } \\ \text { RT } & \text { Room temperature } \\ \text { s (sec) } & \text { Seconds } \\ \text { SEM } & \text { Standard error of the mean } \\ \text { SZ } & \text { Schizophrenia } \\ \text { TEMED } & \text { Tetramethylendiamin } \\ \text { w/v } & \text { Weight per volume } \\ \text { U } & \text { Unit (for enzyme activities) } \\ \text { UV } & \text { Ultraviolet } \\ \text { V } & \text { Volt } \\ \text { v/v } & \text { Volume per volume } \\ \text { w/v } & \text { Weight per volume } \\ \text { xg } & \text { g = 9.81 ms }{ }^{-1}, \text { relative centrifugal force } \\ \text { X-gal } & \text { 5-bromo4-chloro-3-indolyl- } \beta \text {-D-galactoside }\end{array}$




\section{Abstract}

Neuregulin (NRG) 1 contains an epidermal growth factor (EGF)-like signaling domain and serves as a ligand for receptor tyrosine kinases of the ErbB family. ErbB4, the main neuronal NRG1 receptor in the brain, is enriched in GABAergic interneurons. NRG1/ErbB4 signaling regulates different aspects of nervous system development and synaptic plasticity in the mature brain. Variants of the human NRG1 and ERBB4 genes are genetic risk factors for schizophrenia, and inhibitory network dysfunctions have been implicated in schizophrenia. For NRG1 most of the at-risk haplotypes are located in non-coding regions, implicating that expression of NRG1 isoforms might be altered in SZ. In line with this, increased NRG1 expression and ErbB4 hyperphosphorylation was observed in postmortem brains of schizophrenia patients, suggesting that NRG1/ErbB4 hyperstimulation represents a possible pathomechanisms in schizophrenia. To test this hypothesis several NRG1 loss- and gain-of-function mouse models were employed to examine effects of altered NRG1 signaling on nervous system development and adult brain functions.

Conditional ablation of NRG1 in the embryonic cortex (Emx-Cre* Nrg $1^{\text {t/f }}$ mice) had no effect on cortical development, whereas pan-neuronal overexpression of the CRDNRG1 isoform in transgenic mice lead to ErbB4 hyperactivation, altered numbers of cortical interneurons, changes in dendritic spine morphology, ventricular enlargement, increased anxiety-like behavior, and impaired sensorimotor gating. These data suggest that the human NRG1 risk haplotypes exert a gain-of-function effect.

To study NRG1/ErbB4 hyperstimulation in a more selective in vivo model, a ,conditional' transgenic mouse line (Stop-Nrg1) was generated, which allows Cre recombinase-mediated CRD-NRG1 overexpression. This mouse line was examined in combination with different Cre ,driver' lines to model distinct temporal and spatial aspects of CRD-NRG1 overexpression in the brain. Postnatal onset of CRD-NRG1 overexpression had only minor effects on nervous system development and behavior, whereas early embryonic onset of NRG1 hyperstimulation lead to hyperactivity, consistent with the neurodevelopmental hypothesis of schizophrenia. Cortical-restricted CRD-NRG1 overexpression had no effect on ventricular size or sensorimotor gating, indicating functions of CRD-NRG1 signaling in subcortical networks. Finally, CRD-NRG1 was present in synaptosomal fractions and appears to recruit LIMK1-cofilin signaling, providing a potential mechanism for the regulation of dendritic spine dynamics. 


\section{Introduction}

\subsection{The central nervous system}

The human brain is the most complex biological structure on earth. It performs a large variety of complex tasks, including the reception, processing, integration and storage of information. In addition, by controlling the central and peripheral nervous system it controls and adapts body functions and motor behavior.

In the beginning of the 20th century Santiago Ramón y Cajal layed the foundation for our understanding of the nervous system by describing its central information processing unit, the neuron. The human brain consists of approximately $10^{11}$ neurons (Brose, 1999). These neurons are electrically excitable cells that form neuronal networks (Fig. 1). In order to achieve higher brain functions, neurons communicate with each other via two kinds of elaborations: multiple dendrites, which receive incoming informations and send them to the cell soma, and a single axon that passes electrical informations on to the next cell.

If the electrical excitation of a neuron reaches a certain threshold, an action potential is generated at the axon hillock and travels along the axon and eventually reaches a nerve terminal, the synapse. Neurons communicate via $10^{14}$ to $10^{15}$ of these synapses (Brose, 1999). At the synapse the plasma membranes of two cells come into close proximity and form a synaptic cleft. Both, the pre- and the postsynaptic membrane are specialized to transmit information from one cell to another. Neurons communicate with each other through electrical and chemical signals. Besides rare electrical synapses, the majority of neurons in the vertebrate central nervous system (CNS) communicate via chemical synapses. During neuronal communication an action potential that reaches a synapse triggers the fusion of synaptic vesicles with the presynaptic membrane. These vesicles contain neurotransmitters, that are released into the synaptic cleft and thereby transform electrical information into chemical signals. Neurotransmitters bind to receptors in the postsynapse. Depending on the neurotransmitter released and the neurotransmitter receptor it binds to, different postsynaptic reactions are triggered. Two kinds of neurotransmitter receptors exist in the nervous system, ligand-gated (ionotropic) receptors and Gprotein-coupled (metabotropic) receptors. Ionotropic receptors can either be excited, by neurotransmitters like glutamate or aspartate, or inhibited by ligands like GABA or glycine. Metabotropic receptors are not directly channel-linked. Upon ligand binding a conformational change is induced, which allows the receptor to activate adapter molecules, so called G-proteins, by exchanging their bond GDP by GTP. Thereupon, the G-protein dissociates and effects intracellular signaling cascades, that ultimately 
lead to ion channel opening or altered gene expression, and therefore can induce long-term changes in the activated cell (Kandel, 2013).

Cortical networks comprise two classes of neurons: excitatory (mostly glutamatergic) pyramidal neurons and inhibitory (mostly GABAergic) interneurons (Fig. 1). Pyramidal neurons process sensory or motor informations and generate output signals, that control other parts of the nervous system and body, including muscles in the periphery. Interneurons connect locally between neurons, are typically inhibitory and modulate the activity of pyramidal neurons by finetuning their excitability. In line with their different function, pyramidal neurons and interneurons also originate from different brain regions and progenitor cells. While excitatory neurons derive from progenitors in the ventricular zone (VZ) of the pallium and migrate radially into the emerging neocortex, inhibitory neurons originate from several progenitor pools in the subpallium, from where they migrate along tangential routes to the developing neocortex, where they shift from tangential to radial migration and invade different neocortical cell layers (Marin, 2013; Marin and Müller, 2014). GABAergic interneurons can be classified into nearly 30 different subtypes based on molecular, morphological and physiological criteria (DeFelipe et al., 2013). Since they originate from distinct progenitor pools and adopt their final cortical position following specific rules, interneuronal migration is a highly complex process (Marin, 2013).

Pyramidal neurons possess two kinds of dendrites. Basal dendrites elaborate from the side of the cell body from which also the axon originates. Apical dendrites originate from the opposite side. Dendrites of pyramidal neurons possess specific synaptic microdomains, the so-called dendritic spines, at which excitatory synapses terminate (Fig. 1). Dendritic spines are equipped with a postsynaptic density (PSD), which contains neurotransmitter receptors, ion channels and enzymes that serve in synaptic neurotransmission (Kennedy, 1997; Ziff, 1997). PSD95 (postsynaptic density protein of $95 \mathrm{kDa}$ ) is an important component of the PSD, involved in the molecular organisation of the postsynaptic complex. Pyramidal neurons form synapses with both other excitatory (glutamatergic) pyramidal neurons and inhibitory (GABAergic) interneurons (Markram et al., 2004). Excitatory synapses on pyramidal neurons are usually formed with dendritic spines. Inhibitory interneuron dendrites usually do not form dendritic spines and excitatory synapses on interneurons are less studied, however they are also equipped with a PSD. 


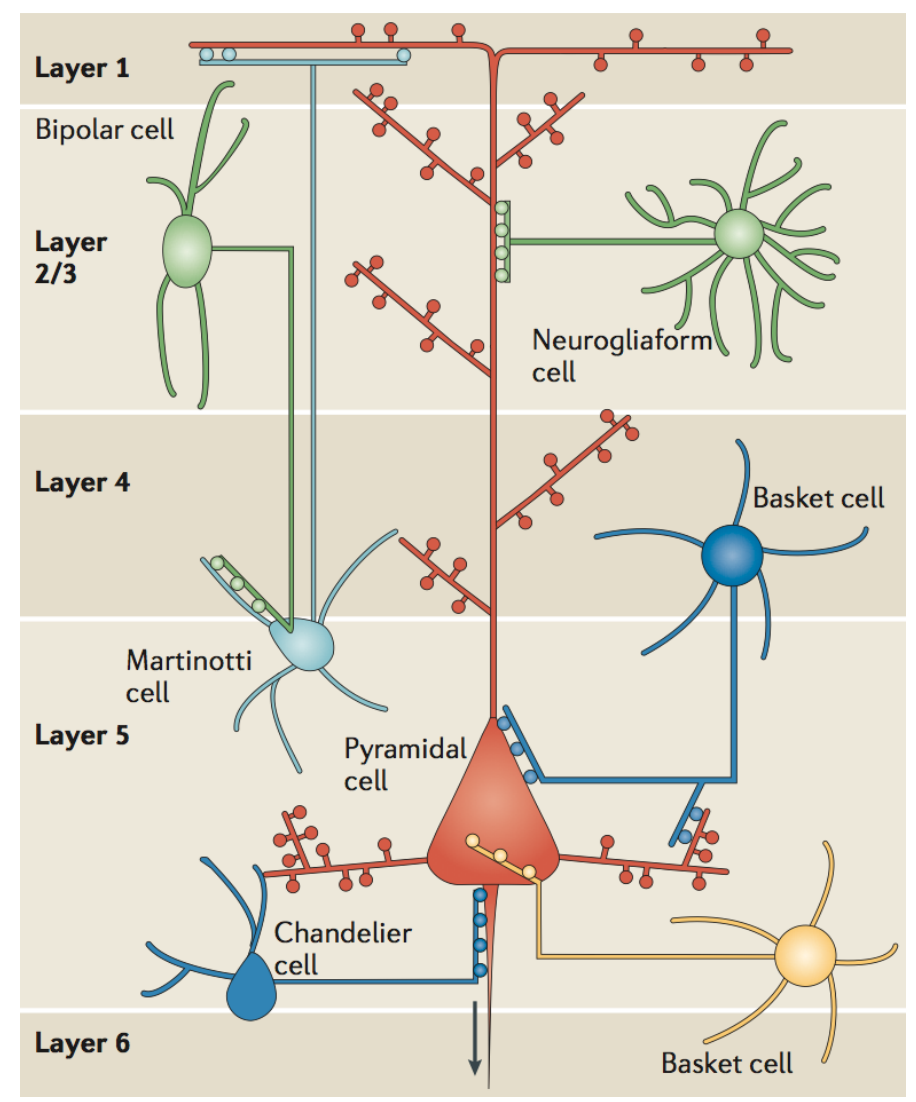

Fig. 1: A cortical neuronal microcircuit. A central excitatory pyramidal neuron is innervated by different classes of interneurons. Basket cells target the soma and basal dendrites of pyramidal cells, whereas chandelier cells synapse on the axon initial segment (AIS). Martinotti and neurogliaform cells types contact pyramidal cell dendrites. Bipolar cells are specialized in targeting other interneurons (modified from Marin, 2012).

About $20-30 \%$ of neocortical neurons are inhibitory interneurons. They are morphologically diverse and their precise classification is subject to ongoing discussion (see Fig. 1 and DeFelipe et al., 2013). Inhibitory interneurons use GABA as their main neurotransmitter. Inhibitory synapses to pyramidal neurons are usually formed with dendrites, the soma and the axonal initial segment (Freund and Buzsáki, 1996; Benes and Berretta, 2001) and constitute up to $16 \%$ of all synapses on cortical pyramidal neurons (Markram et al., 2004). Interneurons usually have smooth dendrites, without spines, and they receive excitatory and inhibitory synapses to their cell soma. Basket cells for instance usually form synapses with the perisomatic regions of pyramidal cells (Markram et al., 2004), whereas so-called „Chandelier"cells innervate the axonal initial segment and the axon hillock (Somogyi, 1977). "Neurogliaform" and „double-bouquet"-cells on the other hand form synapses with dendrites of pyramidal cells (see Fig. 1 and Benes and Berretta, 2001; Markram et al., 2004). Apart from these innervation profiles, interneurons can also be classified via their molecular properties, the expression of certain marker proteins, usually calcium-binding proteins, such as parvalbumin (PV), calretinin or calbindin, but also 
neuropeptides, including somatostatin, cholecystokinin (CCK), neuropeptide $Y$ (NPY), and vasoactive intestinal peptide (VIP) (Freund and Buzsáki, 1996; DeFelipe et al., 2013). A newly imerging criterium is the origin of interneuron subpopulations, whether they originate from the medial ganglionic eminence (MGE), lateral and dorsocaudal ganglionic eminence (CGE) or the preoptic area (POA). Yet another is the classification via electrical properties. Interneurons exhibit a variety of different firing patterns, including "fast-spiking" (FS) and "Non-adapting, non-fast-spiking" (NANFS) cells (reviewed in DeFelipe et al., 2013). Parvalbumin-positive ( $\left.\mathrm{PV}^{+}\right)$ interneurons for instance are usually fast-spiking interneurons that play an important role in the synchronization of pyramidal cell activity and the generation of $\gamma$ oszillations (Bartos et al., 2007).

Besides neurons, the nervous system consists of another, even more abundant cell type, the glial cells (Fig. 2). In fact glial cells outnumber neurons by 10 to 15 times (Kandel, 2013). The name glia comes from the Greek word for glue, because these cells were first thought to glue together nervous tissue. In addition to the stabilizing function, glial cells have been shown to provide several important functions, including myelination and metabolic support of axons.

Glial cells can be subdivided into two different cell classes, micro- and macroglia. While microglia are specialized macrophages, that are mobile, serve immune functions and protect the nervous system, CNS macroglia can be further divided into oligodendrocytes and astrocytes (Fig. 2).

The main function of oligodendrocytes is the insulation of axons of the central nervous system. By enwrapping axons with multiple layers of extended plasma membrane they produce a densly packed insulating sheath, the so-called myelin sheath (Fig. 2A). Because voltage-dependent sodium channels are only present at the nodes of ranvier, action potentials jump from node to node, in saltatory impulse propagation, increasing propagation speed by $\sim 100$-fold and reducing space and energy consumption (Garbay et al., 2000; Salzer, 2003). Unlike Schwann cells in the PNS, one oligodendrocyte can myelinate multiple axonal segments (Fig. 2A). Recent results also suggest a metabolic support function for axons, that is required for functional integrity and long-term survival (Funfschilling et al., 2012; Saab et al., 2013).

Astrocytes are named after the Greek word for star (,astron') because of their numerous projections giving them a star shape and allow them to connect to blood vessels and other cells. They are the most abundant cell type in the human brain and support endothelial cells in building the blood-brain barrier. Thereby they provide 
nutrients to the nervous tissue and also regulate the external chemical environment by removing excess ions, especially $\mathrm{K}^{+}$ions. Perinodal astrocytes contact the nodes of ranvier of CNS myelin, where they are thought to buffer the extracellular ion concentrations, but also stabilize the nodes and provide nutrients (see Fig. 2A and Black and Waxman, 1988). According to the tripartite synapse theory astrocytic processes engulf neuronal synapses and not only recycle neurotransmitters, but are also thought to modulate synaptic efficacy by release of "gliotransmitters", like glutamate or ATP (see Fig. 2B and Lalo et al., 2009; Santello and Volterra, 2009).
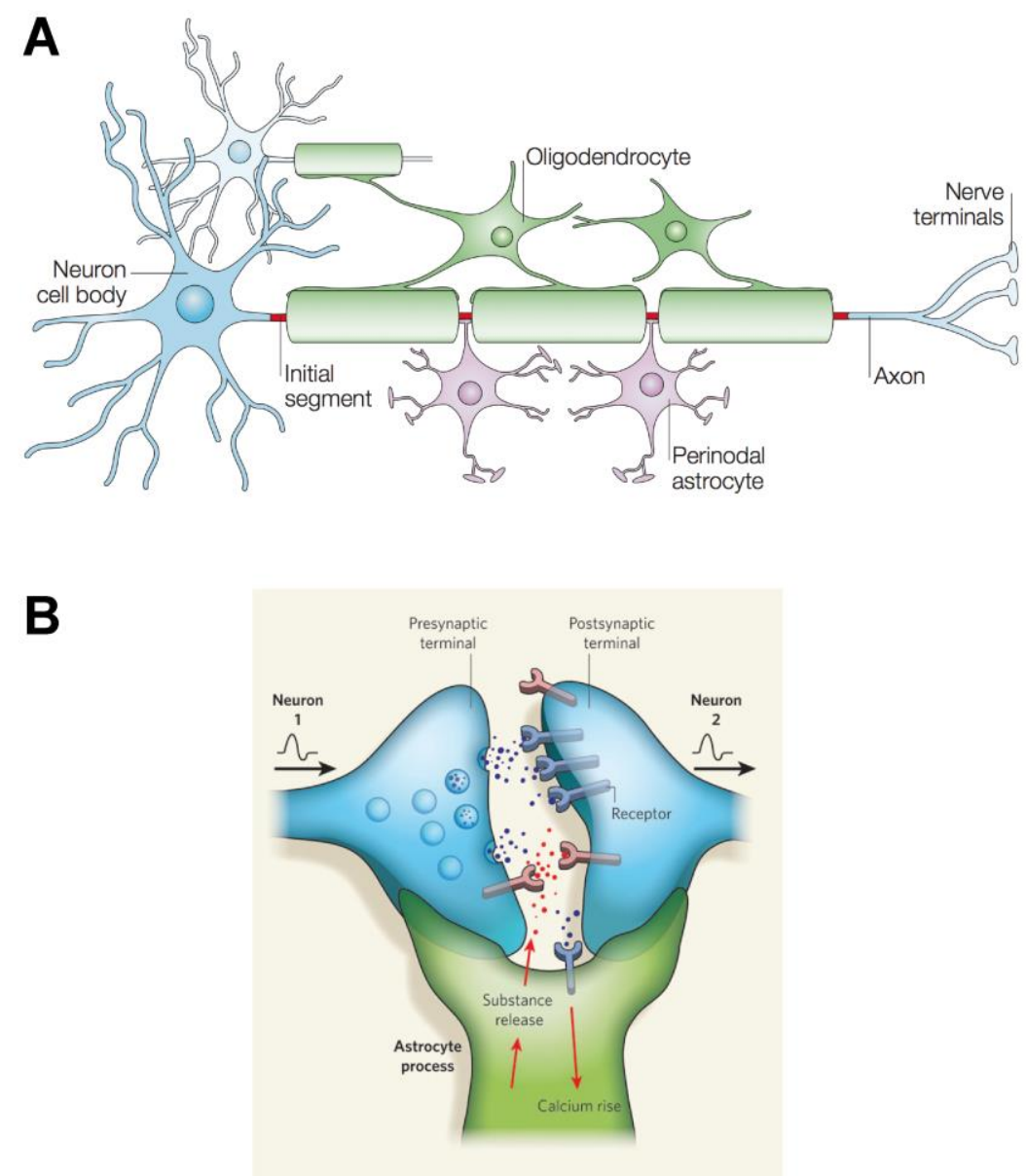

Fig. 2: Glial cells of the central nervous system. (A) Oligodendrocytes are the myelin forming cells of the CNS. In contrast to Schwann cells in the PNS, oligodendrocytes engulf multiple axonal segments with a myelin sheath. The myelin sheath speeds up neuronal signal propagation by saltatory impulse propagation. Perinodal astrocytes contact the nodes of ranvier, where they buffer the extracellular ion concentrations, stabilize the nodes and provide nutrients (modified from Poliak and Peles, 2003). (B) Astrocyte processes engulf neuronal synapses and form the so-called tripartite synapse. Astrocytes recycle neurotransmitters and modulate synaptic efficacy by releasing "gliotransmitters" (modified from Allen and Barres, 2009).

Taken together the central nervous system is a highly complex and dynamic structure, which comprises multiple cell types. By cell-cell communication the nervous system can dynamically adapt and rewire, for instance during learning when 
new synaptic connections are formed or some axonal pathways are strengthened, while others are retracted during development.

Apart from electrical and neurotransmitter-mediated neuronal communication, communication modules, consisting of ligands and receptors, regulate essential processes of neuronal development and maintenance. These modules are potentially involved in functions like neuronal migration, synapse formation and cortical network establishment and myelination. Defects in these signaling modules can result in impaired neuronal development and abnormalities in synaptic signaling, dysconnectivity, neuroinflammation and -degeneration. Ultimately, these processes can precipitate neurodegenerative diseases, e.g. Alzheimers disease or neuropsychiatric disorders such as schizophrenia.

\subsection{Schizophrenia}

Schizophrenia (SZ) is a devastating mental illness that affects an estimated 0.5 to 1 $\%$ of the general population world wide. The estimated financial burden to the European health care systems is $\sim 30$ billion $€$ annually. SZ has been considered a neurodegenerative disorder by some, but the most established data suggest that SZ is a late onset neurodevelopmental disorder. The age of onset of the disease is usually in late adolescence or during early adulthood. Data suggest that $S Z$ is affecting glutamatergic, dopaminergic and GABAergic cortical microcircuits directly or indirectly, as well as grey and white matter (Harrison and Weinberger, 2005; Owen et al., 2005). No clear causal genetic, biochemical or pathophysiological relationships have been identified yet. Thus, a clear classification of the disease by hallmark biomarkers is not possible. The diagnoses of SZ is based on characterization of behavioral phenotypes of patients (Andreasen, 1995; McKenna, 2013). These psychological and cognitive impairments can be grouped into positive (delusions, hallucinations and thought disorder) and negative symptoms (social withdrawal, cognitive deficits) (Harrison, 1999; McGuffin et al., 2004). Problems of establishing the validity and boundaries of SZ diagnosis, as in any syndromal diagnosis, exist, impacting on research regarding its pathological basis and causative genes (Jablensky, 1995; Kennedy, 1996). Although a clear cause of SZ is lacking, twin studies suggest a significant genetic component based on a $\sim 50 \%$ concordance in monozygotic twins. These studies also showed that SZ has a $\sim 80 \%$ heritability (Gottesman, 1991; McGuffin et al., 2004). In addition to a genetic predisposition, exposure to environmental factors seems to impact greatly on developing SZ. 
Environmental stressors that can occur in early development, during pregnancy or childhood, include for instance social stress or drug abuse. During adolescence, environmental factors can further damage the brain (Leask, 2004).

A number of morphological abnormalities have been identified in schizophrenia, including ventricular enlargement and reduced cerebral (cortical and hippocampal) volume. In addition alterations in synaptic, dendritic and axonal organization have been observed. Advances in microscopy enabled extensive studies of dendritic and axonal structures as well as synapses in postmortem tissue of SZ patients. These studies have shown abnormalities in neuronal processes and reduction of dendritic spines in SZ tissue (Moyer et al., 2014), suggesting SZ to be a synaptic disorder. These structural changes are supported by functional studies. Aberrant activity in the components of cortical circuits, involving the prefrontal cortex (PFC), the hippocampus and certain subcortical structures, like the dorsal thalamus, has been demonstrated through functional imaging data. Neuronal dysconnectivity between aforementioned brain regions is thought to result from functional abnormalities (Harrison, 1999).

Genome-wide association studies (GWAS) have identified multiple candidate genetic risk factors for SZ (>100). Alongside the ,classic' SZ at-risk locus disrupted in schizophrenia-1 (DISC1), the Neuregulin (NRG) 1 gene has emerged as a major risk gene for SZ (Harrison, 2015). NRG1 and its major brain receptor ErbB4 (see below) are embedded in a pathway of $\mathrm{SZ}$ risk factors also comprising PI3K-AKT1. Dysregulated expression of such signaling modules could trigger abnormal activity in the signaling pathways they are embedded in and thereby ultimately lead to morphological and physiological dysfunctions with relevance for neuropsychiatric disorders, including SZ.

Although none of these genes alone is significant in GWAS studies, there is evidence for the involvement of all four genes in SZ (Emamian et al., 2004; Harrison and Law, 2006; Norton et al., 2006a; Nicodemus et al., 2010; Law et al., 2012; Hatzimanolis et al., 2013).

\subsection{Neuregulin1 isoforms}

The NRG1 gene belongs to a gene family, which includes six members (NRG1, NRG2, NRG3, NRG4, NRG5 and NRG6), with NRG1 being the best characterized (Falls, 2003; Mei and Nave, 2014). NRG1 is one of the largest mammalian genes ( 1.4 megabases in humans), located on the short arm of chromosome 8. Due to alternative splicing and differential promoter usage, the NRG1 gene gives rise to a 
family of growth and differentiation factors with more than 30 different isoforms (see Fig. 3 and Steinthorsdottir et al., 2004). NRG1 isoforms include secreted and transmembrane proteins, all of which share a common epidermal growth factor (EGF)-like signaling domain that is necessary and sufficient for the activation of receptor-tyrosine kinases of the ErbB family (Falls, 2003). The EGF-like domain exist in an $\alpha$-, and $\beta$-form, with the binding affinity of the $\beta$-form being 100 -fold stronger than the $\alpha$-form (Jones et al., 1999). NRG1 isoforms can be grouped into six main classes (types I - VI; see Figure 3 and Mei and Xiong, 2008) based on distinct Nterminal domains. Types IV to VI have not been extensively characterized and will not be further discussed in this thesis.

A

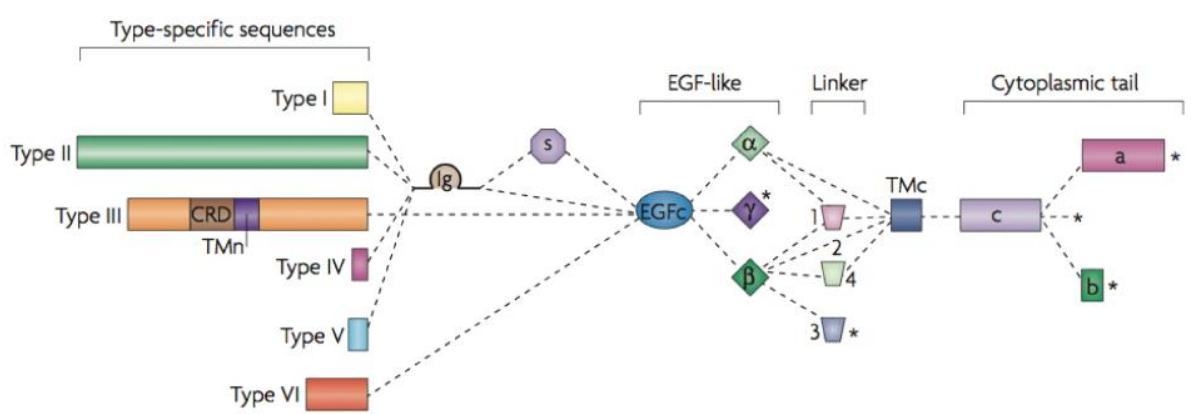

B

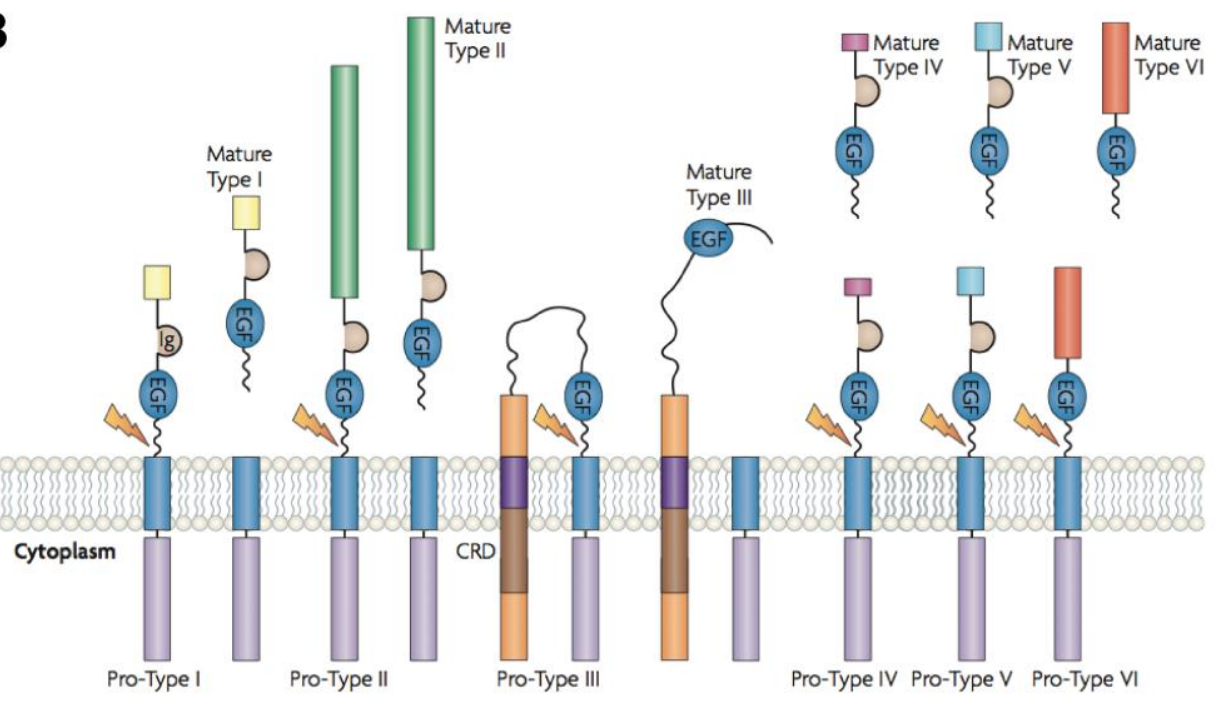

Fig. 3: NRG1 isoforms. (A) Alternative splicing and differential promoter usage give rise to at least $30 \mathrm{NRG} 1$ isoforms that can be grouped into six classes based on $\mathrm{N}$-terminal protein domains. All NRG1 isoforms share a common EGF-like domain. Ig-NRGs (Type I, II, IV and V) carry an immunoglobulin (Ig)-like domain. The N-terminal domain of NRG1 type III (cystein-rich domain; CRD) habors a second transmembrane domain (TMn). (B) Most NRG1 isoforms are produced as transmembrane proteins, also referred to as pro-NRG1s, which undergo proteolytic cleavage in the juxtamembrane linker (,stalk') region (flash) by proteases such as BACE1 or ADAMs metalloproteases, generating soluble („mature“) NRG1s. In case of NRG1 type III, the processed form remains membrane thethered due to the second TMn (modified from Mei and Xiong, 2008). 
NRG1 type I, II, IV and V isoforms contain an immunoglobulin (Ig)-like domain between the $\mathrm{N}$-terminal sequence and the EGF-like domain, and are therefore referred to as "Ig-NRGs" (see Fig. 3 and Harrison and Law, 2006; Mei and Xiong, 2008). Members of the type I group were identified independently and originally named heregulin (Holmes et al., 1992), acetylcholine receptor-inducing activity (ARIA) (Falls, 2003), or neu-differentiation factor (NDF) (Wen et al., 1992). The type II isoforms, also referred to as glial growth factor (GGF) (Marchionni et al., 1993), harbor a "kringle" domain, located N-terminally to the Ig-like domain. Sensory and motor neuron derived factor (SMDF) (Ho et al., 1995), nowadays referred to as NRG1 type III, harbors a cystein-rich domain (CRD) that serves as a second transmembrane domain (Fig. 3). These isoforms are also known as "CRD-NRGs" (Falls, 2003). Additional NRG1 variants are generated by splicing different linkers into the juxtamembrane 'stalk' region. Finally, the cytoplasmic tail of NRG1 is structurally variable, as isoforms of different length (a, b, c) exist (Wen et al., 1994) (Fig. 3).

Further complexity is added to NRG1 signaling by posttranslational modifications, e.g. glycosylation (Burgess et al., 1995), and proteolytic processing (Fig. 3B). NRG1 is cleaved in the stalk region by proteases such as BACE1 ( $\beta$-site APP-cleaving enzyme) (Hu et al., 2006, 2008; Willem et al., 2006) and metalloproteases of the ADAMs family, ADAMS17 (or tumor-necrosis factor- $\alpha$-converting enzyme; TACE), and ADAMS19 (or Meltrin beta) (Horiuchi et al., 2005; Yokozeki et al., 2007). While processing in the stalk region releases extracellular parts of NRG1 type I and type II proproteins from the cell surface for paracrine signaling, the $\mathrm{N}$-terminal fragment of the type III isoforms, is thought to remain membrane anchored (Fig. 3B). Their signaling is thought to proceed in a juxtacrine fashion (Mei and Xiong, 2008). However, it has recently been shown that further processing of type III isoforms releases the EGF-like domain, allowing paracrine signaling (Birchmeier and Nave, 2008; Willem et al., 2009; Fleck et al., 2013).

In addition to EGF-like domain-mediated ,forward signaling' to ErbB4, the intracellular C-terminal domain of NRG1 (NRG1-ICD or -CTF) has also been linked to signaling processes (Fig. 6). Specifically, it has been shown that after initial extracellular cleavage, $\gamma$-secretase can cleave the C-terminal domain, releasing the NRG1-ICD (Mei and Xiong, 2008; Talmage, 2008). NRG1-ICD signaling is of relevance for neuropsychiatric diseases, as a schizophrenia at risk mutation (substitution at valine 321) results in a NRG1 type III protein that fails to undergo $\gamma$-secretase-mediated cleavage and nuclear localization. Pharmacological and genetic studies have shown 
that this mutation impairs dendritic development independent of ErbB kinase activity (Chen et al., 2010).

Finally, NRG1 isoform expression has been shown to be differentially regulated during development. The most prominant isoforms in the human and rat brain are type III and type II isoforms, followed by type I and type V. It is important to note that all isoforms show dynamic expression profiles during developmental stages with expression peaks during early embryonic stages (embryonic day (E) 13) and around postnatal day $(P) 5$, suggesting functions in early neural development and the neonatal critical period. Most of the NRG1 isoforms are expressed by excitatory neurons, but are also present in GABAergic interneurons and astrocytes (Liu et al., 2011). NRG1 expression is also regulated by neuronal activity. Using a rat model of epileptic seizures induced by kainic acid (KA) treatment, type I and II isoforms were found to be significantly upregulated, while KA treatment had no effect on the expression of types III, V and VI isoforms. (Liu et al., 2011), suggesting isoformspecific regulation of distinct NRG1 isoforms.

In 2002, NRG1 was identified as a SZ susceptibility gene by fine mapping of the locus on chromosome $8 \mathrm{p} 22$ and haplotype association analysis performed in families of Icelandic origin. A "core at-risk" region in the 5' region of the NRG1 gene was identified and named the "Icelandic haplotype" by Stefansson et al. (2002). This region surrounds an exon that encodes the NRG1 type II-specific domain. Several but not all subsequent studies in other populations have replicated the association to $\mathrm{SZ}$, hence NRG1 has been on and off the lists of major SZ risk genes since then (Harrison and Weinberger, 2005). However, of the 23.094 single nucleotide polymorphisms (SNPs) found in the human NRG1 gene approximately $40 \%$ have been linked to SZ. ErbB4, the most prominent NRG1 receptor in the brain, has as well been identified as a SZ at risk gene in several studies (Nicodemus et al., 2006; Silberberg et al., 2006; Law et al., 2007). Both genes are on the list of major SZ susceptibility genes (for both NRG1 and ErbB4 see Schizophrenia Gene Resource, Vanderbilt University; http://bioinfo.mc.vanderbilt.edu/SZGR).

\subsection{The ErbB receptor family}

NRG1 signals via its EGF-like domain to receptor tyrosine kinases of the ErbB family (Mei and Xiong, 2008). The family name derives from the erythroblastic leukemia viral oncogene (v-erbB). ErbB receptors play an important role in the development of the heart and in different types of cancer. In the nervous system, ErbB receptors have been implicated in the regulation of a variety of neuronal developmental 
processes, including neuronal migration, Schwann cell- and oligodendrocytemediated myelination, axon guidance, synapse and neuromuscular junction formation (Mei and Xiong, 2008).

ErbB receptors are structurally related to the epidermal growth factor receptor (EGFR, HER or ErbB1), which was identified in the early 1980s. Since then the ErbB family has grown to four members, ErbB1 to 4, which together integrate at least 11 different ligands (Yarden and Sliwkowski, 2001). ErbB transmembrane receptors have similar structures, with a large glycosylated extracellular $\mathrm{N}$-terminal domain that carries the ligand binding site (seperated into four subdomains) (Fig. 4, 5). The cystein-rich domains II and IV can form 25 disulfide-bridges and play an important role in receptor dimerization (Fig. 4). All receptors have a transmembrane domain and a cytoplasmic tyrosine kinase domain with several potential tyrosine phosphorylation sites (Linggi and Carpenter, 2006). Upon ligand binding conformational changes are induced that render a dimerization arm accessible and allow the receptors to form homo- and heterodimers (Burgess et al., 2003), followed by activation of the intracellular tyrosine kinase domain. Dimerized receptors then auto- and transphosphorylate themselves at the C-terminus (Fig. 4). This process was shown to depend on receptor endocytosis (Gu et al., 2005; Yang et al., 2005; Liu et al., 2007). The intracellular phosphorylated tyrosine-residues then serve as binding sites for adaptor proteins that stimulate downstream signaling cascades.

ErbB receptors differ in their function. ErbB1 does not to bind NRG1, but is activated by at least seven other ligands, including EGF, TGF- $\alpha$, and heparin-binding EGF-like growth factor (HB-EGF). Also ErbB2 (also known as Her2 or Neu) does not bind NRG1, as it has an impaired ligand binding domain and functions only as a coreceptor (see Fig. 4 and Klapper et al., 1999). Although ErbB2 can not bind ligands, its conformation allows dimerization without ligand binding. ErbB2 is the preferred dimerization partner for other ErbBs, as heterodimers involving ErbB2 have a higher ligand affinity (Citri et al., 2003). In addition, interaction with ErbB2 allows its dimerization partners to bind additional ligands (Citri et al., 2003).

ErbB3 (also known as Her3) binds NRG1 and NRG2. Since ErbB3 is lacking a functional kinase domain, it must form heterodimers with other ErbB receptors in order to convey signals (see Fig. 4 and Guy et al., 1994). Thus, ErbB4, which binds to all NRGs, epiregulin, betacellulin and HB-EGF, is the only receptor that can form functional NRG-binding homodimers (Fig. 5). In addition, it forms heterodimers with all other ErbBs (Mei and Xiong, 2008). Although ErbB2 and ErbB3 alone are inactive, in vitro studies have shown that they form the most potent heterodimer to regulate cell growth and transformation (Wallasch et al., 1995; Pinkas-Kramarski et al., 1996). 
In summary the following functional ErbB receptor dimers can be activated by NRG1 binding: ErbB1-3, 1-4, 2-3, 2-4, 3-4, 4-4 (Mei and Xiong, 2008).

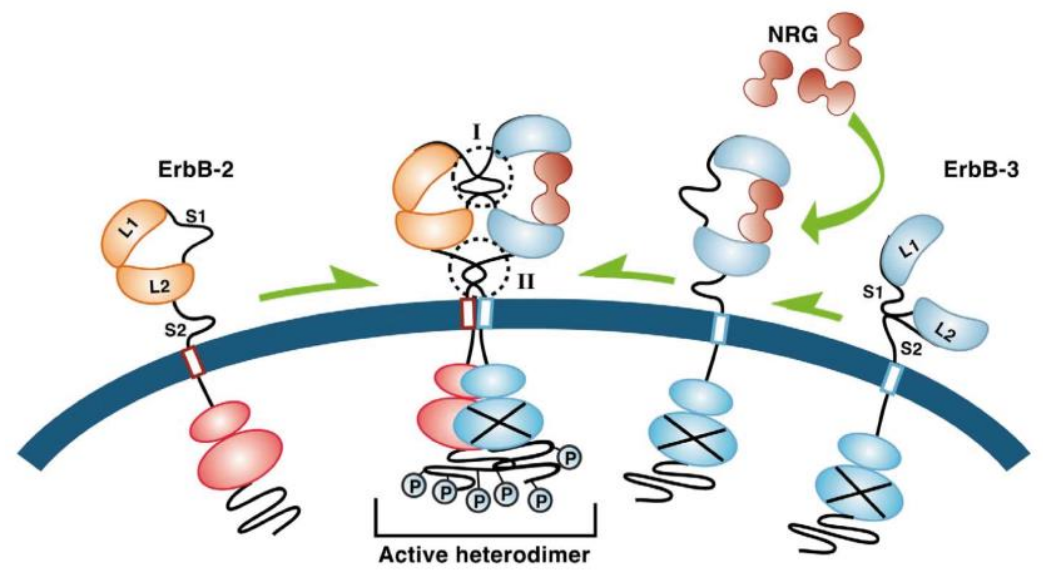

Fig. 4: ErbB receptors: ligand binding and receptor dimerization. Binding of NRG molecules to ErbB receptors is illustrated for an ErbB2/ErbB3 heterodimer. ErbB2 has an inactive ligand-binding site and only functions as a co-receptor. ErbB3 is lacking an active intracellular kinase domain and also only functions as a heterodimer. Conformational changes are induced by ligand binging and result in receptor dimerization, which leads to auto- and transphosphorylation of intracellular tyrosin residues and recruitment of adaptor proteins, and activation of downstream signaling cascades (modified from Citri et al., 2003).

Activation of ErbB receptors and recruitment of adaptor proteins triggers intracellular signaling pathways that regulate cellular responses, including stimulation or inhibition of proliferation, apoptosis, migration, differentiation, and adhesion (Yarden and Sliwkowski, 2001).

\subsection{The ErbB4 receptor}

Analyses of ErbB receptor expression patterns and functions revealed that ErbB4 is the main neuronal receptor for NRG1 in the brain (Fig. 5). ErbB4 is predominantly expressed by the majority of $\mathrm{PV}^{+}$interneurons (Neddens and Buonanno, 2009, 2011; Vullhorst et al., 2009; Neddens et al., 2011). Similar to the multimodal signaling properties of NRG1, ErbB receptors exhibit different signaling properties. In canonical forward signaling, the aforementioned auto- and transphosphorylation and recruitment of adaptor proteins triggers intracellular signaling pathways, including the Raf-MEK-ERK and PI3K-AKT-S6K pathways (Si et al., 1999; Fu et al., 2001; Yarden and Sliwkowski, 2001; Bjarnadottir et al., 2007).

Similar to NRG1, ErbB4 transcripts are alternatively spliced, resulting in four different isoforms by differential pairing of the cytoplasmic C-terminal CYT-1 and CYT-2 domains and the extracellular (,juxtamembrane") JMa and JMb domains. The four 
ErbB4 isoforms show distinct tissue- and brain-region-specific expression patterns and trigger distinct signaling cascades. Both cytoplasmic isoforms regulate DNA synthesis and proliferation by activation of the Shc-Ras-MAPK signaling pathway. However, only the CYT- 1, but not CYT- 2 isoform, can activate PI3K and subsequently AKT (see Fig. 5 and Elenius et al., 1999), that regulates chemotaxis and apoptosis (Elenius et al., 1997; Sawyer et al., 1998; Junttila et al., 2000; Kainulainen et al., 2000; Rio et al., 2000; Lewis et al., 2005; Norton et al., 2006b). Both JMa and JMb can bind NRG1, but only JMa is a substrate for TACE-mediated cleavage and release of an extracellular domain of ErbB4 (ecto-ErbB4, Fig. 5) (Sawyer et al., 1998; Rio et al., 2000; Cheng et al., 2003; Arasada and Carpenter, 2005). TACE-mediated cleavage is part of non-canonical ErbB4 forward signaling (Fig. 6) and mediated by ligand-binging or protein kinase C (PKC) activity (Vecchi et al., 1996; Zhou and Carpenter, 2000; Zhu et al., 2000). This first cleavage renders the remaining protein fragment accessible to $\gamma$-secretase-mediated cleavage and release of the ErbB4 intracellular domain (ErbB4-ICD), similar to NRG1-ICD cleavage (Fig. 6). The ErbB4-ICD can be translocated into the nucleus and is thought to act as a chaperon that promotes the nuclear transport of transcription factors STAT5 (signal transducer and activators of transcription) and YAP1 (Yes-associated protein-1), thereby regulating gene expression (Ni et al., 2001; Lee et al., 2002; Komuro et al., 2003; Omerovic et al., 2004; Williams et al., 2004; Sardi et al., 2006; Mei and Xiong, 2008). 
A

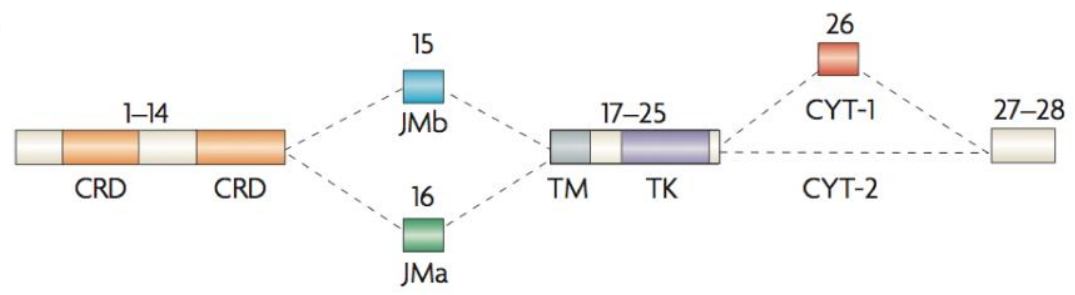

B

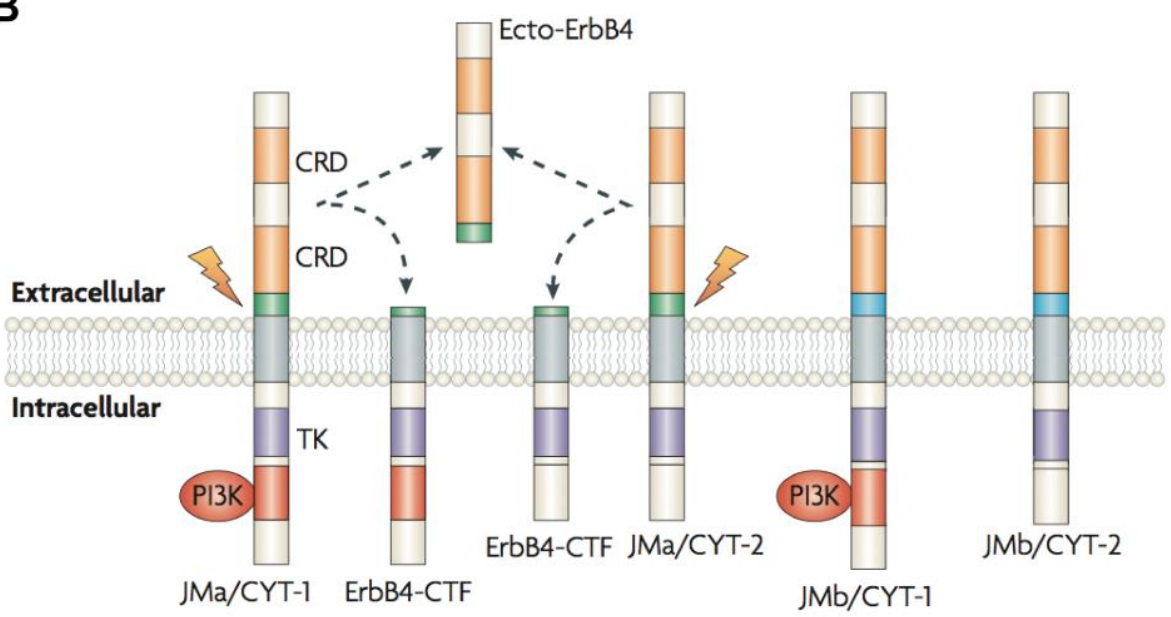

Fig. 5: Splice isoforms of the ErbB4 receptor. (A) Four different ErbB4 variants are produced by alternative splicing of juxtamembrane regions $\mathrm{JMa}$ or $\mathrm{JMb}$ and the cytoplasmic CYT-1 or CYT-2 domains. (B) ErbB4 is produced as a singlepass membrane protein with two extracellular cysteine-rich (CRD) domains, important for receptor dimerization. Only the JMa region is a substrate of TACE-mediated cleavage (flash) and release of the extracellular domain of ErbB4 (ecto-ErbB4). Both cytoplasmic isoforms can activate the Shc-Ras-MAPK signaling pathway, but only the CYT- 1 containing variant activates PI3K and AKT signaling (modified from Mei and Xiong, 2008).

ErbB4, as the only member of the ErbB family, contains a C-terminal T-V-V peptide motive that allows the interaction with PDZ-domains of other proteins (Garcia et al., 2000). PDZ were originally identified in PSD95 (Cho et al., 1992; Kistner et al., 1993) and a colocalization of ErbB4 and PSD95 was observed in the excitatory postsynapse (Garcia et al., 2000; Huang et al., 2000; Ma et al., 2003). It has been suggested that ErbB4/PSD95 signaling could potentially modulate NMDA and AMPA receptor functions and $\mathrm{K}^{+}$channels (Kim and Sheng, 2004). Thus, interactions of ErbB4 with synaptic proteins, like the scaffold protein PSD95, could link NRG1/ErbB4 signaling to synaptic organization by impacting on synaptic integrity and function.

\subsection{NRG1/ErbB4 signaling in cortical networks during health and disease}

NRG1 and ErbB4 are implicated in regulating various aspects of neural development, including proliferation, migration and differentiation. Dysfunctions in these processes 
have been considered relevant for neuropsychiatric disorders, including SZ, as reduced numbers of inhibitory interneurons (Benes et al., 1991; Chance et al., 2005; Holt et al., 2005; Levitt, 2005; Heckers and Konradi, 2014), as well as reduced expression of GAD67 in $\mathrm{PV}^{+}$interneurons of the dorsolateral prefrontal cortex and disturbed inhibitory functions (Benes et al., 1991; Akbarian et al., 1995; Hashimoto et al., 2003; Lewis et al., 2005; Farzan et al., 2010; Ongür et al., 2010; Yoon et al., 2010) have been described in cases of SZ. In addition, alterations in dendritic spines (Penzes et al., 2011) and deficits in cortical synchronization were observed in SZ (Uhlhaas and Singer, 2010).

Most of the NRG1 at-risk haplotypes have been mapped to the non-coding region of the NRG1 gene (Stefansson et al., 2002; Weickert et al., 2012), suggesting that rather than altered protein functions per se, changes in NRG1 expression levels could cause abnormal NRG1 functions in SZ. Indeed reduced and increased NRG1 expression were found in postmortem studies of SZ patients (Law et al., 2006; Bertram et al., 2007), including elevated expression of CRD-NRG1, the predominant isoform of NRG1 in the human brain (Liu et al., 2011; Weickert et al., 2012). In addition, NRG1-induced ErbB4 hyperphosphorylation was observed in postmortem brains of SZ patients supporting this idea (Hahn et al., 2006).

By in situ hybridization and immunostainings, NRG1 expression has been detected in pyramidal neurons of the human and rat cortex and hippocampus, for instance in hippocampal CA3 pyramidal neurons, that form the Schaffer collaterals by projection to CA1 neurons (see Fig. 7 and Law et al., 2004; Okada and Corfas, 2004). ErbB4 on the other hand is expressed by GABAergic interneurons (see Fig. 7 and Neddens and Buonanno, 2009, 2011; Vullhorst et al., 2009; Neddens et al., 2011) and has been colocalized with PSD95 in the excitatory postsynapse (Garcia et al., 2000; Huang et al., 2000). Interaction of ErbB4 with PSD95 has been shown to increase the activation of the receptor by NRG and subsequent MAP-kinase signaling (Huang et al., 2000). The postulated localization of NRG1 and ErbB4 suggests a potential involvement in the regulation of neuronal migration, synaptic plasticity and pyramidal neuron-interneuron interaction (Fig. 6, 7).

Modelling loss- and gain-of-functions of NRG1 and ErbB4 in cell culture and transgenic mouse models has become a valuable tool to study their functions in vitro and in vivo. These approaches have delivered important insights into NRG1/ErbB4 signaling functions during the formation and maintenance of cortical circuitry under normal and disease conditions.

Due to a heart defect, ErbB4 null mutant mice die at E10.5 (Gassmann et al., 1995), only when ErbB4 is expressed under a heart-specific myosin-promoter (,heart 
rescue“, ErbB4 ${ }^{-/} H E R 4^{\text {heart }}$ ) the null mutant is viable (Tidcombe et al., 2003). Such genetic ablation of ErbB4 in mice was shown to impair GABAergic interneuron migration from the subpallium to the cortex (Flames et al., 2004), and thalamocortical axonal pathfinding (López-Bendito et al., 2006). Interneurons ultimately fail to reach the cortex, leading to reduced numbers of GABAergic interneurons in ErbB4 ${ }^{--}$ mice (Flames et al., 2004; Neddens and Buonanno, 2009). In line with this, ErbB4 ${ }^{-1-}$ mutant mice have comprised inhibitory circuits (Neddens and Buonanno, 2009; Fazzari et al., 2010; Wen et al., 2010; Del Pino et al., 2013).

Treatment of hippocampal slices with soluble NRG1 was shown to block LTP (Huang et al., 2000; Kwon et al., 2005; Bjarnadottir et al., 2007). Concordantly, acute treatment of hippocampal slices from ErbB4 ${ }^{-/}$mice with soluble NRG1 had no effect on LTP (Pitcher et al., 2008). Conversely, acute blocking of ErbB kinase activity in hippocampal slices prevented this blockade, and ablation of ErbB4 in ErbB4 ${ }^{-/}$mice was shown to result in enhanced LTP at the Schaffer-collateral CA1 synapse (Pitcher et al., 2008; Chen et al., 2010; Shamir et al., 2012). In addition, loss of ErbB4 also lead to enhanced limbic epileptogenesis, an effect reversed by soluble NRG1 incubation ( $\mathrm{Li}$ et al., 2011; Tan et al., 2011), demonstrating an important role of NRG1/ErbB4 signaling in balancing inhibitory circuit activity.

Further, $\mathrm{ErbB}^{-/}$and $\mathrm{PV}^{+}$interneuron-restricted $\mathrm{PV}-\mathrm{Cre}{ }^{\star} \mathrm{ErbB} 4^{-/}$mice were shown to exhibit novelty-induced hyperactivity and deficits in prepulse inhibition (PPI), in line with findings in SZ patients. Interestingly, only ErbB4 ${ }^{-/}$mice, but not $P V-C r e{ }^{*} E r b B 4^{-/}$ mutants, showed reduced anxiety-like behavior and impairments of contextual and cued fear memory, in line with broad ErbB4 expression in $\mathrm{PV}^{-}$cells of the amygdala (Shamir et al., 2012; Yin et al., 2013b). Further, combined ablation of ErbB2 and ErbB4 in the CNS of mice impaired NMDA receptor/PSD95 clusters and dendritic spine maturation, increased aggressive behavior and reduced PPI (Barros et al., 2009).

Many in vitro and slice culture studies on NRG1/ErbB4 signaling have used the recombinant EGF-like signaling domain (commonly referred to as "soluble NRG1"). These studies have for instance shown that treatment of cultured neurons or brain slices with soluble NRG1 modulates glutamatergic, GABAergic cholinergic and dopaminergic neurotransmission (Gu et al., 2005; Kwon et al., 2005; Woo et al., 2007; Ting et al., 2011). For instance, treatment of cultured prefrontal cortex pyramidal neurons with the recombinant EGF-like domain was shown to reduce NMDA receptor-mediated excitatory postsynaptic currents (EPSC) (Gu et al., 2005), and treatment of hippocampal slices resulted in reduced AMPA receptor-mediated EPSCs and triggered the internalization of surface glutamate receptor 1-containing 
AMPA receptors (Kwon et al., 2005). Soluble NRG1 also promoted dendritic spine maturation (Barros et al., 2009).

In line with this, Thy1.2-mediated overexpression of CRD-NRG1 in mice (NRG1-tg) reduced LTP and altered dendritic spine morphology. Conditional inactivation of NRG1 from projection neurons, using CamKII-Cre driver mice, had a similar effect on LTP, suggesting an inverted U-shaped model (Agarwal et al., 2014). In line with this, a disturbed excitatory/inhibitory (E/I) balance was found CA1 pyramidal neurons in both NRG1-tg and CamKII-Cre and Emx1-Cre*Nrg 1//f mutant mice. NRG1 overexpression lead to increased IPSC frequencies, but not amplitudes, suggesting that NRG1 overexpression shifts the E/I synaptic balance towards enhanced inhibition, probably by increased GABAergic input (Agarwal et al., 2014). Alterations in the $E / I$ balance were suggested to result in deficits in cortical synchronization as implicated in SZ (Uhlhaas and Singer, 2010). However, it is important to note that studies with isoform-specific mouse mutants revealed that the structural variability of NRG1 isoforms and their different expression patterns translate into differential functions (Meyer et al., 1997; Wolpowitz et al., 2000). Thus, only overexpression of CRD-NRG1 impaired LTP, whereas overexpression of Ig-NRG1 impaired $\gamma$ oscillations, but not LTP (Deakin et al., 2012). In line with isoform-specific functions of NRG1 variants, the migration of GABAergic interneurons from the subpallium to the developing cortex is regulated by an interplay of long- and short-range attractive signals of distinct NRG1 isoforms and ErbB4 receptors. CRD-NRG1 isoforms form a permissive corridor along which interneurons migrate, while Ig-NRGs form a diffusible signal attracting them toward the cortex (Flames et al., 2004).

Alterations in dendritic spines have been observed in several SZ cases (Penzes et al., 2011). NRG1/ErbB4 signaling has been linked to synaptogenesis, neurite outgrowth and dendritic spine dynamics. Mice lacking BACE1 displayed reduced numbers of dendritic spines in hippocampal neurons and exhibit SZ-relevant phenotypes, including reduced PPI and novelty-induced hyperactivity (Savonenko et al., 2008), suggesting that proteolytic processing of NRG1 is important for its signaling functions in cortical networks. In a recent study, the NRG1-ICD and $\gamma$ secretase subunit APH1B were linked to dendritic spine formation (Fazzari et al., 2014), indicating that CRD-NRG1 backsignaling might play an important role in the regulation of synaptogenesis and synaptic plasticity in vivo. A SZ at-risk mutation in $N R G 1$, a valine-to-leucine substitution at valine 321 prevents $\gamma$-secretase-mediated NRG1 cleavage, subsequent nuclear localization, and transcriptional activation. Further, this mutation was shown to impair dendritic development independent of 
ErbB receptor kinase activity (Chen et al., 2010), underlining the cell autonomous backsignaling properties of CRD-NRG1 and their relevance for SZ (Fig. 6).

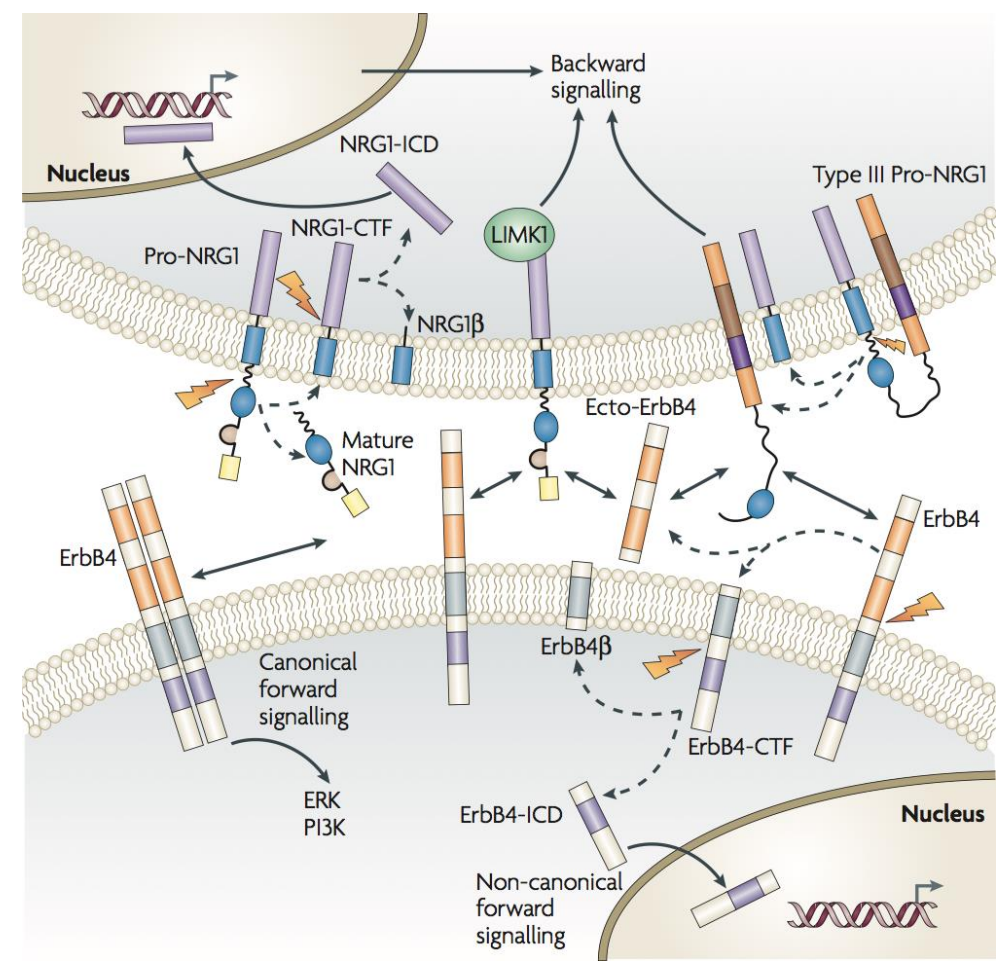

Fig. 6: Model of canonical and non-canonical NRG1/ErbB4 signaling. Both NRG1 and ErbB4 can serve as ligand and receptor. Canonical forward signaling includes cleavage (flash) of NRG1 in the stalk-region, followed by paracrine or juxtacrine signaling to ErbB4, which triggers downstream signaling cascades (ERK, PI3K). $\gamma$-secretase mediated ErbB4 cleavage induces ErbB4-CTF-mediated non-canonical forward signaling, including nuclear translocation and altered gene expression. TACE-mediated extracellular cleavage of ErbB4 produces ecto-ErbB4, which can signal to NRG1, possibly inducing $\gamma$-secretase-mediated NRG1 cleavage, nuclear translocation of the NRG1-ICD and altered gene expression. Interaction of the NRG1-ICD with LIMK1 may also trigger backward signaling possibly involved in actin cytoskeleton remodelling and dendritic spine dynamics (modified from Mei and Xiong, 2008).

Apart from its functions in transcriptional regulation, interaction of the NRG1-ICD with LIM Kinase 1 (LIMK1) was reported (see Fig. 6 and Wang et al., 1998). LIMK1 is a LIM and PDZ domain containing kinase, which has been implicated in the modulation of dendritic spines by regulating actin cytoskeleton dynamics. Abnormalities in spine morphology and synaptic functions have been reported for $L I M K 1^{-1-}$ mice (Meng et al., 2002, 2003). LIMK1 and its family member LIMK2 are key regulators of the actin depolymerizing factor cofilin. LIMK1 itself is regulated via phosphorylation by Rho GTPase-associated protein kinases PAK and ROCK (Edwards et al., 1999; Maekawa et al., 1999), and thus links Rho GTPases Rac, Rho and Cdc42 (Arber et al., 1998; Yang et al., 1998) to actin reorganization. Many of these interaction partners have also been implicated as downstream targets of ErbB signaling, thus 
strengthening the importance of LIMK1 interactions with NRG1/ErbB4 signaling in the context of dendritic spine remodelling and synaptic plasticity. This is supported by a recent study in which it was shown that overexpression of the NRG1 type I isoform in transgenic mice alters LIMK1 expression and cofilin phosphorylation. Interestingly, these findings were partly independent from ErbB4 (Yin et al., 2013a).

Taken together the NRG1/ErbB4 signaling module plays a critical role in cortical development and synaptic functions in the mature brain. It regulates various aspects of neuronal plasticity and connectivity and has been implicated in multiple processes that could underlay the pathomechanisms of neuropsychiatric disorders, including SZ. As most of the NRG1 at-risk haplotypes have been mapped in the non-coding region of the NRG1 gene, chronic changes in NRG1/ErbB4 signaling could confer increased risk for SZ. However, the precise pathomechanism underlying SZ pathogenesis still remain elusive.

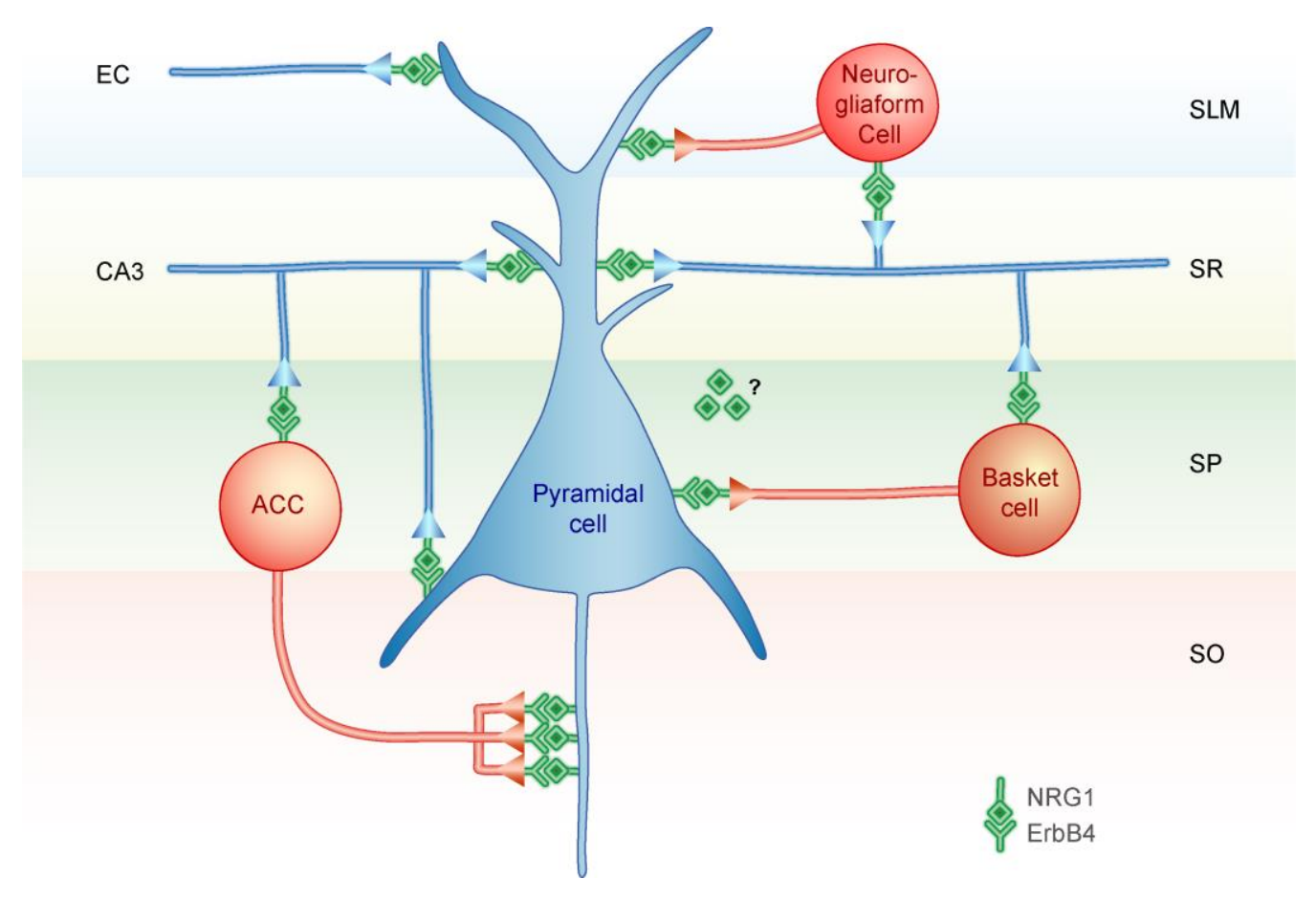

Fig. 7: NRG1/ErbB4 signaling regulates various aspects of cortical networks. Simplified hippocampal microcircuit and possible sites of NRG1/ErbB4 signaling. NRG1 is mainly expressed by pyramidal neurons, whereas ErbB4 is predominantly expressed in subsets of GABAergic interneurons, including basket cells and chandelier synapse-forming axoaxonic interneurons (ACC), and neurogliaform cells. According to this model, NRG1 and ErbB4 in different cell types and subcellular locations serve distinct regulatory functions at cortical synapses. Question mark indicates the unclear role of soluble NRG1 in paracrine signaling. CA3, Schaffer collateral-CA1 projections; EC, input from entorhinal cortex; SLM, Stratum lacunosum-moleculare; SO, Stratum oriens; SP, Stratum pyramidale; SR, Stratum radiatum. (kindly provided by Dr. Markus H. Schwab, adapted from Lewis et al., 2005). 


\subsection{Aim of this study}

$\mathrm{SZ}$ is considered as a disorder of brain disconnectivity. Human NRG1 and ERBB4 genes are possible genetic risk factors for SZ. NRG1/ErbB4 signaling regulates synaptic functions in the brain, but the underlying pathomechanisms linked to SZ remain elusive. Reduced and increased expression of NRG1 and ErbB4 hyperstimulation has been observed in post mortem brain of SZ patients. Thus, altered NRG1 expression and ErbB4 activation could represent a possible pathomechanism with relevance for SZ. To test this hypothesis, the first aim of the PhD project was to systematically investigate loss- and a gain-of-function mouse models of NRG1 for changes in brain functions, including development of cortical interneurons.

NRG1 isoforms show dynamic and complex expression patterns in the brain. Thus, cell type- or stage-specific changes in NRG1 expression could differentially affect brain functions. Therefore, the second aim of the $\mathrm{PhD}$ project was to characterize a novel conditional NRG1 transgenic mouse line, and to model cell type- and stagespecific overexpression of CRD-NRG1 in vivo. The third aim of this project was to examine consequences of stage-specific, cortical-restricted overexpression of CRDNRG1 on brain development and behavioral functions. Further, the project aimed at obtaining a first insight into molecular pathomechanisms of cortical network dysfunctions downstream of hyperstimulated NRG1/ErbB4 signaling with relevance for schizophrenia. 


\section{Results}

\subsection{Consequences of NRG1 deficiency on interneuronal migration and integration}

GABAergic interneurons control the excitatory-inhibitory balance in cortical networks and mediate the precise gating of information (Vogels and Abbott, 2009; Marin, 2012). Abnormal inhibitory functions have been reported in schizophrenia (SZ) patients (Lewis et al., 2005; Farzan et al., 2010; Ongür et al., 2010; Yoon et al., 2010). ErbB4, the main neuronal receptor for NRG1 in the brain, is expressed in GABAergic interneurons (Neddens and Buonanno, 2009, 2011; Vullhorst et al., 2009; Fazzari et al., 2010), and plays an important role for interneuronal development (Flames et al., 2004; Neddens and Buonanno, 2009). The human NRG1 gene is a major SZ susceptibility gene (Li et al., 2006; Ayalew et al., 2012). Virtually all of the "at-risk" haplotypes are located in non-coding regions of the gene (Stefansson et al., 2002; Weickert et al., 2012), suggesting that altered expression of NRG1 may represent a component of SZ pathology. Both, reduced and increased expression of NRG1 variants was found in post-mortem brains of SZ patients (Law et al., 2006; Bertram et al., 2007).

To investigate the effects of NRG1 deficiency on interneuronal migration and integration into the cortical network, we examined conditional NRG1 mutants. To delete NRG1 from all forebrain progenitor cells beginning at embryonic day (E) 10 (Fig. 8A) we crossbred floxed $\mathrm{Nrg} 1$ mice ( $\mathrm{Li}$ et al., 2002) to EMX1-Cre driver mice (Gorski et al., 2002, referred to as Emx-Cre mice). The cell population derived from the Emx1 cell lineage includes radial glia, Cajal-Retzius cells, glutamatergic neurons, astrocyes and oligodendrocytes, but not interneurons. The Emx-Cre recombination pattern is shown by X-Gal histochemistry on a brain section of an Emx-Cre*R26RfloxLacZ double-transgenic mouse at postnatal day (P) 46 (Fig. 8A). This approach lead to a $\sim 80 \%$ reduction of NRG1 protein in the forebrain of Emx-Cre ${ }^{*} \mathrm{Nrg} 1^{t / f}$ mice (Agarwal et al., 2014).

To determine the number of GABAergic interneurons in the postnatal brain of NRG1 deficient mice, coronal paraffin sections of Emx-Cre* $\mathrm{Nrg} 1^{\mathrm{ft} / f}$ mice and controls at P14 were stained for the interneuron marker GAD67 and interneuron numbers were quantified in the somatosensory cortex and hippocampus (bregma -1.7). Total interneuron numbers in the hippocampus of $\mathrm{Emx}$-Cre* $\mathrm{Nrg} 1^{t / f}$ mice were not altered compared to controls (Fig. 8D). In addition, the subcortical distribution of GAD67 ${ }^{+}$ interneurons showed no differences in Emx-Cre ${ }^{*} \mathrm{Nrg}^{t / f}$ mice compared to controls (Fig. 8E). Finally, cortical layer width (based on GAD67 immunoreactivity) was 
A

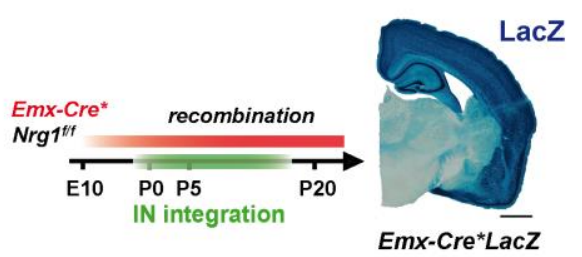

B

C
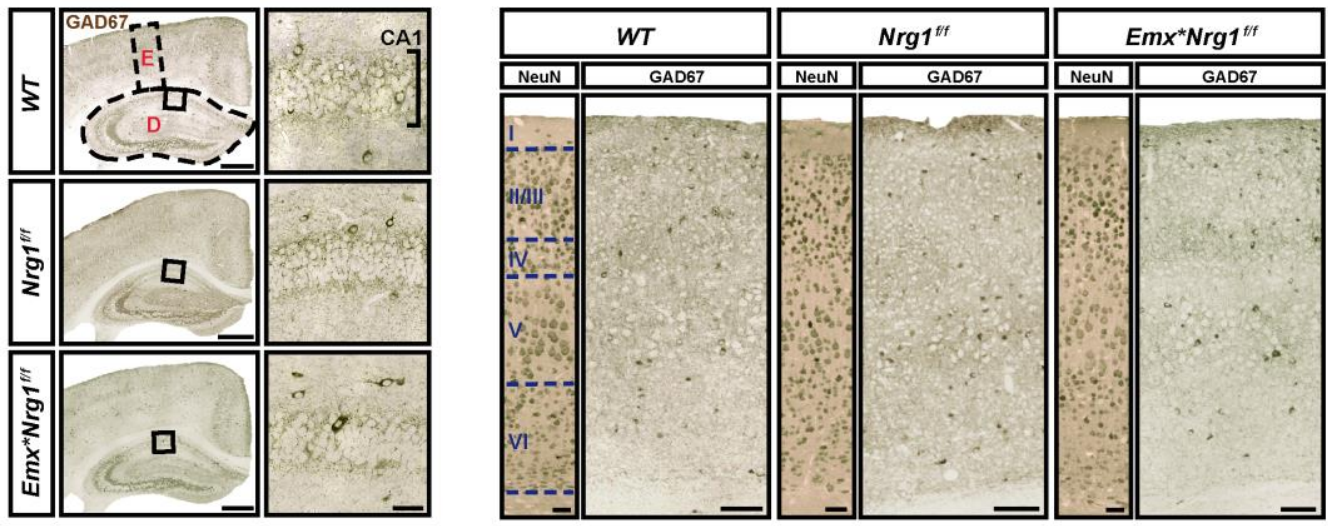

D

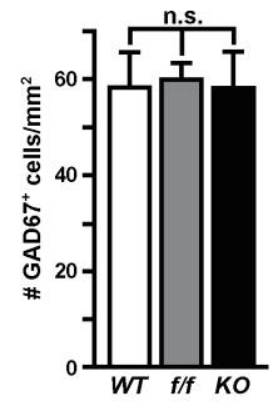

F

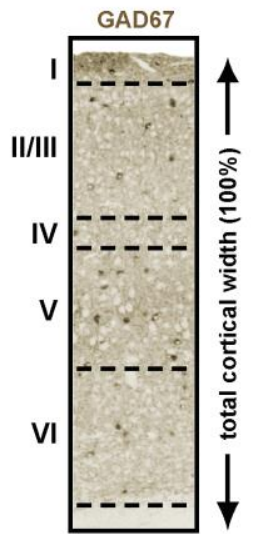

E
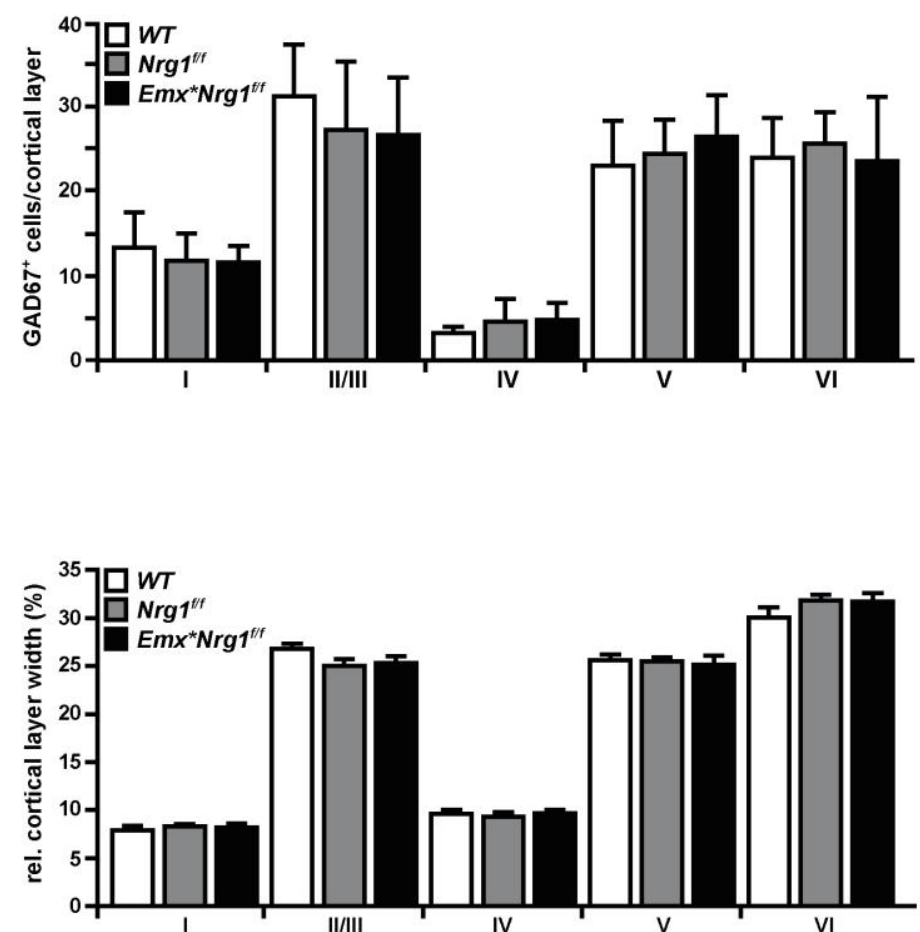

Fig. 8: Embryonic NRG1 signaling is dispensable for cortex lamination and interneuronal migration. (A) Using the Emx1-Cre driver line, NRG1 elimination can be achieved in embryonic stages, overlapping with network integration of cortical interneurons (IN). X-gal histochemistry on a $20 \mu \mathrm{m}$ coronal cryo-brain section from an $E m x-C r e^{*} R 26 R$ floxLacZ double-transgenic mouse (P46) shows Cre-mediated recombination of lacZ reporter in forebrain projection neurons and glial cells. Scale bar, $1 \mathrm{~mm}$. (B to $E)$ Normal numbers and cortical positions of GAD67 $7^{+}$interneurons in $E m x^{*} N r g 1^{t / f}$ mutants during cortical maturation. (B) Immunostaining for GAD67 on coronal paraffin brain sections from $E m x^{*} N r g 1^{f / f}$ mutants and controls ( $\mathrm{Nrg}^{t / f}$ and $W T$ ) at P14. Higher magnifications (right) show the hippocampal CA1 region (boxed in overviews). Scale bars, $500 \mathrm{~mm}$ and $50 \mathrm{~mm}$ (CA1 region). (C) Immunostaining for NeuN and GAD67 (higher magnification of boxed area E in B) reveals 
normal layering (I-VI, cortical layers) and interneuron positions in the somatosensory cortex of $E m x^{*} N r g 1^{t f f}$ mutants in comparison to controls (Nrg $1^{t / f}$ and $W T$. Bregma -1.7; scale bars, $50 \mathrm{~mm}$ (NeuN), $100 \mathrm{~mm}$ (GAD67). (D) Quantification of $\mathrm{GAD67}^{+}$interneurons in the hippocampus shows no difference in interneuron numbers at P14 (marked area D in B) of $E m x^{*} N r g 1^{t / f}$ mutants and controls $\left(N r g 1^{t / f}\right.$ and $\left.W T\right)$. $n=6 /$ genotype. (E) Quantification of GAD67 $7^{+}$interneurons per layer in the neocortex of $E m x^{*} N R G 1^{t / f}$ mutants and controls $\left(N r g 1^{t / f}\right.$ and $W T$ ) at P14 reveals no difference in interneuron numbers and layering (boxed area $E$ in B). $n=6 / g$ enotype. Error bars represent SEM. (F) Normal cortical lamination in Emx-Nrg $1^{t / f}$ mutants. Relative cortical layer width was determined based on GAD67 immunostainings on coronal paraffin brain sections (left panel, representative micrograph from $\mathrm{Nrg}^{1 / f}$ mice, bregma $-1.7 ; n=6$ per genotype; error bars represent SEM).

normal in Emx-Cre* Nrg1/f/f mice at P14 compared to controls (Fig. 8F). Similar findings were obtained at one year of age (data not shown).

Taken together, NRG1 signaling appeared to be dispensible for interneuron migration and their integration into cortical layers, and NRG1 mutants did not phenocopy the ErbB4 null mutation.

\subsection{Elevated CRD-NRG1 expression leads to sustained ErbB4 hyperstimulation, altered interneuron functions, and abnormal behavior}

In a complementary approach, we tested whether chronically increased NRG1 expression could affect interneuronal migration and neocortical network assembly. CRD-NRG1 is the most prominant NRG1 isoform in the brain (Liu et al., 2011) and elevated CRD-NRG1 expression has been reported in post-mortem tissue of SZ patients (Weickert et al., 2012). To test whether CRD-NRG1 serves as a signal for ErbB-receptor-mediated cortical network regulation, we examined $\mathrm{Nrg} 1$-tg mice that express CRD-NRG1 under control of the neuronal Thy1.2 promoter (Fig. 9A) (Michailov et al., 2004). In these mice transgene expression is initiated around E16 and CRD-NRG1 accumulates on the surface of projection neurons in the neocortex and hippocampus, but is absent from interneurons, astrocytes, and oligodendrocytes (Agarwal et al., 2014).

First, we tested whether chronic CRD-NRG1 overexpression alters ErbB4 receptor activation. A western blot analysis of hippocampus protein lysates from $\mathrm{Nrg} 1$-tg mice revealed CRD-NRG1 overexpression at 4 months of age. CRD-NRG1 was expressed as a full length protein $(\sim 140 \mathrm{kDa})$ and a processed form $(\sim 60 \mathrm{kDa}$; presumably the NRG1 CTF after stalk region cleavage) (Fig. 9B). Phosphorylation of ErbB4 was detected using an antibody directed against the phosphorylated tyrosin residue Tyr1284, a binding site for the adapter protein ,SH2 domains containing transforming protein 1' (SHC1) (Schulze et al., 2005). Western blot analysis revealed 
A

B
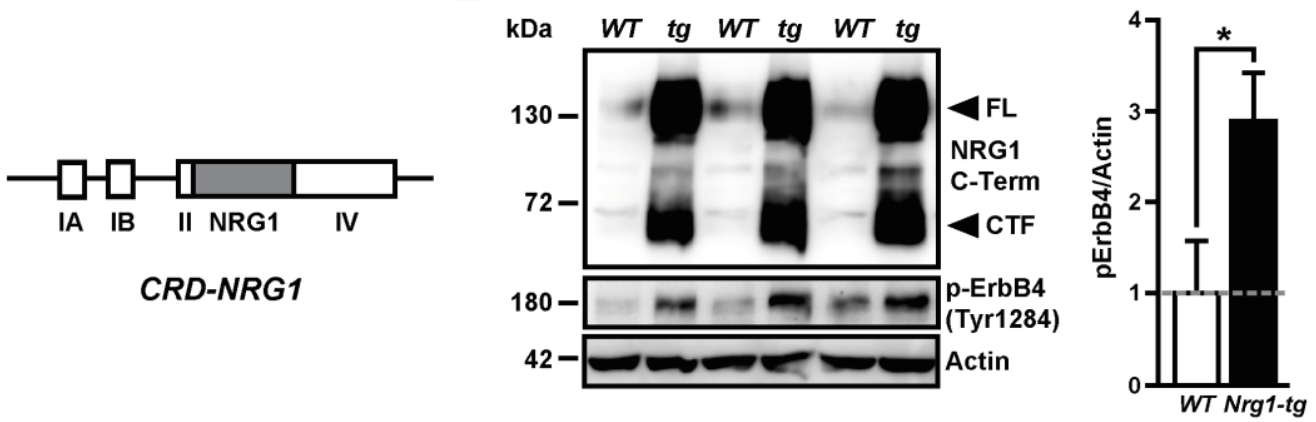

C
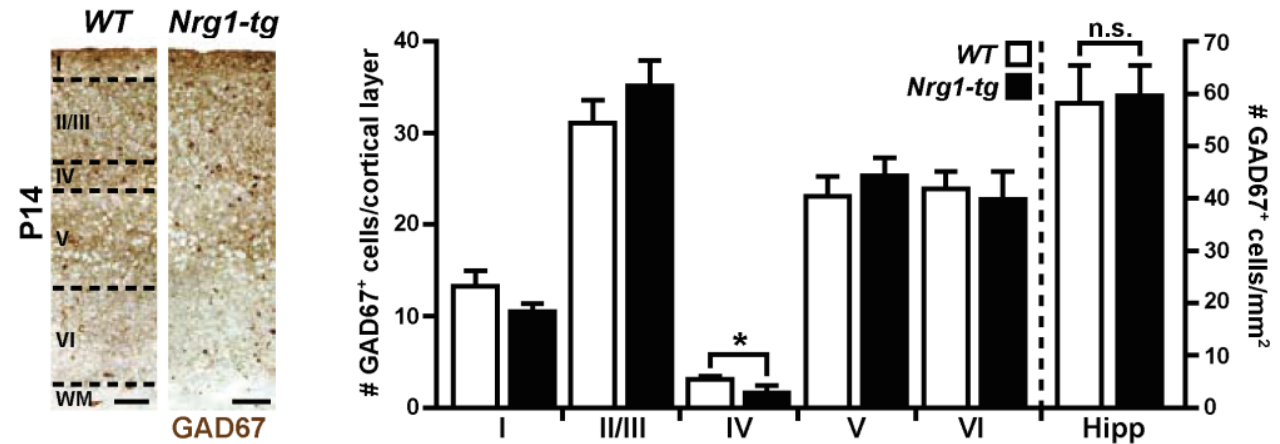

D
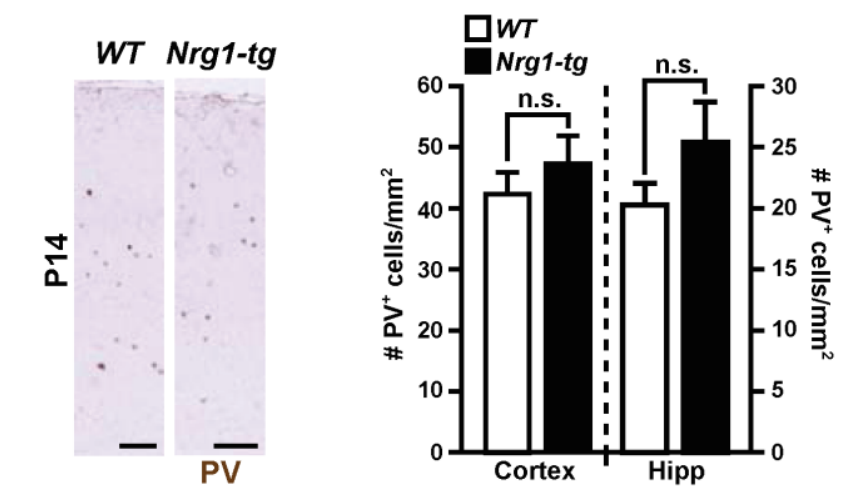

E
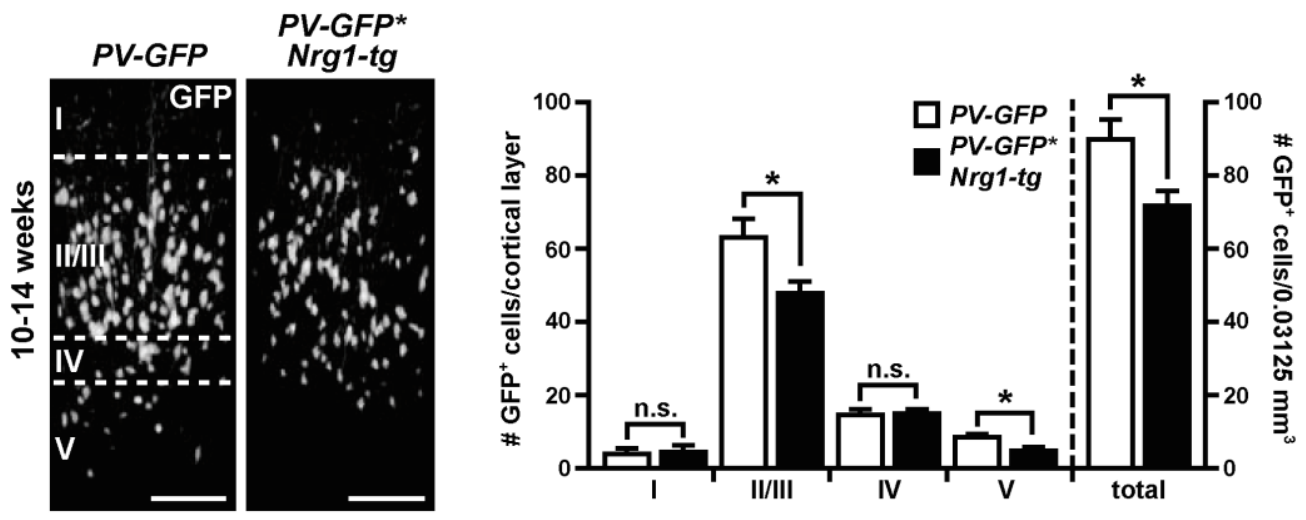

Fig. 9: Elevated CRD-NRG1 expression results in ErbB4 hyperphosphorylation and altered numbers of GABAergic interneurons. (A) Structure of the Thy1.2 transgene cassette used in Nrg1-tg mice (grey box, CRD-NRG1 CDNA; white boxes, exons I-IV of the Thy1.2 gene). (B) Overexpression of CRD-NRG1 results in a chronic ErbB4 hyperphosphorylation in the hippocampus of Nrg1-tg mice. Western blot analysis of hippocampal total protein sucrose lysates from Nrg1-tg mice (tg) and WT (4 months). 
Densitometric quantification of phosphorylated ErbB4 (Tyr1284) bands. Integrated density values were normalized to $\beta$-actin loading control $\left(n=3 /\right.$ genotype; ${ }^{*} p<0.05$; Mann-Whitney $U$ test). CTF, NRG1 C-terminal fragment; FL, full length; kDa, kilodalton. (C) (Left) Immunostaining for GAD67 on $5 \mu \mathrm{m}$ coronal paraffin sections from brains of $W T$ and $N r g 1$-tg mice at P14 (somatosensory cortex; bregma -1.7). Scale bars, $100 \mathrm{~mm}$. (Right) Quantification of GAD67 interneurons reveals a loss of interneurons in layer IV of the cortex $(n=$ 6/genotype; ${ }^{*} p<0.05$, Mann-Whitney U test). I-VI, cortical layers; WM, white matter. (D) (Left) Immunostaining for Parvalbumin (PV) as in (C). Scale bars, $100 \mathrm{~mm}$. (Right) Quantification of $\mathrm{PV}^{+}$interneurons across all cortical layers shows no difference in total cell numbers ( $\mathrm{n}=6$ /genotype; Mann-Whitney $\mathrm{U}$ test). (E) (Left) 2P-LSM of $P V-G F P^{*} N r g 1$-tg and $P V$-GFP control mice at 3 months of age. Depicted are 3D projections-rendered live-imaging stacks of a cortical column $\left(250 \times 250 \times 500 \mu \mathrm{m}\left(0.03125 \mathrm{~mm}^{3}\right) ; 2 \mu \mathrm{m}\right.$ stack interval) from the MZ to layer V. (Right) Quantification of GFP ${ }^{+}$interneurons in layers I-V from 2P-LSM liveimaging stacks reveals an overall loss of cells and more specifically in layers II/III and V. Note that parts of layers $V$ and $V I$ could not be imaged. ( $P V$-GFP ${ }^{*} N r g 1-t g, n=6 ; P V-G F P, \mathrm{n}=5 ;{ }^{*} \mathrm{p}$ $<0.05$; Student's t test).

a significant increase in the steady-state level of phosphorylated ErbB4 (Fig. 9B). Thus, Nrg1-tg mice represent a model for CRD-NRG1 overexpression-mediated ErbB4 hyperstimulation beginning at late embryonic stages.

Interneurons migrate along a chemo-attractive corridor towards the cortex. It has been shown in vitro that CRD-NRG1 provides one of the permissive signals for this migration (Flames et al., 2004), suggesting that interneuron migration could be affected in Nrg1-tg mice. Similar to the loss-of-function study above, we performed chromogenic immunostainings for GAD67 on coronal paraffin sections of the somatosensory cortex and hippocampus (bregma -1.7) of Nrg1-tg mice and WT controls at P14. The analysis revealed minor changes in the cortical distribution of GAD $67^{+}$cells in Nrg1-tg mice, as we found a reduced number of GAD67 ${ }^{+}$cells in the cortical layer IV, but no change in the total number of hippocampal interneurons (Fig. 9C). We also quantified the number of neocortical and hippocampal $\mathrm{PV}^{+}$interneurons in chromogenic stainings for parvalbumin and found no significant changes in $\mathrm{PV}^{+}$ interneuron numbers for both regions in Nrg1-tg mice at P14 (Fig. 9D).

To visualize $\mathrm{PV}^{+}$interneurons in vivo, we crossbred Nrg1-tg mice to PV-GFP transgenic mice that express GFP under control of regulatory sequences of the $P V$ gene (Meyer et al., 2002). In vivo imaging of $P V$-GFP*Nrg1-tg double-transgenic mice at the age of 3 months by two-photon laser scanning microscopy (2P-LSM) revealed a lower number of $\mathrm{GFP}^{+}$cells in cortical layers II/III and $\mathrm{V}$ and a reduction in the total number of $\mathrm{GFP}^{+}$cells in the cortex by $\sim 20 \%$ compared to PV-GFP single transgenic controls (Fig. 9E). These findings were confirmed in a second transgenic mouse line (HA-Nrg1-tg), which expresses a N-terminally hemagglutinin (HA) epitope-tagged variant of CRD-NRG1 under control of the same Thy1.2 cassette as in Nrg1-tg mice (Velanac et al., 2011; Fig. 10A; and data not shown). 
Taken together CRD-NRG1 serves only minor permissive functions, as cortical layering of $\mathrm{GAD} 67^{+}$interneurons is only mildly altered at P14. However, chronic overexpression of CRD-NRG1 and hyperstimulation of ErbB4 affects the survival of $\mathrm{PV}^{+}$interneurons or the expression of the parvalbumin promoter in 3 months old $P V$ GFP*NRG1-tg and PV-GFP*HA-NRG1-tg mice.

Next, we tested whether hyperstimulated CRD-NRG1/ErbB4 signaling causes behavioral dysfunctions. The neuromuscular junction in Nrg1-tg mice is severely compromised (W.J. Thompson, personal communication), which prevents behavioral analyses. Thus, we examined behavior in HA-Nrg1-tg mice (Velanac et al., 2011; Fig. 10A). Similar to Nrg1-tg mice, we observed HA-NRG1 expression all over the brain, in the neocortex and hippocampus, but also in subcortical regions, such as the thalamus, striatum and amygdala (Fig. 10B). Fluorescent immunostaining demonstrated that HA-NRG1 accumulates on the surface of cortical projection neurons, but was absent from GABAergic interneurons, GFAP ${ }^{+}$astrocytes and Olig2 ${ }^{+}$ oligodendrocytes (Fig. 10C).

To assess general locomotor activity and anxiety, we performed the open field test. Mice are neophobic and find open spaces aversive, thus normal mice prefer to stay close to the walls of the open field arena (thigmotaxis). Time spent in the corners or the center of the arena can be quantified as a measure of anxiety. Mice were placed into the open field arena for 10 minutes and movements were tracked automatically. HA-Nrg1-tg mice showed normal locomotor activity, as the distance travelled in the open field test was unaltered (WT: $44.17 \pm 1.93$, HA-Nrg1-tg: $42.49 \pm 2.21$; Fig. 10E). However, HA-Nrg1-tg mice spent less time in the center of the open field arena (WT: $24.16 \% \pm 3.77 \%, H A-N r g 1-\operatorname{tg}: 10.61 \% \pm 2.13 \%)$ and performed fewer rearings, when compared to WT controls (WT: $56.28 \pm 3.86$, HA-Nrg1-tg: $39 \pm 4.64$ ), suggesting diminished exploratory behavior. In addition they displayed more frequent defecation (WT: $1.778 \pm 0.586$; HA-Nrg1-tg: $4.13 \pm 0.72$ ), in line with increased anxiety (Fig. 10E).

Disrupted sensorimotor gating is implicated in the neuropathology of SZ and has frequently been found in rodent models for SZ. Its disruption in SZ may be related to the processes of sensory flooding and cognitive fragmentation and could underlie the positive symptoms of the disease (Braff et al., 2001; Swerdlow et al., 2008). Prepulse inhibition (PPI) is considered a psychometric measure of sensorimotor gating and can be studied both in rodents and humans (Braff and Geyer, 1990). To assess the effect of NRG1 overexpression on sensorimotor gating, we performed the prepulse inhibition test, where HA-Nrg1-tg mice showed a profound PPI deficit in comparison to controls. Moreover, they displayed an increased startle response (WT: $184.9 \pm$ 
A

2HA-CRD-NRG1
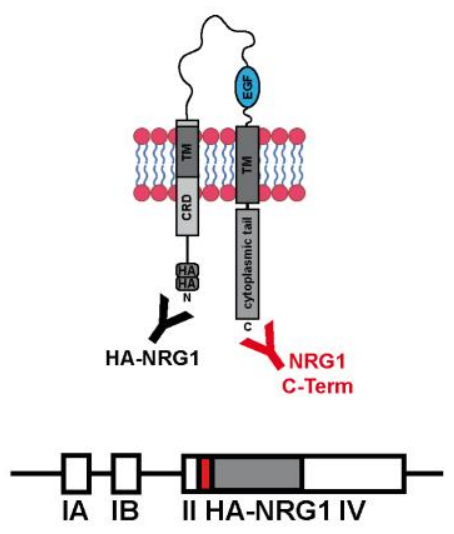

2HA-CRD-NRG1

C

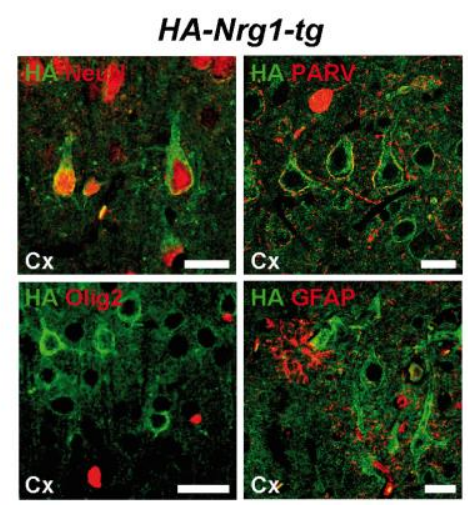

$\mathbf{E}$

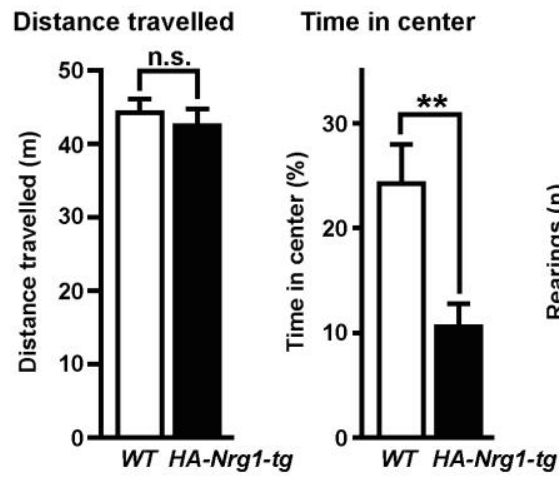

B

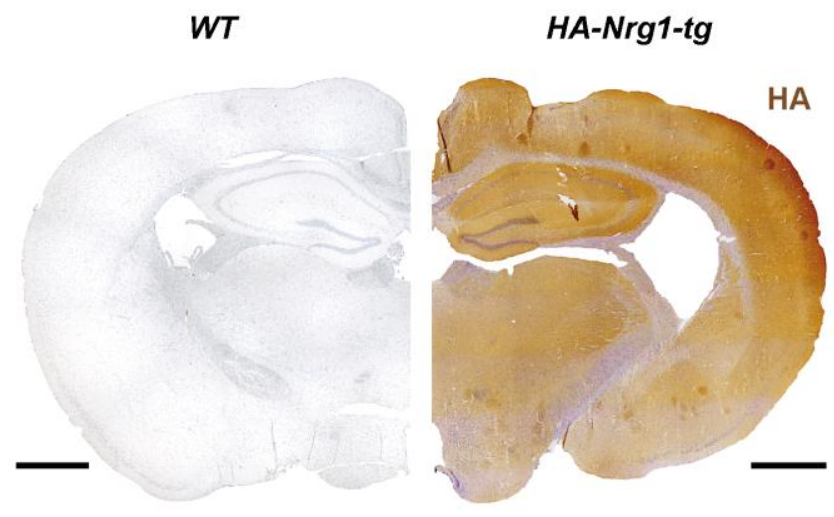

D

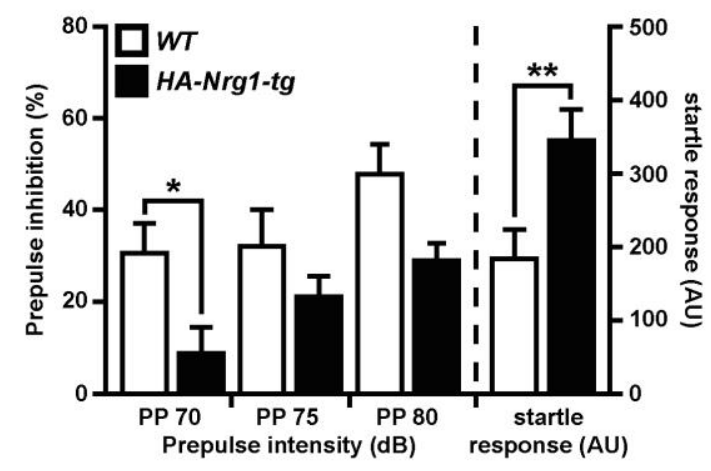

$\mathbf{F}$

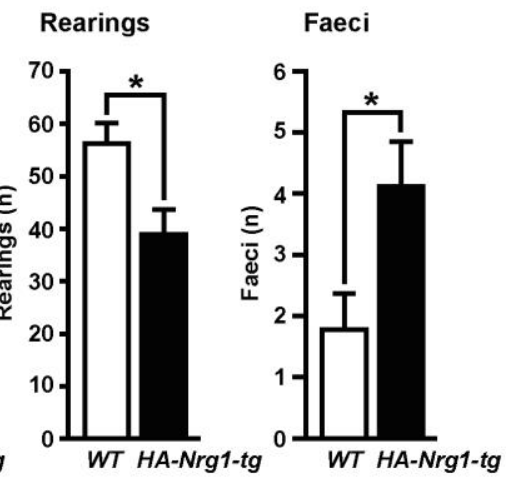

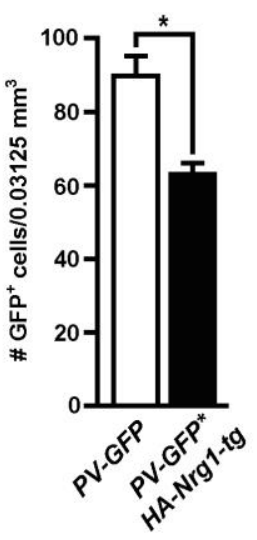

Fig. 10: HA-Nrg1-tg mice exhibit SZ-relevant behavior reduced numbers of interneurons in the cortex. (A) In HA-NRG1-tg mice two HA epitope tags are located at the $\mathrm{N}$-terminus of CRD-NRG1, that can be detected via anti-HA antibody reactivity. The NRG1 CTerm antibody binds to the C-terminal cytoplasmatic tail. CRD, cystein-rich domain; EGF, epidermal growth factor-like domain; HA, hemagglutinin epitope tag; TM, transmembrane domain. Structure of the Thy1.2 transgene cassette (red box, HA epitope; grey box, full-length CRD-NRG1 CDNA; white boxes, exons I-IV of the Thy1.2 gene). (B) Chromogenic NRG1 immunostaining (HA-NRG1) on $5 \mu \mathrm{m}$ coronal paraffin brain sections from wildtype $(W T)$ and HA-Nrg1-tg mice at 4 months of age. Note enlarged lateral ventricle in the HA-Nrg1-tg brain. Scale bars, $1 \mathrm{~mm}$. (C) Fluorescent immunostainings for HA-NRG1 and cell type-specific 
markers (neurons, NeuN; interneurons, PARV; oligodedrocytes, Olig2; astrocytes, GFAP) on coronal brain sections from HA-Nrg1-tg mice (age 4 months) show projection neuron-specific HA-NRG1 overexpression. Scalebars, $25 \mu \mathrm{m}$ (PARV, $20 \mu \mathrm{m}$ ). (D) HA-Nrg1-tg mice show reduced PPI (Effect of genotype $F(1,32)=4.31$; ${ }^{*} p<0.05$; two-way ANOVA; Bonferroni's multiple comparison test), and increased startle responses to $120 \mathrm{~dB}$ pulses (WT, $\mathrm{n}=18 ; H A$ Nrg1-tg mice, $\mathrm{n}=16$; ${ }^{* *} \mathrm{p}<0.01$; Mann-Whitney $\mathrm{U}$ test. $\mathrm{AU}$, arbitrary units, Error bars represent SEM). (E) HA-Nrg1-tg mice display anxiety-like behavior in the open field test. Distance travelled was similar in HA-Nrg1-tg and WT male mice, but HA-Nrg1-tg mice spent less time in the center of the open field arena, performed less rearings and showed increased defecation in the open field test compared to $W T$ controls (age 2-3 months, $p=0.5575$, Mann-Whitney test; error bars represent SEM). (F) Reduced overall number of GFP ${ }^{+}$ interneurons in a cortical column of $250^{*} 250^{*} 500 \mu \mathrm{m}\left(0.03125 \mathrm{~mm}^{3}\right)$ of PV-GFP*HA-Nrg1-tg mice $(\mathrm{n}=2)$ and $P V$-GFP controls $(\mathrm{n}=5)$ obtained by $2 \mathrm{P}$-LSM in vivo imaging $\left({ }^{*} \mathrm{p}<0.05\right.$; Student's $\mathrm{t}$ test).

39.56, HA-Nrg1-tg: $344.9 \pm 42.07$ ) (Fig. 10D).

In summary, CRD-NRG1 overexpression triggers SZ-relevant behavior. Enhanced CRD-NRG1/ErbB4 signaling impacts on anxiety-related pathways in the brain of $H A$ Nrg1-tg mice and impairs sensorimotor gating, indicating that enhanced NRG1/ErbB4 signaling could contribute to SZ pathology.

\subsection{Generation of a conditional CRD-NRG1 transgenic mouse line}

The human NRG1 gene encodes for more than 30 isoforms (Falls, 2003; Mei and Xiong, 2008). Additional complexity of NRG1 signaling is added by posttranslational modifications and dynamic expression patterns in the brain (Liu et al., 2011). The expression of CRD-NRG1 is regulated during development and peaks at E13 and P5 suggesting roles in neural development and in the neonatal critical period (Liu et al., 2011). Nrg1-tg and HA-Nrg1-tg mice show endophenotypes associated with SZ, but the Thy1.2-based transgene cassette offers no chance to manipulate the temporal and spatial aspects of NRG1 overexpression.

To match the complexity of endogenous NRG1 biology and to model different scenarios of NRG1 overexpression, a more sophisticated mouse model was generated by oocyte injection to manipulate cell type-specific, temporal and spatial aspects of NRG1 overexpression. Stop-Nrg1 transgenic mice harbor an expression cassette that is driven by a ubiquitously expressed chicken $\beta$-actin promoter and contains a GFP-Stop cassette flanked by loxP sites followed by the same HA-tagged CRD-NRG1 variant (HA-NRG1) as in HA-Nrg1-tg mice. Crossbreeding Stop-Nrg1 mice to Cre recombinase expressing mouse lines ('driver' lines) results in Cremediated removal of the GFP-Stop cassette and HA-NRG1 expression in the cell type and at the expression start defined by the chosen Cre driver line (Fig. 11A). 


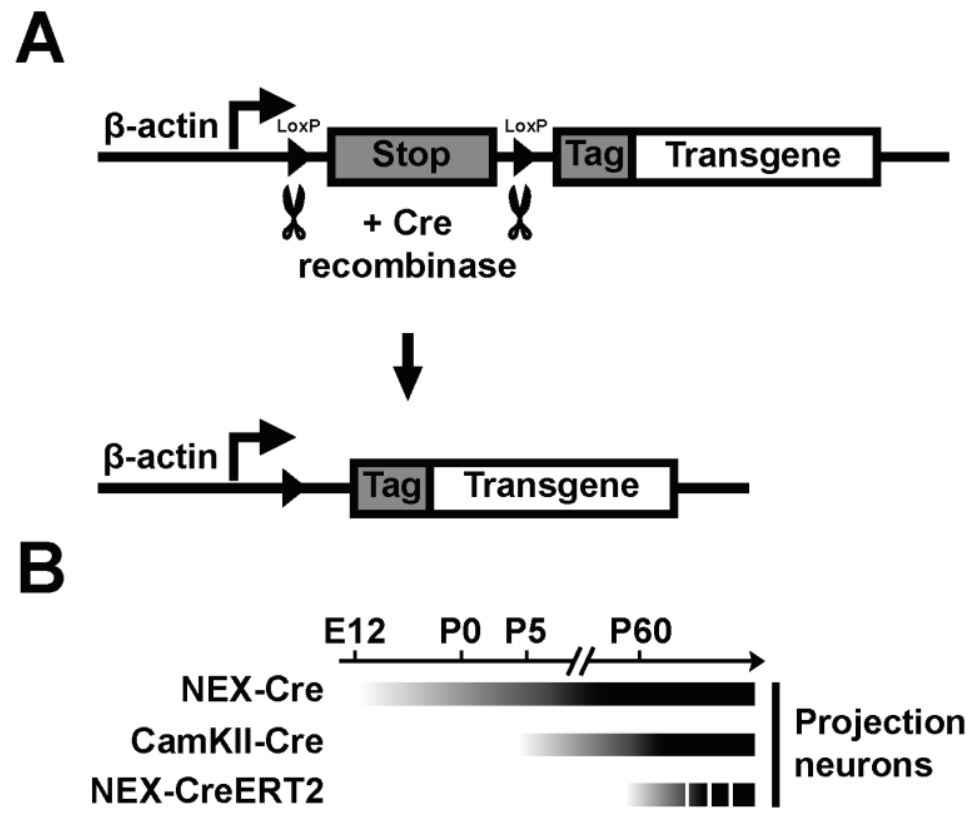

Cre expression

Fig. 11: A $\beta$-actin GFP-Stop-flox transgene cassette allows Cre-mediated transgene expression. (A) Basic principle of the $\beta$-actin Stop-flox transgene cassette used in Stop-Nrg1 mice. Using a fragment of the chicken $\beta$-actin promoter, a GFP-Stop cassette (Stop) is expressed, that is flanked by loxP sides. Upon Cre recombinase-mediated excision of the GFP-Stop-flox cassette, a tagged downstream transgene is expressed. (B) Different Cre driver mouse lines define the celltype-specificity and start of recombination in Stop-Nrg1 double transgenic mice. NEX-Cre driver mice enable early embryonic cortical projection neuron-restricted overexpression starting at E12 (Goebbels et al., 2006), while CamKII-Cre driver mice express Cre recombinase in cortical projection neurons starting at P5 (Minichiello et al., 1999). NEX-CreERT2 driver mice enable tamoxifen-induced acute stimulation of Cre recombination and transgene expression in mice of all stages (Agarwal et al., 2011).

Since CRD-NRG1 is mainly expressed by cortical projection neurons (Liu et al., 2011), we chose NEX-Cre mice harboring Cre recombinase as a 'knock-in' into the NeuroD6 locus as a driver line for Cre-mediated recombination in cortical projection neurons starting at E12 (Goebbels et al., 2006), and CamKII-Cre driver mice with a similar expression pattern as in NEX-Cre mice, but a recombination onset at P5 (Minichiello et al., 1999). These two Cre driver lines allow the separation of embryonic from postnatal aspects of NRG1 signaling. A third mouse line (NEXCreERT2) represents a modification of the original NEX-Cre driver line and expresses a tamoxifen-inducible variant of Cre recombinase (CreERT2). Administration of tamoxifen, a synthetic estrogen receptor (ER) ligand, induces the dissociation of CreERT2 from HSP90, nuclear import of CreERT2, and the sitespecific recombination of loxP site-flanked target genes, enabling tamoxifen induced induction of HA-NRG1 overexpression at adult stages (Fig. 11B) (Agarwal et al., 2011). 
A
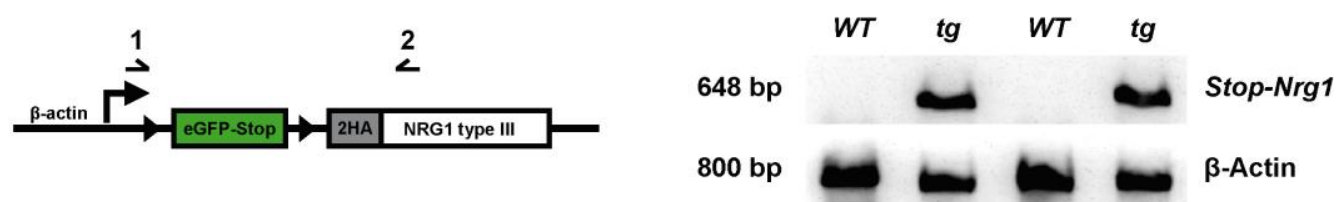

B

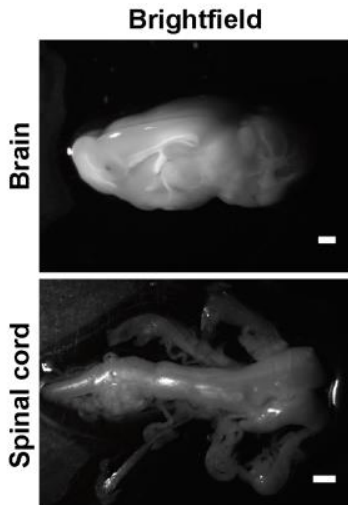

Fluorescence
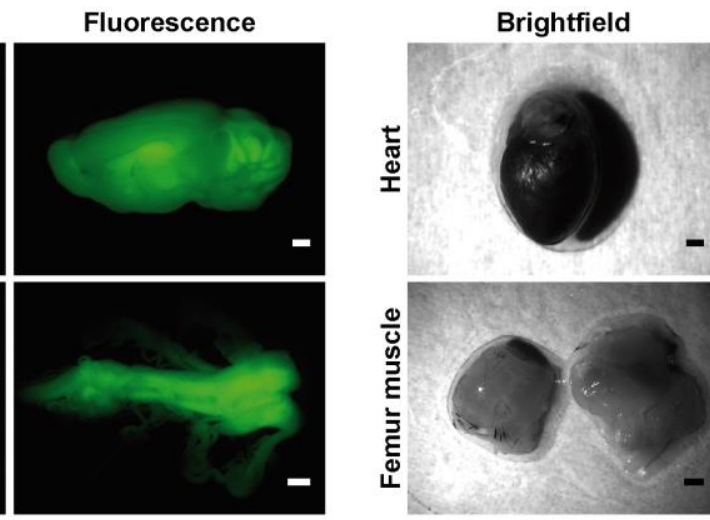

Fluorescence
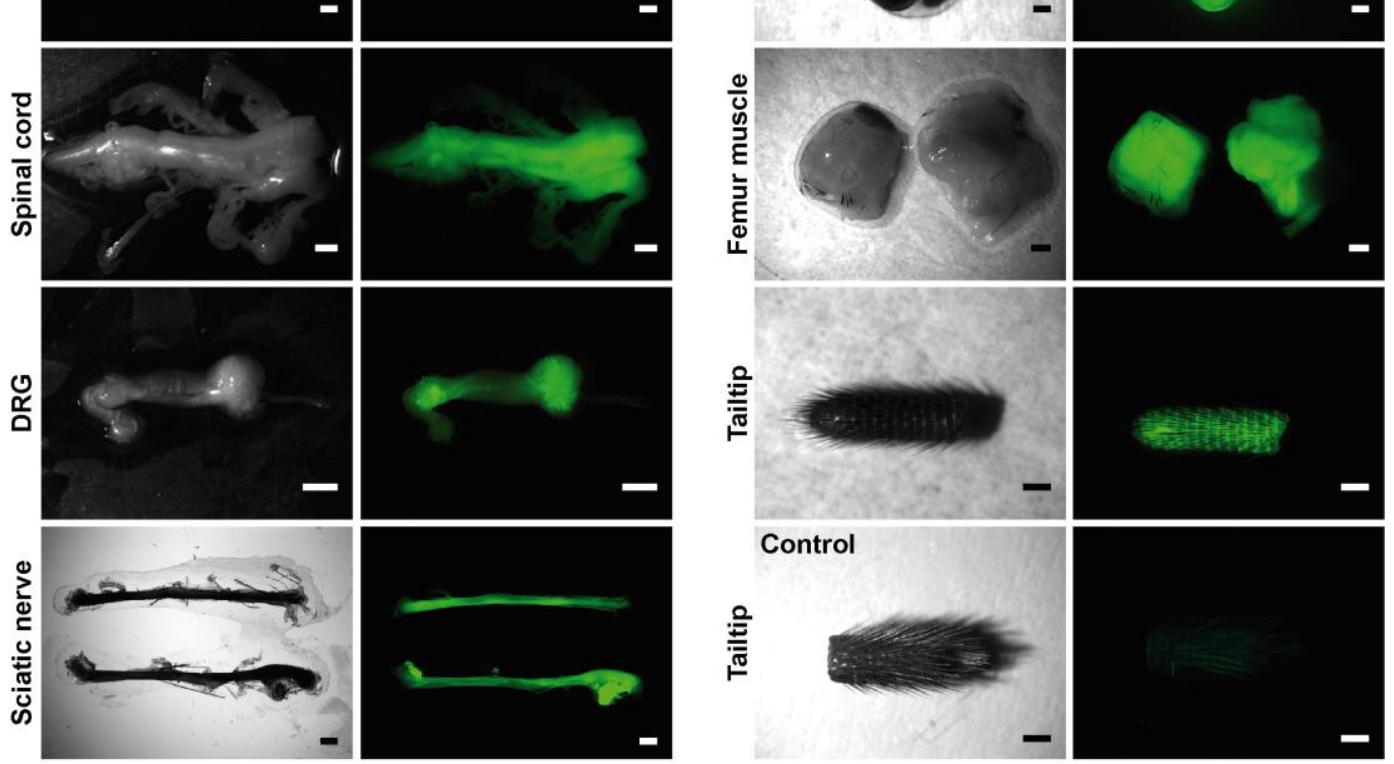

Fig. 12: Stop-Nrg1 mice express the $\beta$-actin GFP-Stop-flox transgene in virtually all tissues. (A) (left) Structure of $\beta$-actin GFP-Stop-flox HA-CRD-NRG1 transgene cassette used in Stop-Nrg1 mice with location of genotyping primers (1 and 2). (right) Genotyping PCR on tail biopsy genomic DNA shows genomic insertion of the Stop-Nrg1 cassette in Stop-Nrg1 mice (tg) compared to $W T$ littermates. $\beta$-Actin PCR was used as DNA quality control. (B) Stop-Nrg1 mice display green fluorescence in virtually all tissues due to GFP expression in the GFP-Stop-flox cassette. Brightfield and epifluorescence images of organs of Stop-Nrg1 mice and a tail biopsy of a $W T$ littermate. Scale bars, $1 \mathrm{~mm}$ (brain, spinal cord, dorsal root ganglia (DRG), heart and tailtip), $2 \mathrm{~mm}$ (femur muscles).

Injection of the Stop-Nrg1 transgene cassette into C57bl6n oocytes produced one founder mouse line (Fig. 12A). Stop-Nrg1 mice were viable and produced offspring according to Mendelian ratios. Since the Stop-Nrg1 transgene harbors a GFPexpressing Stop cassette, transgene expression (in the absence of Cre recombinase) can be assessed by fluorescent light microscopy. When examined under fluorescent excitation all tested tissues were green fluorescent (Fig. 12B), suggesting that the $\beta$-actin promoter is expressed in most tissues. This also enabled ,phenotyping' of Stop-Nrg1 mice using tailtip biopsies instead of genotyping PCR. 
To examine which neural cell types in the brain of Stop-Nrg1 mice express the transgene cassette, we performed fluorescent co-immunostainings on coronal cryosections (Bregma -1.7) from Stop-Nrg1 mice for GFP and cell type-specific markers. Confocal laser microscopy revealed a predominant expression of the Stop-Nrg1 transgene in neurons and oligodendrocytes, as shown by co-staining for GFP and neuronal marker NeuN and oligodendroglial marker Olig2, respectively (Fig. 13A, B). Co-immunostaining for GFP and the projection neuron marker neurogranin (NRGN) and the interneuronal marker GAD67 revealed that the Stop-Nrg1 cassette was predominantly expressed by projection neurons (Fig. 6A). In contrast, only few GAD67 ${ }^{+}$interneurons expressed the Stop-Nrg1 cassette (Fig. 13A). Among glial cells, Stop-Nrg1 was preferentially expressed in oligodendrocytes, whereas only few astrocytes and microglia expressed the transgene (Fig. 13B), showing that the $\beta$ actin promoter was not ubiquitously expressed on the cellular level. Outside the brain, spinal cord motoneurons showed prominent Stop-Nrg1 expression (Fig. 13B).

\subsection{NEX-Cre mediated recombination of Stop-Nrg1 restricts HA-NRG1 expression to cortical projection neurons}

To model cortical HA-NRG1 overexpression (Fig. 14A), we crossbred Stop-Nrg1 mice to NEX-Cre driver mice, in which Cre is selectively expressed by projection neurons of the neocortex and hippocampus (Goebbels et al., 2006; Fig. 14A). Cre expression starts at E12, allowing for early embryonic initiation HA-NRG1 overexpression (Fig. 14A). Western blot analysis of hippocampal protein lysates showed a pronounced overexpression of HA-NRG1 in 4 months old Stop-Nrg $1{ }^{*} N E X$ Cre mice compared to parental controls (Stop-Nrg1 single transgenic and NEX-Cre heterozygous mice) (Fig. 14B). As indicated by increased expression of a $\sim 60 \mathrm{kDa}$ CTF, transgene-derived full length HA-NRG1 was normally processed in the stalk region. In addition, western blot analysis with the phospho-ErbB4 antibody (Tyr1284) confirmed sustained ErbB4 hyperphosphorylation in the hippocampus of StopNrg1*NEX-Cre mice (Fig. 14B).

Chromogenic immunostaining on coronal paraffin sections (bregma -1.7) confirmed that HA-NRG1 expression was restricted to neocortex, hippocampus, amygdala and a few hypothalamic neurons in Stop-Nrg1*NEX-Cre mice mimicking the recombination pattern in NEX-Cre mice (Fig. 14C). In contrast to HA-Nrg1-tg mice (compare Fig. 10B), no HA-NRG1 expression was detected in subcortical regions, e.g. striatum or thalamus. In the dentate gyrus, only the outer granule cells 
A
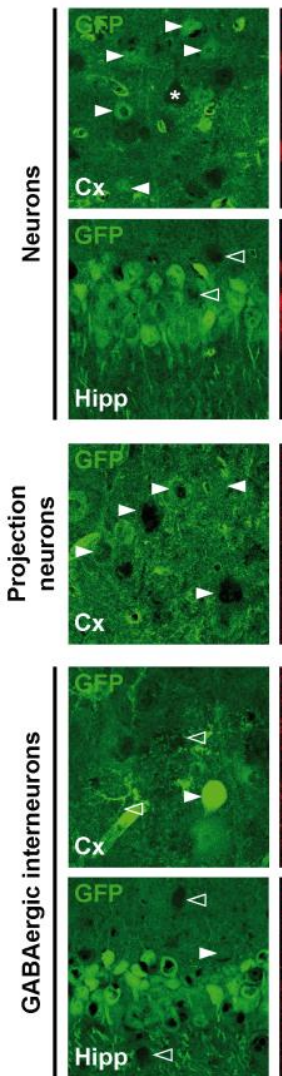
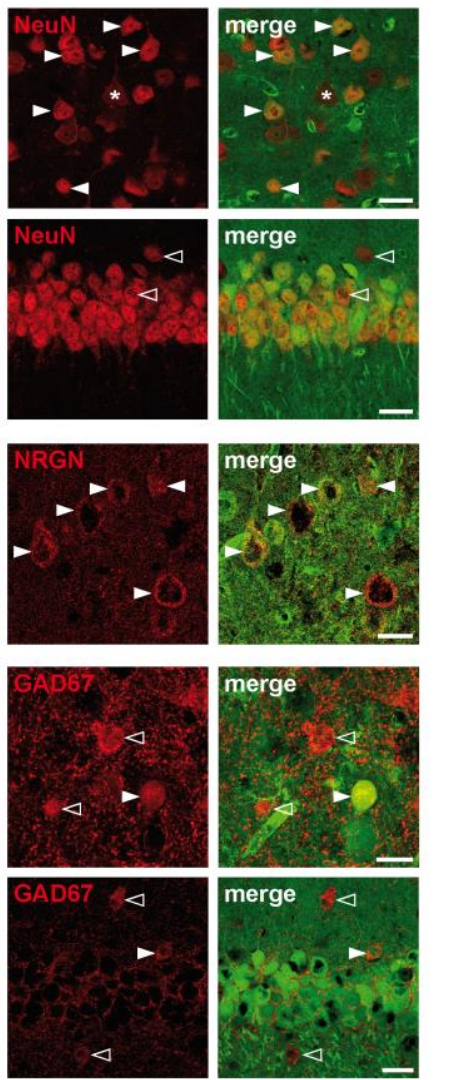

B
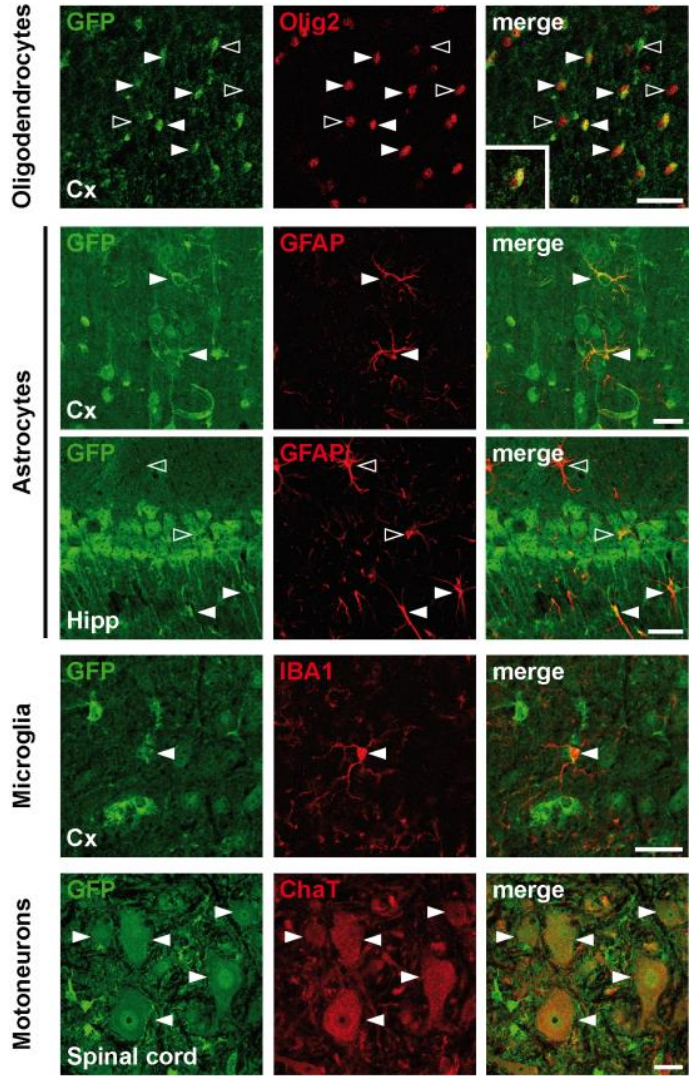

Fig. 13: The Stop-Nrg1 transgene is predominantly expressed in projection neurons. (A) Confocal images of immunostaings on $14 \mu \mathrm{m}$ thick coronal Cryo sections of brains from Stop-Nrg1 mice at 3 months of age stained for neuronal markers. GFP fluorescence coimmunostained for NeuN reveals expression of the Stop-Nrg1 transgene in neurons of the cortex and hippocampus of Stop-Nrg1 mice. Expression of Stop-Nrg1 is present in projection neurons (filled arrowheads), indicated by collocalization with Neurogranin (NRGN), but only minor expression is found in cortical interneurons (GAD67; empty arrowheads). Scale bars, $20 \mu \mathrm{m}$ (NeuN, GAD67 Hipp), $15 \mu \mathrm{m}$ (NRGN, GAD67 Cx). (B) Immunostainings for glial markers reveals expression of the Stop-Nrg1 cassette in oligodendrocytes (Olig2) and partially in astrocytes (GFAP). Co-immunostaining with IBA1 reveals only minor expression in microglia. Ventral horn spinal cord motoneurons (ChaT) express the $\beta$-actin GFP-Stop-flox transgene cassette. Scale bars, $25 \mu \mathrm{m}, 20 \mu \mathrm{m}$ (GFAP Cx).

expressed HA-NRG1 (Fig. 14C), in line with transient Cre expression in postmitotic granule cells in NEX-Cre mice (Goebbels et al., 2006). To validate projection neuronspecific HA-NRG1 overexpression, we co-immunostained coronal paraffin sections (Bregma -1.7) of 4 months old Stop-Nrg1 ${ }^{*} N E X$-Cre mice and controls for HA-NRG1 and the neuronal markers NeuN, NRGN and GAD67 (Fig. 14D). Similar to Thy1.2 HA-Nrg1-tg mice, HA-NRG1 accumulated in the somato-dendritic compartment (compare with Fig. 10C). In addition, co-immunostaining for the HA epitope and the NRG1 CTF almost completely overlapped (Fig. 14D), indicating highly similar subcellular localization of the N-terminal fragment (NTF) and the CTF after proteolytic processing. 
A

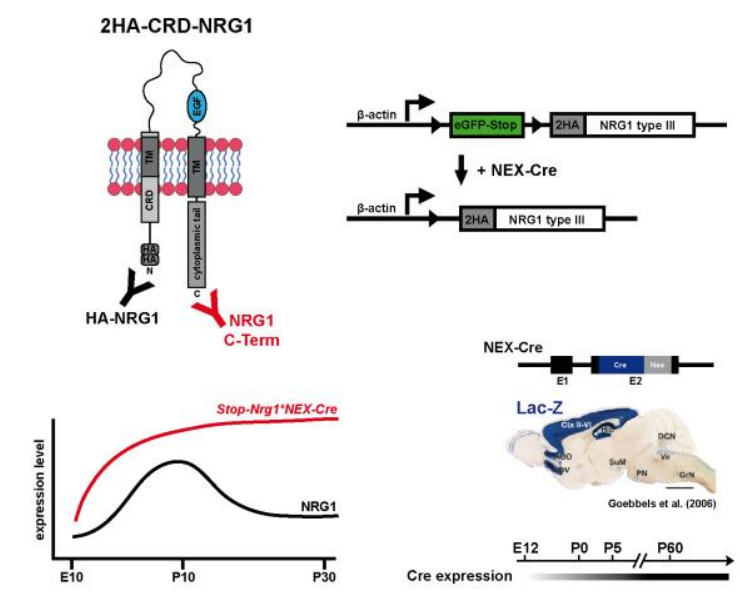

B

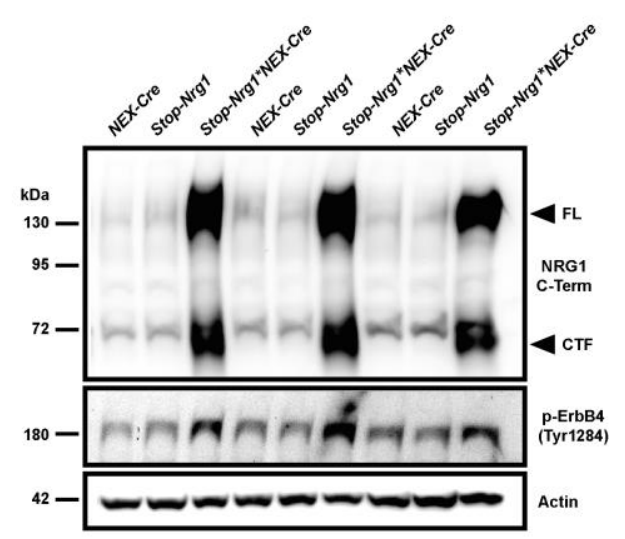

C
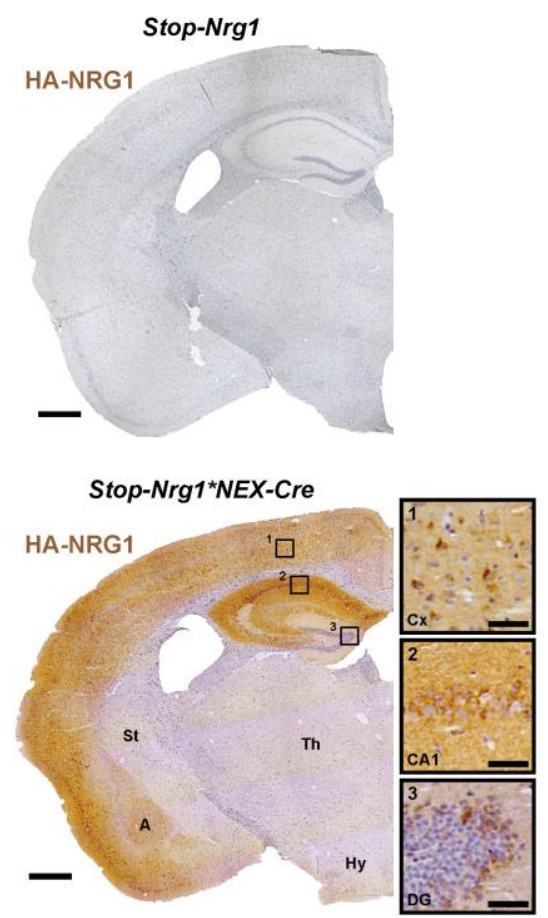

D
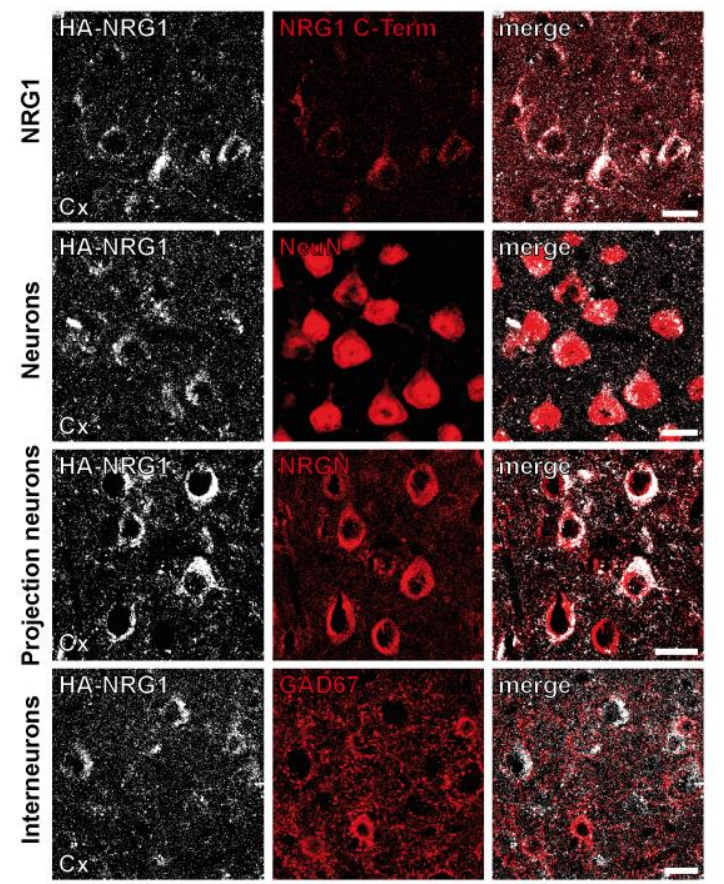

Fig. 14: NEX-Cre-mediated activation of Stop-Nrg1 restricts HA-NRG1 expression to cortical projection neurons. (A) In Stop-Nrg1 mice two HA epitope tags are located the $\mathrm{N}$-terminus of CRD-NRG1, that can be detected via anti-HA antibody reactivity. The NRG1 CTerm antibody binds to the C-terminal cytoplasmatic tail. CRD, cystein-rich domain; EGF, epidermal growth factor-like domain; HA, hemagglutinin epitope tag; TM, transmembrane domain. Structure of Stop-Nrg1 transgene cassette and NEX-Cre-mediated recombination of the GFP-Stop cassette. Schematic expression profile of HA-NRG1 in Stop-Nrg1*NEX-Cre mice. Endogenous CRD-NRG1 expression peaks at P10 and returns to low expression levels in adult mice. In Stop-Nrg1*NEX-Cre mice expression of HA-NRG1 starts after Cre-mediated removal of the GFP-Stop cassette at E12 and persists at high levels into adulthood. Structure of the targeting construct used for homologous recombination of Cre into exon 2 of the murine NEX gene in NEX-Cre mice. X-gal histochemistry indicates NEX-Cre activity in cortical projection neurons of a NEX-Cre*R26R-floxLacZ mouse (E1, Exon1; E2, Exon2; Neo, Neomycin resistance cassette; adapted from Goebbels et al., 2006). In NEX-Cre mice Cre expression starts at E12. (B) Western blotting of hippocampal protein lysates with the NRG1 C-Term and p-ErbB4 antibody reveals HA-NRG1 overexpression in Stop-Nrg ${ }^{*} N E X$-Cre mice and chronic ErbB4 hyperphosphorylation (at Tyr1284) at 4 months of age. $\beta$-Actin was used 
as loading control. CTF, NRG1 C-terminal fragment; FL, full length; kDa, kilodalton. (C) Chromogenic immunostaining for HA-NRG1 on coronal paraffin sections reveals cortexrestricted HA-NRG1 overexpression in Stop-Nrg1*NEX-Cre mice, consistent with NEX-Cre expression profile. Expression is present in neurons of the cortex, hippocampus, amygdala, but only few hypothalamic neurons, striatal and thalamic regions. Boxes indicate positions of high magnification images. Note that in NEX-Cre mice only the outer dentate gyrus granule cells are HA-NRG1-positive. Scale bars, $500 \mu \mathrm{m}, 50 \mu \mathrm{m}$ (Zoom). A, Amygdala; Hy, Hypothalamus; St, Striatum; Th, Thalamus. (D) Fluorescent immunostaining on coronal paraffin section of Stop-Nrg1 ${ }^{*} \mathrm{NEX}$-Cre mice for NRG1 and neuronal markers. HA-NRG1 expression is restricted to cortical projection neurons (NRGN, NeuN) but absent from GAD67 ${ }^{+}$ interneurons. The HA-epitope overlaps with the NRG1 C-Term. Scale bars, $20 \mu \mathrm{m}, 15 \mu \mathrm{m}$ (HA-NRG1/NRG1 C-Term).

Taken together, Stop-Nrg1*NEX-Cre mice allow conditional HA-NRG1 overexpression in cortical projection neurons, which leads to sustained hyperstimulation of ErbB4 receptors.

\subsection{Stop-Nrg ${ }^{\star}$ NEX-Cre mice model HA-NRG1 overexpression in the embryonic cortex}

NRG1 has been suggested to play an important role in interneuronal migration (Flames et al., 2004) and the establishment of cortical circuits (Mei and Xiong, 2008). In line with this, the expression of CRD-NRG1 isoforms peaks at early embryonic stages (Liu et al., 2011). Thus, elevated CRD-NRG1 expression during embryonic stages could impact on a variety of developmental processes. By employing NEXCre driver mice, we wanted to model early embryonic overexpression of CRD-NRG1 (Fig. 15A).

To verify expression onset of HA-CRD-NRG1 in Stop-Nrg1*NEX-Cre mice during early embryonic stages, we performed immunohistochemistry on coronal cryosections of embryonic brains at E16 (Fig. 15B). The cryofixation preserved the GFP fluorescence in this approach. Consecutive sections of the cortical plate were immunostained for Cre and HA-NRG1. By confocal microscopy Cre-expressing neurons of the cortical plate and subplate were identified (imaged areas depicted in Fig. 15B), whereas GFP fluorescence was markedly reduced in Cre-expressing regions of Stop-Nrg1*NEX-Cremice, due to recombination of the GFP-Stop cassette (Fig. 15C). As expected, in cortical areas with Cre expression and concomitantly reduced GFP fluorescence we observed staining for HA-NRG1 (Fig. 15C, D).

Taken together, breeding to Stop-Nrg1*NEX-Cre mice initiates HA-NRG1 overexpression during early embryonic stages, and allows to study the effects of increased CRD-NRG1 expression on embryonic development. 
A

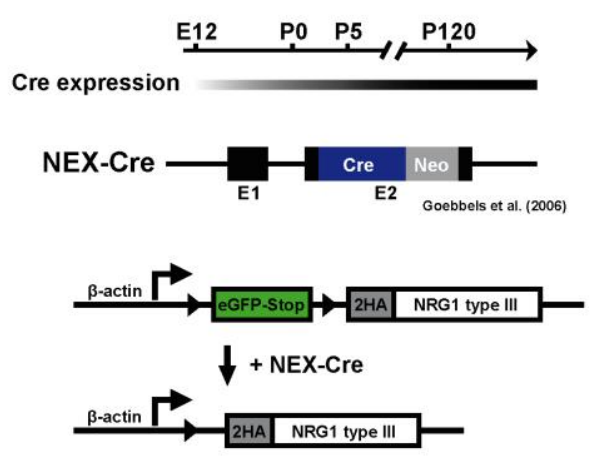

B

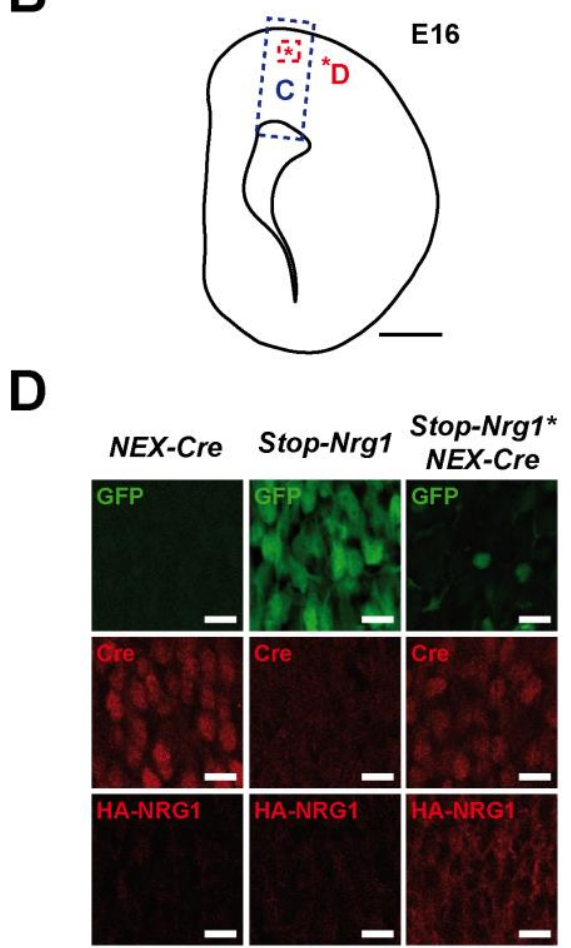

C

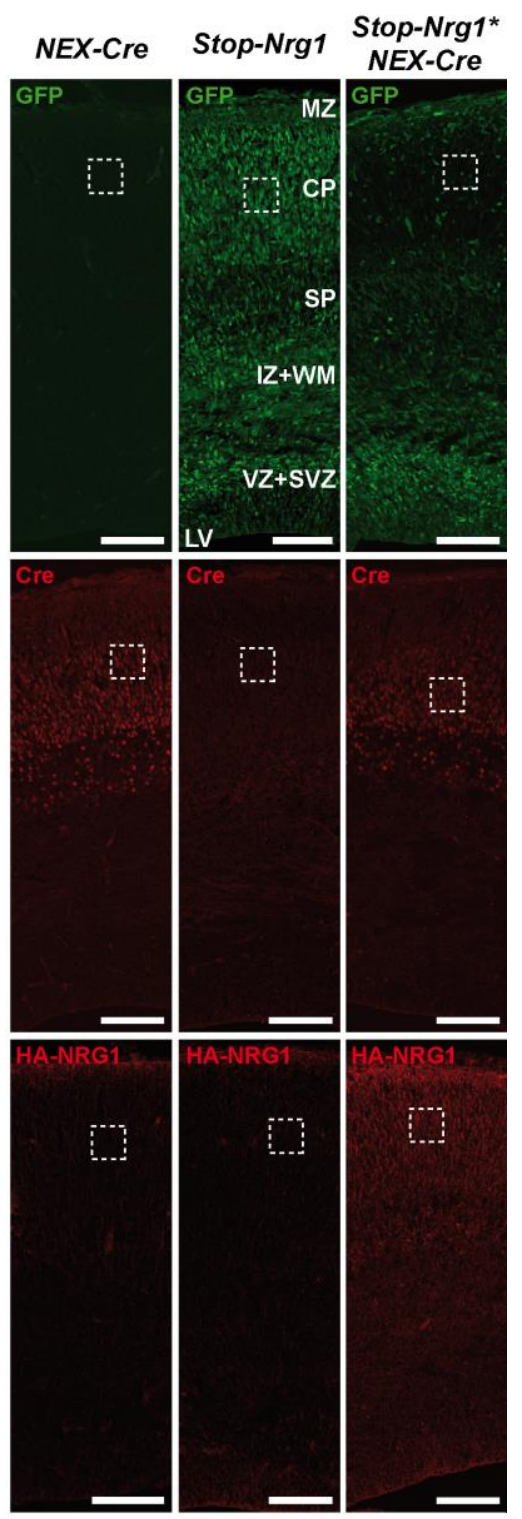

Fig. 15: NEX-Cre-mediated recombination of Stop-Nrg1 allows early embryonic overexpression of HA-NRG1. (A) In NEX-Cre mice Cre expression starts at E12. Structure of the targeting construct used for homologous recombination of Cre into exon 2 of the murine NEX gene in NEX-Cre mice. (E1, Exon1; E2, Exon2; Neo, Neomycin resistance cassette; adapted from Goebbels et al., 2006). Structure of the Stop-Nrg1 cassette and NEX-Cremediated recombination of the GFP-Stop cassette. (B) Schematic drawing of an E16 coronal brain section illustrates the position of overview images shown in $(C)$ (dashed blue square labelled C) and high magnification images shown in (D) (dashed red square labelled with *D). Scale bar, $500 \mu \mathrm{m}$. (C) Immunostainings for GFP, Cre recombinase and HA-NRG1 on consecutive cryosections of Stop-Nrg1 ${ }^{\star} N E X$-Cre and control brains (NEX-Cre and Stop-Nrg1) at E16. In Stop-Nrg1*NEX-Cre embryos GFP fluorescence is reduced in the cortical plate, subplate and intermediate zone due to NEX-Cre-mediated removal of the GFP-Stop cassette, and HA-NRG1 is expressed. Dashed boxes indicate position of high magnification images shown in (D). CP, cortical plate; IZ, intermediate zone; LV, lateral ventricle; $M Z$, marginal zone; SP, subplate; SVZ, subventricular zone; VZ, ventricular zone; WM, white matter. Scale bars, $100 \mu \mathrm{m}$. (D) High magnification images of regions indicated by dashed boxes in (C). Scale bars, $10 \mu \mathrm{m}$. 


\subsection{CamKII-Cre mice enable postnatal overexpression of HA-NRG1 in cortical projection neurons}

$\mathrm{SZ}$ is considered a neurodevelopmental disease and first symptoms usually occur in early adulthood, between 15 and 25 years of age (Sham et al., 1994). Although most of synaptogenesis is completed by two years of age, this process continues until midadolescence in prefrontal and association (temporal and parietal) areas (Huttenlocher, 1979; Giedd et al., 1999). Thus, postnatal dysfunctions of signaling modules, such as NRG1/ErbB4 could lead to abnormalities in cortical development associated with neuropsychiatric disorders, including SZ.

To distinguish embryonic from postnatal effects of abnormal NRG1/ErbB4 signaling, we crossbred Stop-Nrg1 mice to CamKII-Cre driver mice (from now on referred to as CKII-Cre mice), which express Cre recombinase under control of the CaMKII $\alpha$ promoter. Cre activity starts around P5 and is restricted to projection neurons of the cortex and hippocampus, but also some subcortical excitatory neurons of the striatum, thalamus and hypothalamus express Cre recombinase (Minichiello et al., 1999; Fig. 16A). Western blot analysis confirmed HA-NRG1 overexpression in hippocampus protein lysates. Similar to our previous studies, HA-NRG1 was expressed as a full length protein ( $140 \mathrm{kDa})$ and a CTF ( 60 kDa) (Fig. 16B) as a result of proteolytic cleavage in the stalk region. Chromogenic immunostaining showed HA-NRG1 overexpression in the neocortex and hippocampus, but minor expression was also detected in the striatum and thalamus (Fig. 16C). Higher magnification showed that, in contrast to NEX-Cre-mediated HA-NRG1 expression, all dentate gyrus granule cells expressed HA-NRG1 (Fig. 16C: DG). Fluorescent immunostaining confirmed somato-dendritic accumulation and projection neuronspecific HA-NRG1 overexpression (Fig. 16D).

Thus, a comparative analysis of Stop-Nrg ${ }^{*} \mathrm{NEX}$-Cre and Stop-Nrg ${ }^{*} \mathrm{C} K \mathrm{KI}-\mathrm{Cre}$ mice allows to identify possible differences in embryonic versus postnatal effects of HANRG1 overexpression on brain functions. 
A

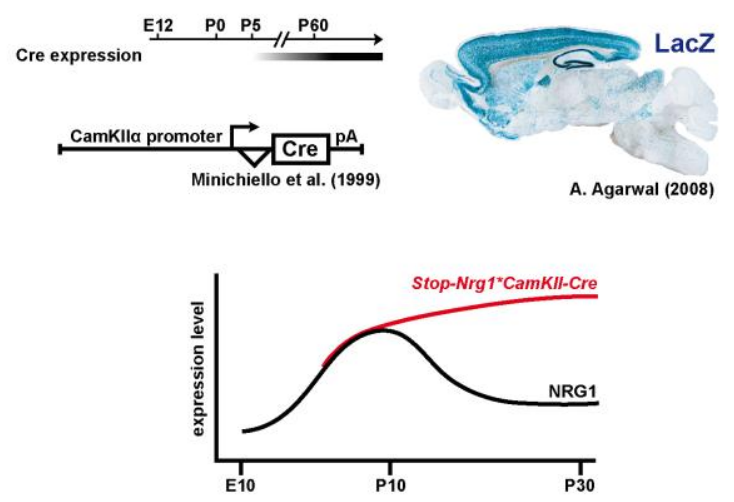

C

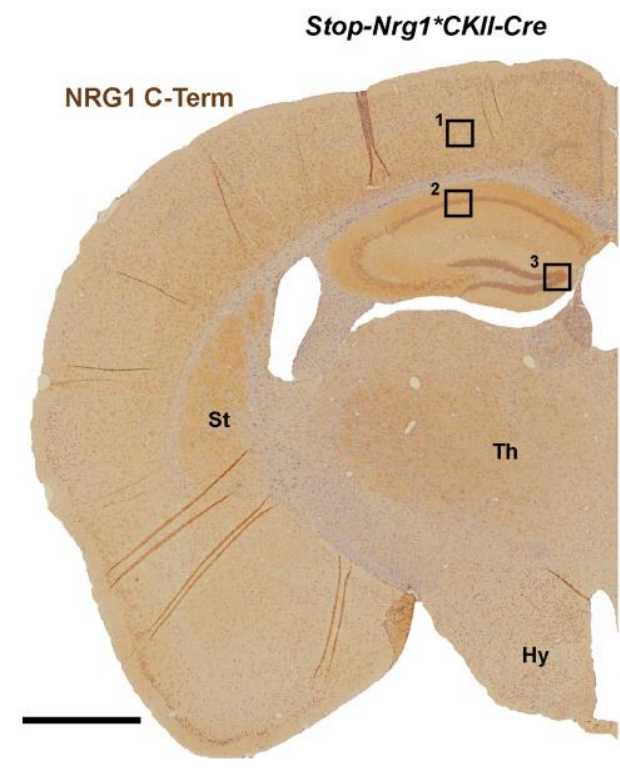

B

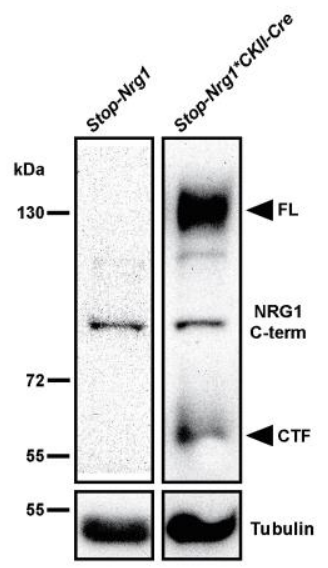

D
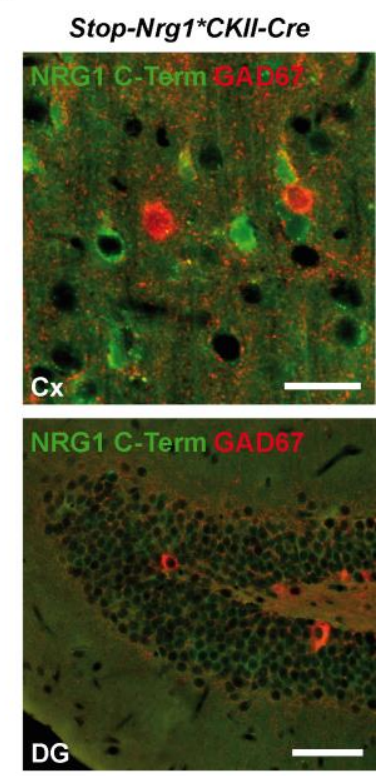

Fig. 16: CKII-Cre-mediated activation of Stop-Nrg1 allows postnatal overexpression of HA-NRG1 in cortical projection neurons. (A) In the CKII-Cre driver line Cre activity begins at P5. Structure of the CKII-Cre transgene used for generation CKII-Cre mice. Triangle represents intronic sequences. pA, polyadenylation signal (adapted from Minichiello et al., 1999). X-gal histochemistry from a CKII-Cre ${ }^{\star} R 26 R$-floxLacZ mouse illustrates Cre activity in cortical neurons (with kind permission of A. Agarwal). CKII-Cre-mediated activation of StopNrg1 allows early postnatal onset of HA-NRG1 overexpression. (B) Western blot analysis of hippocampal protein lysates of Stop-Nrg $1{ }^{*} \mathrm{CKII}-\mathrm{Cre}$ and Stop-Nrg1 control mice at 4 months of age. NRG1 C-Term antibody immunoreactivity reveals HA-NRG1 overexpression as a full length ( 140 kDa, FL) and a C-terminal fragment ( 60 kDa CTF). $\alpha$-Tubulin was used as a loading control. $\mathrm{kDa}$, kilodalton. (C) Chromogenic immunostaining for NRG1 C-Term on a paraffin brain section from a Stop-Nrg $1{ }^{*} \mathrm{CKII}-\mathrm{Cre}$ mouse at 4 months of age reveals overexpression of HA-NRG1 in neurons of the cortex and minor overexpression in subcortical regions, including striatum (St), thalamus (Th) and hypothalamus (Hy). Boxes indicate positions of high magnification images of the cortex (Cx), hippocampal CA1 region (CA1) and dentate gyrus (DG). Note that all DG granule cells express HA-NRG1. Scale bars, $1 \mathrm{~mm}$ (overview), $50 \mu \mathrm{m}$ (high magnifications). (D) Fluorescent immunostainings for NRG1 C-Term and GAD67 on paraffin sections from Stop-Nrg $1{ }^{*} \mathrm{CKII}-\mathrm{Cre}$ at 4 months of age confirm projectionneuron-specific overexpression of HA-NRG1. Cx, cortex; DG, dentate gyrus. Scale bars, $50 \mu \mathrm{m}$. 


\subsection{Absense of brain pathology in Stop-Nrg1*NEX-Cre and Stop- Nrg1 ${ }^{*}$ CKII-Cre mice}

Overexpression of growth- and differentiation factors could lead to abnormal development, neuroinflammation or cell death. Stop-Nrg $1{ }^{\star} N E X-C r e$ and Stop$\mathrm{Nrg} 1{ }^{*} \mathrm{CKII-Cre} \mathrm{mice} \mathrm{displayed} \mathrm{normal} \mathrm{cage} \mathrm{behavior} \mathrm{compared} \mathrm{to} \mathrm{control} \mathrm{littermates.}$ To exclude neuroinflammation or other neuropathology in these mice, we performed chromogenic immunostainings for markers of neuropathology and inflammation on coronal paraffin sections at 4 months and one year of age.

Hematoxylin-Eosin $(\mathrm{H}+\mathrm{E})$ staining revealed no gross abnormalities in brain morphology in both transgenic models (data not shown). Subsequently we tested for astrogliosis or microgliosis by chromogenic immunostainings for GFAP, IBA1 and MAC3 on coronal paraffin sections (bregma -1.7) (Eng and Ghirnikar, 1994; Hanisch and Kettenmann, 2007). Furthermore, we examined brain sections for axonal swellings by staining for amyloid precursor protein (APP), and T-cell infiltration by staining for the T-cell antigen CD3.

Stainings for activated microglia, T-cell infiltration, and axonal swellings showed no signs of pathology in Stop-Nrg1 ${ }^{*} \mathrm{NEX}$-Creand Stop-Nrg1 ${ }^{*} \mathrm{CKII}$-Cre mice at 4 months and 1 year of age compared to controls (Fig. 17). Immunostainings for the astrocytic marker GFAP were quantified with a semi-automated method, which determined the $\mathrm{GFAP}^{+}$area in relation to the region of interest. Hippocampus and fimbria were analyzed as examples for grey and white matter regions, respectively (Fig. 18A). No obvious differences in $\mathrm{GFAP}^{+}$area were observed in the hippocampus of Stop$N r g 1{ }^{*} N E X$-Cre or Stop-Nrg $1{ }^{*}$ CKII-Cre mice. However, the GFAP ${ }^{+}$area in the fimbria at 4 months was significantly larger in Stop-Nrg1 ${ }^{\star} N E X$-Cre $(13.41 \% \pm 1.49)$ than in Stop-Nrg ${ }^{*}$ CKII-Cre mice $\left(5.76 \% \pm 1.84 ;{ }^{*} p<0.05\right)$. This effect however, was not significant when compared to control mice (Fig. 18B). At 1 year of age there was no significant difference detectable in any of the mice tested (Fig. 18C), indicating a non-progressive pathology. Taken together, Stop-Nrg1*NEX-Cre and Stop$\mathrm{Nrg} 1{ }^{*} \mathrm{CKII}-\mathrm{Cre}$ mice showed no signs of progressive astrogliosis (Fig. 18B, C). 
A

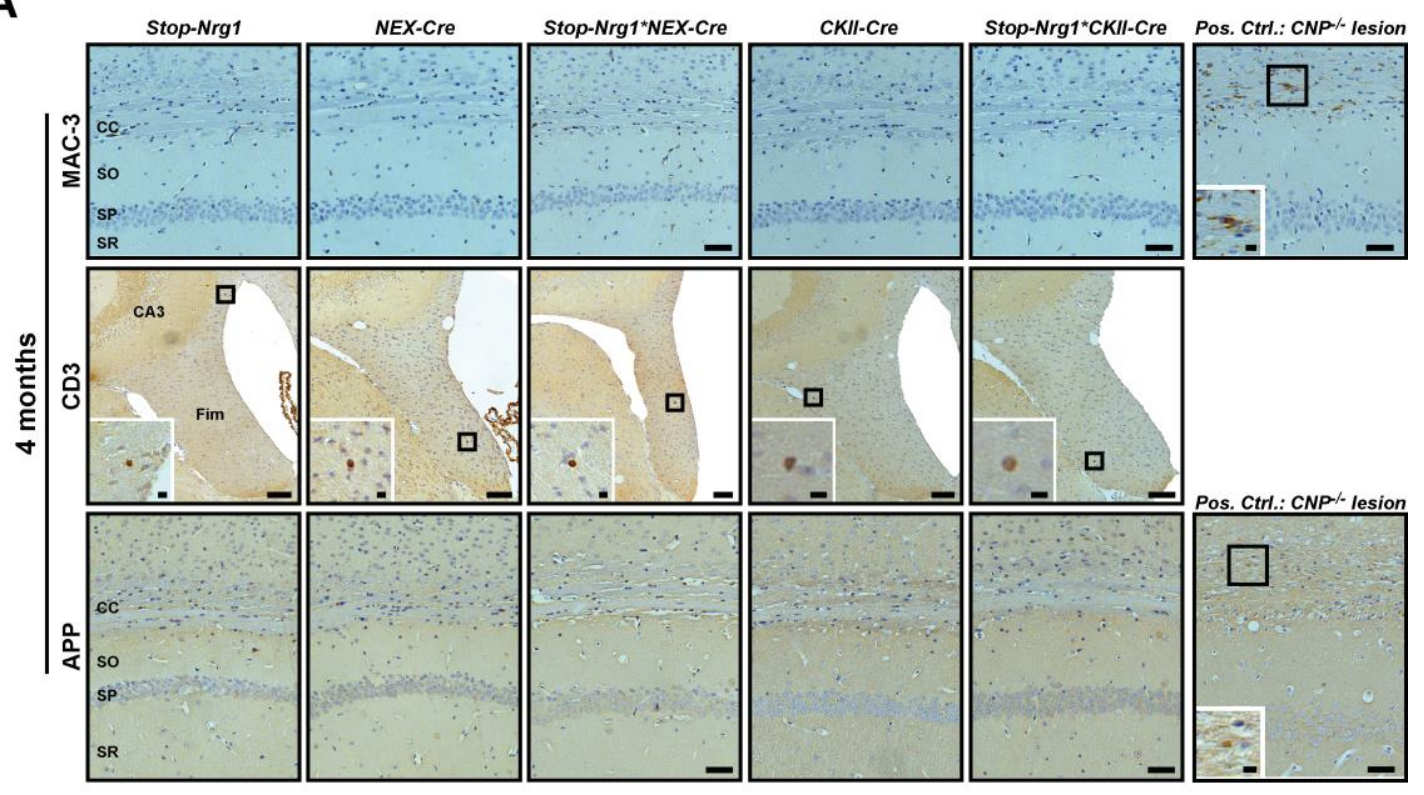

B

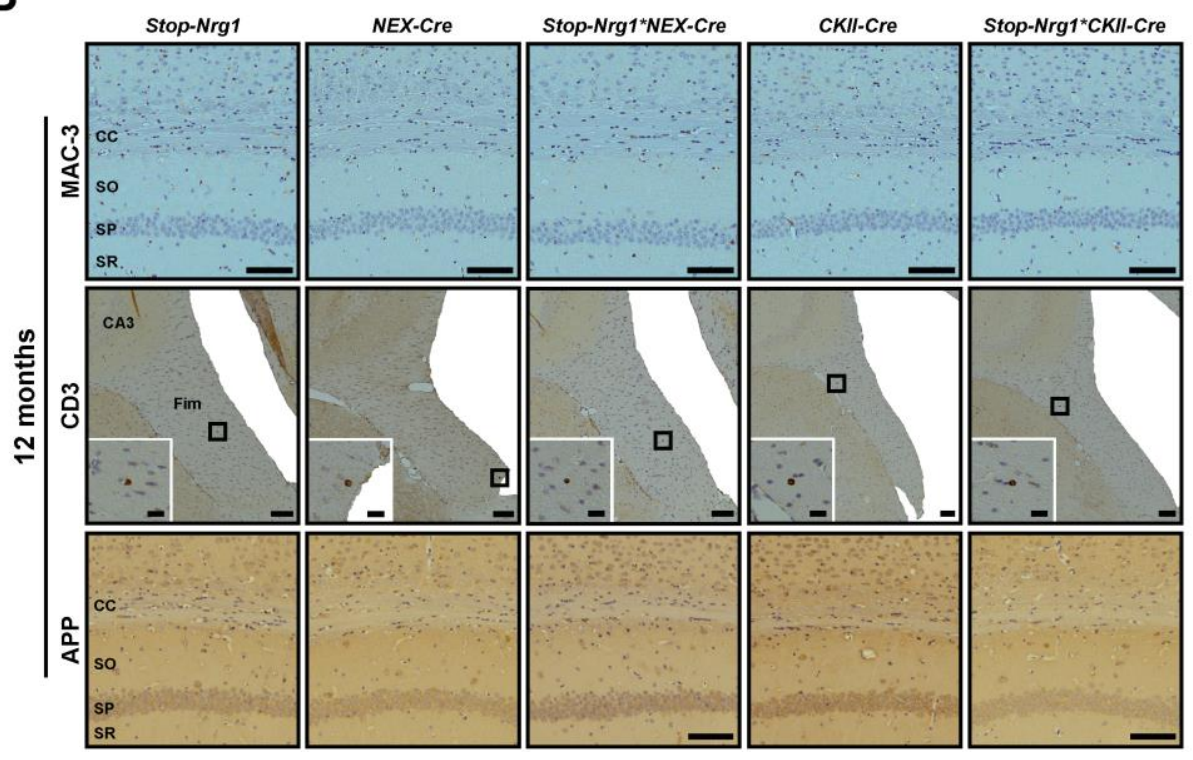

Fig. 17: Stop-Nrg1*NEX-Cre and Stop-Nrg1 ${ }^{*}$ CKII-Cre mice show no signs of neuroinflammation. (A) Chromogenic immunostainings for neuropathology markers on coronal paraffin sections (bregma -1.7) of 4 months old Stop-Nrg ${ }^{*} \mathrm{NEX}$-Cre, Stop-Nrg1 ${ }^{*} \mathrm{CKII-}$ Cre and control mice for activated microglia (MAC-3), T-cell infiltration (CD3) and axonal swellings (APP), reveal no signs of neuropathology or -inflammation. Insets in CD3 stainings show high magnifications of occasionally found $\mathrm{CD}^{+} \mathrm{T}$-cells (indicated by boxes in overviews). Sections of $\mathrm{CNP}^{-/}$mice, which received cryo lesions, were used as positive controls (Wieser et al., 2013). Scale bars, $50 \mu \mathrm{m}$ (overviews), $100 \mu \mathrm{m}$ (CD3), $10 \mu \mathrm{m}$ (insets). (B) Sustained HA-NRG1 overexpression does not lead to neuroinflammation in aged Stop$\mathrm{Nrg} 1^{*} \mathrm{NEX}$-Cre and Stop-Nrg $1^{*} \mathrm{CKII}-\mathrm{Cre}$ mice. Chromogenic immunostainings on coronal paraffin sections from 1 year old mice for MAC-3, CD3 and APP, as in (A). CA3, hippocampal CA3 region; CC, corpus callosum; Fim, fimbria; SO, stratum oriens; SP, stratum pyramidale; $\mathrm{SR}$, stratum radiatum. Scale bars, $100 \mu \mathrm{m}$ (overviews), $20 \mu \mathrm{m}$ (insets).

Likewise, no changes in activated microglia were found in immunostainings for MAC3 (Fig. 17). To determine microglia numbers, immunostaining for IBA1 was 
performed (Fig. 19). Similar to GFAP immunostainings, a semi-automated quantification of chromogenic IBA1 immunostainings was conducted. Cortex and hippocampus were quantified as examples for grey matter, and the corpus callosum as an example for white matter regions (quantified regions are illustrated in Fig. 19A, C). At 4 months of age no difference in IBA $1^{+}$area was detectable in both transgenic models. A trend for increased IBA $1^{+}$area in the cortex of NEX-Cre controls and StopNrg $1^{\star} N E X$-Cre mice was not significant (Fig. 19B). Also at 1 year of age no significant differences in IBA $1^{+}$area were detected in cortex and hippocampus of Stop-Nrg1 ${ }^{*} \mathrm{NEX}$-Cre and Stop-Nrg $1{ }^{*} \mathrm{CKII}$-Cre mice. However, there was an increase in IBA $1^{+}$area in the hippocampus of NEX-Cre control mice $(7.49 \% \pm 1.34)$ compared to CKII-Cre and Stop-Nrg $1{ }^{*}$ CKII-Cre mice (CKII-Cre: $3.03 \% \pm 0.56$; Stop-Nrg ${ }^{*}$ CKIICre: $\left.3.23 \% \pm 0.35 ;{ }^{*} p<0.05\right)$, and Stop-Nrg1 mice $\left(2.95 \% \pm 0.29 ;{ }^{*} p<0.01\right)$, but not to Stop-Nrg1*NEX-Cre mice (Mean: $4.46 \% \pm 1.62$ ), indicating an effect of NEX haploinsufficiency (Fig. 19D).

Taken together, chronically elevated HA-NRG1 expression has no potent neurodegenerative or -inflammatory effects when overexpression starts at postnatal or even embryonic stages. However, NEX (NeuroD6) haploinsufficiency in heterozygous $N E X$-Cre driver mice is associated with mildly increased microgliosis in the hippocampus of aged mice. HA-NRG1 overexpression in Stop-Nrg1 ${ }^{*} N E X$-Cre mice seemed to be beneficial and counteracted this effect. 
A
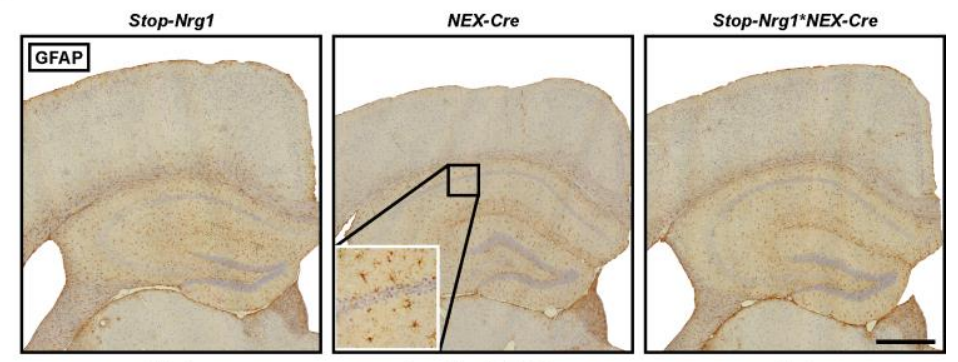

CKII-Cre

Stop-Nrg1*CKII-Cre

quantified areas
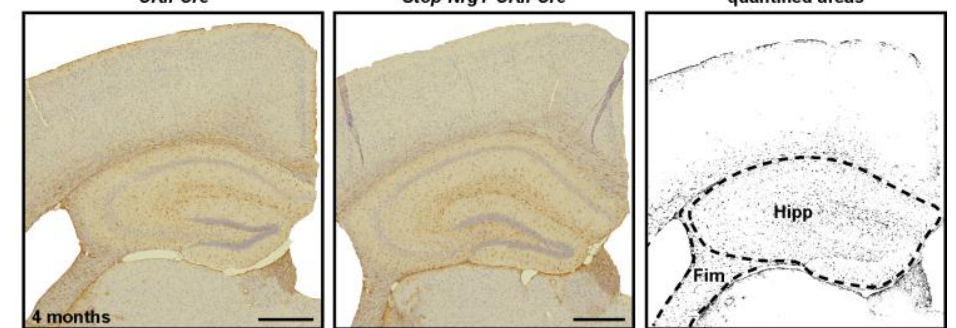

B
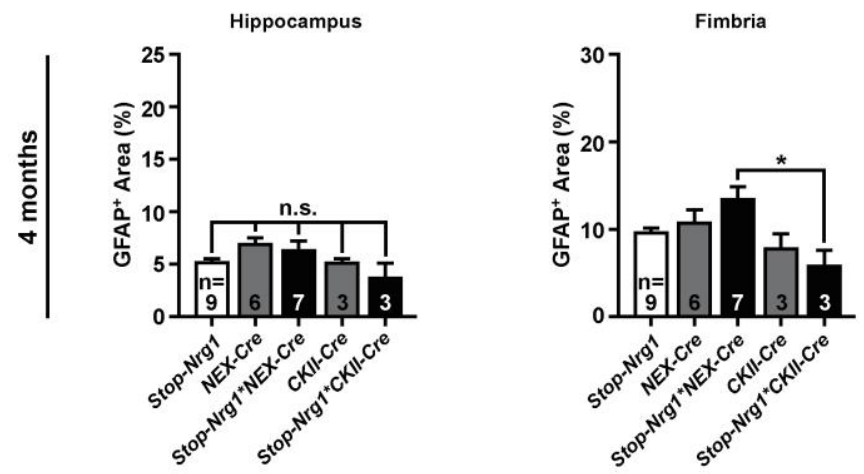

C
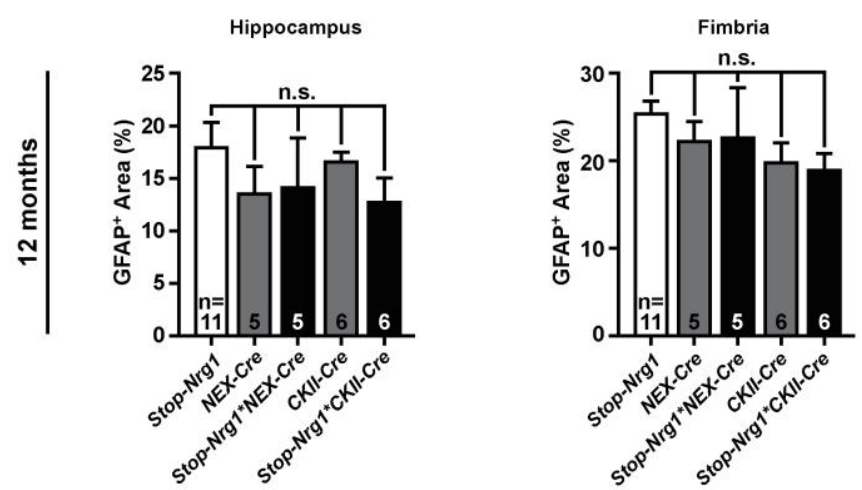

Fig. 18: Stop-Nrg1*NEX-Cre and Stop-Nrg1 ${ }^{\star}$ CKII-Cre mice show no signs of astrogliosis. (A) Chromogenic immunostaining for astrocytes (GFAP) on coronal paraffin sections from 4 months old Stop-Nrg1*NEX-Cre, Stop-Nrg1 ${ }^{*} \mathrm{CKII}-\mathrm{Cre}$ and control mice (bregma -1.7). Dashed lines mark quantified areas. Square indicates position of high magnification image. Fim, fimbria; Hipp, hippocampus. Scale bars, $500 \mu \mathrm{m}$. (B) Semiautomated quantification of GFAP ${ }^{+}$area in hippocampus and fimbria of Stop-Nrg 1 ${ }^{*} \mathrm{NEX}$-Cre, Stop- $\mathrm{Nrg} 1{ }^{*} \mathrm{CKII}-\mathrm{Cre}$ and control mice at 4 months of age reveals a significant increase in $\mathrm{GFAP}^{+}$area in the fimbria of Stop-Nrg $1^{*} \mathrm{NEX}$-Cre mice compared to Stop-Nrg $1{ }^{*} \mathrm{CKII}-\mathrm{Cre}$ mice, that, however, does not differ significantly from control mice. $\left({ }^{*} p<0.05\right.$, one-way ANOVA; Bonferroni's multiple comparison test; n.s., not significant; $n$-numbers indicated in the graphs). (C) Semi-automated quantification of GFAP ${ }^{+}$area in hippocampus and fimbria of Stop-Nrg ${ }^{*} \mathrm{NEX}$-Cre, Stop-Nrg1 ${ }^{*} \mathrm{CKII}-\mathrm{Cre}$ and control mice at 1 year of age confirms absence of astrogliosis. (One-way ANOVA with Bonferroni's multiple comparison test; n.s., not significant; $n$-numbers indicated in the graphs). 
A

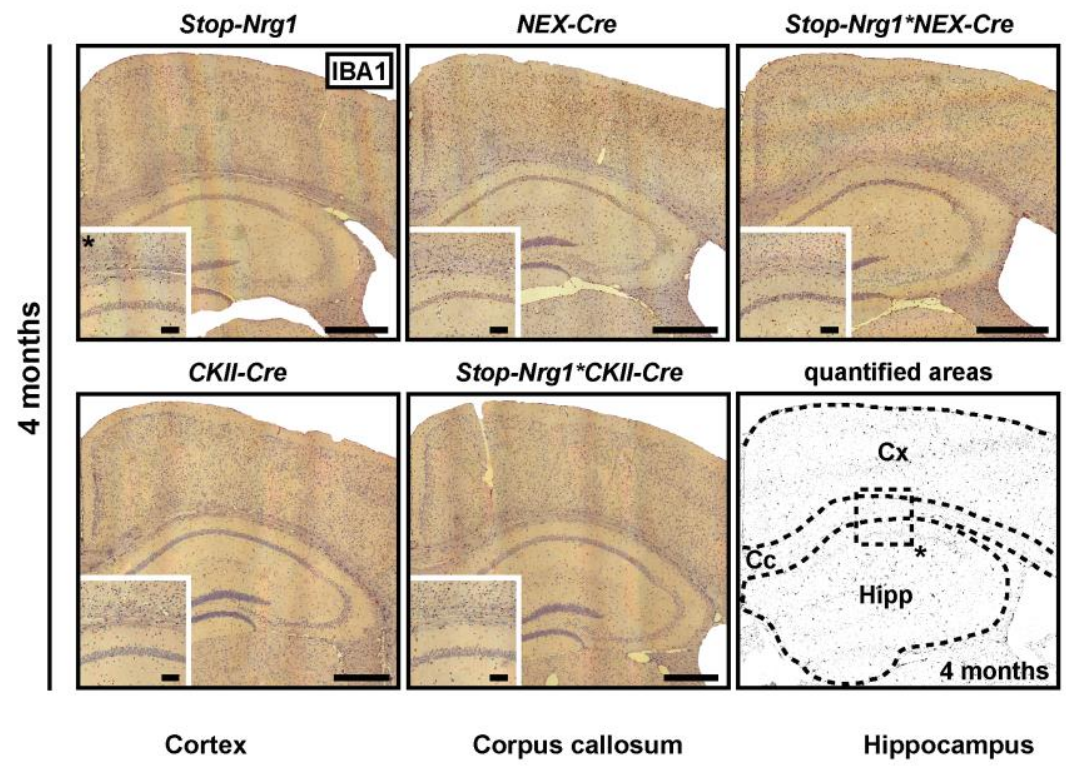

B
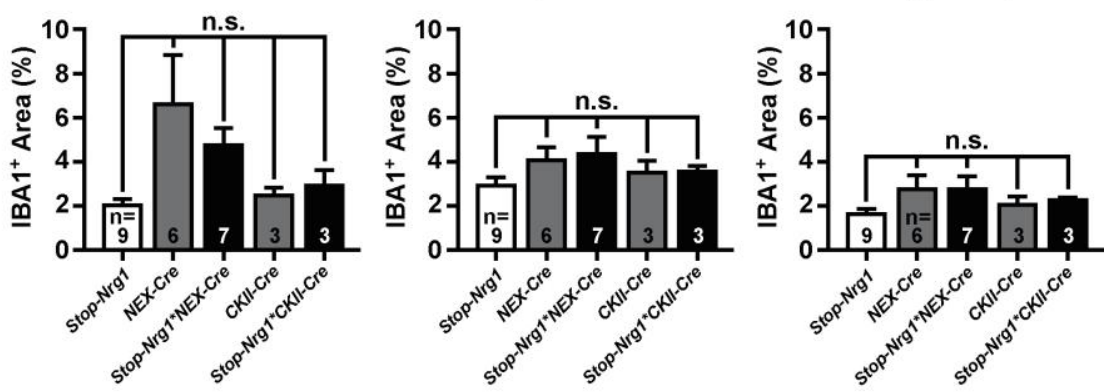

C

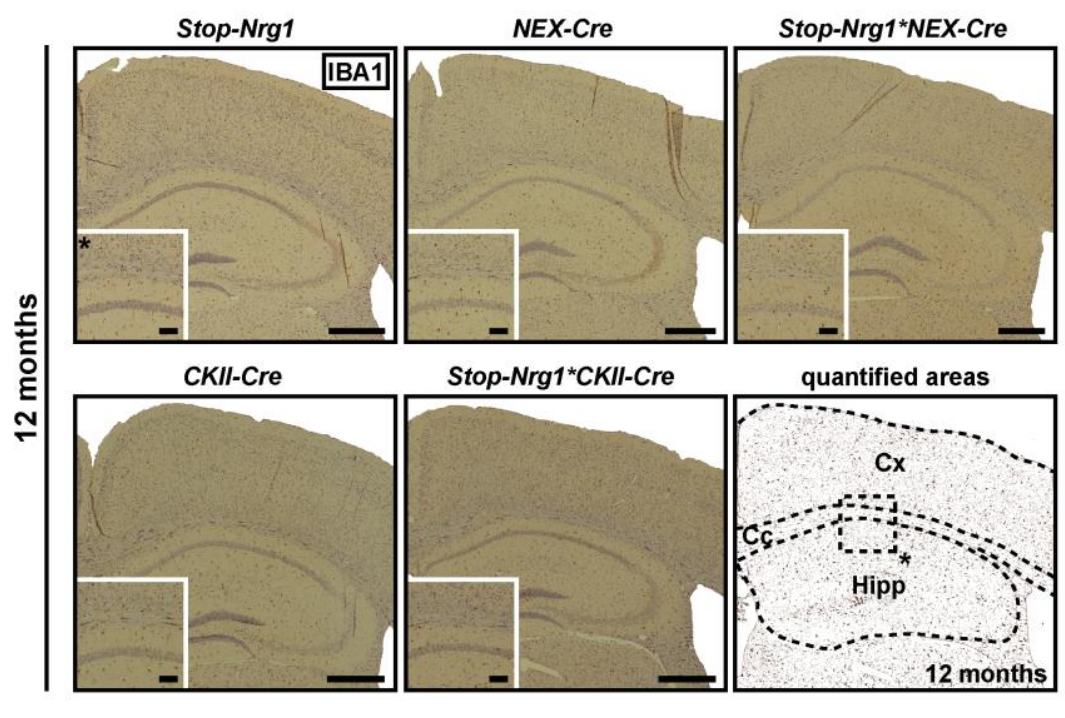

D

Cortex

Corpus callosum

Hippocampus
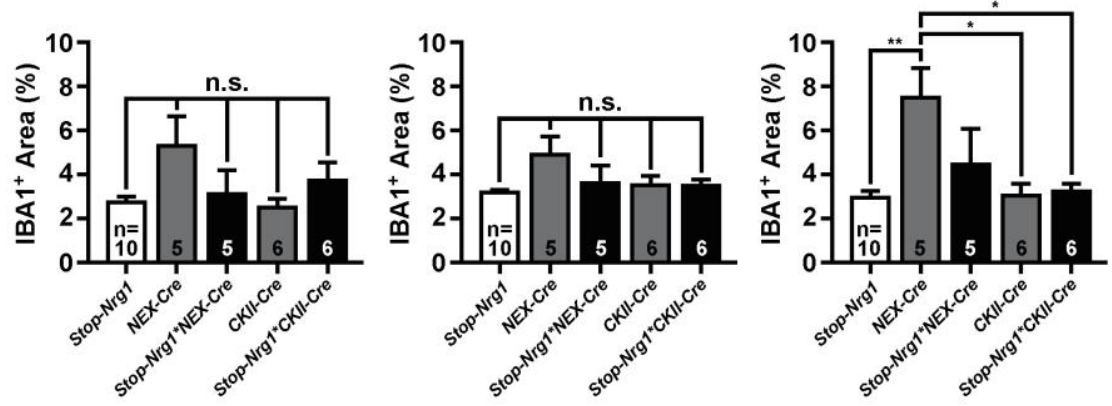
Fig. 19: Stop-Nrg1*NEX-Cre and Stop-Nrg1*CKII-Cre mice show no signs of microgliosis. (A) Chromogenic immunostaining for microglia (IBA1) on coronal paraffin sections from 4 months old Stop-Nrg1*NEX-Cre, Stop-Nrg1 ${ }^{*} \mathrm{CKII}-\mathrm{Cre}$ and control mice (bregma -1.7). Dashed lines mark quantified areas. Dashed square in thresholded black/white image indicates position of high magnification images (Asterisk). Cc, corpus callosum; Cx, cortex; Hipp, hippocampus. Scale bars, $500 \mu \mathrm{m}$ (overvies), $100 \mu \mathrm{m}$ (high magnifications). (B) Semi-automated quantification of IBA $1^{+}$area in cortex, hippocampus and corpus callosum of Stop-Nrg1*NEX-Cre, Stop-Nrg ${ }^{*} \mathrm{CKII-Cre}$ and control mice at 4 months of age reveals absence of microgliosis. (One-way ANOVA; Bonferroni's multiple comparison test; n.s., not significant. n-numbers indicated in the graphs). (C) Chromogenic immunostaining for IBA1 on 1 year old animals as in (A). Scale bars, $500 \mu \mathrm{m}$ (overviews), $100 \mu \mathrm{m}$ (high magnifications). (D) Semi-automated quantification of $\mid \mathrm{BA} 1^{+}$area in 1 year old animals as in (B), reveals significantly increased IBA $1^{+}$area in the hippocampus of $N E X$-Cre mice. Note the moderate increase of $\mathrm{IBA} 1^{+}$area in Stop-Nrg $1^{*} \mathrm{NEX}$-Cre mice, indicating an effect of NEX haploinsufficiency. (One-way ANOVA with Bonferroni's multiple comparison test; n.s., not significant; $n$-numbers indicated in the graphs).

\subsection{Conditional CRD-NRG1 overexpression has no effect on interneuronal migration and maintenance}

NRG1 signaling via ErbB4 has been implicated in the migration of cortical interneurons (Flames et al., 2004). Interneuron numbers and cortical layering are normal in Emx-Cre*Nrg ${ }^{t / f}$ mice (Fig. 8B-E), but mildly altered in Nrg1-tg mice (Fig. 9C, E). In addition, the NRG1-ICD has been shown to influence cell survival by regulating genes associated with apoptosis (Bao et al., 2003).

To determine possible effects of chronic CRD-NRG1 overexpression beginning at embryonic and postnatal stages on the migration and maintenance of cortical neurons, a large-scale quantification of neurons in Stop-Nrg ${ }^{*} N E X-C r e$ and Stop$\mathrm{Nrg} 1{ }^{*} \mathrm{CKII}-\mathrm{Cre}$ mice was conducted. Chromogenic immunostainings were performed on coronal paraffin sections (bregma -1.7) of 4 months and 1 year old StopNrg $1{ }^{*} \mathrm{NEX}$-Cre, Stop-Nrg $1{ }^{*} \mathrm{CKII}-\mathrm{Cre}$, and parental control mice. Brain sections were stained for the pan neuronal marker NeuN and the interneuronal markers GAD67 and parvalbumin and quantified in the cortex and hippocampus.

A semi-automated quantification method was employed for counting $\mathrm{NeuN}^{+}$neuron numbers, based on thresholded images, including watershed-based rendering of positive signals (Fig. 20A, C). Quantification of $\mathrm{NeuN}^{+}$neurons in the cortex of 4 month old Stop-Nrg $1{ }^{\star} \mathrm{NEX}$-Cre and Stop-Nrg1 ${ }^{*} \mathrm{CKII}$-Cre mice showed no differences compared to parental controls, except for a minor difference between StopNrg ${ }^{*} N E X-C r e$ mice and CKII-Cre controls, which harbored significantly less $\mathrm{NeuN}^{+}$ neurons $\left({ }^{*} p<0.05\right)$, however, due to small group size $(n=3)$, this finding requires cautious interpretation (Fig. 20A, B). At 1 year of age NEX-Cre controls and Stop$N r g 1{ }^{*} N E X$-Cre mice showed significantly reduced numbers of $\mathrm{NeuN}^{+}$neurons 
A

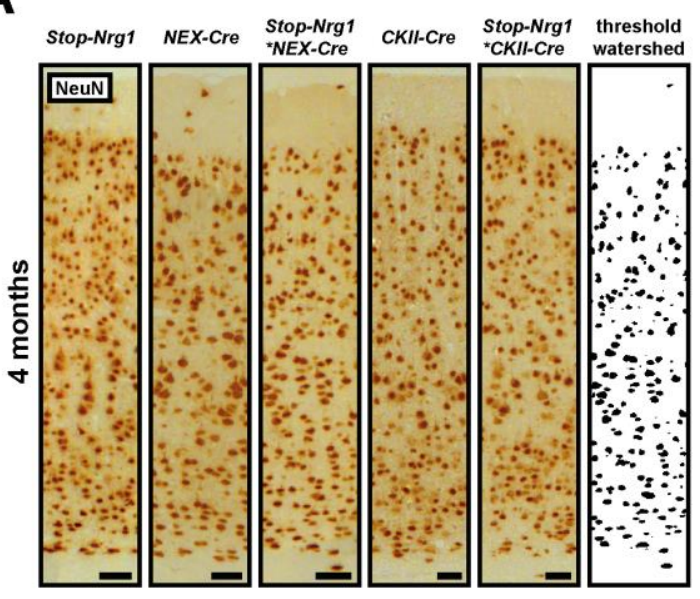

C

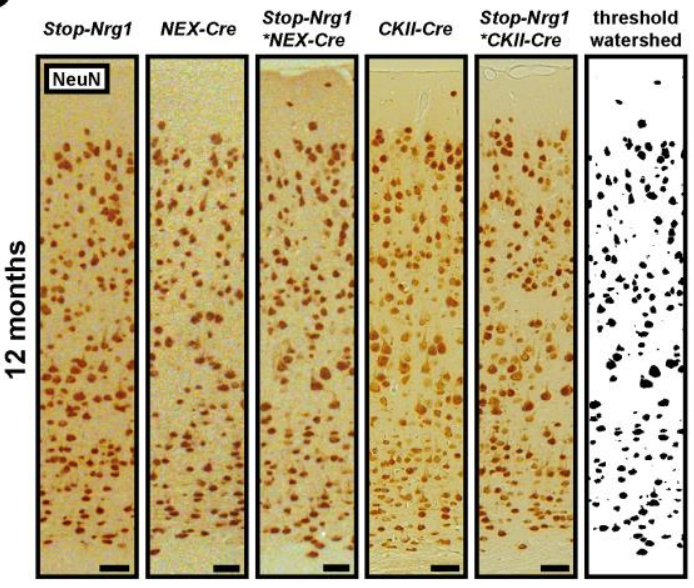

B

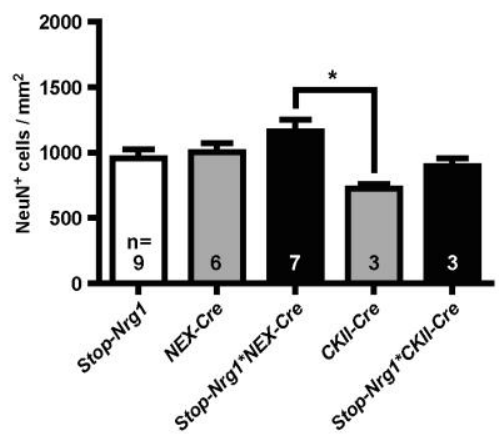

D

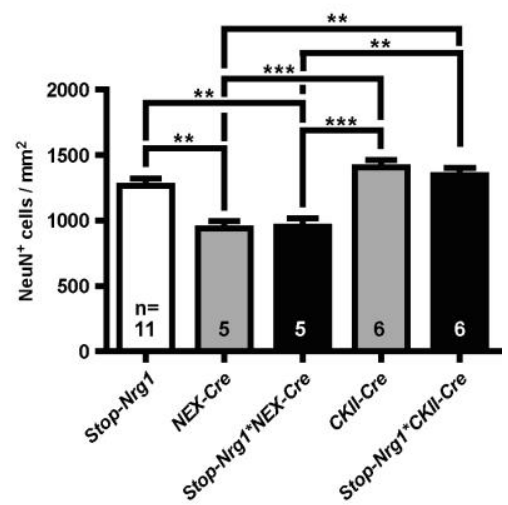

Fig. 20: NEX-Cre and Stop-Nrg1*NEX-Cre mice have reduced numbers of neurons in the cortex at 1 year of age. (A and C) Chromogenic immunostaining for the pan-neuronal marker NeuN on coronal paraffin sections from 4 months and 1 year old Stop-Nrg ${ }^{*} \mathrm{NEX}$-Cre, Stop-Nrg $1^{*} \mathrm{CKII}-\mathrm{Cre}$ and control mice (bregma -1.7), and watershed thresholded images used for semi-autoated quantification of $\mathrm{NeuN}^{+}$neurons. Scale bars, $50 \mu \mathrm{m}$. (B) Semi-automated quantification of $500 \mu \mathrm{m}$ wide columns of the somato-sensory cortex using watershed thresholded images at 4 months of age revealed a slight decrease in neuronal numbers in CKII-Cre mice compared to Stop-Nrg ${ }^{*} N E X$-Cre mice $\left({ }^{*} \mathrm{p}<0.05\right.$, one-way ANOVA; Bonferroni's multiple comparison test; n-numbers indicated in the graph). (D) Semi-automated quantification of $\mathrm{NeuN}^{+}$neurons, as in (B), at 1 year of age revealed a $\sim 20 \%$ reduction of neurons in the cortex of NEX-Cre and Stop-Nrg ${ }^{*} N E X$-Cre mice, indicating an effect of NEX haploinsufficiency $\left({ }^{*} p<0.05,{ }^{* *} p<0.01,{ }^{* * *} p<0.001\right.$; one-way ANOVA; Bonferroni's multiple comparison test; $\mathrm{n}$-numbers indicated in the graph).

compared to other genotypes (Fig. 20C, D). However, this finding emerged from a combination of slightly increased cortical size and reduced numbers of $\mathrm{NeuN}^{+}$cells in mice harboring the NEX-Cre allele (data not shown), resulting in a significant reduction in cell density. Individually, the number of $\mathrm{NeuN}^{+}$cells and cortical size were not significantly different compared to other genotypes. As above, the (modestly) reduced density of $\mathrm{NeuN}^{+}$neurons was associated with NEX haploinsufficiency in NEX-Cre 'knock-in' driver mice, rather than Cre expression per se or HA-NRG1 overexpression, since this effect was not observed in Stop-Nrg1* 
A

A

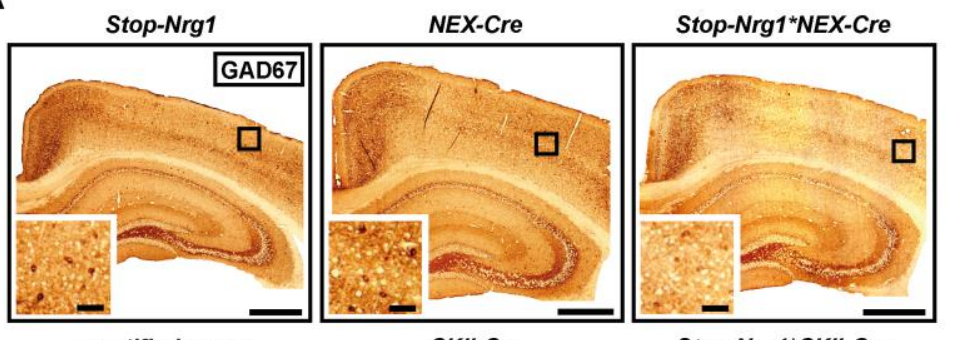

quantified areas

CKII-Cre

Stop-Nrg1 ${ }^{*} \mathrm{CKII}-\mathrm{Cre}$
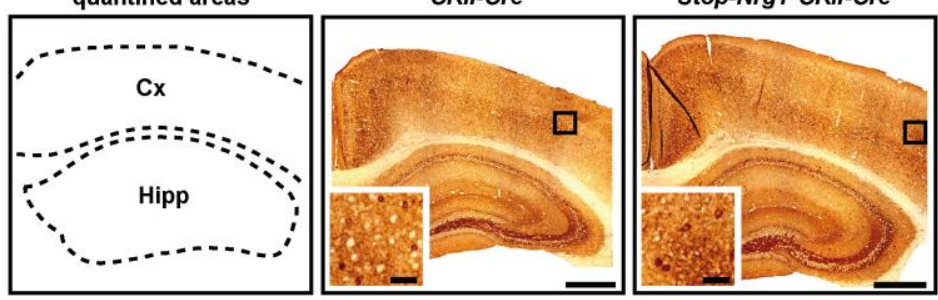

B

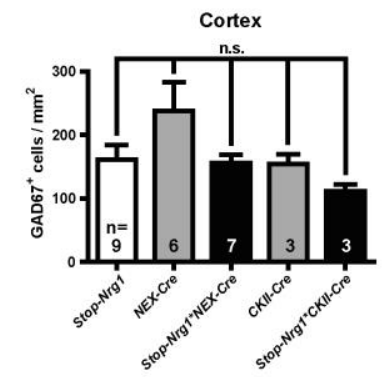

Hippocampus

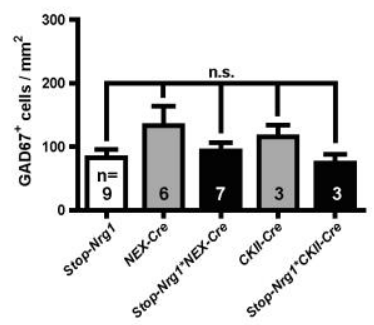

C
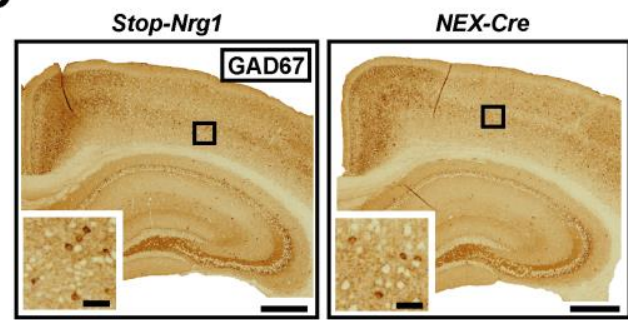

Stop-Nrg1*NEX-Cre
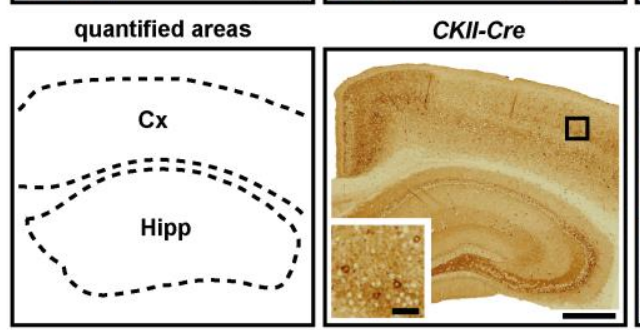

Stop-Nrg1 ${ }^{\star} \mathrm{CK} / \mathrm{I}-\mathrm{Cre}$

D
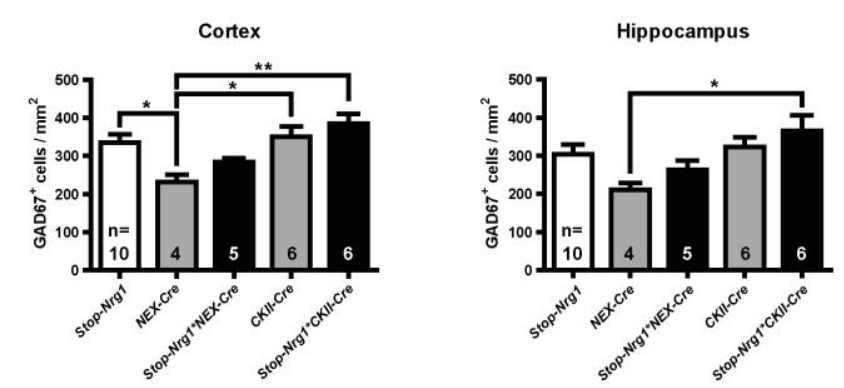

Fig. 21: NEX-Cre mice show reduced numbers of GAD67 ${ }^{+}$interneurons in the cortex and hippocampus at 1 year of age. (A) Chromogenic immunostaining for GAD67 on coronal paraffin sections from 4 months old Stop-Nrg1 ${ }^{\star} \mathrm{NEX}$-Cre, Stop-Nrg1 ${ }^{\star} \mathrm{CK} \mathrm{KI}-\mathrm{Cre}$ and 
control mice (bregma -1.7). Dashed lines indicate quantified areas. Boxes show location of high magnification images. Scale bars, $500 \mu \mathrm{m}$ (overview), $100 \mu \mathrm{m}$ (high magnifications). (B) Quantification of GAD67 $7^{+}$interneurons in the cortex and hippocampus of 4 months old Stop$\mathrm{Nrg} 1{ }^{*} \mathrm{NEX}$-Cre, Stop-Nrg $1{ }^{*} \mathrm{CKII}-\mathrm{Cre}$ and controls showed no significant differences in interneuron numbers (One-way ANOVA; Bonferroni's multiple comparison test; n.s., not significant; $n$-numbers indicated in the graph). ( $C$ and $D) A$ similar analysis of GAD67 ${ }^{+}$ interneurons, as in (A and B), was performed at 1 year of age. (C) Exemplary images of GAD67 immunostainings and quantified areas. Scale bars, $500 \mu \mathrm{m}$ (overview), $100 \mu \mathrm{m}$ (high magnifications). (D) Quantification of GAD67 $7^{+}$cells revealed reduced interneuronal numbers in the cortex and hippocampus of NEX-Cre mice. Stop-Nrg ${ }^{\star} N E X$-Cre mice as well showed a trend to reduced interneuron numbers. $\left({ }^{*} p<0.05,{ }^{* *} p<0.01\right.$; one-way ANOVA with Bonferroni's multiple comparison test; $n$-numbers indicated in the graph).

CKII-Cre or CKII-Cre mice (Fig. 20D). Since the NEX-Cre driver line is a widely used tool in neurobiology, possible neuropathological effects of NEX (NeuroD6) haploinsufficiency must be carefully considered.

Next, interneuronal numbers in the somatosensory cortex and hippocampus (bregma -1.7) were quantified at 4 months and 1 year of age following immunostaining of coronal paraffin sections for GAD67 (Fig. 21A, C, dashed lines mark quantified areas). Counting was performed manually, since GAD67 not only stained cell somata, but also dendritic and axonal arborizations (Fig. 21 A, C, note background staining). Again, the results represent cell density (cells $/ \mathrm{mm}^{2}$ ) to normalize the cell number to the quantified area. No changes in the number of GAD67 ${ }^{+}$interneurons were observed at 4 months of age (Fig. 21A, B). At 1 year of age a significant reduction in the density of $\mathrm{GAD} 67^{+}$interneurons was present in the cortex and hippocampus of NEX-Cre controls compared to other genotypes (Fig. 21D). Similar as above, increased cortical and hippocampal size, without concomitant increase in cell numbers (data not shown), resulted in reduced GAD67 $7^{+}$cell density in NEX-Cre controls. In contrast, embryonic (Stop-Nrg $1{ }^{\star} \mathrm{NEX}$-Cre) or postnatal (Stop-Nrg $1{ }^{\star} \mathrm{CKII-}$ Cre) HA-NRG1 overexpression showed no major effect on interneuron density in the cortex or hippocampus (Fig. 21D).

$\mathrm{PV}^{+}$interneurons present the largest group of ErbB4 expressing interneurons (Neddens and Buonanno, 2009; Vullhorst et al., 2009; Fazzari et al., 2010). To specifically examine these cells, coronal paraffin sections were immunostained for parvalbumin. Similar to the GAD67 analysis, interneuron numbers were manually counted in the hippocampus and cortex (bregma -1.7) of Stop-Nrg1*NEX-Cre, Stop$\mathrm{Nrg} 1{ }^{*} \mathrm{CKII}-\mathrm{Cre}$, and parental control mice at 4 months and 1 year of age (Fig. 22A, C, dashed lines indicate quantified areas). At 4 months no significant differences in the number of $\mathrm{PV}^{+}$interneurons were observed in the cortex and hippocampus of Stop$\mathrm{Nrg} 1{ }^{*} \mathrm{NEX}$-Cre or Stop-Nrg ${ }^{*} \mathrm{CKII}$-Cre mice compared to controls (Fig. 22A, B). 
A

Stop-Nrg1

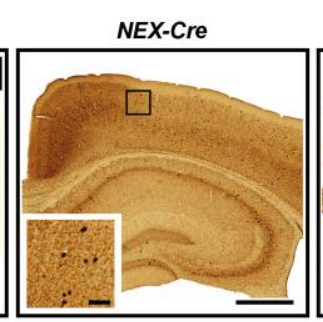

Stop-Nrg1*NEX-Cre

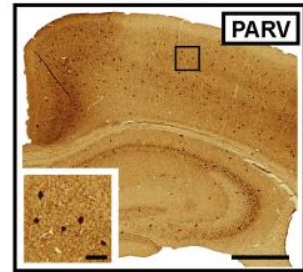

CKII-Cre
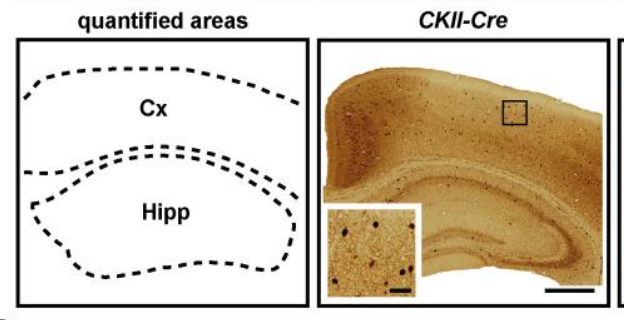

B
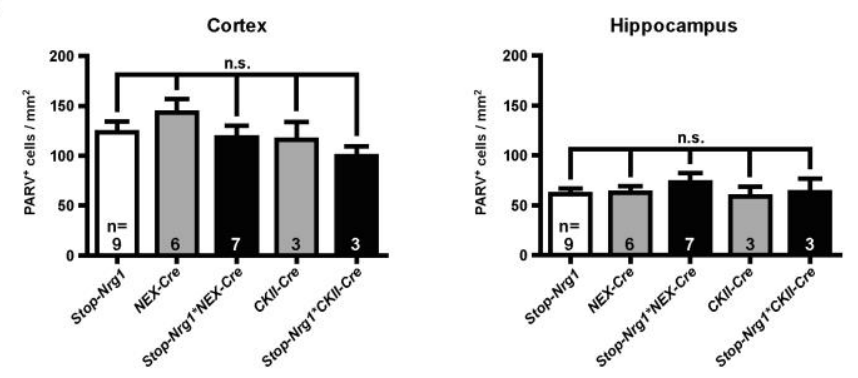

C
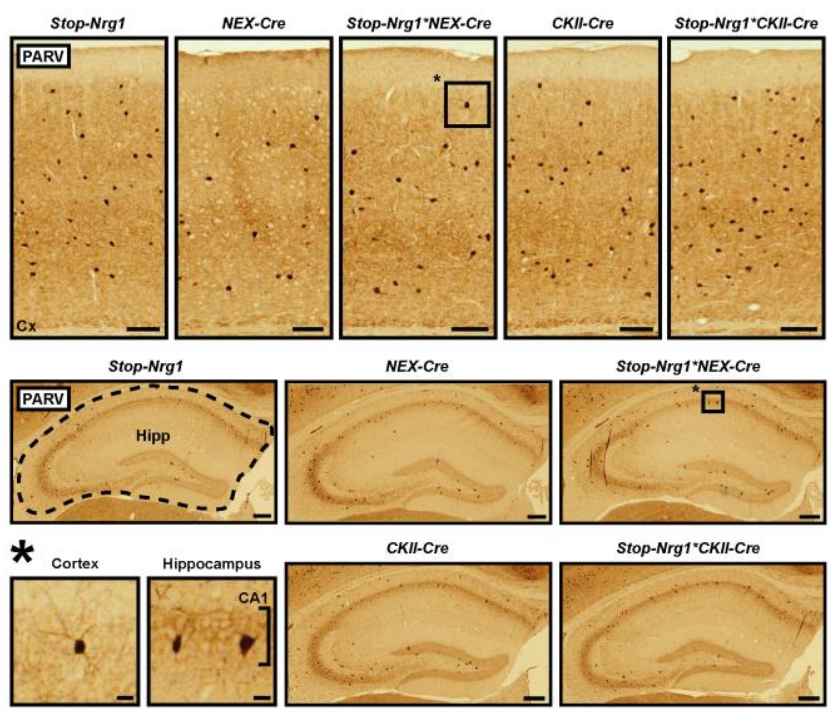

D

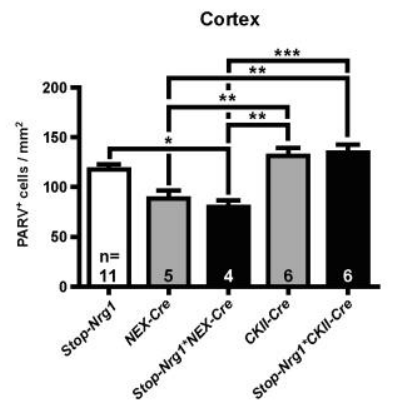

Hippocampus

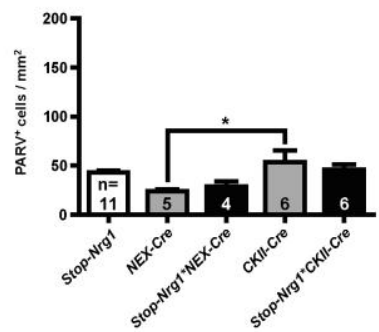

Fig. 22: NEX-Cre and Stop-Nrg1*NEX-Cre mice have reduced numbers of $\mathrm{PV}^{+}$ interneurons in the cortex and hippocampus at one year of age. (A) Chromogenic immunostainings for PV on coronal paraffin sections from 4 months old Stop-Nrg1 ${ }^{\star} N E X-C r e$, 
Stop-Nrg $1^{*} \mathrm{CKII}-\mathrm{Cre}$ and control mice (bregma -1.7). Dashed lines indicate quantified areas. Boxes highlight positions of high magnification images. Scale bars, $500 \mu \mathrm{m}$ (overview), 100 $\mu \mathrm{m}$ (high magnifications). (B) Quantification of $\mathrm{PV}^{+}$interneurons in the cortex and hippocampus at 4 months of age showed no difference between genotypes. (One-way ANOVA; Bonferroni's multiple comparison test; n.s., not significant; $n$-numbers indicated in the graph). (C) Similar chromogenic immunostaining for PV as in (A) for 1 year old mice. Dashed line indicates the area quantified in the hippocampus. Boxes indicate positions of high magnification images shown in the lower panel (Asterisk). Scale bars, $500 \mu \mathrm{m}$ (overview), $100 \mu \mathrm{m}$ (high magnification). (D) Quantification of $\mathrm{PV}^{+}$interneurons at 1 year of age in the cortex and hippocampus of NEX-Cre mice revealed reduced numbers of $\mathrm{PV}^{+}$ interneurons. A similar effect in the cortex and a trend in the hippocampus were also observed in Stop-Nrg ${ }^{*} N E X$-Cre mice, arguing for an effect of NEX haploinsufficiency. Note that in the cortex of 1 year old mice only a $500 \mu \mathrm{m}$ wide column of the somato-sensory cortex was quantified. ( ${ }^{*} p<0.05,{ }^{* *} p<0.01,{ }^{* *} p<0.001$; one-way ANOVA with Bonferroni's multiple comparison test; $n$-numbers indicated in the graph).

At 1 year of age a significantly reduced density of $\mathrm{PV}^{+}$interneurons was detected in NEX-Cre controls and Stop-Nrg1*NEX-Cre mice (Fig. 22C, D). As above, a combination of increased cortical and hippocampal sizes and mildly reduced numbers of $\mathrm{PV}^{+}$interneurons (data not shown) resulted in a significant reduction of cell density. Again, this effect was associated with NEX haploinsufficiency, as it was also present in Stop-Nrg1 ${ }^{*}$ NEX-Cre mice, but not CamKII-Cre driver mice (Fig. 22D). Taken together, HA-NRG1 overexpression in cortical projection neurons (with early embryonic or postnatal onset) caused no major abnormalities in cortical interneuron migration and/or maintenance.

At 1 year of age a significantly reduced density of $\mathrm{PV}^{+}$interneurons was detected in NEX-Cre controls and Stop-Nrg $1{ }^{*} N E X$-Cre mice (Fig. 22C, D). As above, a combination of increased cortical and hippocampal sizes and mildly reduced numbers of $\mathrm{PV}^{+}$interneurons (data not shown) resulted in a significant reduction of cell density. Again, this effect was associated with NEX haploinsufficiency, as it was also present in Stop-Nrg1*NEX-Cre mice, but not CamKII-Cre driver mice (Fig. 22D). Taken together, HA-NRG1 overexpression in cortical projection neurons (with early embryonic or postnatal onset) caused no major abnormalities in cortical interneuron migration and/or maintenance.

\subsection{Cortical-restricted HA-NRG1 overexpression is not linked to ventricular enlargement observed in Thy1.2 promoter-driven transgenic mice}

Ventricular enlargement is a robust and well replicated feature in SZ and Nrg1-tg mice with cortical and subcortical HA-NRG1 overexpression show enlarged lateral ventricles (Agarwal et al., 2014). 
A
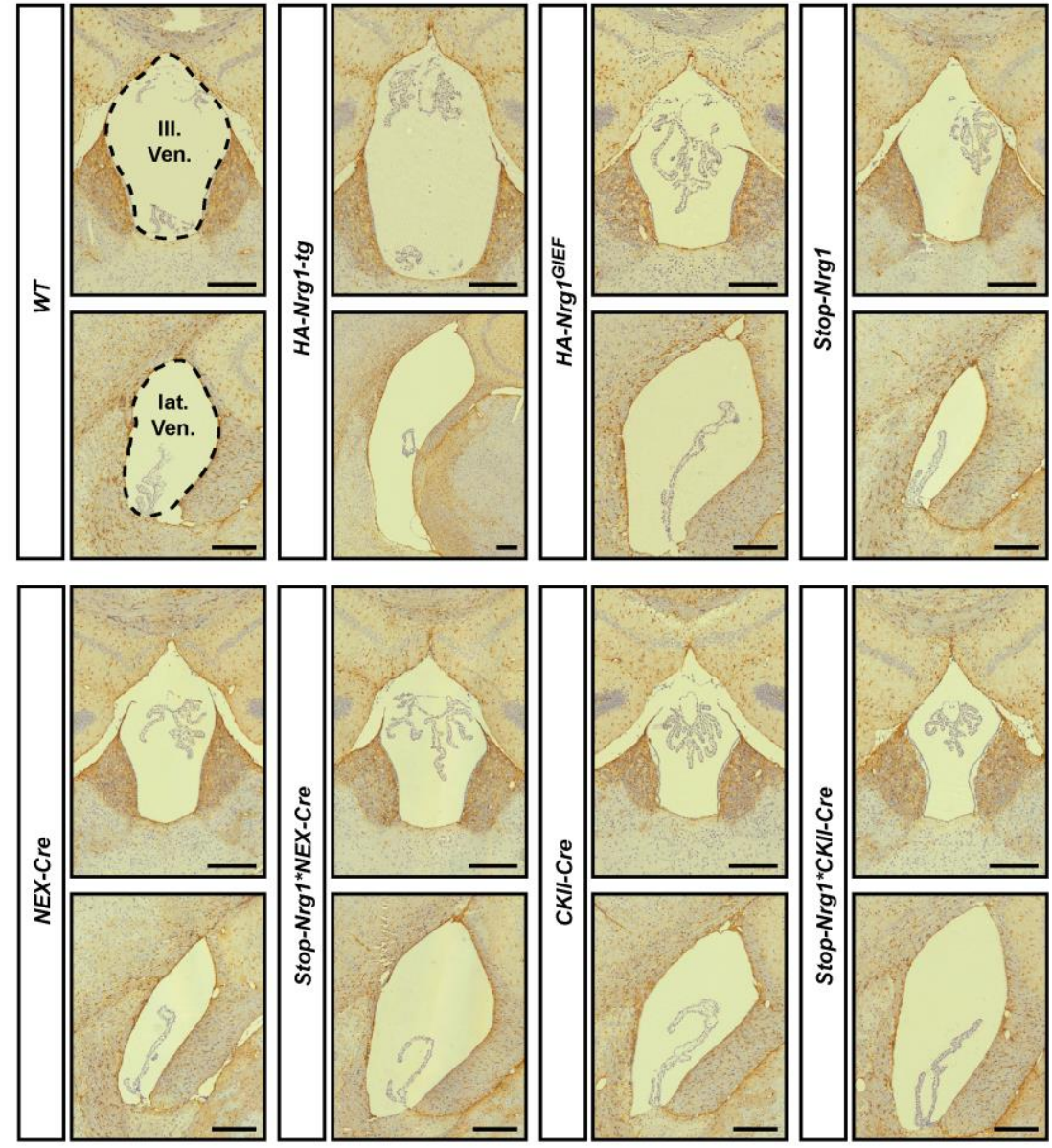

B

third ventricle

lateral ventricles (avg. I+r)
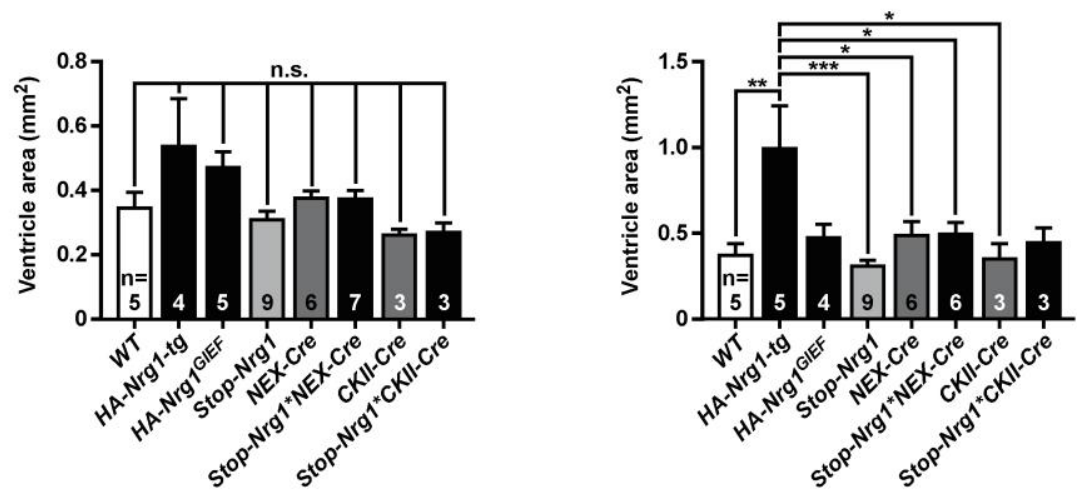

Fig. 23: Lateral ventricles are enlarged in HA-Nrg1-tg, but not in $H A-N r g 1^{\text {GIEF }}$ or conditional HA-NRG1 overexpressing mice. (A) Representative images of third and lateral ventricles of Thy1.2 promoter-driven and conditional HA-Nrg1 transgenic mouse lines and controls. Chromogenic immunostaining for GFAP on coronal paraffin sections at bregma -1.7. Scale bars, $250 \mu \mathrm{m}$. (B) Because ventricles are three dimensional structures with changing cross section profile, ventricle sizes of Thy 1.2 promoter-driven $\mathrm{HA}-\mathrm{Nrg} 1$-tg and $\mathrm{HA}-\mathrm{Nrg} 1^{\text {GIEF }}$, as well as conditional Stop-Nrg ${ }^{*} \mathrm{NEX}$-Cre, Stop-Nrg $1^{*} \mathrm{CKII}-\mathrm{Cre}$ mice and controls were measured and averaged in at least three coronal paraffin sections per mouse, to increase the reliability of the analysis (bregma -1.7). HA-Nrg1-tg mice have enlarged lateral ventricle sizes and show a tendency to enlarged third ventricles. $\left({ }^{*} p<0.05,{ }^{* *} p<0.01,{ }^{* * *} p<0.001\right.$; n.s., not significant; one-way ANOVA with Bonferroni's multiple comparison test; $\mathrm{n}$-numbers indicated in the graph). 
To systematically compare ventricular sizes in different NRG1 transgenic mouse models, sizes of third and lateral ventricles were determined in Thy1.2-driven $H A$ Nrg1-tg and HA-Nrg $1^{\text {GIEF }}$ mice, as well as in conditional Stop-Nrg1*NEX-Cre and Stop-Nrg $1{ }^{*} \mathrm{CKII}$-Cre mice. HA-Nrg $1^{\text {GIEF }}$ mice express an HA epitope-tagged variant of CRD-NRG1, which mimicks proteolytic cleavage in the juxtamembrane stalk region by BASE-1, therefore lacks the CTF, and ends with the amino acid sequence: "GIEF" for (Glycine-Isoleucine-Glutamic Acid (E)-Phenylalanine (F)). Similar to HA-Nrg1-tg mice, these mice show hypermyelination of the CNS and PNS and express this NRG1 variant at high levels in neurons of the brain (Velanac et al., 2011). Ventricular size was determined on coronal paraffin sections (bregma -1.7). Representative images are shown in Fig. 23A, the dashed lines indicate measured areas.

As expected, $\mathrm{HA}-\mathrm{Nrg} 1$-tg mice showed enlarged ventricles, similar to Nrg1-tg mice (Agarwal et al., 2014), however the enlargement of the third ventricle was not significant, when compared to the other genotypes (Fig. 23B). Analysis of lateral ventricles (average of left and right lateral ventricle area) revealed that ventricle size in HA-Nrg1-tg mice was almost doubled compared to all other genotypes, however this result was not significant when compared to $\mathrm{HA}-\mathrm{Nrg} 1^{\text {GIEF }}$ and Stop-Nrg $1^{*} \mathrm{CKII}$ Cre mice (Fig. 23B).

It appears that subcortical expression of full length CRD-NRG1 is required for ventricular enlargement. However, expression of $\mathrm{HA}-\mathrm{NRG} 1^{\mathrm{GIEF}}$ at high levels in subcortical regions does not affect ventricular sizes, indicating that the NRG1 CTF might be involved in the development of enlarged ventricles.

\subsection{Thy1.2-driven NRG1 transgenic mice have reduced body weight}

Intracerebroventricular injections of recombinant NRG1 cause decreased body weight in hamsters (Snodgrass-Belt et al., 2005). Furthermore, intraperitoneal administration of recombinant NRG1 has recently been shown to trigger decreased food intake and results in reduced weight gain in normal-weight mice, by affecting leptin levels (Ennequin et al., 2015). When handling our transgenic mice, we observed that Nrg1-tg and HA-Nrg1-tg mice were always leaner than their WT littermates. To analyze this observation in more detail, the body weight of adult males from several NRG1 overexpressing transgenic mouse lines at an age of 12 to 18 weeks was determined. In line with previous observations, Thy1.2-driven Nrg1-tg and $H A-N r g 1$-tg mice were significantly leaner than controls and Stop-Nrg1*NEX-Cre mice, but not than CKII-Cre and Stop-Nrg $1{ }^{*} \mathrm{CKII}$-Cre mice. In fact Stop-Nrg $1^{\star} \mathrm{CKII-}$ Cre males were significantly leaner than Stop-Nrg1*NEX-Cre males (Fig. 24), 
suggesting that subcortical overexpression of CRD-NRG1, for instance in thalamic and hypothalamic regions, is involved in the weight reduction, as Stop- $\mathrm{Nrg} 1^{\star} \mathrm{CKII}-\mathrm{Cre}$ mice show more subcortical expression than Stop-Nrg1*NEX-Cre mice (compare Fig. 14, 16).

\section{Body weight of 12-18 weeks old males}

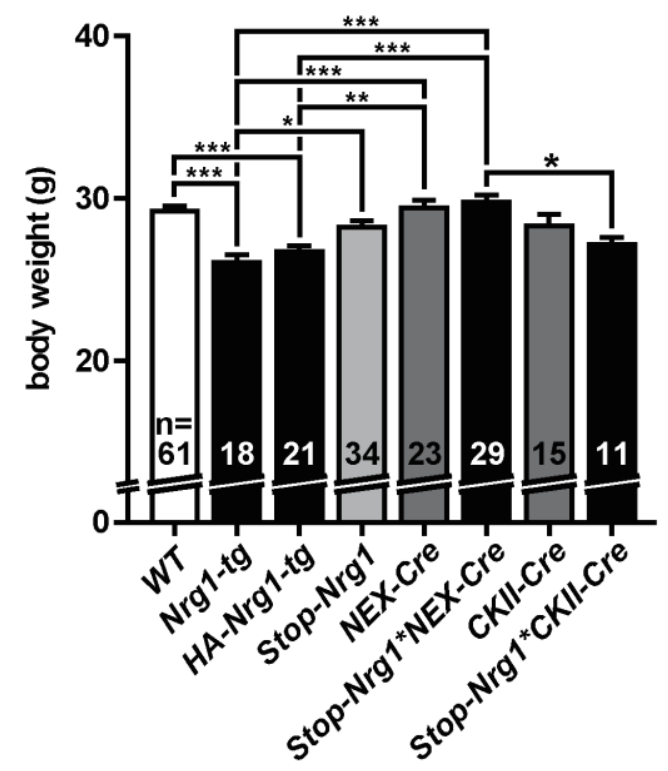

Fig. 24: 'Global' Nrg1-tg and HA-Nrg1-tg mice have reduced body weight. Body weight of NRG1 transgenic mouse models was determined in male mice at an age of 12 to 18 weeks. Thy1.2 promoter-driven $\mathrm{Nrg} 1$-tg and $\mathrm{HA}-\mathrm{Nrg} 1$-tg mice have significantly lower body weight than controls and conditional Stop-Nrg1 ${ }^{*} \mathrm{NEX}$-Cre mice. CKII-Cre and Stop-Nrg1 ${ }^{*} \mathrm{CKII}-\mathrm{Cre}$ were not significantly heavier than Thy 1.2 promoter-driven models. Stop-Nrg $1{ }^{*} \mathrm{CKII}-\mathrm{Cre}$ mice were significantly leaner than Stop-Nrg1 ${ }^{*} N E X$-Cre mice. $\left({ }^{*} p<0.05,{ }^{* *} p<0.01,{ }^{* * *} p<0.001\right.$; one-way ANOVA with Bonferroni's multiple comparison test; $n$-numbers indicated in the graph).

\subsection{Stop-Nrg1 ${ }^{\star} N E X-C r e$ mice show no signs of anxiety-like behavior, decreased PPI or cognitive impairments, but exhibit hyperactivity}

Overexpression of full length HA-CRD-NRG1 in HA-Nrg1-tg mice causes behavioral changes, such as anxiety-like behavior in the open field test (Fig. 10E) and PPI deficits (Fig. 10D). In these mice, pan-neuronal CRD-NRG1 overexpression in the brain starts at around E16 and persists at high levels throughout life. In StopNrg1*NEX-Cre mice moderate HA-CRD-NRG1 overexpression is restricted to projection neurons of the neocortex, hippocampus and amygdala, starting at E12.

To test whether cortical restricted HA-NRG1 overexpression leads to abnormal behavior, adult (age 8-25 weeks) Stop-Nrg1*NEX-Cre mice $(\mathrm{n}=20)$ and controls, including WT $(n=15)$, Stop-Nrg1 $(n=11)$ and NEX-Cre mice $(n=22)$, were examined in a battery of behavioral tests. To assess locomotor activity and anxiety, 
A

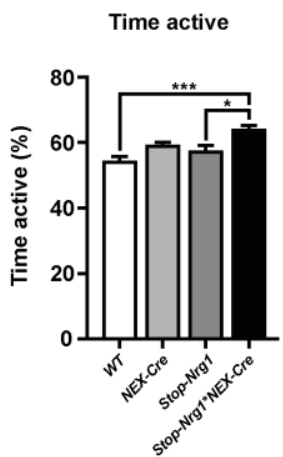

B

Exploration time

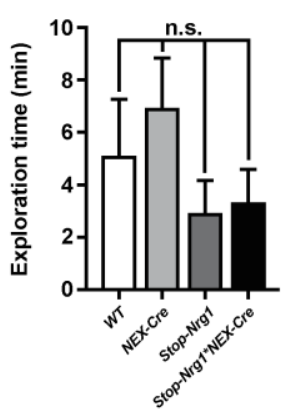

C

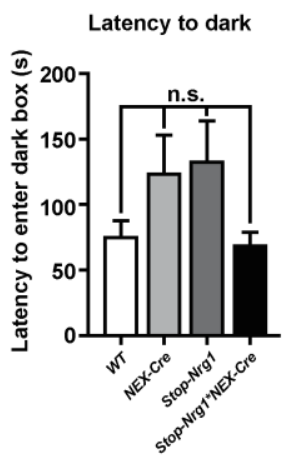

D

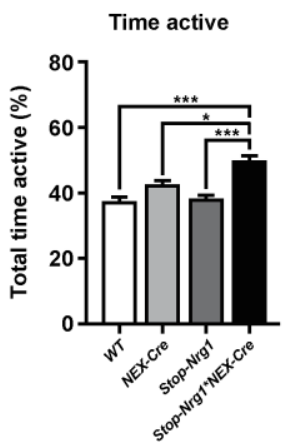

Distance travelled

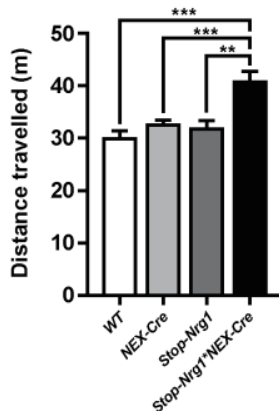

Distance travelled

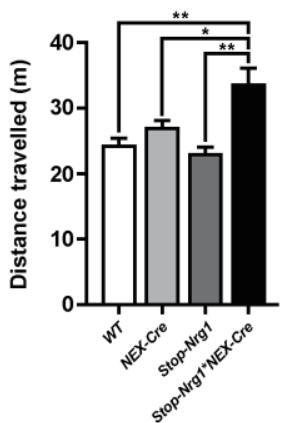

Number of crossings
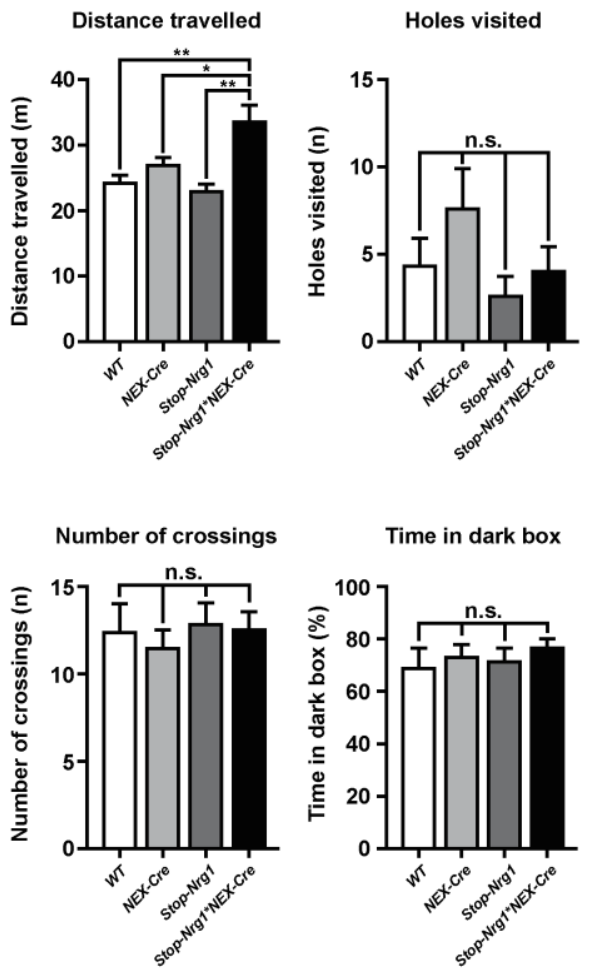

Distance travelled in $1 \mathrm{~min}$ intervals
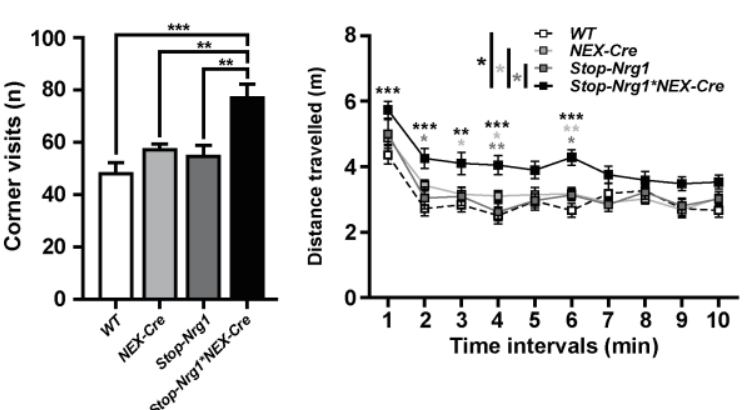

Distance travelled in 1 min intervals
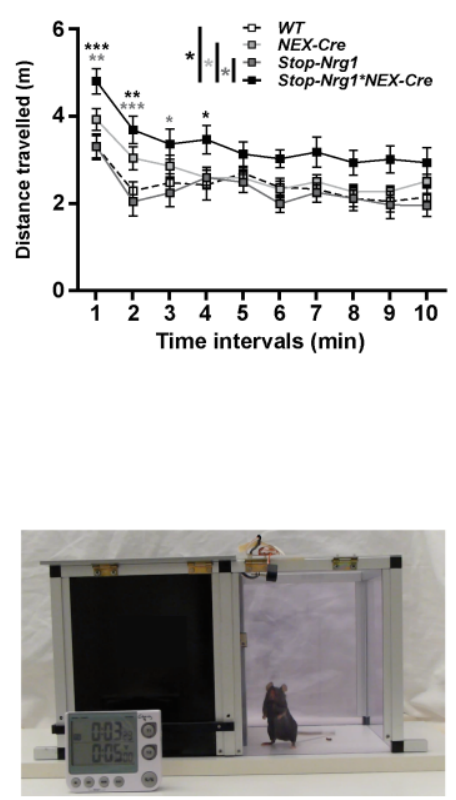

Fig. 25: Stop-Nrg1*NEX-Cre mice exhibit novelty-induced hyperactivity and increased fighting behavior in the tail suspension test. (A) Stop-Nrg $1^{*} N E X$-Cre mice are hyperactive in the open field test compared to parental controls and show increased time active, distance travelled and more corner visits. Distance travelled plotted in 1 min intervals over the length of the experiment reveals novelty-induced hyperactivity. No signs of altered anxiety behavior were observed. (B) Stop-Nrg1*NEX-Cre males are hyperactive in the hole board test, but show similar exploration times and hole visits as controls. Distance travelled plotted as $1 \mathrm{~min}$ intervals confirms novelty-induced hyperactivity. (C) Stop-Nrg1*NEX-Cre mice show normal behavior in the light-dark preference test compared to controls. No signs of altered anxiety 
behavior were observed. (right) Image of the experimental setup. (D) Stop-Nrg1*NEX-Cre mice exhibit increased fighting behavior in the first, second and fifth minute of the tail suspension test compared to controls. NEX-Cre mice fought more than WT in the first and second minute and more than Stop-Nrg1 mice in the second minute of the test. (right) Image of a mouse during the experiment. Male mice were tested at an age of 8-25 weeks. Stop$N r g 1{ }^{*} N E X$-Cre mice $(n=20), W T(n=15)$, Stop-Nrg1 $(n=11), N E X-C r e(n=22) .\left({ }^{*} p<0.05\right.$, ${ }^{* *} p<0.01,{ }^{* *} p<0.001$; n.s., not significant; one-way ANOVA with Bonferroni's multiple comparison test).

mice were tested in the open field test. While signs for increased anxiety-like behavior were not observed (data not shown), Stop-Nrg1 ${ }^{\star} N E X$-Cre mice exhibited hyperactivity. Stop-Nrg1*NEX-Cre mice were more active ( 10\% increase) compared to WT and Stop-Nrg1 mice, but not to NEX-Cre mice (Fig. 25A). They travelled longer distances ( $30 \%$ increase) and visited more corners ( $44 \%$ increase) compared to all control groups (Fig. 25A). When the distance travelled was plotted as 1 minute intervals over the 10 minutes duration of the experiment, Stop-Nrg1*NEXCre mice travelled more distance over the entire course of the experiment (Fig. 25A). This effect was highly significant in the first half of the experiment, when compared to WT controls and significant in most of the 1 minute intervals compared to the two parental control strains (Stop-Nrg1 and NEX-Cre), suggesting novelty induced hyperactivity.

To assess the exploratory drive and anxiety related behavior under different conditions, mice were tested in the hole board test. In this test mice explore an open arena equipped with holes, which trigger exploratory behavior, i.e. nose poking. While Stop-Nrg1*NEX-Cre mice showed no changes in exploratory behavior (Fig. $25 \mathrm{~B})$, they travelled longer distances ( $35 \%)$. When plotted as 1 minute intervals, Stop-Nrg ${ }^{*} N E X$-Cre mice travelled longer distances during the first half of the test and showed higher activity during the entire experiment compared to controls, similar to the hyperactivity phenotype observed in the open field test (Fig. 25B). To examine anxiety-related behavior more specifically, the light-dark preference test was employed. This test measures the animal's preference for dark, enclosed places over bright, exposed places. Mice that did not enter the dark compartment within 6 minutes were excluded from the test (two WT and two NEX-Cre mice). Similar to open field and hole board tests, Stop-Nrg1*NEX-Cre mice showed no signs of increased anxiety (Fig. 25C).

Next, the tail suspension test was employed, which was developed to assess mood levels and as a screening test for potential antidepressant drugs. In this test the mice are hung by the tail for 6 minutes and thereupon try to escape this aversive situation (Fig. 25D). Longer periods of immobility are considered as depressive-like behavior. When tested, Stop-Nrg ${ }^{\star} N E X$-Cre mice spent more time trying to avoid the 
aversive situation compared to WT and Stop-Nrg1 mice $\left({ }^{* *} \mathrm{p}<0.001\right)$ and NEX-Cre controls ( ${ }^{*} p<0.05$ ), thus displaying decreased depressive-like behavior (Fig. 25D). When plotted over time, Stop-Nrg1 ${ }^{*} N E X$-Cre mice showed more activity than WT in the first, second, and fifth minute of the test $\left({ }^{* *} p<0.001\right)$, in minute 2 more activity than Stop-Nrg1 $\left.{ }^{* * *} p<0.001\right)$ and in minute 5 more activity than NEX-Crecontrols $\left({ }^{* * *} p<0.001\right)$ (Fig. 25D). However, NEX-Cre controls also struggled more than WT mice in the first $\left({ }^{* *} p<0.01\right)$ and second minute of the test $\left({ }^{*} p<0.05\right)$, and in the second minute NEX-Crecontrols showed more activity than Stop-Nrg1 controls $\left({ }^{* *} \mathrm{p}\right.$ $<$ 0.001) (Fig. 25D). This finding again supports the hypothesis that haploinsufficiency in NEX-Cre mice could affect brain functions, including behavior.

NRG1/ErbB4 signaling is involved in both short-term and long-term neural plasticity (Li et al., 2007; Woo et al., 2007), CRD-NRG1 overexpression results in impaired LTP (Agarwal et al., 2014). In addition, HA-Nrg1-tg mice showed deficits in working memory (Wehr et al., in preparation). Ablation of NRG1 in projection neurons, using the CKII-Cre driver line caused reduction in contextual and cued fear memory. Together, these findings suggest that NRG1 plays a role in learning, memory and other cognitive processes.

To test the influences of cortical restricted HA-NRG1 overexpression on hippocampus-dependent learning and memory, Stop-Nrg1*NEX-Cre mice were tested in contextual and cued fear conditioning. When mice were placed into the shocking chamber for 2 min on the first day, they showed normal baseline behavior (normal exploration with no freezing).

Subsequently, mice were subjected to two pairings of the conditioned stimulus (CS, a tone of $80 \mathrm{db}$, for $30 \mathrm{sec}$ ), followed by the aversive unconditioned stimulus (US, mild electric foot shock, $0.4 \mathrm{~mA}$ for $2 \mathrm{sec}$ ) separated by $30 \mathrm{sec}$. Reaction to both the CS and US was comparable in all groups. 24 hours later, the retention of contextual fear conditioning was assessed by placing mice back into the fear conditioning context box for $2 \mathrm{~min}$. Contextual fear response was scored by measuring the freezing response (steady suppression of locomotor or exploratory activity). All genotype groups showed comparable freezing behavior as a consequence of exposure to the context, however Stop-Nrg1 ${ }^{*} N E X$-Cre mice showed a tendency to reduced freezing response (Fig. 26D). 24 hours later mice were placed in a novel context box with no similarities to the shocking chamber, and scored for freezing behavior in response to the new context for 2 min. All genotypes showed similar freezing responses to the new context that were slightly increased over the context baseline, but significantly lower than responses to the context (Fig. 26D). 
A

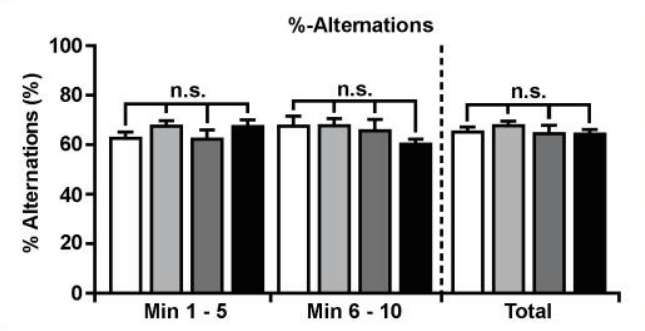

C

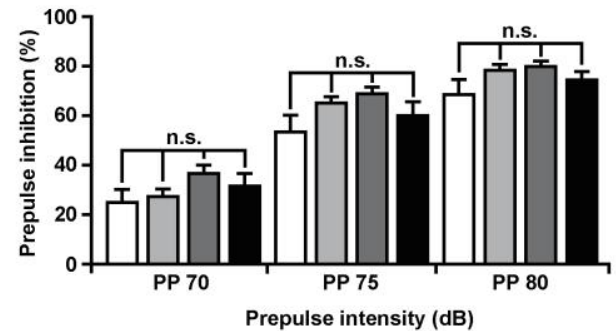

D

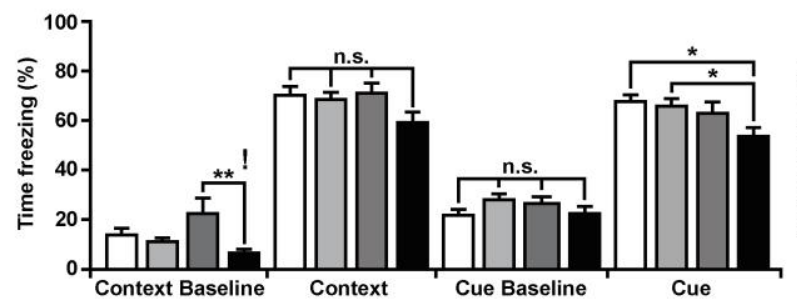

$\mathbf{F}$

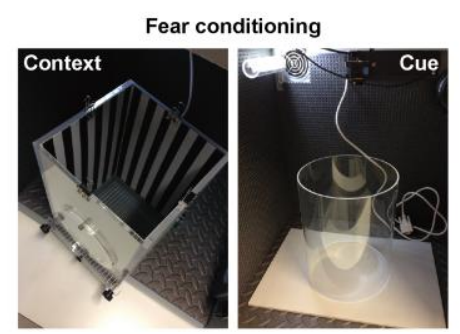

G

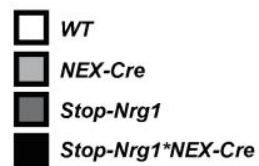

B
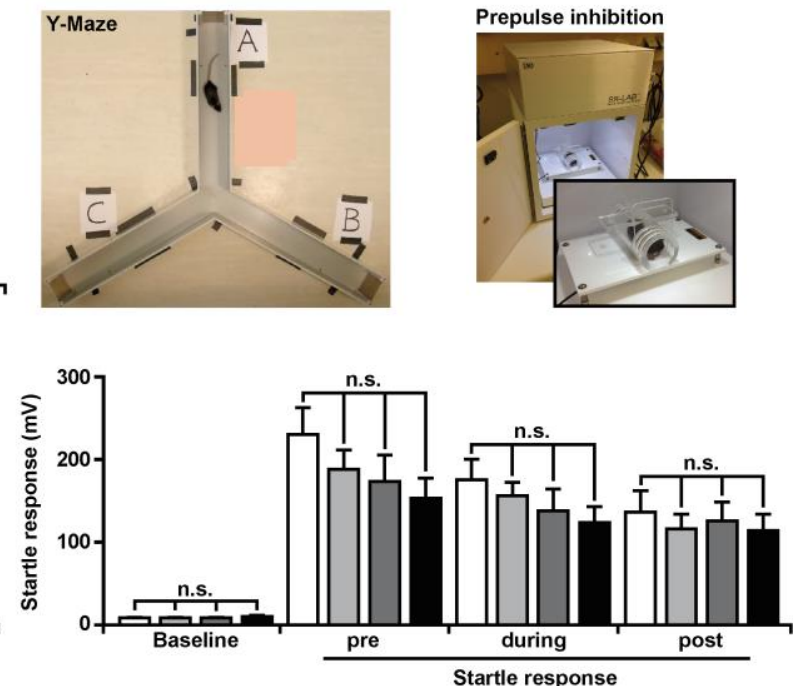

E
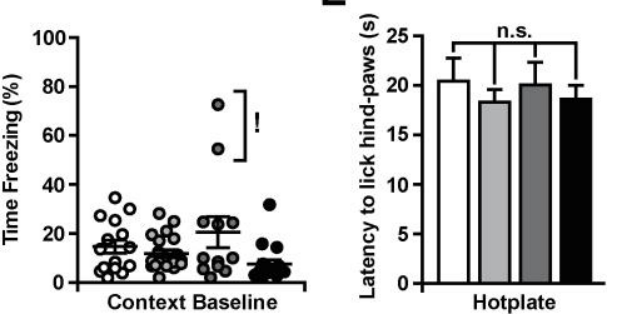

Fig. 26: Stop-Nrg1 ${ }^{*} \mathrm{NEX}$-Cre mice show no impairments in working memory and sensorimotor gating, but exhibit reduced cued fear memory. (A) Stop-Nrg $1^{*} N E X$-Cre mice show no impairments of working memory in the first and second half, as well as the total $\%$ alternations compared to controls in the $\mathrm{Y}$-maze spontaneous alternation test. (right) Image of the experimental setup. (B) Image of the SR-Lab startle response system used for the PPI test. Mice were placed into restraining tubes on a deflection sensitive platform. (C) (left) Stop$\mathrm{Nrg} 1^{*} \mathrm{NEX}$-Cre mice show no differences in the percentage of prepulse inhibition at prepulses of 70,75 and $80 \mathrm{~dB}$ and no differences in the startle responses prior, during and after the conditioning (right). (D) Stop-Nrg ${ }^{*} N E X$-Cre mice display reduced cued fear memory compared to WT and NEX-Cre controls. Stop-Nrg1 mice show increased context baseline freezing behavior due to two outliers (exclamation mark), illustrated in the scatter plot (right). Since these two mice performed normal in all other tasks, they were kept in the analysis. (E) Normal pain sensitivity in all genotypes in the hotplate test. (F) Ugo Basile fear conditioning system. The striped box on the left was used for contextual, and the clear cylinder on the right as novel context for cued fear memory. (G) Genotype color-code of the bargraphs. Male mice were tested at an age of 8-25 weeks. Stop-Nrg $1^{*} N E X$-Cre mice $(n=20), W T(n=15)$, Stop$\operatorname{Nrg} 1(n=11), N E X-C r e(n=22) .\left({ }^{*} p<0.05,{ }^{* *} p<0.01,{ }^{* * *} p<0.001\right.$; $n . s .$, not significant; oneway ANOVA with Bonferroni's multiple comparison test).

Next, mice were exposed for 2 min to the auditory CS (tone of $80 \mathrm{db}$ ), and while 
control groups (WT, Stop-Nrg1 and NEX-Cre) showed similar freezing responses, Stop-Nrg1*NEX-Cre mice showed a significant reduction in freezing responses compared to $W T$ and NEX-Cre controls, but not to Stop-Nrg1 controls (Fig. 26D). Impaired fear response in Stop-Nrg ${ }^{*} N E X$-Cre mice could not be caused by a deficit in detecting auditory stimuli as these mice performed well in the prepulse inhibition (PPI) test (Fig. 26C).

To investigate the effect of cortical HA-CRD-NRG1 overexpression on working memory, Stop-Nrg1*NEX-Cre mice were tested in the Y-maze spontaneous alternation test. Mice were allowed to explore the maze for $10 \mathrm{~min}$ and the number of choices and the percentage of alternations were scored. No difference was observed between Stop-Nrg1*NEX-cre mice and controls in the number of choices (data not shown) and in the percentage of alternations (Fig. 26A). Thus, Stop-Nrg1 ${ }^{*} N E X$-Cre mice showed no deficits in working memory in the Y-maze, in contrast to HA-Nrg1-tg mice,

Finally, assessment in the hotplate test (Fig. 26E) and sensorimotor gating by employing the PPI test, showed no changes in pain perception as well as normal startle responses and no difference in PPI in Stop-Nrg1*NEX-Cre mice (Fig. 26C), in contrast to HA-Nrg1-tg mice (Fig. 10, Agarwal et al., 2014).

\subsection{Postnatal cortical overexpression of HA-NRG1 in Stop-Nrg $1^{\star} \mathrm{CKII-}$ Cre mice has only minor effects on behavior}

SZ is considered a developmental disease with first symptoms occurring in early adulthood (Sham et al., 1994). It was shown in previous studies that deletion of NRG1 from projection neurons starting at P5 in CKII-Cre*Nrg $1^{f / f}$ mice resulted in hypoactivity in the open field test in 3 months old mice and impaired contextual and cued fear conditioning in 12 months old mice (Agarwal et al., 2014). Stop-Nrg1*NEXCre mice showed absence of anxiety-like behavior and no PPI impairment (Fig. 10D, E), but hyperactivity in the open field and hole board test, and increased fighting behavior in the tail suspension test (Fig. 25A, B, D).

To distinguish embryonic from postnatal effects of NRG1 overexpression on behavioral functions, a cohort of Stop-Nrg1*CKII-Cre mice $(n=7)$ and parental controls, Stop-Nrg1 $(n=19)$, and CKII-Cre $(n=11)$ was tested at 3 to 6 months of age in the same battery of behavioral tests as before. First, locomotor activity and anxiety was assessed in the open field test. Here, only minor hyperactivity was observed for the distance travelled and the number of corner visits in Stop-Nrg $1^{\star} \mathrm{CKII}$-Cre mice 
A

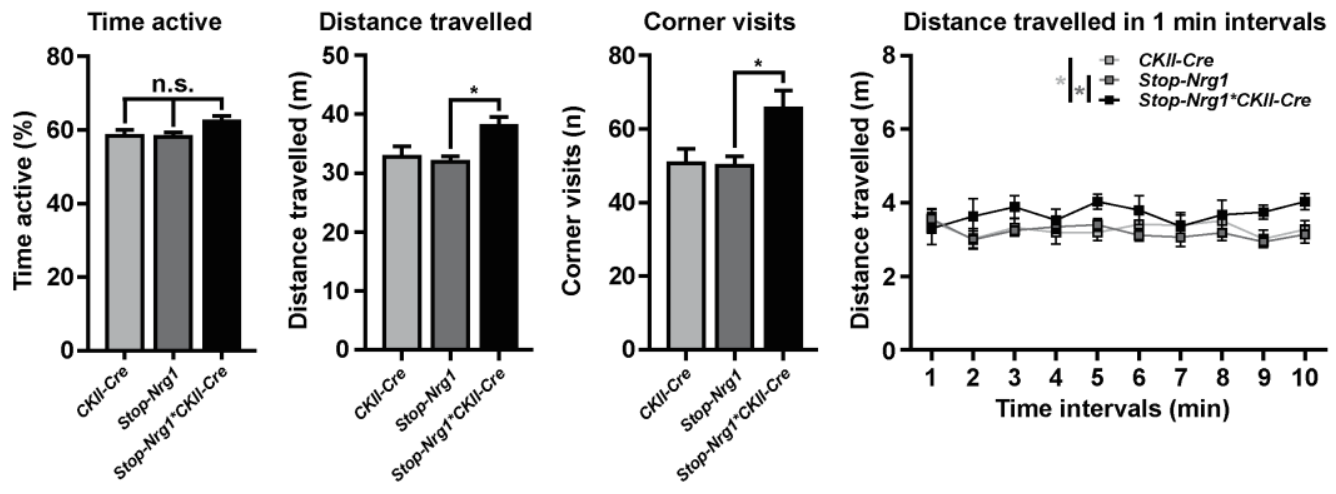

B
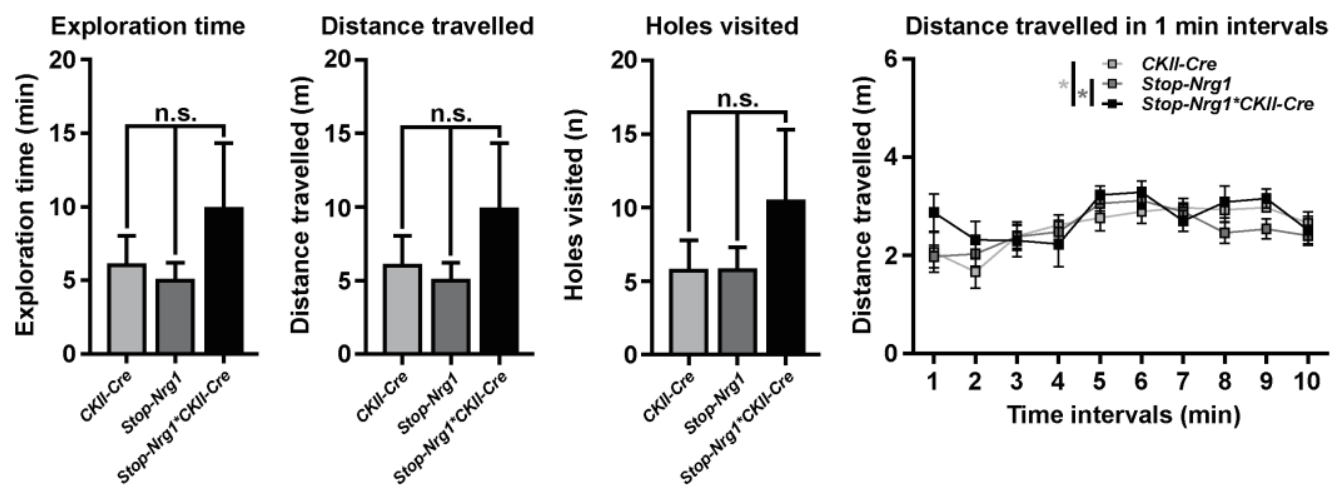

C
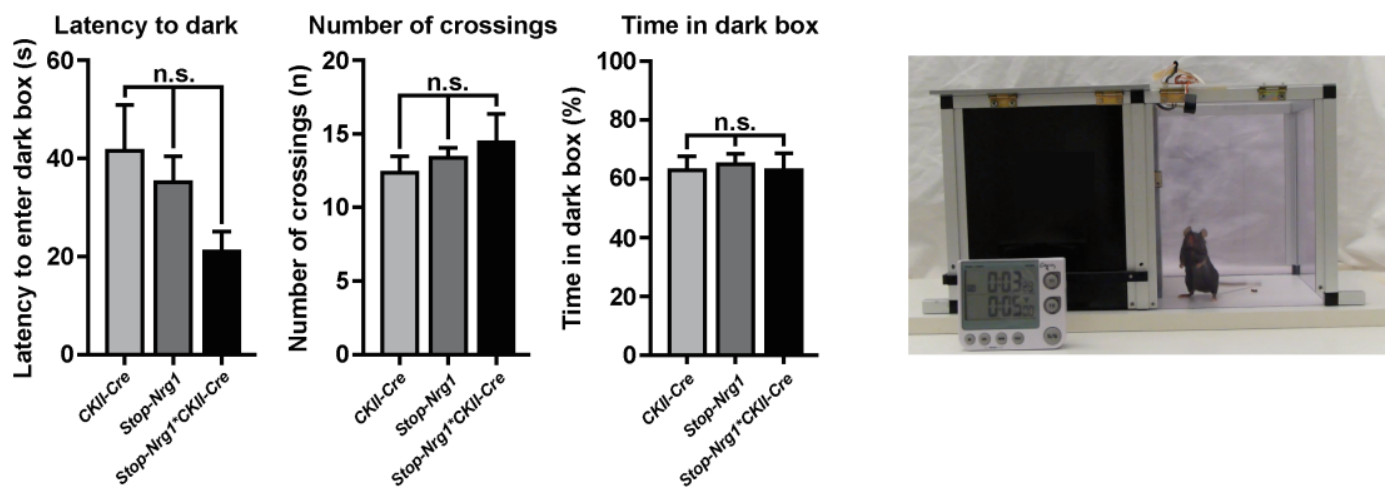

D
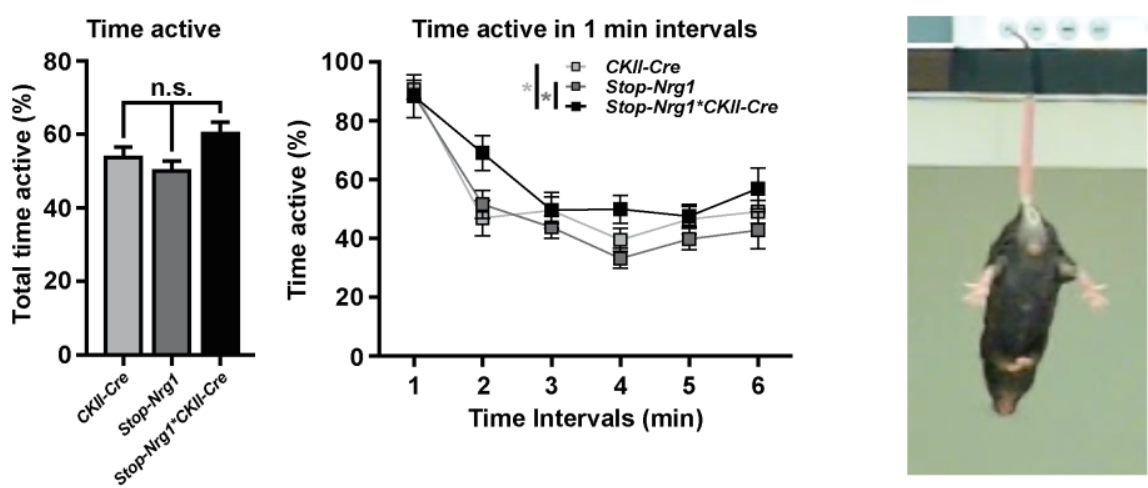

Fig. 27: Postnatal cortical overexpression of HA-NRG1 in Stop-Nrg1 ${ }^{\star} \mathrm{CKII-Cre} \mathrm{mice} \mathrm{has}$ only minor effects on activity. (A) Stop-Nrg1 ${ }^{*} \mathrm{CKII-Cre} \mathrm{mice} \mathrm{show} \mathrm{increased} \mathrm{distance}$ travelled and corner visits in the open field test compared to Stop-Nrg1, but not to CKII-Cre controls. Distance travelled plotted as $1 \mathrm{~min}$ intervals revealed no novelty-induced 
hyperactivity, as Stop-Nrg $1{ }^{*} \mathrm{CKII}$-Cre mice show only a minor increase of distance travelled. (B) Normal behavior of Stop-Nrg1 ${ }^{*} \mathrm{CKII}-\mathrm{Cre}$ mice in the hole board test. Only minor increases in the exploration time, distance travelled and hole visits were observed. When plotted as 1 min intervals, no difference between genotypes was detected for the distance travelled. (C) Stop-Nrg $1{ }^{*} \mathrm{CKII}-\mathrm{Cre}$ mice show normal behavior in the light-dark preference test. (right) Image of the experimental setup. (D) Only minor increase of fighting behavior in Stop$\mathrm{Nrg} 1{ }^{*} \mathrm{CKII}-\mathrm{Cre}$ mice in the tail suspension test. Plotting the percentage of time active as $1 \mathrm{~min}$ intervals reveals only minor increases of activity in the second, fourth and last minute of the experiment. (right) Image of a mouse during the experiment. Male mice were tested at 3 to 6 months of age. Stop-Nrg ${ }^{*} \mathrm{CKII}-\mathrm{Cre}(\mathrm{n}=7)$, Stop-Nrg1 $(\mathrm{n}=19)$, CKII-Cre $(\mathrm{n}=11) .\left({ }^{*} \mathrm{p}<0.05\right.$; n.s., not significant; one-way ANOVA with Bonferroni's multiple comparison test).

compared to Stop-Nrg1 controls, but not to CKII-Cre controls (Fig. 27A). Similarly, Stop-Nrg $1{ }^{*} \mathrm{CK}$ II-Cre mice showed only tendencies of hyperactivity and increased exploratory behavior in the hole board test, but none of these tendencies was significant compared to parental controls (Fig. 27B). To more specifically test anxietyassociated behavior, the light-dark preference test was performed. Both, the number of crossings and the time spend in one or the other compartment was unaltered in these mice (Fig. 27C), indicating normal exploratory behavior and anxiety levels in Stop-Nrg $1{ }^{*} \mathrm{CKII}$-Cre mice. Finally, Stop-Nrg $1{ }^{*} \mathrm{CKII}$-Cre mice showed a nonsignificant increase in overall time active in the tail suspension test (Fig. 27D). Thus, Stop-Nrg $1{ }^{*} \mathrm{CKII}$-Cre mice showed no signs of depressive-like states, but also no increase in motivation to escape the aversive situation. In summary, Stop-Nrg ${ }^{*} \mathrm{CKII}-$ Cre mice do not replicate the hyperactivity phenotype observed in Stop-Nrg1 ${ }^{\star} N E X$ Cre mice.

To assess hippocampus-dependent learning and memory, Stop-Nrg $1{ }^{*} \mathrm{CKII}$-Cre mice were tested in contextual and cued fear conditioning. Following the same experimental procedure as above, Stop-Nrg $1{ }^{*} \mathrm{CKII}-\mathrm{Cre}$ mice showed no deficits in contextual and cued fear memory (Fig. 28D).

When tested also Stop-Nrg $1{ }^{*} \mathrm{CKII}-\mathrm{Cre}$ mice and controls in the $\mathrm{Y}$-maze spontaneous alternation test, Stop-Nrg $1{ }^{*} \mathrm{CKII}-\mathrm{Cre}$ mice showed normal exploration behavior, and there was no significant difference between Stop-Nrg $1^{*} \mathrm{CKII}-\mathrm{Cre}$ mice and controls in the number of choices (data not shown) and the percentage of alternations (Fig. 28A). Thus Stop-Nrg $1^{*} \mathrm{CKII}$-Cre mice showed no impairments of working memory in this test.

Finally Stop-Nrg $1{ }^{*} \mathrm{CKII}-\mathrm{Cre}$ mice and controls showed similar response to pain stimuli in the hotplate test (Fig. 28E), and no differences were observed in startle response and PPI (Fig. 28C).

In summary, postnatal conditional overexpression of the same HA-CRD-NRG1 variant as in HA-Nrg1-tg and Stop-Nrg1 ${ }^{*} \mathrm{NEX}$-Cre mice shows only minor effects on behavioral functions in Stop-Nrg ${ }^{*} \mathrm{CKII}-\mathrm{Cre}$ mice. 
A

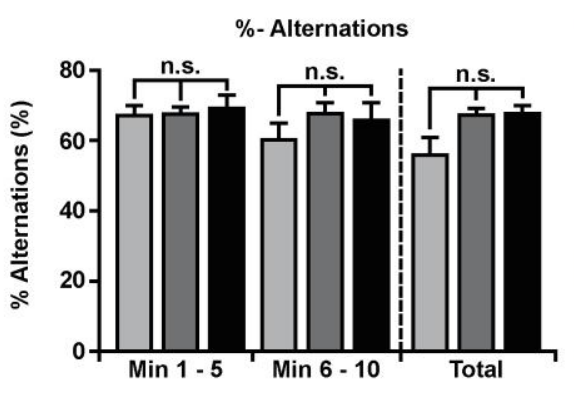

C

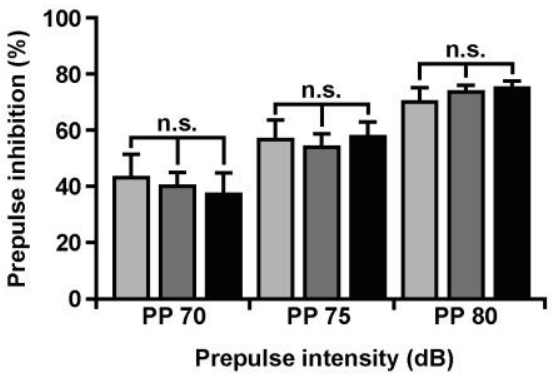

D

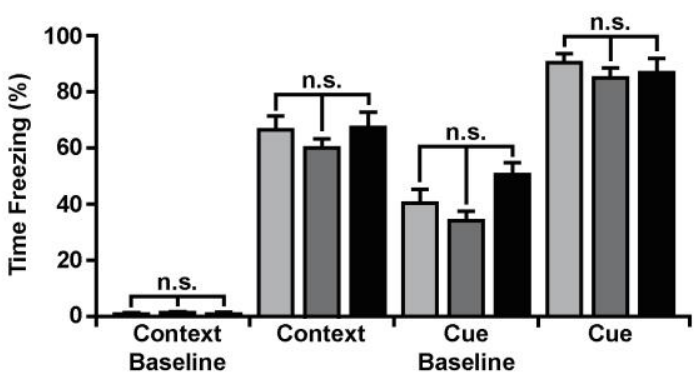

E

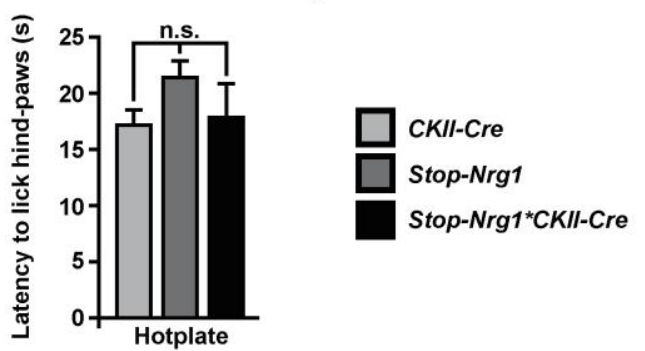

$\mathbf{F}$
B

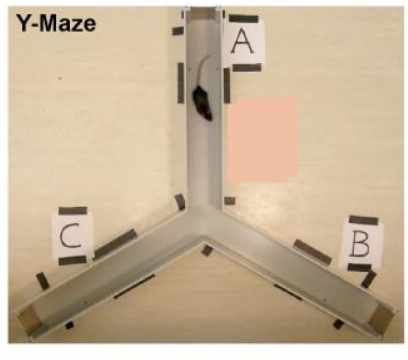

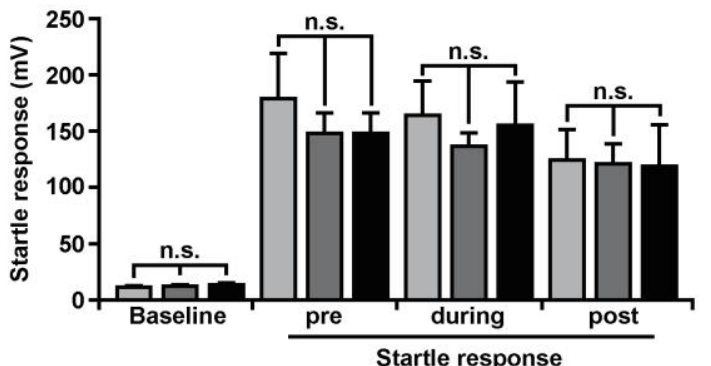

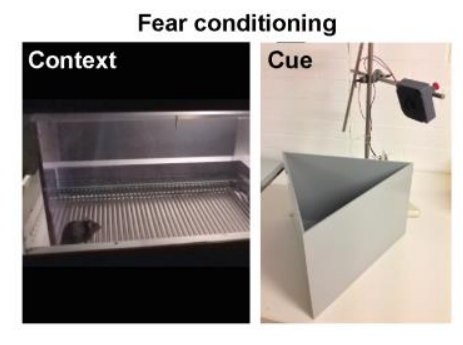

Fig. 28: Stop-Nrg1*CKII-Cre mice exhibit normal learning and memory as well as sensorimotor gating. (A) Stop-Nrg $1{ }^{\star} \mathrm{CK}$ II-Cre mice show no impairments of working memory in the Y-maze spontaneous alternation test compared to parental controls. (right) Image of the experimental setup. (B) Image of the SR-Lab startle response system used for the PPI test. Mice were placed into restraining tubes on a deflection sensitive platform. (C) (left) Stop-Nrg $1{ }^{*} \mathrm{CKII}-\mathrm{Cre}$ mice show no differences in the percentage of prepulse inhibition at prepulses of 70,75 and $80 \mathrm{~dB}$ and no differences in the startle responses prior, during and after the conditioning (right). (D) No impairments of learning and memory in Stop-Nrg $1{ }^{*} \mathrm{CKII-}$ Cre mice in contextual and cued fear memory compared to controls. (right) TSE Systems fear conditioning setup. The box on the left was used for contextual fear conditioning and the triangular box on the right as a novel context for cued fear memory. (E) Normal pain sensitivity in all genotypes in the hotplate test. (F) Genotype color-code of the bargraphs. Male mice were tested at 3 to 6 months of age. Stop-Nrg $1{ }^{*}$ CKII-Cre $(\mathrm{n}=7)$, Stop-Nrg1 $(\mathrm{n}=$ 19), CKII-Cre ( $n=11)$. ( ${ }^{*} p<0.05$; n.s., not significant; one-way ANOVA with Bonferroni's multiple comparison test). 


\subsection{Stop-Nrg1 ${ }^{\star} N E X-C r e E R T 2$ mice as a tool for acute overexpression of NRG1 in the adult mouse brain}

Acute changes in NRG1/ErbB4 signaling may provide better insight into 'normal' NRG1 functions in the brain. To model acute overexpression of HA-CRD-NRG1 in adult mice, we employed the NEX-CreERT2 driver mouse line (Agarwal et al., 2011). This mouse line allows tamoxifen induced, Cre-mediated recombination. Tamoxifen is a synthetic estrogen receptor (ER) ligand, which induces the dissociation of cytosolic CreERT2 from HSP90, nuclear import of CreERT2, and site-specific recombination of loxP-flanked target genes.

To test the efficiency of different tamoxifen concentrations to induce Crerecombination in the brain, we crossbred NEX-CreERT2 mice to R26R-floxtdTomato reporter mice (Madisen et al., 2010). Double transgenic mice were injected intraperitoneally (IP) for 2 or 10 days with increasing tamoxifen concentrations, ranging from 25-100 mg/kg body weight. Tamoxifen injections at $100 \mathrm{mg} / \mathrm{kg}$ body weight for 10 days were defined as 'full induction' protocol. Four weeks after the last injection, mice were perfused with $4 \%$ PFA and coronal vibratome sections of the brain were prepared. Mice injected for 2 days with tamoxifen at $25 \mathrm{mg} / \mathrm{kg}$ body weight showed only few recombined cells in the cortex, the hippocampal layers CA1 and CA3, and the amygdala (Fig. 29A). Mice injected for 2 days with tamoxifen at 50 $\mathrm{mg} / \mathrm{kg}$ body weight showed an increased number of recombined cells in these areas, but still displayed a single cell recombination pattern (Fig. 29A). Tamoxifen injection for 2 days at $100 \mathrm{mg} / \mathrm{kg}$ resembled full induction, yet there were still markedly less cells recombined than after 10 days tamoxifen injection at $100 \mathrm{mg} / \mathrm{kg}$ body weight. (Fig. 29A). In summary, protocol 1 and 2 (25-50 mg/kg body weight of tamoxifen for 2 days) appeared suitable for single cell genetics.

For biochemical verification of tamoxifen-induced HA-NRG1 expression, StopNrg ${ }^{*} N E X-C r e E R T 2$ and Stop-Nrg1 control mice were injected for 10 days with tamoxifen (100 mg/kg body weight) and brain tissue was collected 1 month later. Western blot analysis of protein lysates from hippocampus and thalamus with an antibody directed against the C-terminus of NRG1 confirmed NRG1 overexpression in the hippocampus, but not thalamus (Fig. 29A, B), in line with absence of thalamic recombination in NEX-CreERT2 mice. However, NRG1 overexpression levels in Stop-Nrg ${ }^{*} N E X-C r e E R T 2$ mice did not match the levels of chronic overexpression in Stop-Nrg1*NEX-Cre mice (Fig. 29, B). In a similar experiment Stop-Nrg1*NEXCreERT2 and control mice were injected for 10 days with tamoxifen $(100 \mathrm{mg} / \mathrm{kg}$ body weight) and analyzed by immunostaining 1 month later. Fluorescent immunostaining 
A

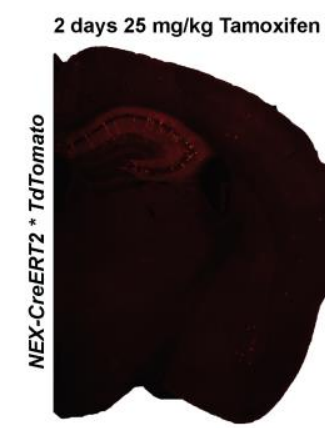

B

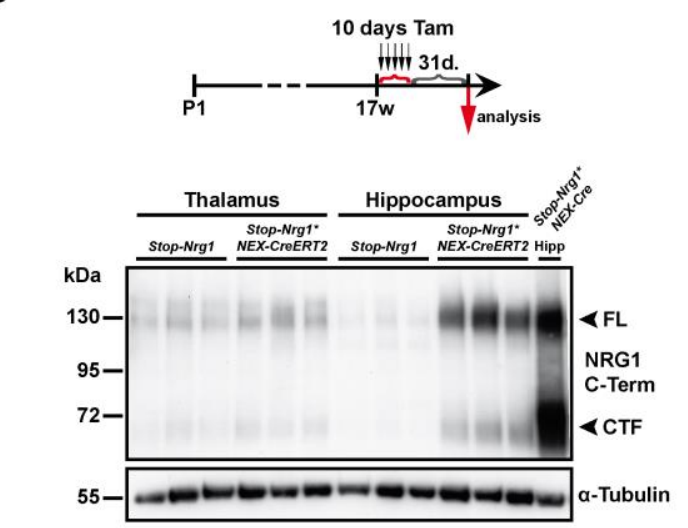

D

Stop-Nrg1^TdTomato ${ }^{\star} N E X-C r e E R T 2$
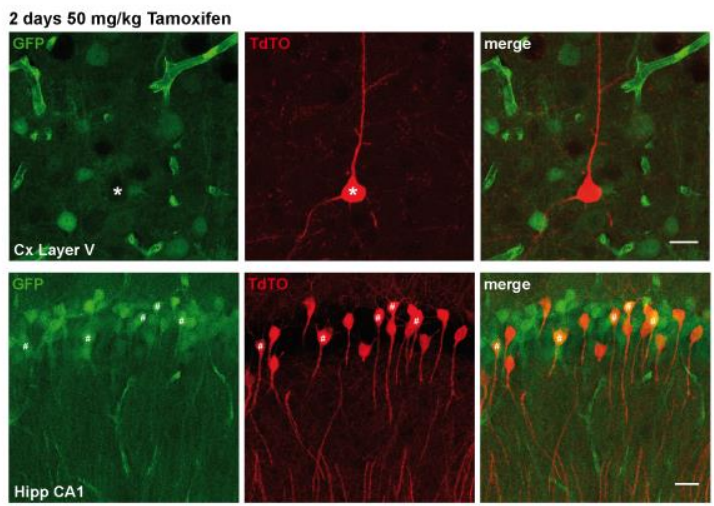

2 days $50 \mathrm{mg} / \mathrm{kg}$ Tamoxifen

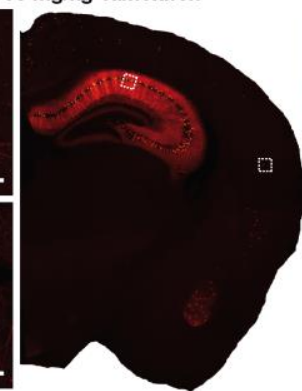

C
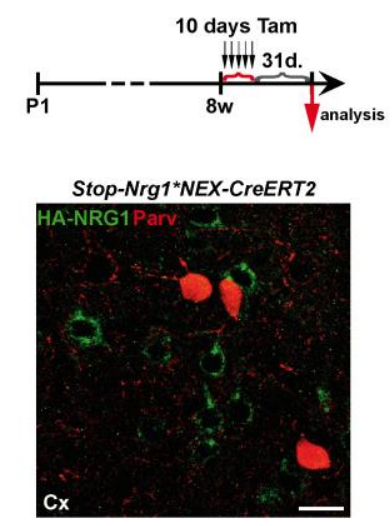

$\mathbf{E}$
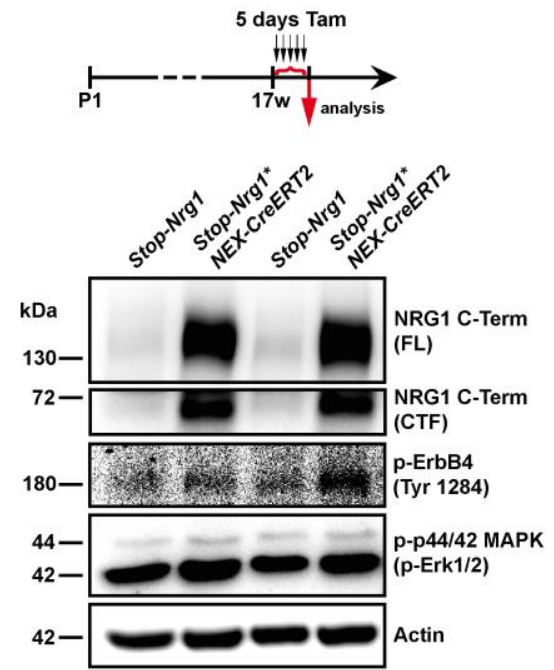

Fig. 29: Stop-Nrg1 ${ }^{\star} N E X-C r e E R T 2$ mice are a tool for tamoxifen-induced acute HA-NRG1 overexpression in adult mice and in vivo single-cell genetics. (A) Vibratome sections (bregma -1.7) of NEX-CreERT2*R26R-floxtdTomato mice that were injected with inclining tamoxifen concentrations (ranging from $25-100 \mathrm{mg} / \mathrm{kg}$ body weight) for 2 or 10 days at an age of 12 weeks and tissue was collected 50 days later. Tamoxifen injection at $100 \mathrm{mg} / \mathrm{kg}$ body weight for 10 days is considered a full induction. TdTomato reporter fluorescence confirmed increasing recombination in perfusion fixed, ranging from single recombined cells to full recombination of cortical projection neurons. Higher magnification confocal images of 2 days $50 \mathrm{mg} / \mathrm{kg}$ tamoxifen injected mice show single recombined pyramidal neurons of the cortex and hippocampus (locations marked by dashed boxes). Cx, cortex; Hipp, hippocampus. Scale bars, $30 \mu \mathrm{m}$. (B) Western blot analysis of hippocampus and thalamus protein lysates show HA-NRG1 overexpression in the hippocampus, but not in the thalamus of Stop-Nrg1*NEXCreERT2, resembling NEX expression pattern. 17 weeks old mice were injected with tamoxifen for 10 days at $100 \mathrm{mg} / \mathrm{kg}$ body weight and collected 31 days after the last injection. $\alpha$-Tubulin was used as a loading control. CTF, NRG1 C-terminal fragment; FL, full length; 
$\mathrm{kDa}$, kilodalton. (C) Fluorescent immunostaining for HA-NRG1 and PV on coronal paraffin section (bregma -1.7) of 3 months old Stop-Nrg1 ${ }^{*} N E X$-CreERT2 mice, reveals projection neuron-specific HA-NRG1 overexpression. Mice were injected with tamoxifen for 10 days at $100 \mathrm{mg} / \mathrm{kg}$ body weight and tissue was collected 1 month after the last injection. Cx, cortex. Scale bar, $20 \mu \mathrm{m}$. (D) For in vivo single cell genetics, 4 months old Stop-Nrg1*tdTomato*NEXCreERT2 triple transgenic mice were injected with tamoxifen for 2 days at $50 \mathrm{mg} / \mathrm{kg}$ body weight and tissue was collected 3 weeks after the last injection. While layer $\mathrm{V}$ pyramidal neurons of the cortex loose their GFP fluorescence due to recombination of the GFP-Stopflox cassette and gain tdTomato fluorescence (asterisk), hippocampal CA1 pyramidal neurons only partially loose their GFP fluorescence (\#). (E) Acute induction of HA-NRG1 overexpression in Stop-Nrg1 ${ }^{*} \mathrm{NEX}$-CreERT2 mice and western blot analysis of hippocampal protein lysates reveals HA-NRG1 overexpression, ErbB4 hyperphosphorylation and activation of MAPK signaling. 4 months old mice were injected with tamoxifen for 5 days at $100 \mathrm{mg} / \mathrm{kg}$ body weight and brain tissue was collected on the day after the last injection. $\beta$-actin was used as a loading control. CTF, NRG1 C-terminal fragment; FL, full length; kDa, kilodalton.

for HA-NRG1 and cell type specific markers confirmed that HA-NRG1 overexpression was restricted to cortical projection neurons (Fig. 29C).

In a pilot experiment to determine parameters for 'single cell genetics', StopNrg 1*NEX-CreERT2 mice were bred to R26R-floxtdTomato reporter mice. Triple transgenic mice and controls were injected with tamoxifen ( 2 days, $50 \mathrm{mg} / \mathrm{kg}$ ), based on the assumption that limited numbers of NEX-CreERT2-positive projection neurons recombine both the Stop-Nrg1 transgene and the R26R-floxtdTomato reporter, resulting in tdTomato fluorescent neurons that were no longer GFP-positive, but instead express HA-NRG1. Analysis of vibratome sections 3 weeks after tamoxifen injection revealed tdTomato-positive, GFP-negative cortical projection neurons (Fig. 29D). However, a subset of tdTomato-positive neurons of the hippocampal CA1 region maintained GFP fluorescence (Fig. 29D). This either suggests incomplete Cre-recombination of only one of the floxed cassettes or residual GFP fluorescence due to its long half-life.

To increase the 'acuteness' of the approach, western blot analysis was performed on protein lysates prepared one day after the last tamoxifen injection (5 days, 100 $\mathrm{mg} / \mathrm{kg}$ ). Western blotting with the Nrg1 C-terminal antibody revealed overexpression of full length and processed NRG1 already at this early timepoint (Fig. 29E). Testing for ErbB4 hyperstimulation with the anti-phospho-ErbB4 (Tyr1284) antibody identified a weak increase in ErbB4 receptor phosphorylation (Fig. 29E). Next, as a proof of concept it was addressed whether this approach allows to identify changes in downstream signaling cascades not present in chronic overexpression models (data not shown and Fig. 29B). Western blot analysis of hippocampal protein lysates showed a small increase in the phosphorylation of Erk1/2 in Stop-Nrg1 ${ }^{*} N E X$ CreERT2 mice, suggesting activation of the Raf-MEK-ERK signaling cascade (Fig. 29E). Thus, this 'acute' tamoxifen protocol could be suitable to investigate possible signaling cascades in the cortex downstream of NRG1/ErbB4 hyperstimulation. 


\subsection{Biochemical analysis of CRD-NRG1 signaling in NRG1 transgenic mice}

NRG1/ErbB4 signaling has been linked to SZ in numerous studies (Stefansson et al., 2002, 2003; Yang et al., 2003; Hahn et al., 2006; Law et al., 2007; Chong et al., 2008). The Icelandic at-risk haplotype (HaplCE) (Stefansson et al., 2002) has recently been associated with increased CRD-NRG1 expression in postmortem brain tissue of SZ patients (Weickert et al., 2012). CRD-NRG1 overexpression leads to chronic ErbB4 receptor hyperstimultation, ventricular enlargement, altered E/I balance, disrupted LTP, reduced PPI, and abnormal dendritic spine growth in transgenic mice (Agarwal et al., 2014). However, the molecular mechanisms of neuronal network dysfunctions downstream of NRG1/ErbB4 hyperstimulation have not been studied in detail.

Several mouse models of CRD-NRG1 overexpression based on Thy1.2 promoterdriven (Fig. 9, 10 and Agarwal et al., 2014) and Cre-dependent conditional activation (Fig. 11-28) are available. These mouse models show different phenotypes and allow the investigation of different aspects of NRG1 overexpression in vivo. To compare the underlying molecular changes side by side, a biochemical analysis was performed using conditional Stop-Nrg1 mouse models for embryonic (NEX-Cre-), postnatal (CamKII-Cre-) and acute (NEX-CreERT2-mediated) overexpression as well as "global" Thy1.2-driven full length HA-Nrg1-tg and BACE1 processed HA-Nrg $1^{\text {GIEF }}$ mice (Fig. 30B).

In previous experiments overexpression of CRD-NRG1 was shown to induce chronic ErbB4 hyperphosphorylation in several transgenic mouse models (Fig. 9B, 14B, 29E). To test the hypothesis that the amount of CRD-NRG1 overexpression determines the level of ErbB4 stimulation (Fig. 30A) hippocampal protein lysates from these mouse models were analyzed side by side by western blotting. This analysis revealed major differences in the level of HA-NRG1 overexpression, with the highest expression level observed in $\mathrm{HA}-\mathrm{Nrg} 1^{\mathrm{GIEF}}$ mice, followed by $\mathrm{HA}-\mathrm{Nrg} 1$-tg mice, whereas NEX-Cre- and CKII-Cre-based conditional mouse models showed moderate HA-NRG1 overexpression (Fig. 30C). The processed NTF in lysates derived from full length HA-NRG1 overexpressing mouse models matched the size of the HA-NRG1 variant expressed in $H A-N r g 1^{G I E F}$ mice, indicating in vivo processing in the stalk region by proteases, such as BACE1. Acute overexpression of HA-NRG1 in Stop$\mathrm{Nrg} 1{ }^{*} \mathrm{NEX}$-CreERT2 mice after 5 days of tamoxifen injection at $100 \mathrm{mg} / \mathrm{kg}$ and collection one day after the last injection resulted in weak overexpression compared 
A
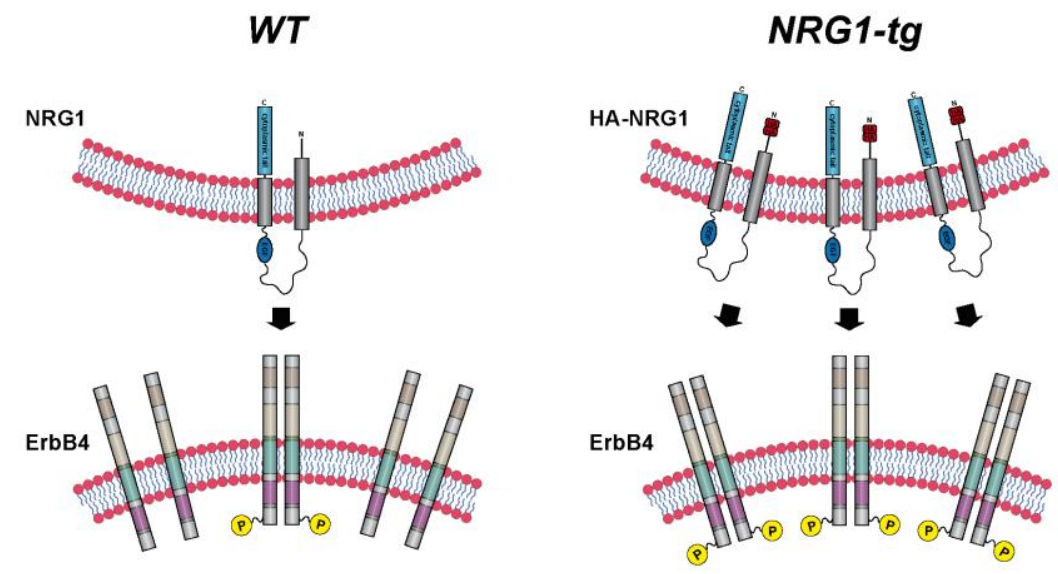

B

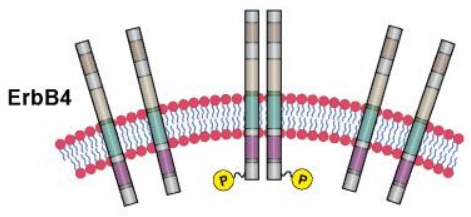

Conditional
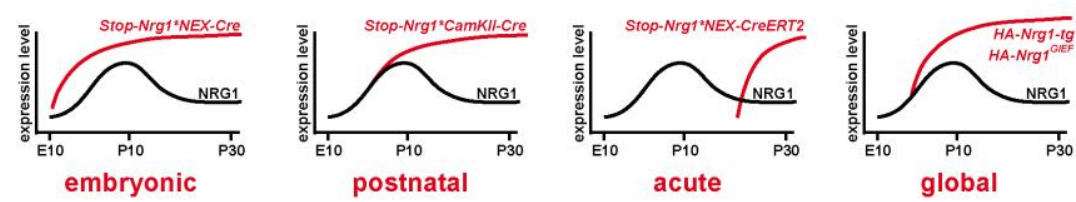

C

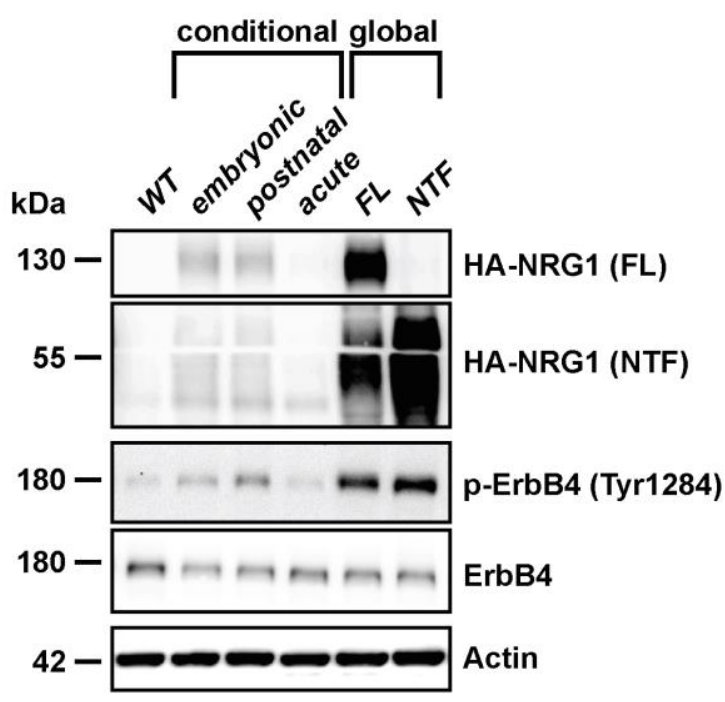

Fig. 30: NRG1 expression levels correlate with levels of ErbB4 hyperphosphorylation. (A) Our working hypothesis suggests that higher levels of NRG1 expression lead to higher ErbB4 receptor stimulation. P, phosphorylated tyrosine residues. (B) HA-NRG1 transgenic mouse models include conditional models that allow embryonic (NEX-Cre) and postnatal (CamKII-Cre) and tamoxifen-induced acute (Stop-Nrg ${ }^{*} \mathrm{NEX}$-CreERT2) activation of HA-Nrg1 overexpression. 'Global' models use the Thy1.2 promoter expression cassette and show high levels of HA-CRD-NRG1 or HA-NRG $1{ }^{\text {GIEF }}$ overexpression. Schematic expression curves illustrate onset and level of HA-NRG1 overexpression (red curves) in relation to endogenous levels (black curve). (C) Western blot analysis of conditional and global HA-NRG1 transgenic models side by side reveal different levels of HA-NRG1 overexpression and confirms correlating levels of ErbB4 receptor phosphorylation. Note that in all transgenic models ErbB4 total protein is reduced, indicating receptor internalization and degradation or gene regulation upon hyperstimulation. $\beta$-actin was used as a loading control. FL, full length; $\mathrm{kDa}$, kilodalton; $\mathrm{N}$-Term, Nrg1 N-terminal fragment. 
to the other models (Fig. 30C).

In summary, conditional mouse models express HA-NRG1 at more physiological levels when compared to Thy1.2-driven "global" transgenic mice (Fig. 30C). In line with the hypothesis that ErbB4 receptor phosphorylation correlates with HA-NRG1 expression (Fig. 30C), the highest ErbB4 phosphorylation level was observed in $H A$ $\mathrm{Nrg} 1^{\text {GIEF }}$ mice followed by HA-Nrg1-tg mice. As expected, conditional NRG1 transgenic models showed moderate ErbB4 phosphorylation levels, with the acute model displaying the lowest ErbB4 induction (Fig. 30C). Interestingly, concomitant with increased ErbB4 phosphorylation, a reduction in total ErbB4 protein (detected by a pan-ErbB4 antibody) was present in all transgenic mouse models, suggesting either internalization and degradation after receptor activation, or a downregulation of ErbB4 receptor expression upon hyperstimulation (Fig. 30C).

In summary, NRG1 transgenic mouse models at hand model distinct levels and profiles of HA-NRG1 expression and ErbB4 receptor activation.

\subsection{NRG1 forward and backsignaling - differential recruitment of LIMK1?}

NRG1-mediated canonical forward signaling proceeds via ErbB receptors. In addition to canonical forward signaling, backsignaling via the NRG1-ICD has been described in cultured cells (Bao et al., 2003; Mei and Xiong, 2008; Talmage, 2008; Chen et al., 2010; Fazzari et al., 2014; Mei and Nave, 2014). In addition to transcription factor functions after nuclear translocation (Bao et al., 2003), direct binding of the NRG-ICD to the serine/threonine kinase LIM kinase 1 (LIMK1) was observed (Wang et al., 1998). LIMK1 phosphorylates the actin depolymerizing factor cofilin and serves as a key regulator of actin cytoskeleton dynamics (Arber et al., 1998; Yang et al., 1998). LIMK1 has been implicated in synaptic maturation (Huang et al., 2000) and shuttles between the cytoplasm and the nucleus (Yang and Mizuno, 1999).

To further exploit transgenic mouse models, including backsignaling-deficient $H A$ $N r g 1^{\text {GIEF }}$ mice, for the investigation of forward and backsignaling-associated mechanisms, a comparative western blot analysis was performed using conditional Stop-Nrg1*NEX-Cre as well as "global" HA-Nrg1-tg and HA-Nrg1"GIEF mice. In agreement with previous findings, ErbB4 receptor phosphorylation correlated with the expression levels of different HA-NRG1 variants. As before, levels of total ErbB4 protein were reduced in all transgenic samples (Fig. 31A). Western blot analysis of hippocampal protein lysates used to examine effects in two major signaling pathways (Raf-MEK-ERK and PI3K-AKT-S6K pathways) downstream of NRG1/ErbB4 
forward signaling in cultured cells (Mei and Xiong, 2008; Mei and Nave, 2014), showed that in mice with chronic overexpression of full length HA-NRG1 (HA-Nrg1-tg and Stop-Nrg1*NEX-Cre mice) MAPK or PI3K signaling was not activated (Fig. 31B). However, both pathways were stimulated in $\mathrm{HA}-\mathrm{Nrg} 1^{\text {GIEF }}$ mice (Fig. 31B). This finding suggests more potent forward signaling functions of NRG1 NTF in HA-Nrg ${ }^{\text {GIEF }}$ mice, even under chronic conditions. In line with this finding, the NRG1 NTF was localized to axons of projection neurons with a higher abundance than the full length CRDNRG1 (Fig. 31B), similar to findings in the PNS (Velanac et al., 2011). Thus, the NRG1 NTF more likely reaches presynapses to signal to postsynaptic ErbB4 in interneurons.

Although the precise mechanisms are unknown, perturbed dendrite and axon growth of cortical neurons in CRD-NRG1 mouse mutants are thought to be mediated by NRG1-ICD backsignaling (Chen et al., 2008, 2010).

Immunostaining of paraffin sections from Stop-Nrg1*NEX-Cre mice with the anti-HA antibody revealed that HA-NRG1 accumulated in the somatodendritic compartment, whereas transport into the axonal and presynaptic compartment seemed to be limited (Fig. 31C). This lead to the working hypothesis that abnormal accumulation of full length HA-NRG1 in the somatodendritic compartment could lead to abnormal NRG1-ICD mediated backsignaling (Fig. 24C), with LIMK1 and cofilin as possible targets. Thus, hippocampal protein lysates from conditional Stop-Nrg1*NEX-Cre, as well as "global" $H A-N r g 1-\operatorname{tg}$ and $H A-N r g 1^{\text {GIEF }}$ mice were tested for LIMK1 and cofilin phosphorylation.

To verify the identity of the LIMK1 band, protein lysates from $L I M K 1^{-1 /}$ mice were included in the analysis. This analysis revealed that threonine residue 508 (important for the regulation of LIMK1 activity) was hypophosphorylated in Stop-Nrg ${ }^{*} N E X$-Cre mice, indicating reduced LIMK1 activity (Fig. 31C). In contrast, the phosphorylation

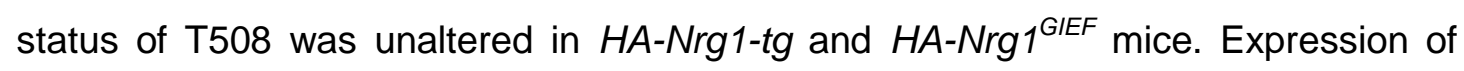
LIMK1 protein was unchanged in all transgenic NRG1 mouse models. As expected this band was absent in protein lysates from $L I M K 1^{-/}$mice (Fig. 31C). Examination of the phosphorylation status of cofilin at serine 3 revealed increased Ser3 phosphorylation in all NRG1 transgenic models analyzed (Fig. 31C). Conditional Stop-Nrg1*NEX-Cre mice showed stronger Ser3 phosphorylation than "global" $H A$ Nrg1-tg mice, which expressed the same HA-NRG1 variant even at higher levels. Unexpectedly, backsignaling-deficient $H A-N r g 1^{\text {GIEF }}$ mice showed the strongest Ser3 phosphorylation (Fig. 31C), implicating that NRG1 might regulate cofilin activity also via distinct forward directed signaling events. In none of the transgenic mouse models we did observe dysregulation of total cofilin protein (Fig. 31C). 
A
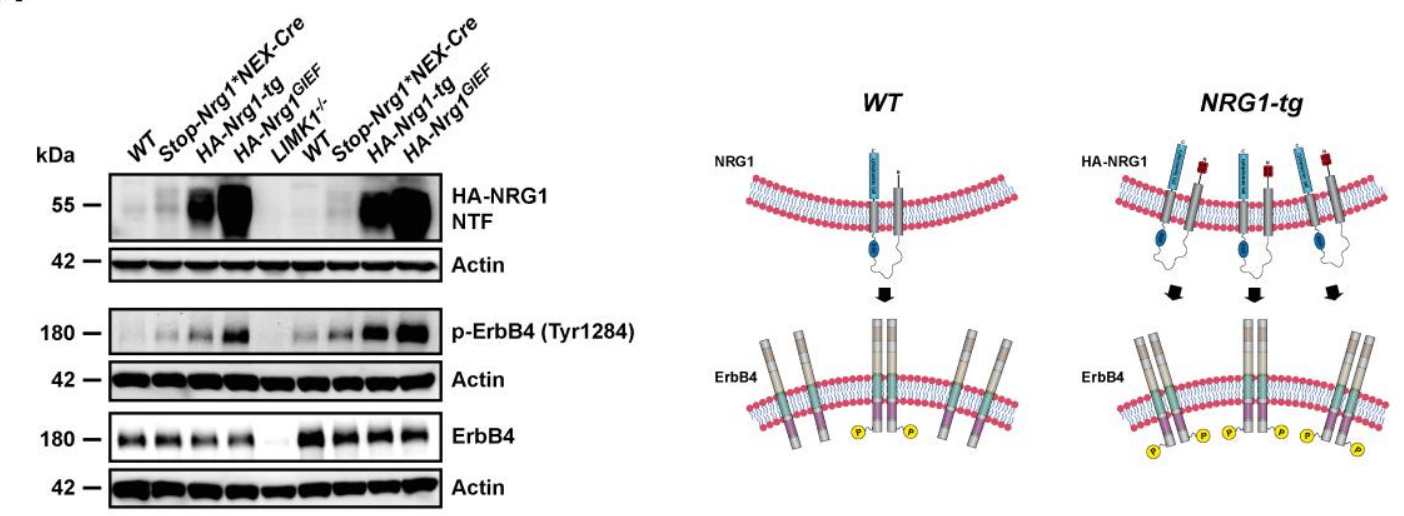

B
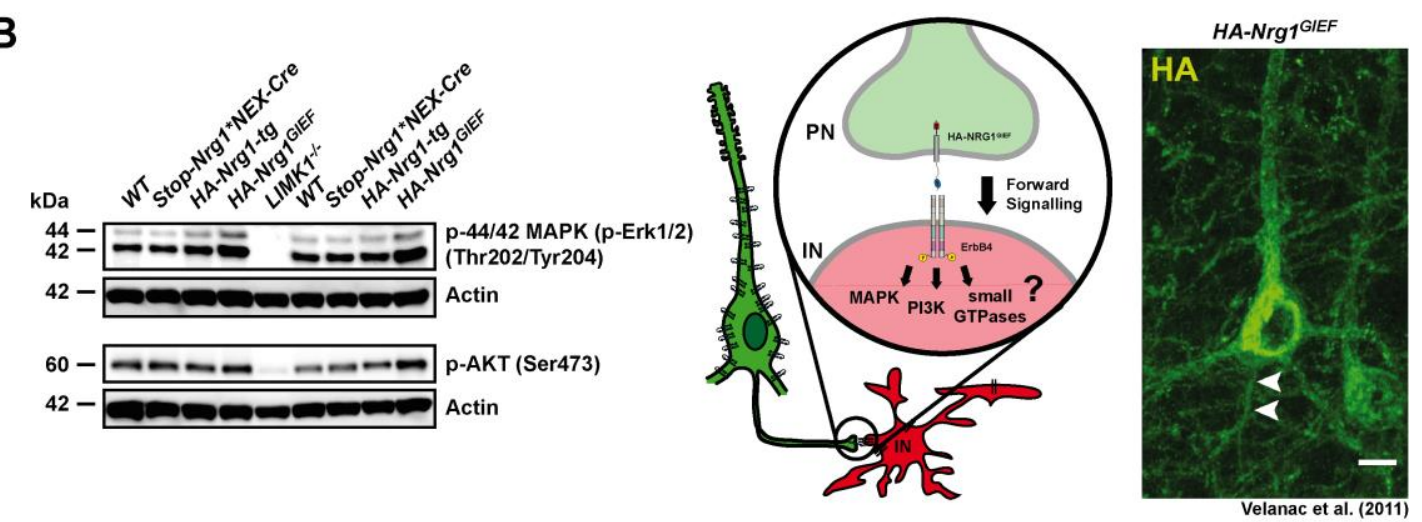

C
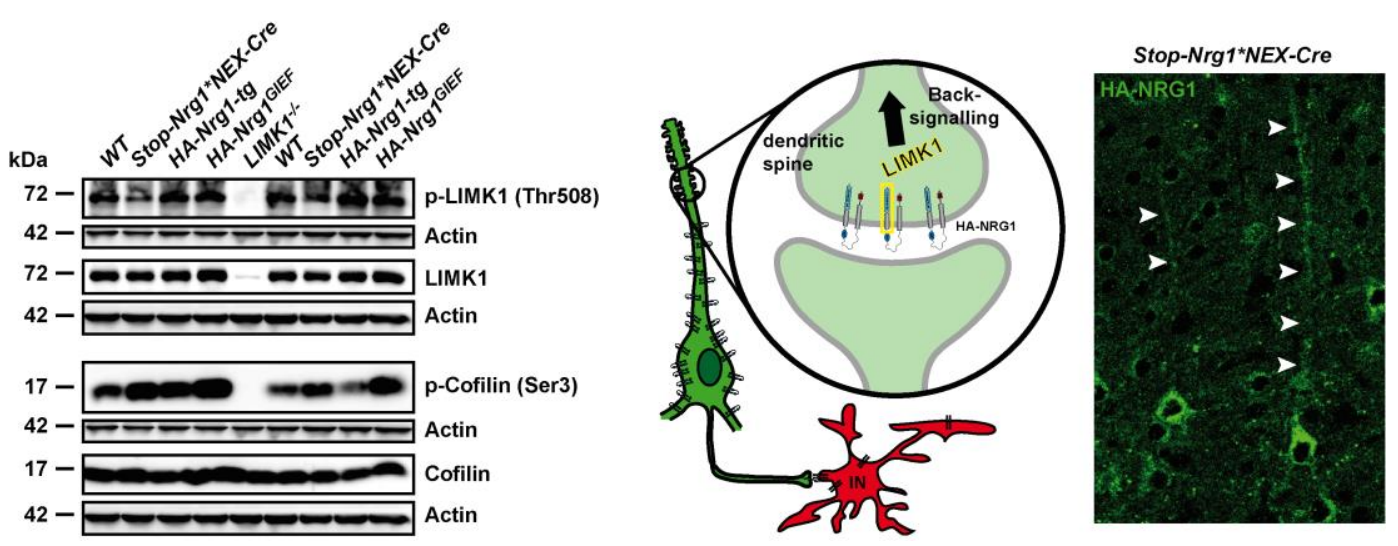

Fig. 31: A comparative analysis of NRG1 transgenic mouse models reveals differences in forward and backsignaling. (A) (left) Western blot analysis of hippocampal protein lysates of WT, conditional Stop-Nrg $1^{*} N E X$-Cre and global HA-Nrg1-tg and HA-Nrg $1^{\text {GIIEF }}$ mice reveals drastic differences in HA-CRD-NRG1 and HA-NRG1 $1^{\text {GIEF }}$ expression levels that correlates with ErbB4 receptor hyperphosphorylation levels, in line with our working hypothesis (right). ErbB4 total protein is reduced in all NRG1 transgenic mouse models. (B) (left) Western blot analysis of hippocampal protein lysates (as in A) shows increased MAPK and AKT phosphorylation in HA-Nrg $1^{\text {GIEF }}$ mice, but not in full length HA-CRD-NRG1 transgenic models, suggesting that the processed N-terminal fragment of CRD-NRG1 (containing the EGF-like domain) is the active part in canonical forward signaling. (Center) Illustration demonstration HA-NRG1 ${ }^{\text {GiEF }}$-mediated canonical forward signaling. (right) In line with this hypothesis, the HA-NRG1 $1^{\text {GIEF }}$ fragment was found in axonal structures in $H A$ $N r g 1^{\text {GIEF }}$ mice (Velanac et al., 2011). (C) (left) Western blot analysis of hippocampal protein lysates (as in A,B) reveals reduced LIMK1 phosphorylation in Stop-Nrg1*NEX-Cre mice, but not in 'global' models. $L I M K 1^{-\%}$ brain lysates were used to verify LIMK1 antibody reactivity. Cofilin phosphorylation is increased in all HA-NRG1 transgenic models. Total LIMK1 and cofilin protein levels are unaltered in HA-NRG1 transgenic models. (Center) LIMK1 is thought to mediate CRD-NRG1 backsignaling via the NRG1-ICD, potentially leading to altered 
dendritic spine dynamics by regulating the actin depolymerization activity of cofilin. However, backsignaling-deficient $H A-N r g 1^{G I E F}$ mice also show increased cofilin phosphorylation. (right) Fluorescent immunostaining for HA-NRG1 on a coronal paraffin section (bregma -1.7) of a Stop-Nrg1*NEX-Cre mouse shows accumulation of HA-CRD-NRG1 in the somatodendritic compartment and apical dendrites (arrow heads). $\beta$-actin was used as a loading control in all western blot experiments. FL, full length; kDa, kilodalton; IN, interneuron; N-Term, Nrg1 Nterminal fragment.

\subsection{HA-NRG1 is enriched in synaptosomes of Stop-Nrg1*NEX-Cre mice and regulates cofilin phosphorylation}

Chronically high levels of NRG1/ErbB4 hyperstimulation in Thy1.2-driven NRG1 transgenic mouse models could trigger unphysiological side effects and activate compensatory mechanisms that shut down signaling cascades. To specifically examine synaptic signaling functions of NRG1 that could underlay pathomechanisms of NRG1/ErbB4 hyperstimulation, crude synaptosomal fractions were prepared from Stop-Nrg $1{ }^{*} N E X$-Cre mice at 4 months of age and tested for changes in the LIMK1/cofilin pathway.

A synaptosomal purification method was adapted (Biesemann et al., 2014), which included homogenization of fresh tissue in $0.32 \mathrm{M}$ sucrose buffer, followed by differential centrifugation and sucrose gradient ultracentrifugation to extract a crude synaptosomal fraction with intact resealed synaptic terminals (Fig. 32A, see Methods for details). Purification steps for the preparation of crude synaptic membranes were skipped. Instead, gradient purified synaptosomes were used (Biesemann et al., 2014). An additional centrifugation step was applied to wash and pellet synaptosomes out of solution, to concentrate the solution, and redissolve in $0.32 \mathrm{M}$ homogenization buffer. To confirm the purification of synaptosomes, samples from purification steps were tested by western blotting for synaptic (synaptophysin, VGluT1) and glial markers (GLT-1, MBP) (Fig. 32B).

Synaptosomal preparations of Stop-Nrg1*NEX-Cremice and controls were tested for enrichment of HA-NRG1 by western blotting using an anti-HA antibody. As expected, HA-NRG1 was present in synaptosomes of Stop-Nrg1*NEX-Cre mice but not in controls. Both full length and processed HA-NRG1 was detected in synaptosomes (Fig. 32C). Preliminary analyses failed to detect phosphorylated ErbB4 receptors (Tyr1284) and LIMK1 (Thr508) in the synaptosomal fraction of transgenic mice or controls (data not shown). In contrast, phosphorylated cofilin (Ser3) was present in synaptosomes and clearly reduced in Stop-Nrg1*NEX-Cre mice, in contrast to total protein lysates from hippocampus (see Fig. 31C). This indicates differences in the subcellular targeting of phosphorylated cofilin to synapses under conditions of chronic NRG1 overexpressing (Fig. 32D). 
A

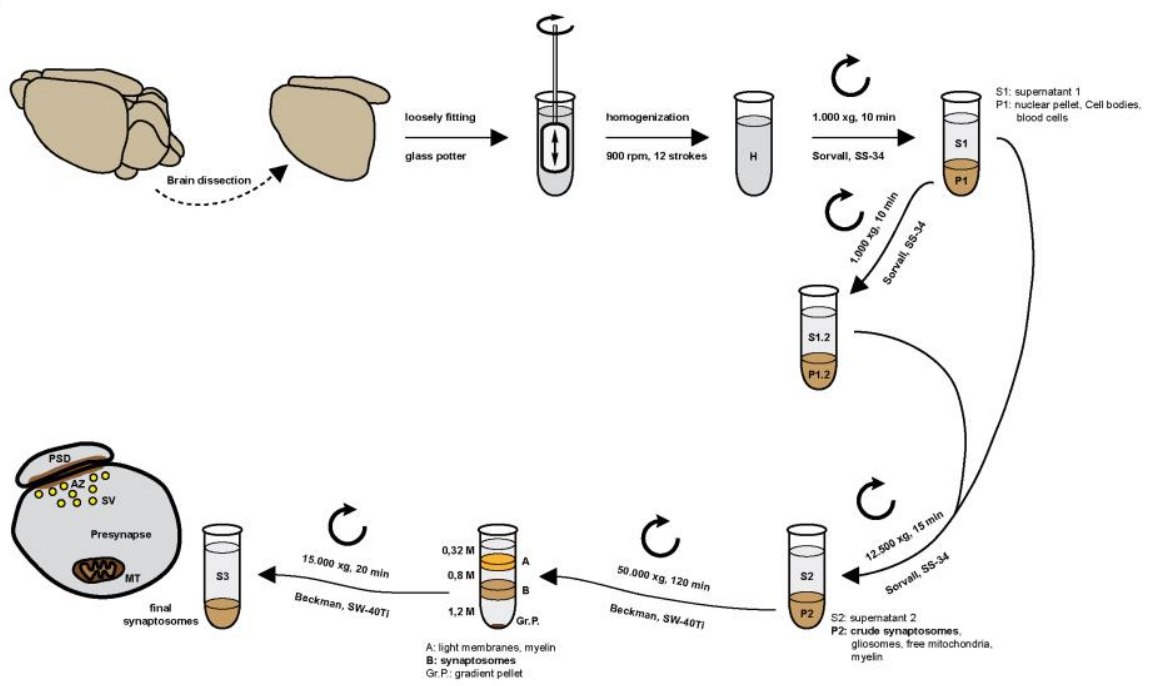

B

Stop-Nrg1*NEX-Cre

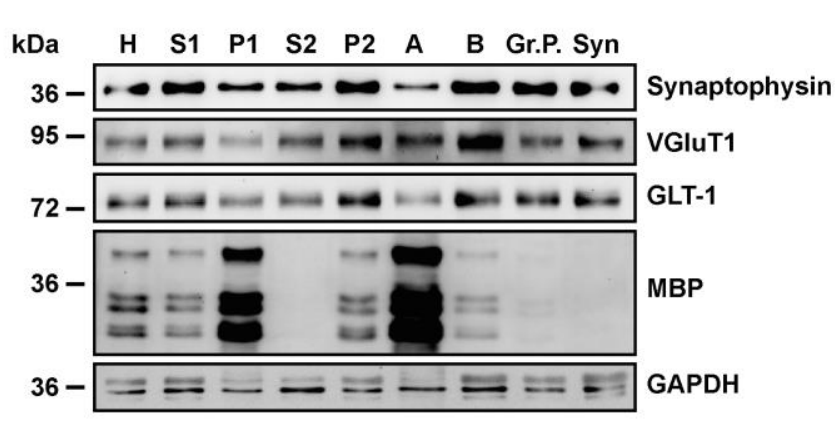

C

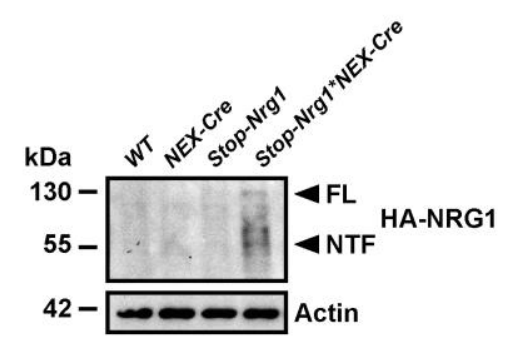

D

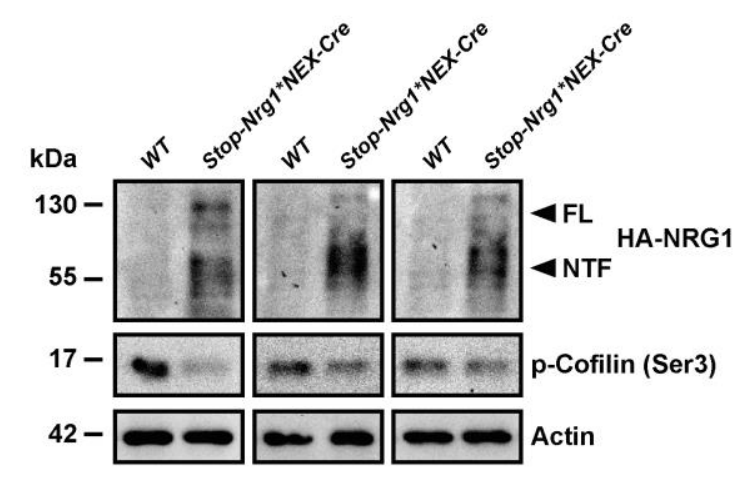

Fig. 32: HA-NRG1 is enriched in synaptosomes of Stop-Nrg1*NEX-Cre mice and regulates cofilin phosphorylation. (A) Schematic illustration of synaptosomal preparation as described in the methods section 6.4.3. (B) Western blot analysis of fractions of the synaptosomal preparation reveals concentration of synaptosomes, indicated by synaptophysin and VGluT1 immunoreactivity, and reduction of myelin (myelin basic protein, MBP) contamination in final synaptosomes (Syn). Glial glutamate transporter (GLT)-1 is also enriched in synaptosomal fractions, in line with the tripartite synapse theory. GAPDH was used as a loading control. (C) Western blot analysis of synaptosomal preparations reveals enrichment of full length and N-terminal fragment (NTF) of HA-CRD-NRG1 in synaptosomes of Stop-Nrg1*NEX-Cre mice. $\beta$-actin was used as a loading control. (D) Western blot analysis of synaptosomes reveals HA-NRG1 enrichment and reduction of cofilin phosphorylation in Stop-Nrg1 ${ }^{*} N E X$-Cre mice compared to $W T$ controls. $\beta$-actin was used as a loading control. $\mathrm{FL}$, full length; kDa, kilodalton; NTF, Nrg1 N-terminal fragment. 


\section{Discussion}

Human NRG1 and ErbB4 genes are possible genetic risk factors for schizophrenia (SZ) (Stefansson et al., 2002; Nicodemus et al., 2006; Silberberg et al., 2006; Law et al., 2007). NRG1/ErbB4 signaling regulates multiple aspects of nervous system development, including myelination, interneuronal migration and synaptic plasticity (Mei and Xiong, 2008). Most NRG1 at-risk haplotypes are located in the non-coding region of the gene (Stefansson et al., 2002; Weickert et al., 2012), suggesting that rather than disturbed protein function, altered NRG1 expression could underlay a pathomechanism with relevance for SZ. Indeed both increased and decreased NRG1 expression has been observed in postmortem brain of SZ patients (Law et al., 2006; Bertram et al., 2007). The icelandic haplotype (HapICE) has been associated with increased expression of NRG1 type III (CRD-NRG1), the most abundant NRG1 isoform in the human brain (Liu et al., 2011), and was correlated with an earlier onset of the disease (Weickert et al., 2012). However, the contribution of NRG1/ErbB4 signaling to disease pathology and the precise pathomechanisms underlying SZ remain elusive. Together, these findings suggest a working hypothesis according to which NRG1/ErbB4 hyperstimulation causes neuronal network dysfunctions with relevance for SZ.

The aim of this study was to address this working hypothesis by analyzing different loss- and gain-of-function mouse models of NRG1, with a special focus on interneuron development and behavioral functions. In addition, a novel conditional transgenic mouse line was employed, which allowed Cre-dependent stage- and cell type-specific activation of HA epitope-tagged CRD-NRG1. This mouse line was used to investigate the role of NRG1 in early embryonic versus postnatal development, and to model acute changes of NRG1/ErbB4 signaling in the adult brain. Histological, biochemical and behavioral experiments were performed to identify and compare the functional involvement of stage-specific NRG1 overexpression in the generation of schizophrenia-relevant phenotypes. Data derived from this Ph.D. project have recently been published in the journal Cell Reports (Agarwal et al., 2014).

\subsection{Embryonic NRG1 signaling is dispensable for interneuron migration}

Reduced numbers of GABAergic interneurons have been reported in the post mortem brain of SZ patients (Benes et al., 1991; Chance et al., 2005; Holt et al., 2005; Levitt, 2005; Lewis et al., 2005; Heckers and Konradi, 2014), and the ErbB4 null mutation in mice leads to impaired interneuron migration and a subsequent 
reduction of GABAergic interneurons in the cortex (Neddens and Buonanno, 2009). This suggests that NRG1 (via signaling to ErbB4) serves a role in interneuron development and migration, and that diminished NRG1 signaling could contribute to inhibitory network dysfunctions in SZ. This hypothesis was tested by breeding conditional NRG1 knockout mice (Li et al., 2002) to Emx1-Cre driver mice (Gorski et al., 2002), which eliminate NRG1 from glutamatergic neurons, astrocyes and oligodendrocytes, but not interneurons, beginning at E10. Unexpectedly, these NRG1 mutants survived into adulthood and showed no difference in the number or cortical position of GAD67 ${ }^{+}$interneurons. Moreover, inhibitory neurotransmission in the adult hippocampus was increased, not diminished, in the absence of NRG1 (Agarwal et al., 2014), in contrast to findings in ErbB4 mutants (Fazzari et al., 2010). Thus, NRG1 expression in the embryonic cortex is dispensable for the development and migration of GABAergic interneurons, but is required for the fine-tuning of excitatory and inhibitory (E/I) neurotransmission. The discrepancy to findings in ErbB4 mutants suggests compensatory functions by other ErbB4 ligands, such as NRG2 (Carraway et al., 1997) or NRG3 (Zhang et al., 1997) during the regulation of inhibitory circuits. This idea is supported by the absence of obvious brain abnormalities in NRG2 null mutants (Britto et al., 2004) and could be genetically addressed in compound mutants of NRG family members.

\subsection{CRD-NRG1 overexpression hyperstimulates ErbB4 receptors and alters inhibitory neurotransmission}

Elevated expression of CRD-NRG1 has been reported in a postmortem cohort of SZ patients carrying the HapICE haplotype (Weickert et al., 2012), associated with SZ (Stefansson et al. in 2002). To model chronically elevated CRD-NRG1 expression, Thy1.2 promoter-driven NRG1 transgenic mice (Nrg1-tg; Michailov et al., 2004) were examined, which express CRD-NRG1 in neurons of the neocortex and hippocampus, but also in subcortical regions and the PNS (Agarwal et al., 2014). ErbB4 hyperphosphorylation has been observed in postmortem brain of SZ patients (Hahn et al., 2006). In line with this, we found sustained ErbB4 hyperphosphorylation at Tyr1284 in hippocampal protein lysates of CRD-NRG1 transgenic mice, making CRD-NRG1 transgenic mice a suitable tool to study the consequences of NRG1/ErbB4 hyperstimulation in vivo.

CRD-NRG1 has been reported to serve as a permissive guidance signal for the migration of interneurons from the subpallium to the cortex (Flames et al., 2004). 
However, total numbers of $\mathrm{GAD}^{+} 7^{+}$and $\mathrm{PV}^{+}$interneurons were unaltered in the cortex and hippocampus of Nrg1-tg mice at P14, but when numbers of GAD67 ${ }^{+}$ interneurons were assessed based on their position within cortical layers, a significant reduction in layer IV of Nrg1-tg mice was observed. Cortical layer IV (or internal granular layer of the cortex) receives its main input from thalamocortical and intra-hemispheric corticocortical afferents (Jones, 1998). Tangential migration of cortical interneurons and the outgrowth of thalamocortical axons functionally interact in the ventral telencephalon and are co-regulated by NRG1 signaling (López-Bendito et al., 2006). Thus, abnormal NRG1 signaling in the subcortical regions could alter thalamocortical axon pathfinding, thereby preferentially affecting interneuron migration into layer IV. Distinct from findings in postnatal brains, in vivo imaging of $\mathrm{PV}^{+}$interneurons in adult $\mathrm{Nrg} 1-\operatorname{tg}^{*} \mathrm{PV}$-GFP mice by 2P-LSM revealed a reduction of $\mathrm{PV}\left(\mathrm{GFP}^{+}\right)$interneurons in layer II/III and $\mathrm{V}$ of the cortex. A similar reduction of $\mathrm{PV}$ $\left(\mathrm{GFP}^{+}\right)$interneurons was observed in a second mouse line (HA-Nrg1-tg* $\left.P V-G F P\right)$, strongly supporting the absence of transgene integration-associated effects. Taken together, overexpression of CRD-NRG1 impairs the normal intergration and/or maintenance of $\mathrm{PV}^{+}$interneurons in the neocortex. However, in the absence of obvious neuroinflammation and -pathology (Brinkmann et al., 2008; Velanac et al., 2011; Agarwal et al., 2014), it is currently unclear if the reduction of PV (GFP ${ }^{+}$) interneurons is due to reduced cell numbers, e.g. following apoptotic cell death, or changes in the expression of the PV promoter, as GFP expression was used for quantification. To address these questions and to establish a possible link between postnatal and adult findings, further studies, including immunostaining and 2P-LSM at different time points are required.

Altered neurotransmission could contribute to dysregulation of calcium-binding proteins, such as PV, or a reduction in the expression of GAD67 (Akbarian et al., 1995; Hashimoto et al., 2003; Ongür et al., 2010). This idea is supported by electrophysiological recordings of pyramidal neurons in Nrg1-tg mice, which showed disrupted plasticity (STP and LTP) at the Schaffer collateral-CA1 synapse and a shift of the excitatory/inhibitory $(E / I)$ balance towards enhanced inhibition. This effect could be due to increased number of synaptic inputs from GABAergic interneurons or increased GABA release from inhibitory presynapses (Agarwal et al., 2014). These data suggest stage-specific deficits in GABAergic interneuron intergration and maintenance, and an imbalance of the $\mathrm{E} / \mathrm{l}$ ratio. Impaired interneuron functions in cortical layers II/III and $\mathrm{V}$ could influence cortical connectivity and output to subcortical areas, such as basal ganglia, and thereby influence behavior of mice. 


\subsection{CRD-NRG1 transgenic mice show SZ-relevant behavioral dysfunctions}

CRD-NRG1 overexpression in the PNS severely compromises the neuromuscular junction in Nrg1-tg mice (W.J. Thompson, personal communication), which prevents behavioral analysis. Therefore, we examined instead a mouse line (HA-Nrg1-tg) in which HA epitope-tagged CRD-NRG1 is expressed under control of the same Thy1.2 cassette as in Nrg1-tg mice. HA-Nrg1-tg mice displayed pronounced anxiety-like behavior in the open field test, indicating that NRG1 overexpression might change circuits involved in emotional states. In line with this assumption, increased CRDNRG1 expression was observed in the amygdala of HA-Nrg1-tg mice. Moreover, broad expression of ErbB4 receptors was reported in PV-negative cells of the amygdala, and ErbB4 $4^{-/}$mice, but not conditional $P V-E r b B 4^{-/}$mice exhibit reduced anxiety (Shamir et al., 2012). The anxiogenic effect seems to be isoform-specific, as Ig-NRG1 transgenic mice show no increase in anxiety-like behavior in the open field test (Deakin et al., 2009). Prepulse inhibition (PPI) is an operational measure of sensorimotor gating and PPI deficits are frequently observed in neuropsychiatric disorders (Kohl et al., 2013), possibly reflecting abnormalities in frontal-corticalstriatal circuitry (Swerdlow and Geyer, 1998; Young et al., 2010). When analyzed in the PPI test, HA-Nrg1-tg mice exhibited an increased startle response and reduced PPI. Reduced PPI was also observed in $\lg -\mathrm{Nrg} 1$ transgenic mice (Deakin et al., 2009; Yin et al., 2013), indicating that Ig-NRG1 and CRD-NRG1 may serve similar roles in the regulation of PPI circuitry. Deficits in PPI (which were absent in conditional NRG1 mutants) are a hallmark for schizophrenia, further supporting the hypothesis that NRG1/ErbB4 hyperstimulation contributes to SZ pathology.

Taken together, histological, physiological, and behavioral studies in NRG1 mutants and transgenic mice suggest that an "optimal" level of NRG1 signaling is required for efficient synaptic neurotransmission and possibly higher brain functions, thereby extending an ,inverted U-shaped' model (Role and Talmage, 2007) to a ,bell-shaped' model (Agarwal et al., 2014). In addition, these data imply that human NRG1 at-risk polymorphisms exert a gain-of-function effect.

\subsection{Conditional overexpression of CRD-NRG1 in transgenic mice}

The NRG1 gene encodes at least 30 different isoforms. NRG1 isoforms are differentially expressed during development, indicating distinct functions in the 
developing and adult nervous system (Liu et al., 2011). Results obtained in Thy1.2 promoter-driven NRG1 transgenic mice suggest that hyperstimulated NRG1/ErbB4 signaling, rather than NRG1 hypofunction, could contribute to SZ pathology. SZ is believed to be a neurodevelopmental disorder (Harrison, 1999), but it is not clear how altered NRG1/ErbB4 signaling could increase susceptibility for SZ. To address the complexity of endogenous NRG1 signaling, a novel transgenic mouse line was generated, which allows conditional CRD-NRG1 overexpression based on the CreloxP system. The transgene cassette (Stop-Nrg1) contained a fragment of the chicken $\beta$-actin promoter, driving expression of CRD-NRG1 after Cre-mediated removal of a ,floxed' Stop-cassette encoding GFP. Virtually all tissues expressed GFP, including brain, spinal cord, muscles and heart. Thus, Stop-Nrg1 mice could serve as a genetic tool to study NRG1 functions also outside the nervous system, e.g. during heart development, or in Hirschsprung disease (HSCR), a congenital disorder of the gastrointestinal tract, characterized by the absence of intramural ganglion cells, for which increased NRG1 expression has been reported (GarciaBarcelo et al., 2009; Tang et al., 2011, 2012a, 2012b; Luzón-Toro et al., 2012; Phusantisampan et al., 2012; Gui et al., 2013).

Fluorescent immunostainings for cell type-specific markers revealed that not all neural cell types in the brain expressed the Stop-Nrg1 transgene (based on GFP expression). The GFP cassette was predominantly expressed in pyramidal neurons and oligodendrocytes, but only by few interneurons, micro- and astroglia, similar to findings by others (M. Rossner, personal communication). Thus, the chicken $\beta$-actin promoter fragment is not well suited for expression in interneurons, which prevented studies on NRG1 autocrine signaling in ErbB4 ${ }^{+}$interneurons. The main glial cell type expressing the transgene were oligodendrocytes $\left(\sim 80-90 \% \mathrm{GFP}^{+}\right)$, whereas only $\sim 40-50 \%$ of microglia and astrocytes were $\mathrm{GFP}^{+}$. Taken together, the chicken $\beta$ actin promoter fragment used for the generation of Stop-Nrg1 mice appears not to be suitable for studies in interneurons, astrocytes and microglia. A possible alternative approach would be to target the Rosa26 locus using homologous recombination, as in the case of the R26R-floxtdTomato reporter mouse line (Madisen et al., 2010).

\subsection{Modeling stage-specific CRD-NRG1 overexpression in cortical projection neurons}

Endogenous CRD-NRG1 is predominantly expressed by glutamatergic projection neurons in the brain, starting during embryonic stages (Liu et al., 2011). To model 
cortical projection neuron-restricted overexpression of HA-NRG1 beginning at embryonic stages (E12), Stop-Nrg1 mice were bred to NEX-Cre driver mice (Goebbels et al., 2006). Fluorescent immunostaining confirmed projection neuronrestricted HA-NRG1 expression, starting at early embryonic stages. Immunostaining for the N- (HA-tag) and C-terminus of HA-NRG1 showed overlapping signals, which accumulated inside and on the surface of the somatodendritic compartment and apical dendrites of projection neurons, indicating either trafficking of full length HANRG1 or coordinated transport of the cleaved N- and C-terminus fragments to these subcellular domains. To which extend HA-NRG1 (or cleaved fragments) are transported to presynaptic sites is under current investigation, but preliminary results support limited axonal and presynaptic transport, similar to findings in the PNS (Velanac et al., 2011). Biochemical analysis by western blotting of hippocampal protein lysates from Stop-Nrg1*NEX-Cre mice confirmed expression of full length and proteolytically processed HA-NRG1, as two protein bands ( 140 kDa, $60 \mathrm{kDa})$ were detected, albeit at lower levels compared to Thy1.2 promoter-driven mouse lines. In line with our working hypothesis, conditional overexpression of HA-NRG1 lead to increased steady state levels of ErbB4 phosphorylation.

In a complementary approach CamKII-Cre driver mice (Minichiello et al., 1999) were used to direct HA-NRG1 overexpression to cortical projection neurons starting at postnatal stages (P5). This approach aimed at modeling a postnatal onset of hyperstimulated NRG1/ErbB4 signaling, according to the late neurodevelopmental onset hypothesis of SZ (Harrison, 1999). Similar to the NEX-Cre model, HA-NRG1 expression was restricted to projection neurons of the cortex and hippocampus. However, Stop-Nrg $1^{*} \mathrm{CKII}$-Cre mice displayed additional recombination in subcortical regions, e.g. striatum, thalamus, and hypothalamus. Western blot analysis confirmed expression of full length and processed HA-NRG1 and ErbB4 hyperphosphorylation. Taken together, Stop-Nrg1 transgenic mice in combination with different Cre-driver lines allow stage- and cell type-specific CRD-NRG1 overexpression and ErbB4 hyperstimulation.

\subsection{Embryonic and postnatal HA-NRG1 overexpression is not associated with neuropathology or -inflammation at adult stages}

NRG1 is a growth and differentiation factor involved in many neurodevelopmental processes, including neuronal migration and myelination, but has also been implicated in cancer (Talmage, 2008; Buac et al., 2009; McIntyre et al., 2010). 
Transgenic overexpression of a growth factors could have pathological and neurotoxic effects. Stop-Nrg1 mice also permanently express GFP in most cells of their body, and GFP expression was shown to trigger pronounced hypomyelination and axonal pathology in transgenic mice, which express GFP under control of the CNP promoter (Millet et al., 2012). To identify possible signs of neuroinflammation or -degeneration, an immunohistochemical analysis was performed, which included a quantification of interneuron numbers in embryonic (Stop-Nrg1*NEX-Cre) and postnatal (Stop-Nrg $1^{*} \mathrm{CKII}-\mathrm{Cre}$ ) models at four months and one year of age. Postnatal onset of HA-NRG1 overexpression in Stop-Nrg1 ${ }^{*} \mathrm{CKII-Cre} \mathrm{mice} \mathrm{had} \mathrm{no}$ effect on $\mathrm{GAD} 67^{+}$and $\mathrm{PV}^{+}$interneuron numbers.

When cell numbers were quantified in Stop-Nrg1*NEX-Cre mice, a tendency for reduced cell numbers was counted in Stop-Nrg1 ${ }^{\star} N E X$-Cre but also in NEX-Cre mice. In addition, a modestly increased cortical width was observed in both StopNrg ${ }^{*} N E X$-Cre and NEX-Cre mice. Together, increased cortical width and slightly reduced cell numbers resulted in significantly reduced cell densities in Stop$\mathrm{Nrg} 1^{*} \mathrm{NEX}$-Cre and NEX-Cre mice. Thus, effects on interneuron numbers were not derived from CRD-NRG1 overexpression.

Furthermore, a mildly increased area of $\mathrm{IBA} 1^{+}$microglia was noticed in the hippocampus of NEX-Cre mice at 1 year of age, suggesting microglial activation. This effect was significant when compared to the other genotypes, except for Stop$N r g{ }^{*} N E X$-Cre mice, which themselves were not significantly different from other controls, indicating that HA-NRG1 overexpression was even beneficial and may counteract microglia activation. Since microglia activation was not detected in CKIICre mice, changes in IBA $1^{+}$microglia were not simply the result of Cre expression. It is currently unclear if the loss of one functional gene copy in NEX-Cre mice, which causes NEX haploinsufficiency, could effect cortical size, e.g. by reducing tissue stability during the processing of brain sections. Impaired neuronal development was not reported for the NEX null mutation (Schwab et al., 1998; Goebbels et al., 2006), however aged heterozygous and homozygous NEX-Cre mutants will be studied in more detail, as this is a widely used Cre driver line.

These histological studies demonstrate absence of neurotoxic, inflammatory or mitogenic effects resulting from conditional CRD-NRG1 overexpression or permanent expression of GFP in conditional transgenic mice. This is consistent with findings in Thy1.2 promoter-based transgenic mice with much higher NRG1 expression, which also lack signs of neuroinflammation or -degeneration. Different from Thy1.2 promoter-based transgenic mice, these studies have not shown 
changes in the number or location of cortical interneurons in Stop-Nrg1 ${ }^{*} N E X$-Cre or Stop-Nrg $1{ }^{*} \mathrm{CKII}-\mathrm{Cre}$ mice, consistent with the finding that subcortical NRG1 expression domains play an important role in mediating interneuron migration (Flames et al., 2004).

In stark contrast to cortical neurons, overexpression of different NRG1 variants, including CRD-NRG1, in Schwann cells of the PNS causes severe Schwann cell hyperplasia (Huijbregts et al., 2003; R. Stassart, personal communication), possibly as a result of autocrine signaling to glial ErbB2/ErbB3 receptors. A comparable experiment of CRD-NRG1 overexpression in interneurons (with prominent ErbB4) is currently not possible due to limited expression of the conditional transgene in interneurons.

\subsection{Thy1.2-driven CRD-NRG1 mice have enlarged ventricles and reduced body weight}

Enlarged ventricles are the most replicated endophenotype in SZ (Harrison, 1999) and variants of the NRG1 gene have been associated with increased lateral ventricle volume (Mata et al., 2009). Alterations in ventricular sizes were observed in loss- and gain-of-function mouse models by MRI imaging, with Emx-Cre ${ }^{*} \mathrm{Nrg} 1^{t / f}$ mice having reduced, and Nrg1-tg mice enlarged lateral ventricles (Agarwal et al., 2014). Interestingly, ventricular enlargment seems to result from isoform-specific functions, as Ig-Nrg1 transgenic mice have normal ventricular sizes (P. Harrison, personal communication). A comparative histological examination of ventricular sizes in all available CRD-NRG1 transgenic mouse lines revealed enlarged lateral ventricles in HA-Nrg1-tg mice, similar to Nrg1-tg mice (Agarwal et al., 2014). Interestingly, HA$N r g 1^{\text {GIEF }}$ mice and conditional transgenic mice (Stop-Nrg1*NEX-Cre and Stop$\mathrm{Nrg} 1^{\star} \mathrm{CKII}$-Cre mice) had normal lateral ventricles. This suggests that the backsignaling-deficient $H A-N r g 1^{G I E F}$ is not capable to trigger ventricular enlargement, even though it is expressed at even higher levels than full length HA-NRG1 in HANrg1-tg mice. The finding that cortical-restricted HA-NRG1 overexpression had no effect on ventricular sizes, indicates that subcortical CRD-NRG1 overexpression is required to induce ventricular enlargement. The general overexpression level in conditional mice is lower than in Thy1.2-driven mice, however Stop-Nrg ${ }^{*} \mathrm{CKII-Cre}$ mice, which show expression in striatal and thalamic regions, are not significantly different from HA-Nrg1-tg mice. Overexpression of CRD-NRG1 in cells of the choroid plexus could also directly affect liquor production. HA-NRG1 is not expressed in the 
choroid plexus in conditional Stop-Nrg1 ${ }^{\star} \mathrm{NEX}$-Cre and Stop-Nrg $1{ }^{*} \mathrm{CKII}-\mathrm{Cre}$ mice, however HA-Nrg1-tg mice show positive signals in chromogenic immunostaining for the HA tag in the choroid plexus, and expression of the Thy1.2 promoter in the choroid plexus has been reported (Campsall et al., 2002). In addition, ErbB4 expression has also been shown for large cells in the choroid plexus (Bean et al., 2014). If CRD-NRG1 overexpression in the choroid plexus plays a role in regulating ventricular sizes requires further investigations. Taken together, subcortical or choroid plexus overexpression of NRG1 (via currently undefined signaling pathways) could lead to ventricular enlargement in Nrg1-tg and HA-Nrg1-tg mice.

Since Nrg1-tg mice appeared to have reduced body weight, a comparative analysis of body weight was performed for all CRD-NRG1 mouse lines. Reduced body weight was observed in Nrg1-tg, HA-Nrg1-tg and Stop-Nrg $1^{*} \mathrm{CKII-Cre} \mathrm{mice.} \mathrm{However,} \mathrm{Stop-}$ $\mathrm{Nrg} 1{ }^{\star} \mathrm{CKII}$-Cre mice were only significantly different from Stop-Nrg ${ }^{\star} \mathrm{NEX}$-Cre mice, which showed normal body weight compared to controls. Again, subcortical overexpression of CRD-NRG1 seems to affect body weight. The mechanisms underlying these weight reductions could involve NRG1-mediated effects on leptin levels, as injection of the recombinant EGF-like domain of NRG1 was shown to affect leptin levels, food intake and body weight in normal-weight mice (Ennequin et al., 2015). ErbB4 expression was found in the hypothalamus and the Raphe Nuclei (Bean et al., 2014), and reduced preweaning bodyweight has been reported in conditional Nestin-Cre ${ }^{\star} E r b B 4^{\text {null }}$ mice (Golub et al., 2004). The Raphe Nuclei regulate food intake, memory retention and anxiety (Carlini et al., 2004). Thus, Raphe Nuclei could be an important target for the regulation of body weight or the generation of anxiety-like behavior observed in HA-Nrg1-tg mice. The hypothalamus is also known to control metabolism, thermoregulation, and stress response. Expression of ErbB4 in $\mathrm{GAD}^{+} 7^{+}$interneurons has been reported in the hypothalamus (Bean et al., 2014), and could serve as a link between altered body weight and stress levels in HA-Nrg1$\operatorname{tg}$ mice. However, whether hyperstimulated NRG1/ErbB4 signaling ultimately alters food intake in transgenic mice needs further evaluation.

\subsection{Embryonic HA-NRG1 overexpression induces hyperactivity, but no anxiety-like behavior or PPI deficits}

'Global' CRD-NRG1 overexpression in HA-Nrg1-tg mice lead to pronounced anxietylike behavior and impaired PPI (Agarwal et al., 2014), as well as working memory deficits in the $\mathrm{Y}$-maze test (Wehr et al., in preparation). To examine specific effects of 
the temporal and spatial aspects of NRG1 overexpression on behavior, Stop$\mathrm{Nrg} 1{ }^{*} \mathrm{NEX}$-Cre and Stop-Nrg $1{ }^{*} \mathrm{CKII}-\mathrm{Cre}$ mice were analyzed in the same set of behavior tests.

Unexpectedly, no signs of anxiety-like behavior were found in both conditional models. Instead, both lines showed hyperactivity, which is thought to correspond to psychomotor agitation in schizophrenic patients and is considered a schizophreniarelevant behavior (Snyder, 1973; Yin et al., 2013a). Hyperactivity was found in the open field and the hole board test and was more pronounced in Stop-Nrg1 *NEX-Cre mice. In the hole board test hyperactivity even seemed to prevent mice from exploring the new environment, as they showed tendencies to reduced exploration time and hole visits. Also in the tail suspension test Stop-Nrg1 ${ }^{*} N E X$-Cre mice fought more against the aversive situation, in line with hyperactivity. Stop-Nrg $1{ }^{*} N E X$-Cre mice showed the strongest hyperactivity always at the beginning of a test, arguing for novelty-induced hyperactivity. In line with these findings, hypoactivity was observed in conditional CKII-Cre*Nrg1/f/f (Agarwal et al., 2014) and Nestin-Cre ErbB4 ${ }^{\text {null }}$ mutants (Golub et al., 2004). Hyperactivity was also described in conditional transgenic mice with modest Ig-NRG1 overexpression in the cortex (Yin et al., 2013a), interestingly, these mice also show no anxiety-like behavior. However, transgenic mice with Thy1.2-driven strong cortical and subcortical Ig-NRG1 overexpression show normal motor behavior with initial hypoactivity (Deakin et al., 2009). These contrasting findings in transgenic mice expressing the same NRG1 isoform. Indicate that differences in expression levels (and thereby ErbB4 stimulation) or cortical versus subcortical functions play an important role in NRG1mediated regulation of motor behavior. NRG1 expression level differences might differentially regulate serotonine and/or dopamine signaling in the striatum and basal ganglia, including the Nucleus accumbens, thought to be involved in regulation hyperlocomotion (Taepavarapruk et al., 2000; Bishop and Walker, 2003; Brus et al., 2004; Fadda et al., 2005). Interestingly, these circuits might also be involved in the regulation of anxiety levels in these mice (Scott et al., 2006; Jiang et al., 2015).

No PPI impairments were observed in Stop-Nrg1 ${ }^{*} \mathrm{NEX}$-Cre and Stop-Nrg1 ${ }^{\star} \mathrm{CKII}-\mathrm{Cre}$ mice, in contrast to HA-Nrg1-tg mice (Agarwal et al., 2014). In addition to unaltered PPI, both lines exhibited normal startle responses. Absence of PPI impairments could be related to lower cortical overexpression of HA-NRG1 in both lines compared to HA-Nrg1-tg mice, which is associated with a lower level of ErbB4 hyperphosphorylation. This idea is supported by the finding that administration of spironolactone ameliorates the PPI deficits in HA-Nrg1-tg mice. Spironolactone is a novel modulator of ErbB4 activity, which restores normal levels of ErbB4 
phosphorylation in the brain of HA-Nrg1-tg mice (Wehr et al., in preparation). In addition, absence of HA-NRG1 overexpression from subcortical regions in conditional mouse lines, notably the striatal, could explain normal PPI as striatal projections have been considered important for the generation of PPI (Baldan Ramsey et al., 2011). PPI deficits appear not to be NRG1 isoform-specific as both Thy1.2 promoter-driven (Deakin et al., 2009) and conditional transgenic mice with lgNrg1 overexpression exhibit impaired PPI (Yin et al., 2013). Finally, in line with a bellshaped model of NRG1-mediated regulation of sensorimotor gating, PPI deficits also occur in heterozygous CRD-NRG1 mutants (Chen et al., 2008). Stop-Nrg1 *NEX-Cre and Stop-Nrg $1{ }^{*} \mathrm{CKII}$-Cre mice also showed no deficits in working memory, as measured in the Y-maze test. This is in contrasts to reduced performance of $H A$ Nrg1-tg mice in this test. Similar to PPI, administation of spironolactone to HA-Nrg1$\operatorname{tg}$ mice ameliorates this deficit (Wehr et al., in preparation), suggesting that the level of NRG1/ErbB4 hyperstimulation is critical for the magnitude of working memory deficits.

Finally, deficits were observed in cued fear memory in Stop-Nrg1 ${ }^{*} N E X$-Cre mice. A tendency to reduced contextual fear memory was also observed. Impaired contextual and cued fear conditioning was also found in CKII-Cre ${ }^{*} \mathrm{Nrg}^{t / f}$ mice (Agarwal et al., 2014), again supporting a bell-shaped model of NRG1 functions in hippocampal learning. However, considering the hyperactivity observed in Stop-Nrg1*NEX-Cre mice, deficits in fear conditioning could be independent from hippocampal learning deficits, but simply linked to hyperactivity-mediated reduction in freezing behavior.

In contrast to HA-Nrg1-tg mice (Wehr et al., in preparation), Stop-Nrg1 ${ }^{*} N E X-C r e$ and Stop-Nrg $1{ }^{*} \mathrm{CKII}-\mathrm{Cre}$ mice showed normal pain sensitivity in the hotplate test, arguing for effects of Thy1.2 promoter-driven expression of HA-Nrg1 in the PNS.

Similar to Stop-Nrg $1{ }^{*} \mathrm{NEX}$-Cre mice, Stop-Nrg $1{ }^{*} \mathrm{CKII-Cre} \mathrm{mice} \mathrm{displayed} \mathrm{a} \mathrm{tendency}$ for hyperactivity in the open field and hole board test, but performance in all other tests was unaltered. Thus, postnatal overexpression of HA-NRG1 exerts only minor effects on mouse behavior. However, as the Stop-Nrg $1{ }^{*} \mathrm{CKII-Cre} \mathrm{sample} \mathrm{was} \mathrm{small}$ ( $\mathrm{n}=7$ Stop-Nrg ${ }^{*} \mathrm{CKII}-\mathrm{Cre}$ mice), this experiment can only be considered as a pilot study, which requires replication in a larger cohort. 


\subsection{Stop-Nrg ${ }^{\star}$ NEX-CreERT2 mice allow acute NRG1 overexpression in the adult brain}

Acute stimulation of NRG1/ErbB4 signaling may provide a better insight into 'physiological' functions of NRG1 in the brain. To model acute stimulation of NRG1/ErbB4 signaling in the brain, NEX-CreERT2 driver mice were used, which allow tamoxifen-induced activation of Cre recombinase in cortical projection neurons (Agarwal et al., 2011).

Tamoxifen injection (for 10 days at $100 \mathrm{mg} / \mathrm{kg}$ bodyweight, defined as the maximum recombination treatment) of Stop-Nrg ${ }^{*} N E X$-CreERT2 mice and harvest of protein samples one month later resulted in approximately half of the HA-NRG1 expression observed in Stop-Nrg1*NEX-Cre mice. Reduced HA-NRG1 expression in Stop$\mathrm{Nrg}{ }^{*} N E X$-CreERT2 mice could be due to incomplete recombination in projection neurons and absent Cre activity in adult dentate gyrus granule cells (Agarwal et al., 2011). In contrast to HA-Nrg1-tg and Stop-Nrg1*NEX-Cre mice, when Stop$N r g 1{ }^{*} N E X-C r e E R T 2$ mice were tamoxifen injected for maximum recombination for 5 days, and tissue was collected the day after the last injection, MAPK signaling (based on increased Erk1/2 phosphorylation) was induced. This is the first example for NRG1-mediated activation of a signaling pathway in a transgenic mouse model. Under conditions of chronic overexpression in HA-Nrg1-tg and Stop-Nrg1 ${ }^{\star} N E X$-Cre mice, feedback mechanisms seem to effectively downregulated activation of this signaling pathway. Subsequently, increased Erk1/2 phosphorylation was also found in $H A-N r g 1^{\text {GIEF }}$ mice, in line with the hypothesis that BACE1 processing activates NRG1, such that NRG1-mediated Erk1/2 activation overrides negative feedback mechanisms, even under chronic conditions. In summary, Stop-Nrg1*NEX-CreERT2 mice open up a way to analyze acute NRG1/ErbB4 signaling in the adult brain and therefore could be used as a model to test late onset hyperstimulation of this signaling module in the development of SZ. A comparable approach of acute activation of Ig-NRG1 signaling has recently shown the development and regression of SZ-relevant phenotypes upon activation or inactivation of lg-Nrg1 overexpression (Yin et al., 2013a).

Using sensitive R26R-floxtdTomato Cre reporter mice, parameters were established for the production of small numbers of recombined cortical neurons based on limited tamoxifen administration. This should have provided the basis for single-cell genetics. Unexpectedly, administration of low doses of tamoxifen to Stop-Nrg1 ${ }^{*} N E X$ CreERT2*tdTomato mice to examine individual tdTomato ${ }^{+} / \mathrm{HA}-\mathrm{NRG} 1^{+}$projection neurons resulted in activation of either one or the other transgene, but not both at the 
same time. Similar observations were made in PLP-CreERT2 mice (A. Agarwal, personal communication), the reason for this, however, remains unclear. In addition, many tdTomato ${ }^{+}$neurons in the hippocampus maintain GFP fluorescence, indicating insufficient concomitant recombination of the Stop-Nrg1 transgene.

\subsection{Possible roles of NRG1-mediated forward and backsignaling in cortical network dysfunction}

Several CRD-NRG1 transgenic mouse lines are available, which express different levels of CRD-NRG1 in the brain. Compared to Thy1.2-driven transgenic models, conditional Stop-Nrg1 mice show more physiological expression levels. Importantly, sustained ErbB4 hyperphosphorylation at Tyr1284 was detected in the cortex of all analyzed NRG1 transgenic mouse lines, and correlated with the level of NRG1 overexpression over a wide range of expression levels. This shows that ErbB4 is not rate limiting for NRG1 signaling in the brain, consistent with findings in the PNS, where heterozygous NRG1 mutants, but not heterozygous ErbB2/ErbB3 mutants are hypomyelinated (Michailov et al., 2004). The finding of permanently increased steady-state levels of hyperphosphorylated ErbB4 in hippocampal protein lysates under conditions of chronic NRG1 overexpression also implies that mechanisms for the downregulation of ErbB receptor activity, e.g. dephosphorylation by phosphatases, including receptor phosphotyrosine phosphatase $\beta$ (RPTP $\beta$ ), or PTPN21, both of which have been associated with SZ (Buxbaum et al., 2008; PlaniLam et al., 2015), or receptor inactivation by proteolysis or internalization, are not sufficient to compensate for NRG1-mediated activation. In line with this, it was shown that ligand-mediated endocytosis of ErbB4 receptors via the clathrin pathway is slow compared to the EGF receptor, and that hippocampal interneurons predominantly express the TACE-mediated cleavage-resistant JMb ErbB4 receptor isoforms, and thus, maintain a high level of ErbB4 at the cell surface (Longart et al., 2007). However, we found reduced levels of total ErbB4 protein in hippocampus protein lysates of all analyzed NRG1 transgenic mouse lines. In line with this, it was shown that internalization of stimulated ErbB4 receptors is important and necessary for downstream signaling (Liu et al., 2007), and ErbB4 receptor levels can be regulated by ubiquitination by neuregulin receptor degradation protein-1 (Nrdp1), that was shown to suppress ErbB3 and ErbB4 receptor levels when coexpressed in COS7 cells (Diamonti et al., 2002).

Robust ErbB4 hyperphosphorylation was identified at Tyr1284, which is implicated as 
a SHC1 adapter protein binding site, involved in MAPK signaling (Schulze et al., 2005). Whether other tyrosine residues are also hyperphosphorylated, e.g. Tyr984 (STAT5-binding) or Tyr1056 (PI3K-p85 binding) (Schulze et al., 2005; Kaushansky et al., 2008), remains to be analyzed in future studies and could reveal activation of additional signaling pathways.

Previous histological studies in HA-Nrg1-tg and HA-Nrg1 ${ }^{\text {GIEF }}$ mice revealed differential subcellular localization of full length and BACE1-processed CRD-NRG1 (HA-NRG1 ${ }^{\text {GIEF}}$ ). While full length CRD-NRG1 was observed mainly in the somatodendritic compartment, $\mathrm{HA}-\mathrm{Nrg} 1^{\text {GIEF }}$ was also found in distal dendritic processes and in axons (see also Velanac et al., 2011). Moreover, whereas full length CRD-NRG1 can mediate forward and backsignaling, $\mathrm{HA}-\mathrm{NRG} 1^{\text {GIEF }}$ lacks backsignaling activity. In combination with different subcellular localization, full length CRD-NRG1 and HANRG $1^{\text {GIEF }}$ might exhibit different signaling properties. Based on these findings a comparative biochemical analysis of several NRG1 transgenic lines was performed to examine whether distinct CRD-NRG1 variants, associated with different levels of NRG1/ErbB4 hyperstimulation, recruit different signaling pathways.

Western blot analysis of HA-Nrg1-tg, Stop-Nrg1*NEX-Cre and HA-Nrg ${ }^{\text {GIEF }}$ mice revealed that only in $H A-N r g 1^{\text {GIEF }}$ mice MAPK and PI3K signaling pathways were hyperactivated, supporting the hypothesis that BACE1 processing activates NRG1 and is a limiting factor for NRG1 signaling in the brain. As aforementioned, in acute Stop-NRG1*NEX-CreERT2 mice that were collected directly the day after the last tamoxifen injection, activated MAPK signaling was also found, indicating that under acute stimulation of NRG1 signaling the activation of forward signaling by proteolytic cleavage might be different than under chronic conditions, and in addition, downregulation of signaling pathways via feedback mechanisms might be involved under chronic full length CRD-NRG1 overexpression conditions. To further address this hypothesis and to rule out signaling effects simply due to higher HA-NRG GIEF expression, homozygous HA-Nrg1-tg mice with very high HA-CRD-NRG1 expression could be produced and tested for activated MAPK and PI3K signaling. It is however interesting that $\mathrm{HA}-\mathrm{NRG} \mathrm{GIEF}^{\mathrm{G}}$ induces these pathways even under chronic overexpression, which could also be due to the differential trafficking of the two variants. To which extend differential activation of these signaling pathways has further morphological or functional (physiological and behavioral) consequences in $H A-N R G^{G I E F}$ mice requires further investigations. Accordingly, examination of dendritic spines by STED microscopy and electrophysiological analysis of synaptic plasticity in the hippocampus of $H A-N r g 1^{\text {GIEF }}$ mice have been initiated. In summary, $H A-N R G^{G I E F}$ mice provide the opportunity to analyze specific functions of NRG1- 
mediated forward signaling.

In addition to canonical forward signaling, backsignaling mediated by the ICD of NRG1 was already described more than 10 years ago (Bao et al., 2003), however not much is known about its role in the regulation of cortical development. Similar to ErbB4, $\gamma$-secretase-mediated cleavage in the intramembrane region of NRG1 releases the NRG1-ICD, which can translocate into the nucleus, where it has been implicated in the regulation of neuronal survival (Bao et al., 2003), oligodendroglial differentiation (Pirotte et al., 2010), and PSD95 expression in the mouse cochlea (Bao et al., 2004). In line with this, it was shown that a substitution at the intramembranous valine 321 to leucine, that was previously linked to SZ (Walss-Bass et al., 2006), disrupted intramembranous proteolytic cleavage by $\gamma$-secretase and abolished ICD signaling of CRD-NRG1, which resulted in defects of growth and branching of dendrites. This function was shown to be even independent of ErbB kinase activity (Chen et al., 2010). In addition, loss of the $\gamma$-secretase subunit Aph1B was shown to impair synaptic neurotransmission and plasticity, and dendritic spine development, and this effect was rescued by co-expression of NRG1-ICD in a cellautonomous fashion (Fazzari et al., 2014). In addition to the proposed transcription factor-like functions of the NRG1-ICD, a direct interaction of the NRG1-ICD with LIMK1 was reported in a yeast two-hybrid screen (Wang et al., 1998). LIMK1 is a major regulator of cytoskeleton remodeling (Arber et al., 1998; Yang et al., 1998) and spine dynamics (Meng et al., 2002, 2003; Sarmiere and Bamburg, 2002). Overexpression of $\lg -\mathrm{Nrg} 1$ in transgenic mice was shown to recruit LIMK1 into synaptosomes, to enhance cofilin phosphorylation, and to impair glutamatergic neurotransmission (Yin et al., 2013a). We found dysregulated expression of cytoskeleton remodeling proteins and abnormal spine growth in Thy1.2 promoterdriven CRD-NRG1 mice (Agarwal et al., 2014). Together, this suggested a possible interaction of CRD-NRG1 backsignaling with LIMK1. Biochemical analysis showed a reduction in LIMK1 phosphorylation in hippocampal lysates of Stop-Nrg1 ${ }^{*} N E X$-Cre mice, but not in HA-Nrg1-tg and HA-Nrg ${ }^{\text {GIEF }}$ mice. Levels of total LIMK1 protein were similar in all groups. In addition, increased levels of cofilin phosphorylation were observed in all of these transgenic mice. HA-Nrg1 $1^{\text {GIEF }}$ mice showed the strongest induction of cofilin phosphorylation, whereas HA-Nrg1-tg mice displayed only a modest increase. These results are currently difficult to interpret as the textbook knowledge claims that phosphorylation activates LIMK1, which leads to increased cofilin phosphorylation, thereby inhibiting cofilin and stabilizing filamentous actin (Scott and Olson, 2007; Mizuno, 2012; Sparrow et al., 2012). Nevertheless, reduced 
LIMK1 phosphorylation in Stop-Nrg1*NEX-Cre mice is consistent with the idea that selective accumulation of full length CRD-NRG1 in the (postsynaptic) somatodendritic compartment may trigger abnormal backsignaling to LIMK1, thereby affecting its phosphorylation status. Importantly, western blot analysis of synaptosomal fractions showed a decrease of phosphorylated cofilin in StopNrg1*NEX-Cre mice compared to controls. This finding is in contrast to our observations in total hippocampal lysates, but indicates subcellular compartmentalization with regard to NRG1 regulated downstream signaling. Thus, upregulation of CRD-NRG1 under disease conditions may alter subcellular trafficking (and processing) of the full length protein, resulting in accumulation in the somatodendritic compartment and abnormal backsignaling. This could include local reduction in LIMK1 and cofilin phosphorylation. In line with this, it has been shown that other actin-binding proteins, including debrin, profiling II and $\mathrm{N}$-catenin are recruited into dendritic spines upon synaptic activity. Accumulation of profilin II in dendritic spines for instance, was shown to persist for hours beyond initial stimulation, depends on postsynaptic $\mathrm{Ca}^{2+}$ levels, and stabilizes spine morphology in a mature state (Ackermann and Matus, 2003). To further address the potential subcellular differences in LIMK1 and cofilin localization, primary neuronal cell cultures of NRG1 transgenic mice will be used in future studies.

Increased cofilin phosphorylation in backsignaling-deficient $\mathrm{HA}-\mathrm{Nrg}^{\text {GIEF }}$ mice in the absence of altered LIMK1 phosphorylation strongly suggests that forward signaling in $\mathrm{HA}-\mathrm{Nrg} 1^{\text {GIEF }}$ mice triggers mechanisms that ultimately regulate cofilin phosphorylation via distinct mechanisms, for instance inhibition of slingshot phosphatases (Niwa et al., 2002; Spratley et al., 2011; Mizuno, 2012). In addition, the Ras family GTPases and their downstream MAP kinase signaling pathways are known to also regulate dendritic spine morphology (Wu et al., 2001; Gärtner et al., 2005), thus, in HA-Nrg1 ${ }^{\text {GIEF }}$ mice with enhanced MAPK signaling cofilin phosphorylation could be regulated via MAPK signaling (with low expression of ErbB4 in projection neurons). Differential stimulation of cofilin phosphorylation in Stop-Nrg ${ }^{*} N E X$-Cre and HA-Nrg1-tg mice indicates that different levels of CRDNRG1 overexpression might trigger distinct signaling pathways or differentially activate distinct feedback-loops. In the future, it will be important to further analyze if direct interactions of the NRG1-ICD and LIMK1 indeed take place, thereby bypassing upstream members of the signaling cascade, e.g. p21-activated kinase 1 (PAK1) or Myosin light chain 2 (MLC2) (Scott and Olson, 2007; Barnes and Polleux, 2009). A direct interaction of the NRG1-ICD with LIMK1, depended or independent of ErbB4, in the regulation of dendritic spines and synaptic plasticity could be an important 
pathomechanism underlying SZ pathogenesis and would underline the importance of multimodal NRG1 signaling properties.

Taken together, the current working model suggests that NRG1 forward signaling is executed by the $\mathrm{N}$-terminal product (NRG $1^{\mathrm{GIEF}}$ ) of BACE1 cleavage, which activates ErbB4 receptors mainly in GABAergic interneurons to modulate inhibitory neurotransmission. Backsignaling via the NRG1-ICD, partly independent from ErbB4, regulates gene expression and morphological changes in pyramidal cells, including dendritic spines. This could influence synaptic plasticity and integrity, and potentially involve interactions with LIMK1, but also other interaction partners, for instance microtubule-associated serine/threonine kinases (MAST) and syntrophin-associated serine/threonine kinase (SAST) kinases (Cary Lai, unpublished data). Other data suggest an ErbB4-independent mechanism regulating spine dynamics via kalirin-7, a key regulator of spine remodeling (Cahill et al., 2013). In line with this, loss of kalirin7 was also shown to influence locomotor activity and PPI in kalirin-7 mutant mice (Cahill et al., 2009). Kalirin-7 is a brain-specific GEF for Rho-like small GTPases, and by directly activating Rac1, and subsequent regulation of PAK1 activity it can be linked to LIMK1/cofilin signaling (Scott and Olson, 2007; Penzes and Jones, 2008). However, by direct interaction with LIMK1, NRG1 could directly impact on the actin cytoskeleton and spine dynamics.

In summary, considering the complexity of NRG1 signaling further studies of NRG1 isoforms under chronic and acute situations, as well as in different cortical areas and cell types, will be of great importance for the understanding of pathomechanisms of SZ. In this sense, Thy1.2-driven HA-Nrg1-tg mice serve as an „easy access“ model for hyperstimulated NRG1/ErbB4 signaling, which is suitable for drug screening (Wehr et al., in preparation). Stop-Nrg1 mice on the other hand, allow the investigation of specific pathomechanisms associated with Cre-mediated stage- and cell type-specific HA-NRG1 overexpression. Thus, global' and conditional NRG1 transgenic mice are valuable research tools for a better understanding of the pathomechanisms involved in neuropsychiatric disorders, such as schizophrenia. 


\section{Materials}

\subsection{Kits and chemicals}

All chemicals were purchased from Sigma-Aldrich and Merck unless stated otherwise. All molecular biology and DNA purification kits were purchased from Qiagen, Invitek, BIORAD, Applied Biosystems, Promega, Stratagene, Sigma-Aldrich, Macherey-Nagel and nexttec ${ }^{\mathrm{TM}}$. General laboratory materials were purchased from Gilson, Sarstedt, Molecular Bio Products, Greiner Bio One, Falcon and Eppendorf.

\subsection{Websites referred for online protocols}

Neuroscience http://mrw.interscience.wiley.com/emrw/9780471142300/home/

Molecular biology http://mrw.interscience.wiley.com/emrw/9780471142720/home/

Protein http://mrw.interscience.wiley.com/emrw/9780471140863/home/

\subsection{Equipment}

\section{Lab water systems}

Sartorius Arium 611

\section{Pipettes}

Hirschmann Laborgeräte pipetus-akku

Gilson Pipetman $(2 \mu \mathrm{l}, 10 \mu \mathrm{l}, 20 \mu \mathrm{l}, 100 \mu \mathrm{l}, 200 \mu \mathrm{l}, 1000 \mu \mathrm{l})$

\section{Deep-freeze storage}

New Brunswick Scientific Co. UltraLow Temperature Freezer U725

\section{DNA preparation and analysis}

Advanced Biotechnologies Ltd. CombiThermosealer

Biometra Thermocycler T3

Biometra TGradient 96-well

Eppendorf Centrifuge 5415 R

Eppendorf Thermomixer T3

Heraeus Biofuge Pico

INTAS UV-system with Camera and printer

Memmert waterbath

ÖTTI waterbath

New Brunswick Scientific Innova 4000 Inkubator Shaker

Heidolph Titramax 1000 T-shaker with Incubator 1000

Sartorius Extend finebalance 
Scientific Industries Inc. G-560E Vortex Genie-2

Sharp R-202 microwave

Qiagen Sigma 4K15C centrifuge

\section{Proteinbiochemistry}

Beckman Ultra Centrifuge

Bertin Technologies Precellys 24 Lysis \& Homogenization with Peqlab Tubes

BIORAD PowerPac 300 Powersupply

BIORAD Mini Protean 3 electrophoresis chamber

BIORAD Mini Trans-Blot ${ }^{\circledR}$ cell

Beckman Centrifuge Tubes

Hecht Assistant 348 RM5 Rotating mixer

IKA KS 260 basic shaker

Intas ChemoCam Imager ECL HR-16-3200

New Brunswick Scientific Co. TC-7 Tissue culture roller

Pharmacia LKB-EPS 500/400 Powersupply

Sartorius S 5ml Glas-Teflon Potter and B.Braun Potters Homogenisator

Sorvall Centrifuge

Sorvall Rotor SS-34

SW-41Ti swing out bucket rotor

ThermoMax Molecular Devises Microplate Reader

\section{Perfusion of mice}

Heidolph Pump Drive PD 5101 Peristaltic Pump

In-house made preparation platform with drainage

ÖTTI waterbath

Pharmacia Fine Chemicals Peristaltic Pump P-1

\section{Histology}

Daewoo microwave

Inolab wtw Series pH720 pH-meter

Leica Jung Cryocut CM3000

Leica Mikrotom VT1000S vibratome

Microm AP280-1/-2/-3 paraffin embedding centre

Microm HM400 sliding microtome

Microm HMP110 embedding station

PFM waterbath 1000

Polyscience Inc. tissue cassettes IV 


\section{Microscope and binoculars}

Leica Confocal AOBS SP2 inverted CLSM

Leica MZ16F fluorescent stereomicroscope

Zeiss Imager.Z1 with Power Supply 231, XBO 75W HBO 100W lamp, AxioCam MRc camera and Zeiss Zen 2012 (blue edition) software

Zeiss Observer.Z1 AX10, HXP120 UV-lamp, Power Supply 23, SMC 2009 stage control, ApoTome.2, AxioCam MRm camera and Zeiss Zen 2012 (blue edition) software

2P-LSM custom-made, equipped with an fs-pulsed titanium-sapphire laser (Chameleon Ultra II, Coherent, Glasgow, UK) and a long-distance 20x/1.0 NA water immersion objective (Zeiss, Jena, Germany)

\section{Mouse behaviour}

Digital video camcorder (Sony)

Fear conditioning setup (TSE Systems, Bad Homburg, Germany)

Light dark preference test chamber - inhouse build

Open field test Plexiglas arena with hole board inserts and ActiMot Software (TSE Systems, Bad Homburg, Germany)

San Diego Instruments' SR-Lab startle response system

Ugo Basile Series 46000 Fear Conditioning System

Y-maze continuous alteration test - inhouse build

Panasonic Lumix DMC-TZ31 digital camera

\section{Computers and Softwares}

Apple MacBook 13“, 2.4 GHz Intel Core 2 Duo with Mac OS X Version 10.6.8

Adobe Illustrator CS3, Version 13.0.2

Adobe Photoshop CS3, Version 10.0.1

(FIJI Is Just) ImageJ, Version 1.0

GraphPad Prism 5 for Mac OS X, Version 5.0b

Mekentosj Papers, Version 1.9.3

Microsoft Excel 2008 (Version 12.3.6) and 2011 (Version 14.0.0) for Mac

Microsoft Word 2011 for Mac, Version 14.0.0 


\subsection{Molecular biology buffers and stock solutions}

50x Tris-Acetat EDTA (TAE) buffer

$$
2.0 \mathrm{M} \quad \text { Tris-Acetate, } \mathrm{pH} 8.0
$$$$
50 \mathrm{mM} \quad \text { EDTA }
$$

$57.1 \mathrm{ml} \quad$ Glacial acetic acid

Adjust volume to $1000 \mathrm{ml}$ with $\mathrm{H}_{2} \mathrm{O}$.

1x Tris-EDTA (TE) buffer

$$
\begin{array}{ll}
10 \mathrm{mM} & \text { Tris- } \mathrm{HCl}, \mathrm{pH} 8.0 \\
1 \mathrm{mM} & \text { EDTA }
\end{array}
$$

10x modified Gitschier buffer (MGB)

$$
\begin{array}{ll}
6.7 \mathrm{ml} & 1 \mathrm{M} \text { Tris- } \mathrm{HCl}, \mathrm{pH} 8.8 \\
1.66 \mathrm{ml} & \left.1 \mathrm{M} \mathrm{(NH}_{4}\right)_{2} \mathrm{SO}_{4} \\
650 \mu \mathrm{l} & 1 \mathrm{M} \mathrm{MgCl}_{2}
\end{array}
$$

Adjust final volume to $10 \mathrm{ml}$ with $\mathrm{H}_{2} \mathrm{O}$.

1x MGB buffer (Working solution)

$$
\begin{array}{ll}
1 \mathrm{ml} & 10 \times \text { MGB } \\
500 \mu \mathrm{l} & 10 \% \text { Triton X-100 } \\
8.4 \mathrm{ml} & \mathrm{H}_{2} \mathrm{O}
\end{array}
$$

Proteinase $\mathrm{K}$ (Stock $10 \mathrm{mg} / \mathrm{ml}$ in $\mathrm{ddH}_{2} \mathrm{O}$ )

$10 \mathrm{mM}$ dNTP (50x Stock)

$2.5 \mathrm{mM}$ each Nucleotide (dATP, dCTP, dGTP, dTTP) (Boehringer)

$200 \mu \mathrm{M}$ Final concentration per PCR-reaction (50 $\mu \mathrm{M}$ each Nucleotide)

\subsection{Protein biochemistry buffers and solutions}

Phosphatase inhibitors

1 Tablet PhosStop phosphatase inhibitor cocktail (Roche)

Added freshly to $10 \mathrm{ml}$ of protein lysis buffer before use. 
Protease inhibitors

1 Tablet Complete Mini protease inhibitor (Roche)

Added freshly to $10 \mathrm{ml}$ of protein lysis buffer before use.

Modified Ripa buffer (protein lysis buffer)

$50 \mathrm{mM} \quad$ Tris- $\mathrm{HCl}(\mathrm{pH} \mathrm{7.4)}$

$150 \mathrm{mM} \quad \mathrm{NaCl}$

$1.0 \mathrm{mM} \quad$ EDTA

$0,1 \% \quad$ SDS

$1,0 \% \quad$ Sodium deoxycholate

$1,0 \% \quad$ Triton $\mathrm{X}-100$

Phosphatase and protease inhibitors were added freshly to the buffer before use.

Sucrose homogenization buffer (protein lysis buffer)

$\begin{array}{ll}0.32 \mathrm{M} & \text { Sucrose } \\ 0.01 \mathrm{M} & \text { Tris- } \mathrm{HCl}(\mathrm{pH} \mathrm{7.4)} \\ 0.01 \mathrm{M} & \mathrm{NaHCO}_{3} \\ 0.01 \mathrm{M} & \mathrm{MgCl}_{2}\end{array}$

Phosphatase and protease inhibitors were added freshly to the buffer before use.

$0.32 \mathrm{M}$ Sucrose homogenization buffer (synaptosomal extraction buffer)
$0.32 \mathrm{M}$
Sucrose
$4.0 \mathrm{mM}$
HEPES/NaOH (pH 7.4)

Phosphatase and protease inhibitors were added freshly to the buffer before use. Steril filtered and stored at $4{ }^{\circ} \mathrm{C}$ up to 4 weeks.

0.8 M Sucrose gradient solution
$0.8 \mathrm{M}$
Sucrose
$4.0 \mathrm{mM}$
$\mathrm{HEPES} / \mathrm{NaOH}(\mathrm{pH} 7.4)$

Phosphatase and protease inhibitors were added freshly to the buffer before use. Steril filtered and stored at $4{ }^{\circ} \mathrm{C}$ up to 4 weeks.

\subsection{Sucrose gradient solution}

$$
\begin{array}{ll}
1.2 \mathrm{M} & \text { Sucrose } \\
4.0 \mathrm{mM} & \text { HEPES/NaOH }(\mathrm{pH} \mathrm{7.4})
\end{array}
$$

Phosphatase and protease inhibitors were added freshly to the buffer before use. Steril filtered and stored at $4{ }^{\circ} \mathrm{C}$ up to 4 weeks. 


\subsection{SDS PAGE and Western Blotting}

5x SDS sample buffer

$\begin{array}{ll}100 \mathrm{mM} & \text { Tris, pH } 6.8 \\ 5 \%(\mathrm{w} / \mathrm{v}) & \text { SDS } \\ 10 \%(\mathrm{v} / \mathrm{v}) & 0.4 \% \text { Bromphenolblue (in } \mathrm{EtOH}) \\ 50 \%(\mathrm{v} / \mathrm{v}) & \text { Glycerol }\end{array}$

4x LDS sample buffer (NuPAGE)

$\begin{array}{ll}4.0 \mathrm{ml} & 100 \% \text { Glycerol (f.c. } 40 \%) \\ 0.682 \mathrm{~g} & \text { Tris base (f.c. } 564 \mathrm{mM}) \\ 0.666 \mathrm{~g} & \text { Tris- } \mathrm{HCl} \text { (f.c. } 424 \mathrm{mM} \text { ) } \\ 0.8 \mathrm{~g} & \text { LDS (Lithium dodecyl sulfate) } \\ 250 \mu \mathrm{l} & 1 \% \text { Phenol red solution (f.c. } 2.5 \%(\mathrm{v} / \mathrm{v})) \\ 750 \mu \mathrm{l} & 1 \% \text { Serva Blue G250 solution (f.c. } 7.5 \%(\mathrm{v} / \mathrm{v})) \\ 0.006 \mathrm{~g} & \text { EDTA (f.c. } 2 \mathrm{mM})\end{array}$

Filled up to $10 \mathrm{ml}$ with $\mathrm{dd}_{2} \mathrm{O}$. $1 \mathrm{x}$ buffer had a $\mathrm{pH}$ of 8.5 . No $\mathrm{pH}$ adjustment necessary. For working solution added $20 \mu \mathrm{l}$ of $1 \mathrm{M}$ DTT to $50 \mathrm{ml} 4 \mathrm{x}$ LDS sample buffer.

$1 \mathrm{M}$ Dithiothreitol (DTT)

$$
1.5425 \mathrm{~g} \quad \text { DTT }
$$

Filled up to $10 \mathrm{ml}$ with $\mathrm{ddH}_{2} \mathrm{O}$.

4x Tris-HCl (Separation gel buffer)

$$
1.5 \mathrm{M} \quad \text { Tris- } \mathrm{HCl} \mathrm{pH} 8.8
$$

$4 x$ Tris- $\mathrm{HCl}$ (Stacking gel buffer)

$$
1.0 \mathrm{M} \quad \text { Tris- } \mathrm{HCl} \mathrm{pH} 6.8
$$

20x Tris buffered saline (TBS)
$1.0 \mathrm{M}$
Tris base
$3.0 \mathrm{M}$
$\mathrm{NaCl}$

Adjusted to $\mathrm{pH} 7.4$ with $37 \% \mathrm{HCl}$. 
1x TBS with Tween-20 (TBST)
$50 \mathrm{mM}$
Tris- $\mathrm{HCl}(\mathrm{pH} 7.4)$
$0.05 \%$
Tween-20

Blocking Buffer

$5 \% \quad$ Non-fat dry milk-powder in 1x TBST

Not stored longer than one week at $4{ }^{\circ} \mathrm{C}$.

Western blot stripping buffer
$0.2 \mathrm{M}$
Glycine- $\mathrm{HCl}(\mathrm{pH} 2.5)$
$0.1 \%$
Tween-20

Alternatively used $0.5 \mathrm{M} \mathrm{NaOH}$ solution to strip western blots.

$8.0 \%$ Polyacrylamide separating gel (4 gels of $0.75 \mathrm{~mm}$ thickness)
$9.3 \mathrm{ml}$
$\mathrm{ddH}_{2} \mathrm{O}$
$5.3 \mathrm{ml}$
$30 \%$ Acrylamide/bis-acrylamide (29:1) - Sigma
$5.0 \mathrm{ml}$
1.5 M Tris Separation gel buffer
$0.2 \mathrm{ml}$
$10 \%$ Sodium dodecyl sulfate (SDS)
$0.2 \mathrm{ml}$
$10 \%$ Ammonium persulphate (APS)
$0.012 \mathrm{ml}$
TEMED (Biorad)

$12 \%$ Polyacrylamide separating gel (4 gels of $0.75 \mathrm{~mm}$ thickness)
$6.6 \mathrm{ml}$
$\mathrm{ddH}_{2} \mathrm{O}$
$8.0 \mathrm{ml}$
$30 \%$ Acrylamide/bis-acrylamide (29:1) - Sigma
$5.0 \mathrm{ml}$
1.5 M Tris Separation gel buffer
$0.2 \mathrm{ml}$
$10 \%$ Sodium dodecyl sulfate (SDS)
$0.2 \mathrm{ml}$
$10 \%$ Ammonium persulphate (APS)
$0.008 \mathrm{ml}$
TEMED (Biorad)

$15 \%$ Polyacrylamide separating gel (4 gels of $0.75 \mathrm{~mm}$ thickness)
$4.6 \mathrm{ml}$
$\mathrm{dd}_{2} \mathrm{O}$
$10.0 \mathrm{ml}$
$30 \%$ Acrylamide/bis-acrylamide (29:1) - Sigma
$5.0 \mathrm{ml}$
1.5 M Tris Separation gel buffer
$0.2 \mathrm{ml}$
$10 \%$ Sodium dodecyl sulfate (SDS)
$0.2 \mathrm{ml}$
$10 \%$ Ammonium persulphate (APS)
$0.008 \mathrm{ml}$
TEMED (Biorad) 
Polyacrylamide stacking gel (4 gels of $0.75 \mathrm{~mm}$ thickness)

$5.5 \mathrm{ml}$

$\mathrm{ddH}_{2} \mathrm{O}$

$1.3 \mu \mathrm{l}$

$30 \%$ Acrylamide/bis-acrylamide (29:1) - Sigma

$1 \mathrm{ml}$

$1 \mathrm{M}$ Tris Stacking gel buffer

$0.08 \mathrm{ml}$

$10 \%$ Sodium dodecyl sulfate (SDS)

$0.08 \mathrm{ml}$

$10 \%$ Ammonium persulphate (APS)

$0.008 \mathrm{ml}$

TEMED (Biorad)

5x Trisglycine electrophoresis buffer
$125 \mathrm{mM}$
Tris base
$1.25 \mathrm{mM}$
Glycine
$0.5 \%$
Sodium dodecyl sulfate (SDS)

Working solution $(1 \mathrm{x})$

$200 \mathrm{ml}$

5x Trisglycine electrophoresis buffer

$800 \mathrm{ml}$

$\mathrm{ddH}_{2} \mathrm{O}$.

10x Trisglycine transfer buffer
$250 \mathrm{mM}$
Tris base
$1.92 \mathrm{M}$
Glycine

Working solution ( $1 \mathrm{x}$ with $20 \%$ methanol)

$100 \mathrm{ml}$ 10x Trisglycine transfer buffer

$200 \mathrm{ml}$ $100 \%$ Methanol

$700 \mathrm{ml}$

$\mathrm{ddH}_{2} \mathrm{O}$

Coomassie blue (Staining solution)

$2 \mathrm{~g}$

Coomassie brilliant blue (G-250)

11 $100 \%$ Methanol

$200 \mathrm{ml}$

Acetic acid

$800 \mathrm{ml}$

$\mathrm{ddH}_{2} \mathrm{O}$

Stirred overnight and filtered through Whatman paper.

Enhanced Chemiluminescence (ECL) Western-blot detection kit

Western Lightning ${ }^{\mathrm{TM}}$ Plus-ECL, Oxidizing reagent plus and Enhanced luminol reagent plus (Perkin Elmer Life Sciences, Inc.)

Roche PVDF Western Blotting Membrane pore size $0.2 \mu \mathrm{m}$ 


\subsection{DNA and Protein markers}

GeneRuler 1 kb DNA ladder

Fermentas

GeneRuler 100 bp DNA ladder

Fermentas

LabAid PageRuler Prestained Protein Ladder 10-170 kDa

Fermentas

\subsection{Immunohistochemistry buffers and solutions}

Avertin (Anesthetic)

$\begin{array}{ll}1 \mathrm{~g} & \text { Tribromethanol (2,2,2-Tribromethanol, 99\%) } \\ 0.81 \mathrm{ml} & \text { Amyl alcohol } \\ 71.49 \mathrm{ml} & \mathrm{ddH}_{2} \mathrm{O}\end{array}$

Heated $\mathrm{ddH}_{2} \mathrm{O}$ up to $40{ }^{\circ} \mathrm{C}$ and added to the tribromethanol and amylalcohol and stirred for $10 \mathrm{~min}$. Sterilefiltered, aliquotted and frozen at $-20^{\circ} \mathrm{C}$. Protected from light. Mice were intraperitoneal (IP) injected with $0.2 \mathrm{ml}$ per $10 \mathrm{~g}$ of body weight.

Phosphate buffer (Stock Solutions)
$0.2 \mathrm{M}$
Sodiumdihydrogenphosphate $\left(\mathrm{NaH}_{2} \mathrm{PO}_{4}\right)$
$0.2 \mathrm{M}$
Di-Sodiumhydrogenphosphate $\left(\mathrm{Na}_{2} \mathrm{HPO}_{4}\right)$

Working Solution ( $\mathrm{pH} 7.4)$

$\begin{array}{ll}20 \mathrm{ml} & 0.2 \mathrm{M} \text { Sodiumdihydrogenphosphate }\left(\mathrm{NaH}_{2} \mathrm{PO}_{4}\right) \\ 80 \mathrm{ml} & 0.2 \mathrm{M} \text { Di-Sodiumhydrogenphosphate }\left(\mathrm{Na}_{2} \mathrm{HPO}_{4}\right) \\ 100 \mathrm{ml} & \mathrm{ddH}_{2} \mathrm{O}\end{array}$

10x Phosphate buffered saline (PBS)

$\begin{array}{ll}1.7 \mathrm{M} & \mathrm{NaCl} \\ 34 \mathrm{mM} & \mathrm{KCl} \\ 40 \mathrm{mM} & \mathrm{Na}_{2} \mathrm{HPO}_{4}{ }^{\star} 2 \mathrm{H}_{2} \mathrm{O} \\ 18 \mathrm{mM} & \mathrm{K}_{2} \mathrm{HPO}_{4}\end{array}$

$\mathrm{pH}$ adjusted to 7.2 with $1 \mathrm{~N} \mathrm{NaOH}$.

1x PBS (Working solution)

$\begin{array}{ll}100 \mathrm{ml} & 10 x \mathrm{PBS} \\ 900 \mathrm{ml} & \mathrm{ddH}_{2} \mathrm{O}\end{array}$


Bovine Serum Albumin (PBS/BSA)

$\begin{array}{ll}20 \mathrm{ml} & 0.2 \mathrm{M} \text { Sodiumdihydrogenphosphate }\left(\mathrm{NaH}_{2} \mathrm{PO}_{4}\right) \\ 80 \mathrm{ml} & 0.2 \mathrm{M} \text { Di-Sodiumhydrogenphosphate }\left(\mathrm{Na}_{2} \mathrm{HPO}_{4}\right) \\ 1.8 \mathrm{~g} & \mathrm{NaCl} \\ 1.0 \mathrm{~g} & \text { Bovines Serum Albumin (BSA) } \\ 100 \mathrm{ml} & \mathrm{ddH}_{2} \mathrm{O}\end{array}$

16\% Paraformaldehyde (PFA, Stock solution)

$\begin{array}{ll}80 \mathrm{~g} & \text { Paraformaldehyde } \\ 450 \mathrm{ml} & \mathrm{dd}_{2} \mathrm{O}\end{array}$

Heated up to $65^{\circ} \mathrm{C}$ while stirring and then stirred for another 20 min. Droplets of $5 \mathrm{~N}$ $\mathrm{NaOH}$ added until solution turned clear. Filled up to a final volume of $500 \mathrm{ml}$ with $\mathrm{ddH}_{2} \mathrm{O}$ and left to cool down. Filtered through $500 \mathrm{ml}$ Nalgene sterile filter unit. Aliquotted and frozen at $-20^{\circ} \mathrm{C}$.

4\% Paraformaldehyde (PFA)

$\begin{array}{ll}100 \mathrm{ml} & 0.2 \mathrm{M} \text { Sodiumdihydrogenphosphate }\left(\mathrm{NaH}_{2} \mathrm{PO}_{4}\right) \\ 400 \mathrm{ml} & 0.2 \mathrm{M} \text { Di-Sodiumhydrogenphosphate }\left(\mathrm{Na}_{2} \mathrm{HPO}_{4}\right) \\ 250 \mathrm{ml} & 16 \% \text { PFA } \\ 8.0 \mathrm{~g} & \mathrm{NaCl} \\ 250 \mathrm{ml} & \mathrm{ddH}_{2} \mathrm{O}\end{array}$

Citrate Buffer (Stock Solutions)
$0.1 \mathrm{M}$
Citric acid $\left(\mathrm{C}_{6} \mathrm{H}_{8} \mathrm{O}_{7}{ }^{*} \mathrm{H}_{2} \mathrm{O}\right)$
$0.1 \mathrm{M}$
Sodium citrate $\left(\mathrm{C}_{6} \mathrm{H}_{5} \mathrm{O}_{7} \mathrm{Na}_{3}{ }^{*} 2 \mathrm{H}_{2} \mathrm{O}\right)$

Stored at $4^{\circ} \mathrm{C}$.

Working Solution (0.01 M pH 6.0)
$9.0 \mathrm{ml}$
$0.1 \mathrm{M}$ Citric acid $\left(\mathrm{C}_{6} \mathrm{H}_{8} \mathrm{O}_{7}{ }^{*} \mathrm{H}_{2} \mathrm{O}\right)$
$41 \mathrm{ml}$
$0.1 \mathrm{M}$ Sodium citrate $\left(\mathrm{C}_{6} \mathrm{H}_{5} \mathrm{O}_{7} \mathrm{Na}_{3}{ }^{*} 2 \mathrm{H}_{2} \mathrm{O}\right)$
$450 \mathrm{ml}$ $\mathrm{ddH}_{2} \mathrm{O}$

Always prepared freshly.

Tris-buffer (Stock Solution)
$0.5 \mathrm{M}$
Tris base

$\mathrm{pH}$ adjusted to 7.6 with $37 \% \mathrm{HCl}$. Stored at $4{ }^{\circ} \mathrm{C}$. 
Working Solution

$\begin{array}{ll}100 \mathrm{ml} & 0.5 \mathrm{M} \text { Tris base }(\mathrm{pH} \mathrm{7.6}) \\ 9.0 \mathrm{~g} & \mathrm{NaCl}\end{array}$

Filled up to a final volume of $1000 \mathrm{ml}$ with $\mathrm{dd}_{2} \mathrm{O}$. Always prepared freshly.

2\% Milk-powder in Tris-buffer

$20 \mathrm{~g} \quad$ Non-fat dry milk-powder

Filled up to a final volume of $1000 \mathrm{ml}$ with Tris-buffer working solution.

\subsection{Buffers for immunohistochemisty of vibrotome and cryo sections}

$0.1 \%$ Triton X-100 in $1 \times$ PBS

$\begin{array}{ll}40 \mu \mathrm{l} & 4 \% \text { Triton X-100 } \\ 960 \mu \mathrm{l} & 1 \mathrm{x} \text { PBS }\end{array}$

$4 \%$ Horse-Serum, $0.1 \%$ Triton X-100 in 1x PBS

$40 \mu \mathrm{l} \quad$ Horse Serum

$960 \mu \mathrm{l} \quad 0.1 \%$ Triton X-100 in $1 \mathrm{x}$ PBS

$1 \%$ Horse-Serum, $0.1 \%$ Triton X-100 in 1x PBS

$10 \mu \mathrm{l} \quad$ Horse Serum

$990 \mu \mathrm{l} \quad 0.1 \%$ Triton X-100 in $1 \times$ PBS

$1.5 \%$ Horse Serum in $1 \times$ PBS

$15 \mu \mathrm{l} \quad$ Horse Serum

$985 \mu \mathrm{l} \quad 1 \times$ PBS

\subsection{Mounting media}

$\begin{array}{ll}\text { Eukit } & \text { (Kindler) } \\ \text { Aqua polymount } & \text { (Polyscience) } \\ \text { Shandon Cryomatrix } & \text { (Thermo Scientific) }\end{array}$




\subsection{Histological stains and reagents}

Mayer's Haematoxylin solution

$1 \mathrm{~g} \quad$ Haematoxylin

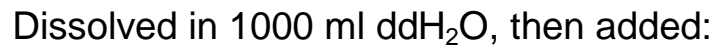

$0.2 \mathrm{~g} \quad$ Sodium iodate

$50 \mathrm{~g} \quad$ Potassium aluminium sulphate

under constant shaking. Then added:

$50 \mathrm{~g} \quad$ Chloralhydrate

$1 \mathrm{~g} \quad$ Citric acid

Filtered before use.

Eosin Stock solution (10x)

$10 \mathrm{~g} \quad$ Eosin

Dissolved in $100 \mathrm{ml}$ of $\mathrm{ddH}_{2} \mathrm{O}$ and left to mature.

Eosin working solution
$2.5 \mathrm{ml}$
Eosin Stock solution

Filled up to $250 \mathrm{ml}$ with $\mathrm{ddH}_{2} \mathrm{O}$ and finished by adding 12 drops of glacial acetic acid.

Scott's solution

$\begin{array}{ll}2 \mathrm{~g} & \mathrm{KHCO}_{3} \\ 20 \mathrm{~g} & \mathrm{MgSO}_{4}\end{array}$

Filled up to a final volume of $1000 \mathrm{ml}$ with $\mathrm{ddH}_{2} \mathrm{O}$.

$\mathrm{HCl}-A l c o h o l$

$1.25 \mathrm{ml} \quad$ Conc. $\mathrm{HCl}$

$350 \mathrm{ml} \quad 100 \%$ Ethanol

$150 \mathrm{ml} \quad \mathrm{ddH}_{2} \mathrm{O}$

$\beta$-Gal Staining buffer (stock solutions)

$\begin{array}{ll}500 \mathrm{mM} & \text { Potassium ferricyanid (in PBS) } \\ 500 \mathrm{mM} & \text { Potassium ferrocyanid (in PBS) } \\ 2 \mathrm{mM} & \mathrm{MgCl}_{2} \\ 20 \mathrm{mg} / \mathrm{ml} & \text { X-Gal (in DMSO) }\end{array}$


Working solution

$\begin{array}{ll}5 \mathrm{mM} & \text { Potassium ferricyanid } \\ 5 \mathrm{mM} & \text { Potassium ferrocyanid } \\ 2 \mathrm{mM} & \mathrm{MgCl}_{2} \\ 600 \mu \mathrm{l} & \text { X-Gal (f.c. } 1.2 \mathrm{mg} / \mathrm{ml} \text { ) }\end{array}$

Filled up to a final volume of $10 \mathrm{ml}$ with $1 \mathrm{x}$ PBS.

\subsection{Oligonucleotids}

All oligonucleotides (primers) were synthesized at "Oligo Syntesys Lab" of the Max Planck Institute of Experimental Medicine. They were provided at $50 \mathrm{pM}$ stock concentrations. In house identification numbers are displayed in brackets.

\subsection{Genotyping primer}

CKII-Cre

In house No.

Forward: 5'-CGAGTGGCCCCTAGTTCTGGGGGCAGC-3'

(7964)

Reverse: 5'-CGTTGCATCGACCGGTAATGCAGGC-3'

Emx1-Cre

Forward: 5'-CAGGGTGTTATAAGCAATCCC-3'

Reverse: 5'-CCTGGAAAATGCTTCTGTCCG-3'

$\operatorname{Nrg} 1^{f / f}$

Forward: 5'-TCCTTTTGTGTGTGTTCAGCACCGG-3'

Reverse: 5'-GCACCAAGTGGTTGCGATTGTTGCT-3'

NEX-Cre and NEX-CreERT2

Forward: 5'-GAGTCCTGGAATCAGTCTTTTTC-3'

Reverse: 5'-CCGCATAACCAGTGAAACAG-3'

Reverse: 5'-AGAATGTGGAGTAGGGTGAC-3'

PV-GFP

Forward: 5'-GACACTGCAGCGCTGGTCAT-3'

Reverse: 5'-CCAGCTTGTGCCCCAGGATGT-3' 
R26R-floxtdTomato

Forward: 5'-TACGGCATGGACGAGCTGTACAAGTAA-3'

Reverse: 5'-CAGGCGAGCAGCCAAGGAAA-3'

R26R-floxLacZ

Forward: 5'-AAAGTCGCTCTGAGTTGTTAT -3'

Reverse: 5'-GCGAAGAGTTTGTCCTCAACC -3'

Reverse: 5'-GGAGCGGGAGAAATGGATATG -3'

Stop-Nrg1

Forward: 5'-GTCCACAAATACCCACTTTAGGCCAGC-3'

Reverse: 5'-GGTGGCTATAAAGAGGTCATCAG-3'

Thy1.2-driven Nrg1-tg, HA-Nrg1-tg and HA-Nrg1 ${ }^{\text {GIEF }}$

Forward: 5'-GTCCACAAATACCCACTTTAGGCCAGC-3'

Reverse: 5'-GGCTTTCTCTGAGTGGCAAAGGACC-3'

\subsection{Enzymes}

REDTaq DNA Polymerase Sigma-Aldrich

GoTaq Polymerase Promega

\subsection{Antibodies}

\subsubsection{Primary Antibodies}

\begin{tabular}{|c|c|c|c|c|c|}
\hline Antibody & Cat. No. & Species & Company & Dilution & Purpose \\
\hline Actin & MAB1501 & mono-ms & Millipore & 1000 & WB \\
\hline AKT & $\# 9272$ & poly-rb & Cell Signaling & 5000 & WB \\
\hline AKT & $\# 4691$ & poly-rb & Cell Signaling & 5000 & WB \\
\hline $\begin{array}{c}\text { phospho-AKT (Ser473) } \\
\text { (D9E) }\end{array}$ & $\# 4060$ & poly-rb & Cell Signaling & 5000 & WB \\
\hline $\begin{array}{c}\text { phospho-AKT (Ser473) } \\
(736 E 11)\end{array}$ & $\# 3787$ & poly-rb & Cell Signaling & 5000 & WB \\
\hline Cofilin & $\# 3312$ & poly-rb & Cell Signaling & 1000 & WB \\
\hline $\begin{array}{c}\text { phospho-Cofilin 1 (hSer 3)- } \\
\text { R }\end{array}$ & sc-12912-R & poly-rb & Santa Cruz & 1000 & WB \\
\hline Cre & PRB-106C & poly-rb & Covance & 1000 & IHC \\
\hline
\end{tabular}


Materials

\begin{tabular}{|c|c|c|c|c|c|}
\hline Antibody & Cat. No. & Species & Company & Dilution & Purpose \\
\hline ErbB4 & mAb10 & mono-rb & A. Buonanno & $1 \mu \mathrm{g} / \mathrm{ml}$ & WB \\
\hline ErbB4 (E200) & ab32375 & mono-rb & abcam & 1000 & WB \\
\hline GAD67 & MAB5406 & mono-ms & Chemicon & 100 & $\mathrm{IHC}$ \\
\hline GFAP & Z 0334 & poly-rb & Dako & 500 & $\mathrm{IHC}$ \\
\hline GFP & $\begin{array}{l}600-101- \\
215 \\
\end{array}$ & $\begin{array}{l}\text { mono- } \\
\text { goat }\end{array}$ & Rockland & 500 & $\mathrm{IHC}$ \\
\hline GLT-1 & AB1783 & poly-gp & Chemicon & 20000 & WB \\
\hline $\mathrm{HA}$ & ab9110 & poly-rb & abcam & 500 & $\mathrm{IHC}, \mathrm{WB}$ \\
\hline HA.11 & MMS-101P & mono-ms & Covance & 250 & $\mathrm{IHC}, \mathrm{WB}$ \\
\hline IBA1 & $019-19741$ & poly-rb & Wako & 700 & $\mathrm{IHC}$ \\
\hline Krox-20 & PRB-236P & poly-rb & Covance & 100 & $\mathrm{IHC}$ \\
\hline LIMK1 & \#3842 & poly-rb & Cell Signaling & 1000 & WB \\
\hline phospho-LIMK1 (Thr508) & ab38508 & poly-rb & abcam & 500 & WB \\
\hline $\begin{array}{c}\text { phospho-LIMK1 } \\
\text { (Thr508)/LIMK2 (Thr505) }\end{array}$ & \#3841 & poly-rb & Cell Signaling & 1000 & WB \\
\hline MBP & A0623 & poly-rb & Dako & 500 & WB \\
\hline NeuN & MAB377 & mono-ms & Chemicon & 100 & $\mathrm{IHC}$ \\
\hline Neuregulin-1a/b1/2 (C-20) & SC-348 & poly-rb & Santa Cruz & $100 / 500$ & $\mathrm{IHC}, \mathrm{WB}$ \\
\hline Neurogranin & AB1763 & poly-rb & Chemicon & 1000 & $\mathrm{IHC}$ \\
\hline Olig2 & DF308 & poly-rb & $\begin{array}{l}\text { John Alberta, } \\
\text { Harvard }\end{array}$ & 200 & $\mathrm{IHC}$ \\
\hline $\begin{array}{c}\mathrm{p} \text {-HER4/ErbB4 (Tyr1284) } \\
(21 \mathrm{~A} 9)\end{array}$ & \#4757 & mono-rb & Cell Signaling & 1000 & WB \\
\hline $\begin{array}{c}\text { p44/42 MAPK (Erk1/2) } \\
\text { (137F5) }\end{array}$ & \#4695 & poly-rb & Cell Signaling & 5000 & WB \\
\hline $\begin{array}{c}\text { phospho-p44/42 MAPK } \\
\text { (Erk1/2) (Thr202/Tyr204) } \\
(20 G 11) \\
\end{array}$ & \#4376 & poly-rb & Cell Signaling & 5000 & WB \\
\hline Parvalbumin 28 & PV-28 & poly-rb & Swant & 200 & $\mathrm{IHC}$ \\
\hline Synaptophysin & 101011 & mono-ms & $\begin{array}{l}\text { Synaptic } \\
\text { Systems }\end{array}$ & 1000 & WB \\
\hline Tubulin & T 5168 & mono-ms & Sigma & 1000000 & WB \\
\hline
\end{tabular}

\subsubsection{Secondary Antibodies}

$\alpha$-goat-Alexa488

$\alpha$-guinea pig-cy3

$\alpha$-guinea pig-cy5

$\alpha$-guinea pig-HRP

$\begin{array}{llll}\text { Species } & \text { Company } & \text { Dilution } & \text { Purpose } \\ \text { donkey } & \text { Dianova } & 1: 500 & \text { IHC } \\ \text { goat } & \text { Dianova } & 1: 500 & \text { IHC } \\ \text { goat } & \text { Dianova } & 1: 500 & \text { IHC } \\ \text { goat } & \text { Dianova } & 1: 5000 & \text { WB }\end{array}$




$\begin{array}{lllll}\alpha \text {-mouse-AF555 } & \text { donkey } & \text { Dianova } & 1: 1000 & \text { IHC } \\ \alpha \text {-mouse-Biotin } & \text { goat } & \text { Acris } & 1: 100 & \text { IHC } \\ \alpha \text {-mouse-cy2 } & \text { goat } & \text { Dianova } & 1: 100 & \text { IHC } \\ \alpha \text {-mouse-cy3 } & \text { goat } & \text { Dianova } & 1: 1000 & \text { IHC } \\ \alpha \text {-mouse-cy5 } & \text { donkey } & \text { Dianova } & 1: 500 & \text { IHC } \\ \alpha \text {-mouse-DL488 } & \text { goat } & \text { Dianova } & 1: 100 & \text { IHC } \\ \alpha \text {-mouse-DL633 } & \text { goat } & \text { Dianova } & 1: 250 & \text { IHC } \\ \alpha \text {-mouse-HRP } & \text { goat } & \text { Dianova } & 1: 5000 & \text { WB } \\ \alpha \text {-rabbit-Alexa488 } & \text { goat } & \text { Dianova } & 1: 100 & \text { IHC } \\ \alpha \text {-rabbit-Alexa555 } & \text { donkey } & \text { Dianova } & 1: 500 & \text { IHC } \\ \alpha \text {-rabbit-Biotin } & \text { goat } & \text { Novus-Bio } & 1: 100 & \text { IHC } \\ \alpha \text {-rabbit-cy2 } & \text { goat } & \text { Dianova } & 1: 100 & \text { IHC } \\ \alpha \text {-rabbit-cy3 } & \text { goat } & \text { Dianova } & 1: 1000 & \text { IHC } \\ \alpha \text {-rabbit-cy5 } & \text { donkey } & \text { Dianova } & 1: 500 & \text { IHC } \\ \alpha \text {-rabbit-DL488 } & \text { goat } & \text { Dianova } & 1: 100 & \text { IHC } \\ \alpha \text {-rabbit-HRP } & \text { goat } & \text { Promega } & 1: 5000 & \text { WB }\end{array}$

\subsection{Mouse lines}

Stop-Nrg1

conditional $\beta$-actin eGFP-Stop-flox 2HA-Nrg1 type III $\beta 1$ a transgenic mouse (Unterbarnscheidt et al., in preparation)

Nrg1-tg

Thy1.2 promtor-driven Nrg1 type III $\beta 1$ a transgenic mouse

(Wolpowitz et al., 2000)

HA-Nrg1-tg

Thy1.2 2HA-Nrg1 type III $\beta 1$ a transgenic mouse

(Velanac et al., 2011)

HA-Nrg $1^{\text {GIEF }}$

Thy1.2 2HA-Nrg1 ${ }^{\text {GIEF }}$ transgenic mouse

(Velanac et al., 2011)

$\mathrm{Nrg}^{1 / / 4}$

Conditional floxed NRG1 knockout mouse (Li et al., 2002) 
NEX-Cre

Cre driver mouse line, generated by homologous recombination of Cre into the NEX locus (Goebbels et al., 2006)

\section{NEX-CreERT2}

Cre driver mouse line, generated by homologous recombination of Tamoxifen inducible CreERT2 into the NEX locus (Agarwal et al., 2011)

CKII-Cre

Cre driver mouse line, expressing Cre as a transgene under control of

CamKIla promoter (Minichiello et al., 1999)

\section{Emx1-Cre}

Cre driver mouse line, generated by homologous recombination of IREScre into the exon encoding the 3' untranslated region of the mouse Emx1 gene (Gorski et al., 2002)

\section{R26R-floxtdTomato}

Rosa26 knock-in floxed tdTomato Cre-reporter mouse line

(Madisen et al., 2010)

R26R-floxLacZ

Rosa26 knock-in floxed LacZ Cre-reporter mouse line

(Soriano, 1999)

PV-GFP

Bac-transgenic mouse expression eGFP under regulatory elements of the parvalbumin gene (Meyer et al., 2002) 


\section{Methods}

\subsection{Animal maintenance, handling and treatment}

\subsubsection{Generation of conditional Stop-Nrg1 transgenic mice}

Stop-Nrg1 mice were generated by cloning the HA-NRG1 ${ }^{\mathrm{FL}} \mathrm{cDNA}$ used for the generation of HA-Nrg1-tg mice (Velanac et al., 2011) via Spel and Xhol restriction digest into the polylinker of the $\beta$-actin Stop-eGFP-flox cassette in a pBluescriptKS vector. The vector backbone harbored a fragment of a chicken $\beta$-actin promoter, an eGFP-Stop cassette flanked by two loxP sites, and a bovin growth hormone polyA site. The transgene cassette was excised from the plasmid via Sall and injected into C57bl6n oocytes. Initial cloning of the vector backbone was performed by Maike Gummert and HA-NRG1 ${ }^{\mathrm{FL}} \mathrm{cDNA}$ insertion and linearization was conducted by Viktorija Velanac at the Max-Planck-Institute of Experimental Medicine in Göttingen. One positive founder was born. The transgenic line was maintained on a C57/BI6N background.

\subsubsection{Breeding of mouse mutants}

Mice mutants were bred starting at 8 weeks of age. To maintain and expand the mutant lines, mutant mice were bred to $W T$ mice of the C57/BI6N strain.

\subsubsection{Animal maintenance and handling}

All mouse mutants used in this study were maintained and bred in the animal facility of the Max-Planck-Institute of Experimental Medicine. Animals were kept and handled in compliance with animal policies of the Max-Planck-Institute of Experimental Medicine and approved by the German Federal State of Lower Saxony.

\subsubsection{Tamoxifen injection}

For the acute activation of NRG1 overexpression, the NEX-CreERT2 mouse line was used. The CreERT2 sequence in this mouse line is an improved version of the original tamoxifen-inducible Cre recombinase (Feil et al., 1997; Metzger and Chambon, 2001), which is fused to a mutated human oestrogen receptor (ER) ligand-binding domain (LBD) (Feil et al., 1997). In the absence of tamoxifen, a synthetic ligand of the ER, the CreERT2 variant is located in the cytoplasm and is inactive. Addition of tamoxifen induces nuclear transfer and site-specific recombination of loxP-flanked genomic DNA (Feil et al., 1997). Tamoxifen was administered to adult mice at different ages by intraperitoneal injection (IP) or 
postnatally by IP injection of the mother and transmission of tamoxifen via milk. Concentrations used ranged from 25 to $100 \mathrm{mg} / \mathrm{kg}$ of body weight and from 2 to 10 consecutive days of injection (full induction $=10$ days of $100 \mathrm{mg} / \mathrm{kg}$ body weight).

\subsubsection{Phenotyping of tail biopsies of Stop-Nrg1 mice}

For routine genotyping of Stop-Nrg1 mice the fluorescence of the loxP sites-flanked eGFP-Stop-cassette of the transgene cassette was used. In unrecombined cells, e.g. in the tailbiopsie it can be used to genotype mice. For fluorescent phenotyping of transgenic mice, tail biopsies were examined under fluorescent light of $488 \mathrm{~nm}$ excitation under a fluorescent microscope (Leica), prior to DNA extraction and genotyping for Cre recombinase transgenes by PCR (see section: Preparation of mouse genomic DNA, below).

\subsection{Preparation of mouse genomic DNA}

\subsubsection{Standard method of genomic DNA isolation}

Routine genotyping of transgenic mouse lines was performed on genomic DNA isolated from $5 \mathrm{~mm}$ pieces of tail biopsies. Each tail was lysed in a tightly closed tube with $200 \mu \mathrm{l}$ of lysis buffer, that contained $180 \mu \mathrm{l}$ of 1 x MGB (working solution) and 20 $\mu \mathrm{l}$ of proteinase $\mathrm{K}(10 \mathrm{mg} / \mathrm{ml})$. Lysis was carried out over night $(\mathrm{ON})$ at $52{ }^{\circ} \mathrm{C}$ and vigorous shaking. Digested tails where centrifuged $5 \mathrm{~min}$ at $5000 \mathrm{rpm}$ followed by heat inactivation of the proteinase $\mathrm{K}$ at $95^{\circ} \mathrm{C}$ for $10 \mathrm{~min}$. Lysates were then mixed by shaking and centrifuged at $5000 \mathrm{rpm}$ for $10 \mathrm{~min}$ at room temperature (RT). $1 \mu \mathrm{l}$ of supernatant was used in genotyping PCR reactions (see below). Alternatively surface phase of the supernatant was diluted with $\mathrm{ddH}_{2} \mathrm{O}(1: 5)$ for increased purity of PCR results.

\subsection{2 nexttec ${ }^{\mathrm{TM}}$ Tissue \& Cells kit-based genomic DNA isolation}

The standard method was used to isolate genomic DNA from mouse tail biopsies in the beginning of this study. Meanwhile, a kit-based isolation method was aquired and established in the lab. Routine genomic DNA extraction was from then on performed by using nexttec ${ }^{\mathrm{TM}}$ 's $1^{\text {-Step }}$ Tissue \& Cells kit. The DNA isolation was performed according to the manufactures protocol. Briefly, tail biopsies were lysed in $300 \mu \mathrm{l}$ lysis buffer, containing $265 \mu \mathrm{l}$ of buffer $\mathrm{G} 1,10 \mu \mathrm{l}$ buffer $\mathrm{G} 2$ and $25 \mu \mathrm{l}$ buffer G3, by vigorous shaking for $30 \mathrm{~min}$ at $12.000 \mathrm{rpm}$ and $62{ }^{\circ} \mathrm{C}$. Meanwhile nexttec ${ }^{\mathrm{TM}}$ cleanPlate96 were equilibrated with $350 \mu$ of prep buffer for 5 min at RT and 
centrifuged for $1 \mathrm{~min}$ at $350 \mathrm{xg} .120 \mu \mathrm{l}$ of the tail lysate were then loaded onto columns and incubated for $3 \mathrm{~min}$ at RT before eluation by centrifugation at $750 \mathrm{xg}$ for 1 min into fresh tail tubes. Lysate were diluted 1:5 in $d_{d d H_{2}} \mathrm{O} .1 \mu$ of the lysate was used in genotyping PCR reactions (see below).

\subsection{DNA modification and analysis}

\subsubsection{DNA amplification in vitro by polymerase chain reaction (PCR)}

The polymerase chain reaction (PCR), originally reported by (Mullis et al., 1986), is a standard method that enables in vitro enzymatic amplification of selected DNA sequence. The reaction requires a thermostable DNA polymerase from the termophilic bacterium Thermus aquaticus (Taq Polymerase), that is stable at the melting temperature of the double stranded DNA and has an optimal activity at 72 ${ }^{\circ} \mathrm{C}$. In the reaction a template sequence for amplification is defined by primers, single stranded oligonucleotides that complementary bind to the sense and antisense strands on the opposite ends of the template. The DNA amplification is carried out in a thermocycler (Thermocycler T3, Biometra) through multiple repetitions of three step PCR cycles. In the first step of the PCR reaction the double stranded DNA template is broken up at $95^{\circ} \mathrm{C}$. In the second step the primers anneal to the complementary sequences of the template DNA at their specific annealing temperature. In the last step each strand of the template is replicated by elongation of the primer sequence by the Taq Polymerase, which adds nucleotides complementary to the template.

In this study standard PCR master mix were setup with RedTaq polymerase (Sigma) as follows:

$\begin{array}{ll}1 \mu \mathrm{l} & \text { DNA }(100 \mathrm{pg}-100 \mathrm{ng}) \\ 1 \mu \mathrm{l} & \text { sense primer }(10 \mathrm{pM}) \\ 1 \mu \mathrm{l} & \text { antisense primer }(10 \mathrm{pM}) \\ 2 \mu \mathrm{l} & \text { dNTP mix }(2 \mathrm{mM}) \\ 2 \mu \mathrm{l} & 10 x \text { RedTaq buffer } \\ 1 \mu \mathrm{l} & \text { RedTaq polymerase }(1 \mathrm{U} / \mu \mathrm{l}) \\ 12 \mu \mathrm{l} & \text { ddH2O }\end{array}$

PCR products were then separated on agarose gels for visualization.

\subsubsection{Primer design}

All primers were designed manually, based on the template sequence information using the DNAStar (EditSeq) software, BLAST at NCBI, and oligocalculator 
(http://mbcf.dfci.harvard.edu/docs/oligocalc.html). They where designed to have at least 20 bp overlap with the template sequence, and a melting temperature of $\sim 60$ ${ }^{\circ} \mathrm{C}$. Oligonucleotides were synthesized in-house at the 'Oligo Synthesis Lab' of the Max-Planck-Institute of Experimental Medicine at a stock concentration of $50 \mathrm{pM}$ and diluted 1:5 in $\mathrm{dd}_{2} \mathrm{O}$ before use.

\subsubsection{Agarose gel electorphoresis for the size separation of DNA fragments}

To separate DNA fragments, resulting from genotyping PCRs, based on their size agarose gel electrophoresis was used (Voytas, 2001). For the separation, gels of 1 $\%-2 \%$ agarose in $1 x$ TAE buffer were used. For DNA visualization 2 to $3 \mu$ of $10 x$ GelRED $^{\mathrm{TM}}$ (Biotium) were added to $20 \mu \mathrm{l}$ DNA sample. Gels were placed into the electrophoresis chambers and filled with $1 \mathrm{x}$ TAE buffer until the gel surface was covered by buffer and the wells were filled. Standard DNA marker (100 bp or $1 \mathrm{~kb}$ ladder) and DNA samples (containing glycerol and tracking dye) were loaded into the wells. The chamber was then connected to a power supply and voltage was set to 70 $\mathrm{V}$ for the first run-in of the samples. Negatively charged DNA migrates to the positively charged cathode and smaller DNA fragments migrate faster through the gel than bigger fragments. When probes had successfully entered the gel, the voltage was increased to $100 \mathrm{~V}$. The gel was run until the desired fragment separation was achieved. For documentation, snapshots of UV-trans-illuminated gels were taken.

\subsubsection{Genotyping of mouse mutants}

At 3 weeks of age a tail biopsy was taken from mutant mice. DNA for genotyping was prepared with either the standard procedure or the nexttec ${ }^{\mathrm{TM}}$ Tissue \& Cells kit. The genotype was determined by performing genotyping PCRs with mutant specific genotyping primers described in the material section (Section 5.13). An example for a PCR master mix is given in the section above. PCRs were incubated in the thermocycler with following programs. 
6.3.5 PCR programs:

Stop-Nrg1

1. $95^{\circ} \mathrm{C} \quad 5 \mathrm{~min}$

2. $95^{\circ} \mathrm{C} \quad 45 \mathrm{sec}$

3. $56^{\circ} \mathrm{C} \quad 30 \mathrm{sec}$

4. $72{ }^{\circ} \mathrm{C} \quad 1 \mathrm{~min}$

$\rightarrow 2$ to 4 for 36 cycles

5. $56^{\circ} \mathrm{C} \quad 1 \mathrm{~min}$

6. $72{ }^{\circ} \mathrm{C} \quad 10 \mathrm{~min}$

7. $4^{\circ} \mathrm{C}$ pause

Nrg1-tg, HA-Nrg1-tg and HA-Nrg $1^{\text {GIEF }}$
1. $95^{\circ} \mathrm{C}$
$3 \min$
2. $95^{\circ} \mathrm{C}$
$45 \mathrm{sec}$
3. $58^{\circ} \mathrm{C}$
$30 \mathrm{sec}$
4. $72^{\circ} \mathrm{C}$
$1 \mathrm{~min}$
$\rightarrow 2$ to 4 for 35 cycles
5. $72{ }^{\circ} \mathrm{C}$
$10 \mathrm{~min}$
$6.4{ }^{\circ} \mathrm{C}$ pause

$\operatorname{Nrg} 1^{f / f}$
1. $94^{\circ} \mathrm{C} \quad 3 \mathrm{~min}$
2. $60^{\circ} \mathrm{C} \quad 30 \mathrm{sec}$
3. $72{ }^{\circ} \mathrm{C} \quad 50 \mathrm{sec}$
4. $94^{\circ} \mathrm{C} \quad 45 \mathrm{sec}$
$\rightarrow 2$ to 4 for 39 cycles
5. $60^{\circ} \mathrm{C} \quad 1 \mathrm{~min}$
6. $72{ }^{\circ} \mathrm{C} \quad 10 \mathrm{~min}$
7. $4^{\circ} \mathrm{C}$ pause

\section{NEX-Cre and NEX-CreERT2}

1. $94^{\circ} \mathrm{C} \quad 3 \mathrm{~min}$

2. $54^{\circ} \mathrm{C} \quad 30 \mathrm{sec}$

3. $72^{\circ} \mathrm{C} \quad 1 \mathrm{~min}$

4. $94^{\circ} \mathrm{C} \quad 30 \mathrm{sec}$

$\rightarrow 2$ to 4 for 39 cycles

$5.54^{\circ} \mathrm{C} \quad 1 \mathrm{~min}$

6. $72^{\circ} \mathrm{C} \quad 5 \mathrm{~min}$

7. $4^{\circ} \mathrm{C}$ pause 
CKII-Cre

1. $95^{\circ} \mathrm{C} \quad 3 \mathrm{~min}$

2. $94^{\circ} \mathrm{C} \quad 30 \mathrm{sec}$

3. $58^{\circ} \mathrm{C} \quad 30 \mathrm{sec}$

4. $72{ }^{\circ} \mathrm{C} \quad 30 \mathrm{sec}$

$\rightarrow 2$ to 4 for 35 cycles

$5.58^{\circ} \mathrm{C} \quad 1 \mathrm{~min}$

6. $72^{\circ} \mathrm{C} \quad 5 \mathrm{~min}$

7. $4^{\circ} \mathrm{C}$ pause

\section{Emx1-Cre}

1. $94^{\circ} \mathrm{C} \quad 3 \mathrm{~min}$

2. $55^{\circ} \mathrm{C} \quad 30 \mathrm{sec}$

3. $72{ }^{\circ} \mathrm{C} \quad 30 \mathrm{sec}$

4. $94^{\circ} \mathrm{C} \quad 30 \mathrm{sec}$

$\rightarrow 2$ to 4 for 35 cycles

5. $55^{\circ} \mathrm{C} \quad 1 \mathrm{~min}$

6. $72{ }^{\circ} \mathrm{C} \quad 5 \mathrm{~min}$

7. $4{ }^{\circ} \mathrm{C}$ pause

\section{R26R-floxtdTomato}

1. $95^{\circ} \mathrm{C} \quad 3 \mathrm{~min}$

2. $60^{\circ} \mathrm{C} \quad 30 \mathrm{sec}$

3. $72^{\circ} \mathrm{C} \quad 1.5 \mathrm{~min}$

4. $95^{\circ} \mathrm{C} \quad 30 \mathrm{sec}$

$\rightarrow 2$ to 4 for 45 cycles

5. $60^{\circ} \mathrm{C} \quad 1 \mathrm{~min}$

6. $72^{\circ} \mathrm{C} \quad 10 \mathrm{~min}$

7. $4^{\circ} \mathrm{C}$ pause

\section{R26R-floxLacZ}

1. $95^{\circ} \mathrm{C} \quad 3 \mathrm{~min}$

2. $56^{\circ} \mathrm{C} \quad 30 \mathrm{sec}$

3. $72^{\circ} \mathrm{C} \quad 1 \mathrm{~min}$

4. $95^{\circ} \mathrm{C} \quad 30 \mathrm{sec}$

$\rightarrow 2$ to 4 for 36 cycles

5. $56^{\circ} \mathrm{C} \quad 1 \mathrm{~min}$

6. $72{ }^{\circ} \mathrm{C} \quad 10 \mathrm{~min}$

7. $4^{\circ} \mathrm{C}$ pause 


$\boldsymbol{P V}$-GFP
$\begin{array}{ll}1.95^{\circ} \mathrm{C} & 3 \mathrm{~min} \\ 2.57^{\circ} \mathrm{C} & 40 \mathrm{sec} \\ 3.72^{\circ} \mathrm{C} & 45 \mathrm{sec} \\ 4.95^{\circ} \mathrm{C} & 30 \mathrm{sec} \\ \rightarrow & 2 \text { to } 4 \text { for } 40 \text { cycles } \\ 5.56{ }^{\circ} \mathrm{C} & 1 \mathrm{~min} \\ 6.72{ }^{\circ} \mathrm{C} & 10 \mathrm{~min} \\ 7.4{ }^{\circ} \mathrm{C} \text { pause }\end{array}$

\subsection{Protein biochemistry methods}

\subsubsection{Radioimmunoprecipitation assay (RIPA) buffer protein extraction}

For the analysis of proteins of mouse nervous system tissue, brains of mice at different ages were dissected and cortices (Cx), prefrontal cortices (PFC), hippocampi (Hipp) and thalamus (Thal) were micro-dissected on ice and afterwards frozen on dry ice and stored at $-80^{\circ} \mathrm{C}$. To prepare protein samples, the frozen tissue was homogenized in $900 \mu \mathrm{l}(\mathrm{Cx})$ or $200 \mu \mathrm{l}$ (PFC, Hipp, Thal) RIPA buffer with protease and phosphatase inhibitors (Roche), respectively. Homogenization was carried out with the Precellys homogenizer $(5500 \mathrm{rpm}, 2 \times 10 \mathrm{sec}$, Bertin Technologies), followed by lyses on ice for $20 \mathrm{~min}$. Lysed tissue was centrifuged for $10 \mathrm{~min}$ at $8000 \mathrm{rpm}$ at $4{ }^{\circ} \mathrm{C}$ in order to spin down the beads and unlysed material. The supernatant was transferred into a new Eppendorf cup and centrifuged for 30 min at maximum speed at $4{ }^{\circ} \mathrm{C}$. The supernatant containing the soluble proteins was transferred to a new Eppendorf cup. Supernatant and pellets were kept at $-80{ }^{\circ} \mathrm{C}$ until use. Protein concentration measurement was performed on dilutions (1:1 up to 1:20 in RIPA buffer) of the supernatant fraction.

\subsubsection{Sucrose buffer protein extraction}

To analyze the phosphorylation status of ErbB receptors and downstream effectors, and to improve the western blot quality for transmembrane proteins, sucrose bufferbased protein extraction was used. Micro-dissected frozen mouse brain material from one hemisphere (Cx, PFC, Hipp, Thal) was homogenized in $900 \mu \mathrm{l}$ (Cx) or $200 \mu \mathrm{l}$ (PFC, Hipp, Thal) sucrose homogenization buffer with protease and phosphatase inhibitors (Roche), respectively. Homogenization was carried out with the Precellys homogenizer (5500 rpm, 2x10 sec, Bertin Technologies). $130 \mu \mathrm{l}$ of the resulting homogenate were added to $70 \mu$ of $4 x$ LDS sample buffer (NuPAGE) working solution including DTT. The samples were immediately denatured at $70^{\circ} \mathrm{C}$ for $10 \mathrm{~min}$ 
and frozen and stored at $-80^{\circ} \mathrm{C}$. The leftovers of the homogenates were frozen and stored at $-80^{\circ} \mathrm{C}$. Protein concentration measurement was performed on dilutions $(1: 1$ up to 1:20 in sucrose homogenization buffer) of these leftovers.

\subsubsection{Synaptosomal preparation}

Many different protocols for the isolation of synaptosomes have been developed since its first description in 1958 (HEBB and WHITTAKER, 1958). Synaptosomes are resealed nerve terminals separated from axons and dendrites (Whittaker, 1993), they are very small (50 $\mathrm{nm}$ in diameter) and homogeneous in shape and size, and can be isolated by size fractionation techniques. The protocol used in this study was adapted from protocols described by Hell and Jahn (2006), Huttner et al. (1983) and Biesemann et al. (2014).

To isolated synaptosomes the tissue was prepared freshly. Mice were sacrificed by cervical dislocation followed by decapitation. The brain was immediately removed from the skull and the forebrain was dissected on ice. The cerebellum and olfactory bulb were removed and after separation of the two hemispheres, the midbrain was pinched out using a pair of bend tweezers and a dissection spatula. White matter was removed and the forebrains were transferred into ice-cold $0.32 \mathrm{M}$ sucrose homogenization buffer (synaptosomal extraction buffer) with protease and phosphatase inhibitors (Roche) and kept on ice. All used solutions were prepared with protease and phosphatase inhibitors (Roche) to ensure phospho-specificity for later analyses (Fig. 33).

The two forebrain hemispheres of each mouse were homogenized in $4 \mathrm{ml}$ of fresh ice-cold homogenization buffer with 12 gentle up and down strokes at 900 rpm using a loosely fitting $5 \mathrm{ml}$ glass-Teflon potter in the $4{ }^{\circ} \mathrm{C}$ cold room. After transfer of the homogenate to a centrifuge tube the potter was rinsed with additional $4 \mathrm{ml}$ of homogenization buffer in 9 up and down strokes. These $8 \mathrm{ml}$ of homogenate $(\mathrm{H})$ were centrifuged for $10 \mathrm{~min}$ at $1000 \mathrm{xg}$ at $4{ }^{\circ} \mathrm{C}$ in the SS-34 rotor (with adaptors) in the Sorvall centrifuge. This centrifugation step removed large cell fragments and nuclei from the solution. The supernatant (S1) was removed carefully from the pellet (P1). $\mathrm{P} 1$ was carefully resuspended in $4 \mathrm{ml}$ of homogenization buffer and centrifuged again for $10 \mathrm{~min}$ at $1000 \mathrm{xg}$ at $4{ }^{\circ} \mathrm{C}$ in the SS-34 rotor (with adaptors) in the Sorvall centrifuge. The supernatant (S1.2) was added to $S 1$ and centrifuged for $15 \mathrm{~min}$ at $12.500 \mathrm{xg}$ at $4{ }^{\circ} \mathrm{C}$ in the SS-34 rotor (with adaptors). The supernatant (S2) was removed completely and the synaptosome-enriched pellet (P2) was resuspended in $1 \mathrm{ml}$ of homogenization buffer. The P2 fraction was then carefully layered on top of a two-step sucrose density gradient consisting of $5 \mathrm{ml}$ of $1.2 \mathrm{M}$ and $5 \mathrm{ml}$ of $0.8 \mathrm{M}$ 
sucrose gradient solutions. After carefully balancing the tubes, the gradient was centrifuged for $2 \mathrm{~h}$ at $\sim 50.000 \mathrm{xg}(18.000 \mathrm{rpm})$ at $4{ }^{\circ} \mathrm{C}$ in a SW-41Ti swing-out bucket rotor in the Beckman ultracentrifuge with accel: 5 and deccel: 5 settings. The resulting gradient consisted of the three sucrose gradient steps $(0.32 \mathrm{M}, 0.8 \mathrm{M}$ and $1.2 \mathrm{M}$ sucrose solutions) containing low amounts of protein, two interfaces and a pellet. A myelin-containing fraction ' $A$ ' was enriched at the border of $0.32 \mathrm{M}$ to $0.8 \mathrm{M}$ sucrose. Synaptosomes were enriched in the interface ' $B$ ' of $1.2 \mathrm{M}$ and $0.8 \mathrm{M}$ sucrose and recovered by carefully removing the layers of the gradient step by step. The resulting fraction was referred to as synaptosome fraction or gradient-purified synaptosomes. $900 \mu \mathrm{l}$ of fraction B were then diluted with $900 \mu \mathrm{l}$ of $0.32 \mathrm{M}$ sucrose homogenization buffer and centrifuged for $20 \mathrm{~min}$ at $20.000 \mathrm{xg}(10.800 \mathrm{rpm})$ at $4{ }^{\circ} \mathrm{C}$ in the SW-41Ti swing-out bucket rotor in the Beckman ultracentrifuge, to wash and concentrate the synaptosomal fraction. The pellet (P3) was resuspended in $200 \mu \mathrm{l}$ of $0.32 \mathrm{M}$ sucrose homogenization buffer and referred to as final synaptosomes. Aliquots of all fractions were frozen and stored at $-80^{\circ} \mathrm{C}$. The pellet fractions $\mathrm{P} 1.2$ and the gradient pellet were resuspended in $800 \mu \mathrm{l}$ and $200 \mu \mathrm{l} 0.32 \mathrm{M}$ sucrose homogenization buffer, respectively, for protein concentration measurement and western blot analyses.

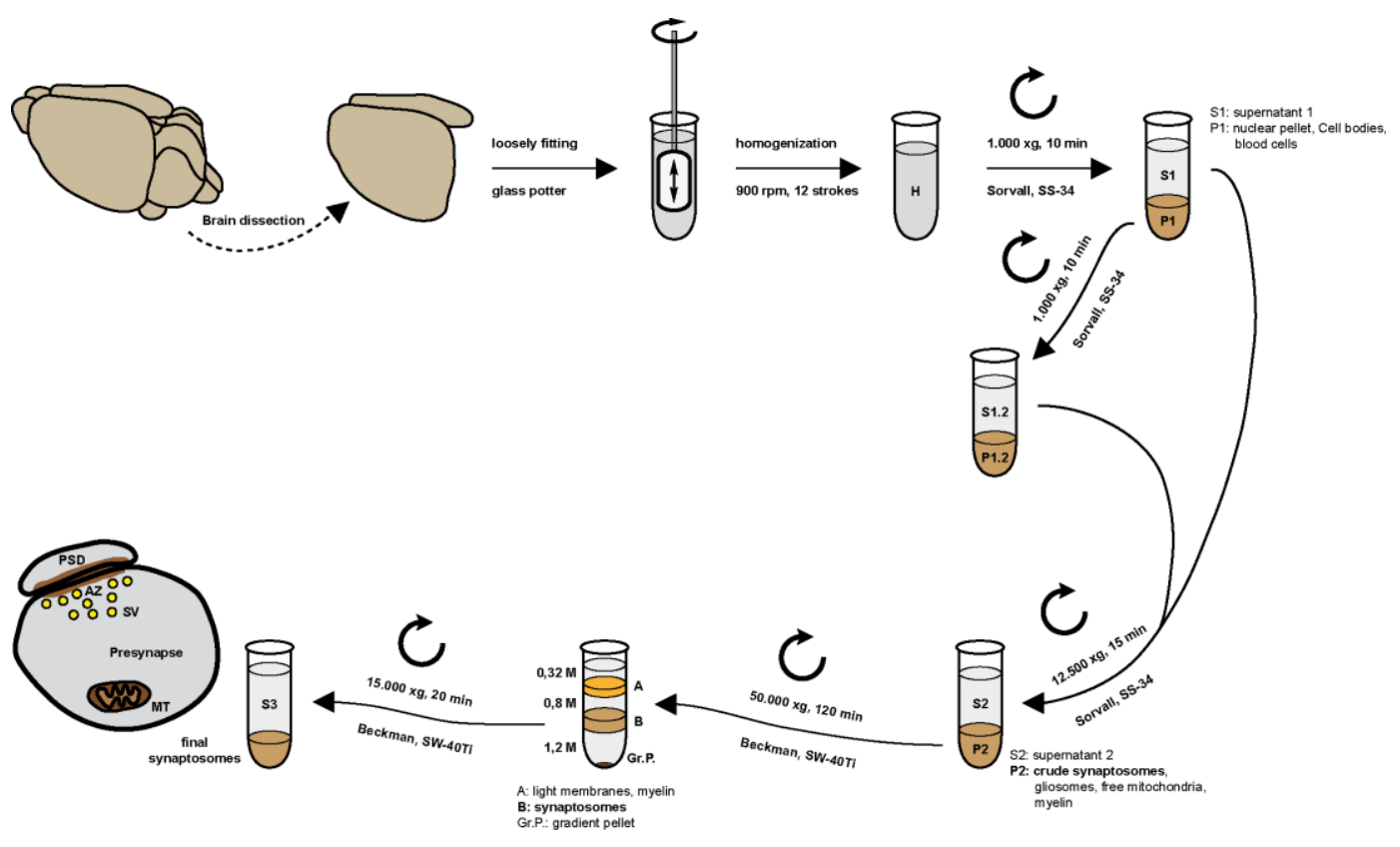

Fig. 33: Preparation of crude synaptosomal fractions 


\subsubsection{Protein concentration measurement with the Lowry assay}

To determine the protein concentration of samples the Bio-Rad DC Protein Assay kit was used. The working principal of this kit is based on the Lowry assay described in 1951 (LOWRY et al., 1951). The two step assay is based on the reaction between protein and an alkaline copper tartrate solution, followed by the reduction of Folin reagent that produces several reduced species of characteristic blue color with a maximum absorbance at $750 \mathrm{~nm}$. Hereby the color development is primarily due to oxidation of the amino acids tyrosine and tryptophan, and to a lesser extent cystine, cysteine, and histidine (LOWRY et al., 1951; Peterson, 1979). The assay was performed in a 96-well plate (flat bottom) according to the manufacturer's "micro plate" assay protocol and absorbance was measured at $650 \mathrm{~nm}$ with a microtitre plate reader (ThermoMax Molecular Devises). A standard curve, using dilutions of bovine serum albumine (BSA), was used to estimate protein concentrations. Protein samples were pre-diluted before measurement and concentrations were calculated with regard to the dilution factor.

\subsubsection{Preparation of protein samples for SDS electrophoresis}

\subsubsection{RIPA buffer protein samples}

RIPA buffer extracted protein samples were set up with final concentrations ranging between 2.5 to $5 \mu \mathrm{g} / \mu \mathrm{l}$ in $5 x$ SDS sample buffer with $1 \% \beta$-mercaptoethanol. Volumes of the samples were equalized with RIPA buffer. Samples were mixed carefully by pipetting up and down and afterwards denatured at $95^{\circ} \mathrm{C}$ for $10 \mathrm{~min}$. For storage samples were kept at $-80^{\circ} \mathrm{C}$.

\subsubsection{Sucrose buffer protein samples}

Since sucrose buffer extracted samples were set up and denatured in $4 x$ LDS sample buffer directly after homogenization, the protein concentration measurement was carried out on the leftover of the homogenate. Using the measured concentrations, denatured samples were then diluted to equal concentrations (ranging between 1 and $3 \mu \mathrm{g} / \mu$ l protein) using a similar mixture of $4 x$ LDS samples buffer with DTT and sucrose homogenization buffer as used in the denatured sample. Diluted and undiluted samples were stored at $-80^{\circ} \mathrm{C}$ until use.

\subsubsection{Synaptosomes}

Samples of the different fractions of the synaptosomal preparation were set up for western blot analyses in two different ways. In one approach samples were set up 
using same volumes $(20 \mu \mathrm{l})$ of the fractions in $4 x$ LDS sample buffer with DTT. In the second approach samples were set up in equal concentrations ( $1 \mu \mathrm{g} / \mu \mathrm{l}$ protein) in $4 \mathrm{x}$ LDS sample buffer with DTT. Volumes of samples were equalized with $0.32 \mathrm{M}$ sucrose homogenization buffer. In both cases samples were denatured at $70{ }^{\circ} \mathrm{C}$ for $10 \mathrm{~min}$.

\subsubsection{Discontinous SDS polyacrylamide gel electrophoresis}

To separate proteins by size from a sample, discontinuous SDS polyacrylamide gel electrophoresis (SDS-PAGE) was performed (Laemmli, 1970). SDS polyacrylamide gels were casted between Bio-Rad glass plates and $0.75 \mathrm{~mm}$ or $1.75 \mathrm{~mm}$ spacer plates that were assembled according to the company's instructions. The separation gel acrylamide solution of the desired percentage was poured between the glass plates, separated from air by a layer of isopropyl alcohol and left to polymerize for 30 min at RT. After rinsing the isopropyl alcohol with $\mathrm{ddH}_{2} \mathrm{O}$, the stacking gel solution was added onto the separation gel. A Teflon comb (10 or 15 teeth) was immediately inserted into the stacking gel solution and the gel was left to polymerize for $30 \mathrm{~min}$. Polymerized gels were kept up to one week at $4{ }^{\circ} \mathrm{C}$. The chamber for electrophoresis (Bio-Rad mini protean 3) was assembled according to the manufacturer's manual and the inner gasket was filled with the electrophoresis buffer to check for leakiness of the chamber. The combs were carefully removed and the wells were washed with electrophoresis buffer by pipetting up and down. 3-30 $\mu \mathrm{g}$ of denatured protein samples and $10 \mu \mathrm{l}$ of prestained protein marker (Fermentas) were loaded onto the gel. The chamber was filled with electrophoresis buffer and connected to a power supply (Bio-Rad). The gel was run under a constant voltage of $100 \mathrm{mV}$ until samples aligned at the border of stacking and separation gel. The voltage was then increased to $150 \mathrm{mV}$ and the gel was run until the tracking dye reached the end of the gel. The gel was carefully removed from the glass plates and proceeded with Coomassie staining or western blot analysis.

\subsubsection{Coomassie staining}

To verify the protein transfer, SDS-PAGE gels were stained with Coomassie solution. Briefly, the gel was covered with Coomassie solution and coocked in a microwave for 30 sec. Afterwards gels were washed with $\mathrm{ddH}_{2} \mathrm{O}$ at RT under constant gentle shaking until the protein bands were clearly visible. 


\subsubsection{Western Blotting}

\subsubsection{Electrophoretic transfer}

For immunological detection of proteins resolved by SDS-PAGE, proteins were transferred onto PVDF membranes by electrophoresis, as originally described by Towbin and colleagues (Towbin et al., 1979). By applying an electric current to the blotting module, the negatively charged proteins migrate from the cathode to the anode and are retained on a PVDF membrane by electrostatic and hydrophobic interactions. PVDF membranes (Roche, pore size $0.2 \mu \mathrm{m}$ ) were activated for $1 \mathrm{~min}$ in $100 \%$ methanol, washed twice in $\mathrm{dd}_{2} \mathrm{O}$ and then placed into transfer buffer. Blotting pads and Whatman papers were pre-soaked in transfer buffer and the blotting sandwich was assembled in the following way:
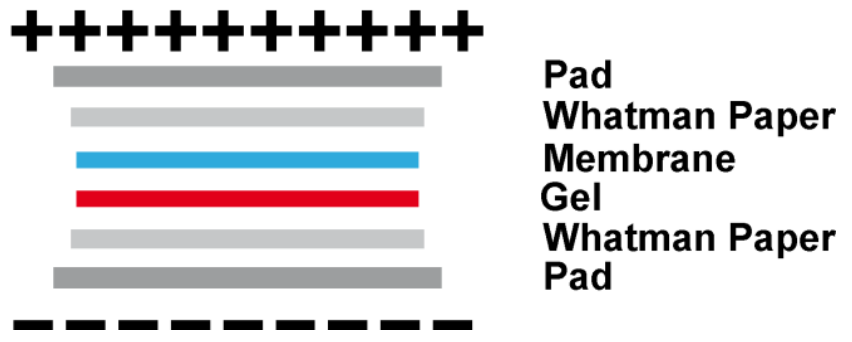

Fig. 34: Bio-Rad Mini Trans-Blot western blot sandwich

The protein transfer was performed using Bio-Rad Mini Trans-Blot ${ }^{\circledR}$ cells under a constant voltage of $38 \mathrm{~V}$ and a maximum current of $275 \mathrm{~mA}$, for $90-180 \mathrm{~min}$ (depending on the size of the protein and the thickness of the gel) at $4{ }^{\circ} \mathrm{C}$.

\subsubsection{Immunological detection of proteins on PVDF membranes}

After electrophoretic transfer of proteins to PVDF membranes, membranes were first blocked for $60 \mathrm{~min}$ at RT in blocking buffer and then placed into $50 \mathrm{ml}$ falcons with 3 $\mathrm{ml}$ of the primary antibody diluted in blocking buffer. Incubation was carried out $\mathrm{ON}$ at $4{ }^{\circ} \mathrm{C}$ with constant rotation. On the next day membranes were washed four times for 10 min TBST and incubated with the HRP-conjugated secondary antibody diluted in blocking buffer for $1 \mathrm{hr}$ at RT. The membranes were again washed four times with TBST for $10 \mathrm{~min}$ at RT, followed by $1 \mathrm{~min}$ incubation with a $1: 1$ dilution of the two Enhanced Chemiluminescence Detection (ECL) solutions. After drying the membranes on tissue, they were placed into a transparent plastic foil and placed into the Intas ChemoCam Imager. The membranes were scanned for $20 \mathrm{~min}$, the protein standard fotographed and the images saved digitally for later analyses and quantifications.

To reprobe membranes with different antibodies, membranes were incubated in 
stripping buffer for $1 \mathrm{hr}$ at $60{ }^{\circ} \mathrm{C}$ or in $0.5 \mathrm{M} \mathrm{NaOH}$ for 15 min at RT with vigorous shaking. After washing in TBST and blocking, membranes were incubated in the next antibody.

\subsubsection{Densitometric quantification of band intensity}

For densitometric analyses of western blot bands, raw images acquired with the Intas ChemoCam Imager were opened with FIJI, converted to 8-Bit and inverted (to have black bands on white background). The densities of bands of interest were measured using FIJl's gel analyzing function. Results were normalized to loading controls (e.g. $\beta$-actin or $\alpha$-tubulin) and normalized values ( \pm SEM) were analyzed for statistical significance using the GraphPad Prism software package.

\subsection{Histology and immunohistochemistry}

\subsubsection{Analysis of fluorescent organs}

The transgene cassette of Stop-Nrg1 mice contains a loxP sites-flanked eGFP Stopcassette. This element allows for Cre-dependent stage-specific activation of the HACRD-NRG1 overexpression, but can also be used to genotype mice and to visualize transgenic cells. To check which organs of the mice expressed the transgene cassette, different organs (brain, heart, liver, muscles, tailtips, spinal cord and sciatic nerves) were examined for eGFP fluorescence. Mice were sacrificed by cervical dislocation and decapitation, organs were dissected from the mice and after short rinsing in $1 \mathrm{x}$ PBS, they were examined with the Leica MZ16F fluorescent stereomicroscope. Images of brightfield and fluorescent light were taken and analyzed by FIJI software.

\subsubsection{Perfusion fixation of mouse tissue for histology}

To perform histology on mouse brain tissue, mice were perfusion fixed to better preserve the tissue for later processing. Mice were deeply anesthetize by IP injection of Avertin with a dose of $0.2 \mathrm{ml}$ per $10 \mathrm{~g}$ of body weight. Once the mouse was anesthetized, it was fixed onto a preparation platform with drainage (ventral side up). Two pieces of tail were cut for later re-genotyping and the abdomen and the diaphragm were opened, and the rib cage was cut laterally on both sides to the top and above the sternum. It was then fully removed to expose the heart. A 27 gauge needle butterfly connected to a peristaltic pump was carefully inserted into the left ventricle, making sure not to pierce the septum, and the right atrium was opened by 
a small cut to ensure the perfusion flow. The peristaltic pump was started and the blood was flushed out of the circulatory system with $20 \mathrm{ml}$ of pre-warmed HBSS at a flow rate of $1 \mathrm{ml} / \mathrm{min}$. Afterwards the mouse was perfused with $30-40 \mathrm{ml}$ of ice-cold $4 \%$ PFA fixative at a flow rate of $1 \mathrm{ml} / \mathrm{min}$. After perfusion, fixed brains were dissected and placed into cold fixative for post-fixation. Post-fixation was carried out $\mathrm{ON}$ at $4{ }^{\circ} \mathrm{C}$. Tissue for vibratome sectioning was sectioned directly after $\mathrm{ON}$ post fixation. For cryosectioning of brain tissue, post-fixed tissue was cryo protected in sucrose solutions (see section 6.5.4). Tissue for paraffin embedding was changed to $1 \%$ PFA, after post-fixation, for prolonged storage at $4{ }^{\circ} \mathrm{C}$, or immediately proceeded for embedding (see section 6.5.5).

\subsubsection{Vibratome sectioning of tissue}

For vibratome sectioning, tissue was, after post-fixation, washed in 1x PBS and immediately sectioned at the vibratome. Therefore the brains were sliced coronally into 3 three pieces of $4 \mathrm{~mm}$ thickness, a PFC, a hippocampal and a cerebellar section. These pieces were glued onto the specimen holder of the vibratome using instant glue. The specimen holder was fixed onto the stage of the vibratome that was filled with ice cold 1x PBS. Coronal sections of $50 \mu \mathrm{m}$ thickness were sectioned and collected in 24-well plates in $1 x$ PBS. The sections were kept at $4{ }^{\circ} \mathrm{C}$ and were immediately proceeded with immunohistological stainings.

\subsubsection{Cryo protection, embedding and cryosectioning of tissue}

For cryo sectioning, mouse brains were cryo protected by incubations in a row of sucrose solutions with accending sucrose concentrations. First tissue was shortly rinsed in 1x PBS and then submersed in a $10 \%(w / v)$, a $20 \%(w / v)$ and finally a 30 $\%(w / v)$ sucrose solution at $4{ }^{\circ} \mathrm{C}$, until the tissue had sunk to the bottom of the tube in each solution. Brains were sectioned into three $4 \mathrm{~mm}$ thick coronal pieces (a PFC, hippocampal and cerebellar piece) using a brain slicer, and frozen on aluminum foil on dry ice, then wrapped in aluminum foil and stored until sectioning at $-80{ }^{\circ} \mathrm{C}$. Embryonic brains of Stop-Nrg ${ }^{*} N E X$-Cre mice, that were used to determine the early embryonic onset of HA-Nrg1 overexpression, were, due to the tissue size, embedded in Shandon cryomatrix in aluminum foil molds. A layer of Shandon cryomatrix was filled into the labeled molds at RT and the cryo protected tissue was placed into the molds with the cutting surface facing to the bottom of the mold. Molds were carefully filled with Shandon cryomatrix and placed onto dry ice to freeze. For sectioning tissue was transferred from $-80{ }^{\circ} \mathrm{C}$ to the cryostat $\left(-21^{\circ} \mathrm{C}\right)$ half an hour prior to 
sectioning. Shandon cryomatrix-embedded embryonic tissue blocks were trimmed to size using razor blades. Afterwards brain pieces or cryomatrix blocks were glued onto a specimen holder with Shandon cryomatrix embedding medium and fixed into the cryostat. $14 \mu \mathrm{m}$ to $20 \mu \mathrm{m}$ thick sections were cut using a knife-angle of $4^{\circ}$, a chamber temperature of $-21^{\circ} \mathrm{C}$ and an object temperature of $-19^{\circ} \mathrm{C}$. Sections were either collected on positively charged ultrafrost microscope slides, dryed ON at RT in an exsiccator and used for "on slide" immunohistochemistry, or collected in 1x PBS in multi-well plates and processed for stainings on free-floating cryo sections.

\subsubsection{Paraplast impregnation, embedding and sectioning of tissue}

For paraplast embedding, tissue was, after ON post-fixation, shortly rinsed in 1x PBS and sliced into three $4 \mathrm{~mm}$ thick pieces (a PFC, hippocampal and cerebellar section). The three pieces were given into tissue cassettes and using the paraplast embedding station (see embedding scheme below), dehydrated and infiltrated with paraplast, and then embedded into paraplast blocks, using the paraplast embedding centre and metall molds. After cooling down harden the fresh paraplast, blocks were removed from the molds and stored at $\mathrm{RT}$ until sectioning.

Paraplast blocks were sectioned using a sliding microtome and the $5 \mu \mathrm{m}$ thick sections were collected on ice-cold destilled water. After transfer to a $42{ }^{\circ} \mathrm{C}$ waterbath and straigthening, sections were mounted onto charged microscope slides. For parallel processing, sections of multiple genotypes were mounted per slide (for instance Cre and Stop-Nrg1 parental controls next to a Stop-Nrg $1{ }^{\star} \mathrm{Cre}$ double transgenic mouse). Sections were dried at RT between each animal, and after the last section, slides were dried $\mathrm{ON}$ at $37{ }^{\circ} \mathrm{C}$ and then stored at RT or proceeded with fluorescent and chromogenic immunohistological stainings.

Embedding scheme of the paraplast embedding centre:

Solution

EtOH $50 \%$

$\mathrm{EtOH} 70 \%$

EtOH $70 \%$

EtOH $96 \%$

EtOH $96 \%$

EtOH $100 \%$

EtOH $100 \%$

Isopropanol

Xylol

Xylol

Paraplast

Paraplast

\section{Incubation time}

1 hour

2 hours

2 hours

1 hour

1 hour

1 hour

1 hour

1 hour

2 hours

2 hours

2 hours

2 hours 


\subsubsection{Histological staining procedures}

\subsubsection{Haematoxylin-Eosin $(\mathrm{H}+\mathrm{E})$ staining}

Haematoxylin-Eosin $(\mathrm{H}+\mathrm{E})$ staining is widely used as a histological staining method that gives information of the overall tissue structure. Haematoxylin stains basophilic nuclei in dark purple and Eosin stains eosinophilic cytoplasm pink.

For the $\mathrm{H}+\mathrm{E}$ staining, paraffin sections were deparaffinised by two $10 \mathrm{~min}$ incubations in Xylol and one for $10 \mathrm{~min}$ in Xylol/lsopropanol (1:1), followed by rehydratation in descending ethanol dilutions (100\%, $90 \%, 70 \%$, and $50 \%$ ) for 5 min each. After washing for $5 \mathrm{~min}$ in $\mathrm{ddH}_{2} \mathrm{O}$, sections were stained with $0.1 \%$ Haematoxylin for $5 \mathrm{~min}$ and washed with $\mathrm{ddH}_{2} \mathrm{O}$. The dark purple color was differentiated by a short wash in $\mathrm{HCl}$-Alcohol solution. To stop the differentiation process and properly develop the color, sections were incubated for $5 \mathrm{~min}$ in Scott's solution. After a short rinse in $\mathrm{dd}_{2} \mathrm{O}$, sections were counterstained in $0.1 \%$ Eosin for 3-5 min. Excess dye was washed off with $\mathrm{dd}_{2} \mathrm{O}$. After dehydration in an ascending row of ethanol dilution ( $50 \%, 70 \%, 90 \%$, and $100 \%$ ) by short incubation steps (10-30 sec, depending on the stability of the Eosin stain), sections were incubated once for $10 \mathrm{~min}$ in Xylol/Isopropanol (1:1) and twice for $10 \mathrm{~min}$ in Xylol, and finally mounted with the Xylol based mounting medium Eukitt and dried at RT.

\subsubsection{DAB immunostaining of paraffin sections}

$D A B$ immunostaining is based on the enzymatic reaction of horseradish peroxidase (HRP) attached to a secondary antibody with DAB substrate, which forms a stable brown precipitate that can be visualized by light microscopy (Harlow and Lane, 1988). $5 \mu \mathrm{m}$ thick paraffin sections were incubated at $60{ }^{\circ} \mathrm{C}$ for $10 \mathrm{~min}$ and deparaffinized and dehydrated as for the $\mathrm{H}+\mathrm{E}$ staining. Afterwards, sections were incubated for $5 \mathrm{~min}$ in $\mathrm{ddH}_{2} \mathrm{O}$ followed by $5 \mathrm{~min}$ incubation in citrate buffer. Meanwhile citrate buffer was heated up in a microwave. Sections were cooked for 10 min in boiling citrate buffer at $650 \mathrm{~W}$ in the microwave for antigen retrieval. Eventual evaporation was replenished with $\mathrm{dd}_{2} \mathrm{O}$, to maintain the buffer molarity. After a cool down at RT for 20 min or longer, sections were washed for 5 min in Tris-buffer with 2 $\%$ milk powder and then mounted with plastic cover-plates for the following steps of the protocol. To test if cover-plates were mounted properly and the flowrate was optimal, sections were washed with Tris-buffer with $2 \%$ milk powder. Then endogenous peroxidases were inactivated by 5 min incubation with $100 \mu \mathrm{l}$ of $3 \%$ hydrogen peroxide followed by blocking with $100 \mu \mathrm{l}$ of blocking buffer for $20 \mathrm{~min}$ at RT. Then $100 \mu$ of the primary antibody in PBS/BSA were applied to the slides and 
incubated $\mathrm{ON}$ at $4{ }^{\circ} \mathrm{C}$. On the next day, sections were washed with Tris-buffer with 2 $\%$ milk-powder, followed by incubation with $100 \mu \mathrm{l}$ of the biotinylated secondary antibody (Dako LSAB2, yellow bottle solution A) for 10 min at RT. After washing in Tris-buffer with $2 \%$ milk-powder, $100 \mu \mathrm{l}$ of Steptavidine conjugated to Horseradish Peroxidase (Dako LSAB2, red bottle solution B) were added to the sections and incubated for another $10 \mathrm{~min}$ at RT. After washing with Tris-buffer without milkpowder for $10 \mathrm{~min}$ at RT, plastic cover-plates were removed and slides were placed into a box for staining with $100 \mu \mathrm{l}$ of DAB substrate solution $(1 \mathrm{ml}$ Dako Substrate buffer with two drops of DAB) for $10 \mathrm{~min}$, followed by washing twice in $\mathrm{dd}_{2} \mathrm{O}$ for 5 min. Depending on the purpose of the staining, sections were either counterstained with Haematoxylin for $30 \mathrm{sec}$ and then washed once in $\mathrm{dd}_{2} \mathrm{O}$ for $5 \mathrm{~min}$, or sections were, without counterstaining, directly proceeded with dehydration in the ascending alcohol row and mounting in Eukitt, as for the $\mathrm{H}+\mathrm{E}$ staining.

\subsubsection{DAB immunostaining of paraffin sections using the Ventana staining machine}

For quantification of neuronal numbers, paraffin sections were stained chromogenically using the Ventana Discovery XT Staining Module for standardized, automated stainings, that provide higher comparability between different sections. In addition, the staining procedure in an oil-based medium at $37^{\circ} \mathrm{C}$ also speed up the staining process. First, microscope slides were labeled with a barcode sticker, encoding staining informations. Staining was carried out using the Research $\mathrm{IHC}$ DAB Map XT protocol with the Research IHC DAB Map XT kit according to the manufacturer's informations. Primary antibodies and biotin-conjugated bridging antibodies were titrated by hand. Primary antibodies were incubated for $60 \mathrm{~min}$, secondary biotin-conjugated antibodies for $20 \mathrm{~min}$ at $37^{\circ} \mathrm{C}$. Afterwards, sections were collected from the machine and rinsed with soapy water in a glas cuvette, to remove the residual oil from the slides. After dehydration by short incubations in an ascending row of alcohol solutions, followed by one incubation for $10 \mathrm{~min}$ in Xylol/Isopropanol (1:1) and twice for $10 \mathrm{~min}$ in Xylol, sections were mounted with Eukitt mounting medium and dried at RT.

\subsubsection{Fluorescent immunostaining of paraffin sections}

The protocol for fluorescent immunostaining of paraffin sections is similar to the DAB staining protocol until the steps after antigen retrieval. After cooling down, sections were washed $3 x$ for 5 min in Tris-buffer with $2 \%$ milk-powder, and placed into a 
humidified chamber. $150 \mu \mathrm{l}$ of blocking buffer were pipetted onto each slide and slides were covered with a piece of parafilm to ensure equal distribution of the blocking solution, allowing application of low (as low as $100 \mu \mathrm{l}$ ) volumes to the slides. Blocking was carried out for $20 \mathrm{~min}$ at RT. After decanting the blocking buffer, $150 \mu \mathrm{l}$ of primary antibody were applied, slides were again covered with parafilm and incubated $\mathrm{ON}$ at $4{ }^{\circ} \mathrm{C}$ in the humidified chamber. On the next day, slides were washed $3 x$ for 5 min in Tris-buffer with $2 \%$ milk-powder, followed by application of $150 \mu$ dilution of fluorescent secondary antibody (incl. DAPI) in PBS/BSA and covering with parafilm. Incubation was carried out for 1 hour at RT in the humidified chamber, followed by $3 x$ washing in Tris-buffer without milk-powder for $5 \mathrm{~min}$, mounting with AquaPolymount, and storage at $4{ }^{\circ} \mathrm{C}$.

\subsubsection{Fluorescent staining of vibratome sections}

Free floating vibratome sections were stained in a 24-well plate, starting with a 30 min permeabilization in $200 \mu \mathrm{l}$ of $0.4 \%$ Triton X-100 in 1x PBS at RT. Afterwards, solution was carefully removed with a pipette and $200 \mu \mathrm{l}$ of blocking solution were applied (4\% Horse-Serum, $0.1 \%$ Triton X-100 in 1x PBS) for 30 min at RT. Primary antibodies (150 $\mu$ l per slide) were diluted in $1 \%$ Horse-Serum, $0.1 \%$ Triton X-100 in 1x PBS and incubated $\mathrm{ON}$ at $4{ }^{\circ} \mathrm{C}$. On the next day sections were washed $3 x$ for 5 min in 1x PBS $(500 \mu \mathrm{l})$, before application of $150 \mu \mathrm{l}$ of the fluorescent secondary antibodies and Dapi (1 mg/ml), diluted in $1.5 \%$ Horse-Serum in 1x PBS, and incubation for 2 hours at RT. From this step on slides had to be protected from light. After three more washing steps with $1 x$ PBS, sections were carefully transferred with a soft brush to a deep petridish filled with tap water and pulled onto charged microscope slides. After a short first drying, sections were mounted with AquaPolymount and stored at $4{ }^{\circ} \mathrm{C}$.

\subsubsection{Fluorescent staining of cryo sections}

Free floating cryo sections were stained, using the similar protocol as for free floating vibratome sections. Staining of slide-mounted cryo sections, was carried out on lying slides in a humidified box. Shandon cryomatrix was washed off by 10 min incubation in $1 \mathrm{x}$ PBS at RT in a cuvette, prior to permeabilization and blocking. Slides were placed into a humidified chamber and $200 \mu$ of $4 \%$ Horse-Serum, $0.1 \%$ Triton X100 in 1x PBS were applied onto the slides for combined permeabilization and blocking for $30 \mathrm{~min}$ at RT. Afterwards $150 \mu \mathrm{l}$ of the primary antibodies (diluted in $1 \%$ Horse-Serum, $0.1 \%$ Triton X-100 in 1x PBS) were applied and, after covering with 
parafilm, incubation was carried out $\mathrm{ON}$ at $4{ }^{\circ} \mathrm{C}$. On the next day the staining was proceeded with three washing steps of $10 \mathrm{~min}$ in $1 \times$ PBS at RT in a cuvette, followed by application of $150 \mu \mathrm{l}$ of the secondary antibodies and Dapi diluted in $1.5 \%$ HorseSerum in 1x PBS, covering with parafilm, and incubation for two hours at RT in the humidified chamber. Afterwards, slides were washed $3 x$ in $1 x$ PBS in a cuvette, protected from light, then mounted in AquaPolymount and stored at $4{ }^{\circ} \mathrm{C}$.

\subsubsection{Detection of $\beta$-galactosidase in tissue sections (X-Gal staining)}

The X-Gal staining method is based on the bacterial enzyme $\beta$-galactosidase's ( $\beta$ gal) catalytic cleavage $\beta$-galactosides into monosaccharides. The enzyme has a broad substrate specificity and can thus also be used to cleave organic compounds such as 5-bromo4-chloro-3-indolyl- $\beta$-D-galactoside (X-Gal), resulting in a colorful indigo-colored precipitate (5-bromo-4-chloro-3-hydroxyindole) under oxidizing buffer conditions. Placed under the appropriate regulatory elements, the enzyme can furthermore be expressed in mammalian cells and is usually well tolerated. In transgenic mice this property of the enzyme allows the use of $\beta$-gal as a reporter to detect transgene or promoter activity of endogenous genes in 'knock-in' approaches (Goring et al., 1987; Sanes, 1994).

To visualize $\beta$-gal activity in transgenic reporter mice, $50 \mu \mathrm{m}$ vibratome brain sections were incubated in ' $\beta$-gal staining buffer' for 10 min to 24 hours at $37^{\circ} \mathrm{C}$ in the dark. Sections were washed $3 x$ for 10 min in $1 x$ PBS at RT, to stop the X-Gal reaction. Sections were mounted onto charged microscope slides, shortly dried, then mounted with AquaPolymount and stored at $4{ }^{\circ} \mathrm{C}$.

\subsubsection{Imaging and image analysis}

\subsubsection{Cell counting of chromogenic stainings}

To quantify neuron numbers in the cortex and hippocampus of transgenic mice, $5 \mu \mathrm{m}$ thick coronal paraffin sections (bregma -1.7) were stained chromogenically (see section 6.5.6.2-3) for neuronal markers and then imaged as tiles overviews at $10 \mathrm{x}$ magnification using a Zeiss Imager.Z1 microscope. Cortical and hippocampal cell numbers were blindly counted using the cell counter plugin for FIJI software (http://fiji.sc/Cell_Counter). Counted cell numbers were expressed as cell densities (cells per area counted $\left(\mathrm{mm}^{2}\right)$ ). Cell numbers of both hemispheres were averaged and the data was analyzed using Excel and GraphPad Prism software. 


\subsubsection{Quantification of $\mathrm{GFAP}^{+}$and $\mathrm{IBA1}^{+}$areas in chromogenic stainings}

To determine the $\mathrm{GFAP}^{+}$or $\mid \mathrm{BA} 1^{+}$area in chromogenically stained coronal brain sections of transgenic mice, a semi-automatic analysis, using a plug-in for the FIJI software (available online at http://www1.em.mpg.de/gfap), was conducted. Briefly, 10x overview pictures, acquired with the Zeiss Imager.Z1 microscope, were blindly loaded into the software. Regions of interest (cortex, hippocampal grey matter, fimbria and the corpus callosum) were marked in both hemispheres and the software analyzed the DAB-positive area over the total area quantified. Area fractions of both hemispheres were averaged and the data was analyzed using Excel and GraphPad Prism software.

\subsubsection{Two-Photon laser scanning microscopy}

For 2P-LSM, transgenic mice of 6-8 weeks of age were anesthetized using a gas mixture of $\mathrm{O}_{2}: \mathrm{N}_{2} \mathrm{O}(1: 1)$ loaded with $5 \%$ isoflurane in a closed box (flow rate: 1000 $\mathrm{ml} / \mathrm{min}$ ). After initial sedation, anesthesia was maintained by a mask, on a heated plate and reduced flow rate $\left(\mathrm{N}_{2} \mathrm{O}: 100-200 \mathrm{ml} / \mathrm{min}\right.$; $\mathrm{O}_{2}: 200-300 \mathrm{ml} / \mathrm{min} ; 1.5-2 \%$ isoflurane). The respiration rate was kept below 2 per second by adjusting the isoflurane dosage and the body temperature was kept constant $\left(36-38{ }^{\circ} \mathrm{C}\right)$ throughout the experiment. The skull was attached to a custom-made ring using cement to reduce respiratory-induced movements. A cranial window close to the sagittal suture through the parietal bone was opened using a small dentist drill. The exposed cortex was covered by a glass coverslip.

With a custom-made 2P-LSM equipped with an fs-pulsed titanium-sapphire laser (Chameleon Ultra II, Coherent, Glasgow, UK) and a long-distance 203/1.0 NA water immersion objective (Zeiss, Jena, Germany), in vivo imaging was performed. For excitation, the laser was set at $925 \pm 5 \mathrm{~nm}$. The fluorescence signal of PV-GFP ${ }^{+}$ interneurons was collected by a photomultiplier tube (Hamamatsu, Japan) through a $510 \pm 42 \mathrm{~nm}$ band-pass filter (Semrock). Uniformly spaced $(0.8 \mu \mathrm{m})$ planes of $500 \mathrm{x}$ $500 \mu \mathrm{m}^{2}$ regions of the cerebral cortex were recorded and processed to obtain $z$ stacks of images (512 x 512 or $1024 \times 1024$ pixels in size). Image processing and 3D cell counting was performed using ImageJ (NIH; http://rsbweb.nih.gov/ij). For 3D visualization of image stacks as volumes, Java-based ImageJ 3D viewer plugin developed by Benjamin Schmid (Biozentrum) was used. 


\subsubsection{Data analysis and statistics}

Data organization, processing and analysis was performed using Microsoft Excel for Mac 2008 software. Statistical analyses were performed using GraphPad Prism software. Quantified values were grouped by animals and genotypes and averages with standard deviations (SD) and SEM (Standard error of the mean) were calculated. Statistical significance was analyzed with GraphPad Prism software using Mann-Whitney U-test and One-way ANOVA with Bonferroni post-test (for multiple group comparisons).

\subsection{Behavioral experiments}

Mice were brought to the behavioral unit at least one week before the experiments, to allow them to adapt to the new environment. Mice were kept group housed with their littermates, with feed and water ad libitum, air ventilation and under a constant 12 hour light-dark-cycle. Cage maintenance was done before and after the experimental phase, to reduce stress and handling during this phase. Animals were weighted before and after the experimental phase. Behavioral experiments were carried out during the light phase, with mice labeled only with default numbers to ensure blinding of the experimentator (blind to genotype). Animals were decoded and sorted into their respective groups only at the end of the study. Data was analyzed with Microsoft Excel for Mac 2008 and GraphPad Prism software. The majority of experiments performed in this study were performed according to standard protocols (Crawley, 2007). Unless stated otherwise, behavioral test apparatuses of TSE Systems (Bad Homburg) were used. Test chambers and apparatuses were cleaned with $70 \%$ Ethanol p.A. prior to the experiment and between each trial, to minimize olfactory cues and ensure equal conditions for every mouse.

\subsubsection{Open field test}

The open field test is a widely used test to monitor spontanious locomotoric activity and anxiety in rodents (Hall, 1934; Broadhurst, 1961). Animals were allowed to explore an open field arena (size: $45 \times 45 \times 55 \mathrm{~cm}$ ), with transparent walls and grey PVC floor (Fig. 35), for $10 \mathrm{~min}$ with automatic tracking by an $x-y-z$ infrared observation system. Data was recorded using ActiMot software of TSE Systems. The following parameters were recorded: distance travelled in $\mathrm{m}$, time spend in the center ( $70 \%$ of the area) or the periphery (30\% of the area) of the arena, travel speed, time active, corner visits and rearings. 


\subsubsection{Hole board test}

To analyze the curiosity of mice, the hole board test (Bradley et al., 1968) was used. Holes in the floor of the experimental chambre triggered exploratory behavior, e.g. nos poking. The test was performed in the same apparatus as the open field test, only a second floor with 16 holes (diameter: $2 \mathrm{~cm}$ ) was inserted into the arena (Fig. $35)$. In this case, the $x-y-z$ detectors of the system monitored nose pokes. Again the mice had $10 \mathrm{~min}$ to explore the arena. The system automatically tracked the distance travelled, number of nose pokes, and exploration time.
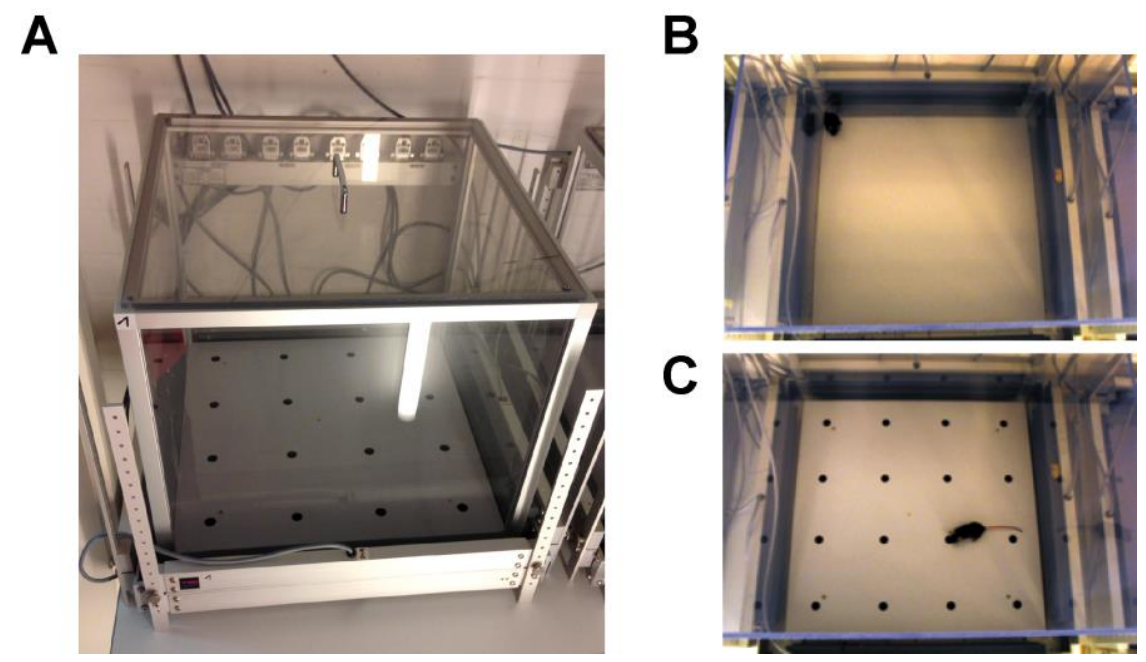

Fig. 35: TSE Systems open field and hole board system. (A) Arena with infrared detector system. (B) Arena in open field test mode and (C) with hole board inserts. (Pictures in B and C were kindly provided by M. Brzózka).

\subsubsection{Hot plate test}

The hot plate test (O'Callaghan and Holtzman, 1975) was used to monitor the pain sensitivity of mice. Similar pain sensitivity in transgenic and control mice is crucial for certain learning tests, such as the fear conditioning test.

Pain sensitivity was analyzed by placing mice onto a $55^{\circ} \mathrm{C}$ hot plate surrounded by a plexiglass cylinder (Fig. 36) and measuring the time until they started licking their hindpaws (,latency to lick the hindpaws (s)'). As soon as mice started to show this reaction, they were removed from the hot plate and placed onto a cool metal surface to cool down and were then transferred back into their homecages. Animals that managed to jump out of the cylinder were excluded from the analysis. 


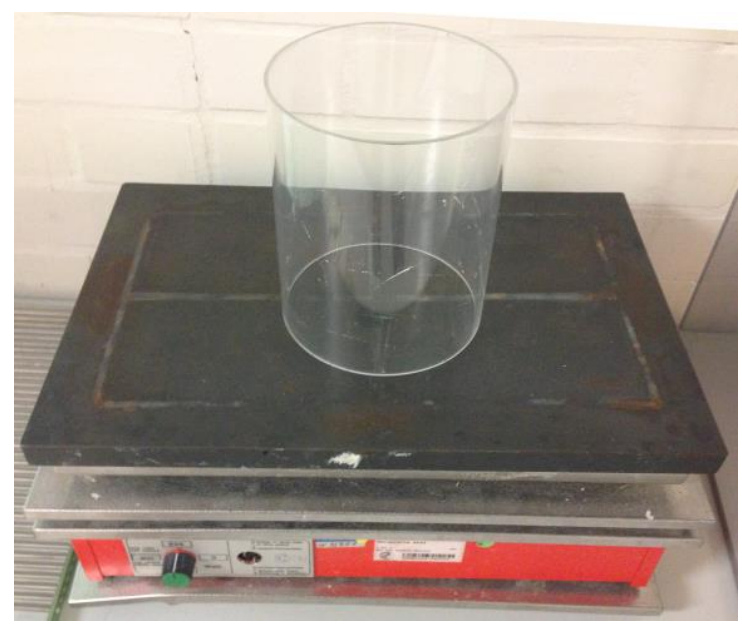

Fig. 36: Hot plate test setup. $55^{\circ} \mathrm{C}$ hot plate and a plexiglass cylinder to prevent mice from escaping.

\subsubsection{Light-Dark preference test}

The conflict between the drive to explore a novel environment and the aversion toward a light room was used for the light-dark preference test (Crawley and Goodwin, 1980). The in-house build experimental chamber consisted of two parts, a light compartment (LC) that was surrounded by transparent walls and a dark compartment (DC), with dark walls (Fig. 37). Both compartments were connected by a small open door. The test started by placing a mouse into the light compartment, facing towards the outside wall. The time until the mouse first entered the dark compartment was stopped (,latency to enter the dark compartment'). From this point on, the time spend in either one of the compartments and the number of crossings was measured over a period of $5 \mathrm{~min}$.

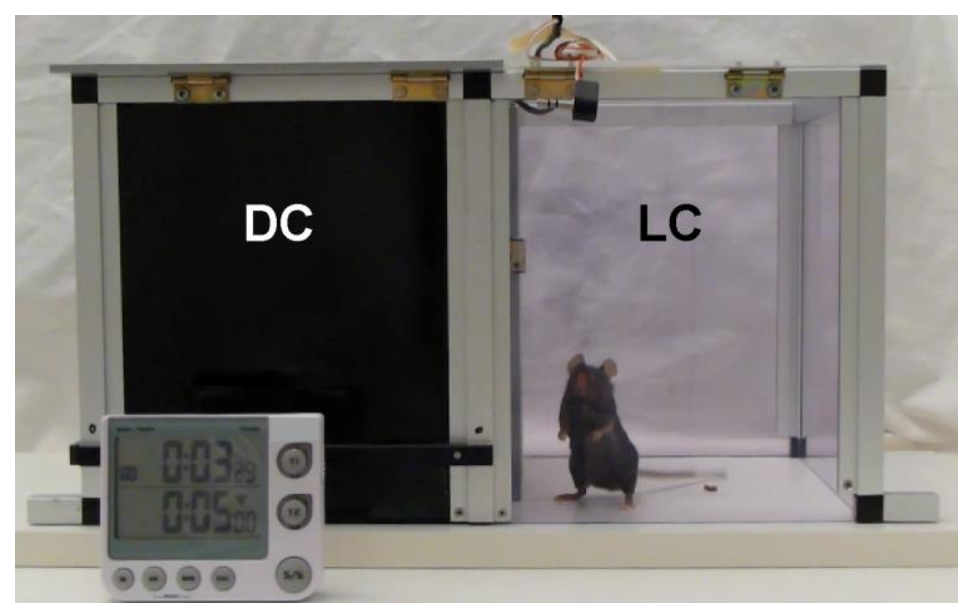

Fig. 37: Light-Dark preference test setup. Light (LC) and dark compartment (DC) are connected by a small door. 


\subsubsection{Y-maze spontaneous alternation test}

The Y-maze spontaneous alternation test was used to measure working memory deficits in transgenic mice. Because of the inate drive of a rodent to investigate a new enviroment, mice will explore a new arm of the $\mathrm{Y}$-maze rather than returning to one that was previously visited. Thus, over the course of multiple arm entries, normal mice show a tendency to more likely enter a less recently visited arm. Many parts of the brain, including the hippocampus, septum, basal forebrain, and prefrontal cortex, are involved in this task.

The test apparatus consisted of a three armed Y-shaped maze (at an angle of $120^{\circ}$ ) (Fig. 38). A mouse was introduced into the starting arm and was allowed to freely explore the maze for $10 \mathrm{~min}$. The experiment was recorded with an overhead video camera and the number of arm entries and their order was scored to calculate the percentage of alternation. An entry to an arm was counted when all four limbs were within the arm.

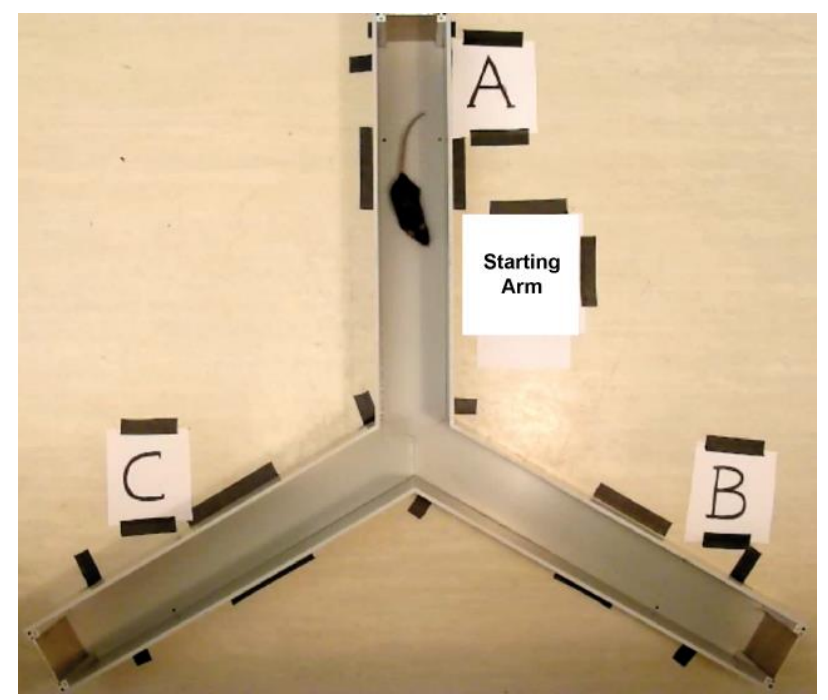

Fig. 38: Y-maze spontaneous alternation test setup. Mize were introduced into the starting arm and were allowed to freely explore the maze for $10 \mathrm{~min}$ with overhead video recording.

\subsubsection{Tail suspension test}

The tail suspension test is used assess moot levels and motivational behavior in rodents. Mice were hung by the tail for 6 min (Fig. 39) and the time in which they actively tried to get free or hung passively was quantified. Mice with high motivation were expected to fight longer to escape the uncomfortable situation. Mice, which managed to climb up their tail were excluded from the analysis. 


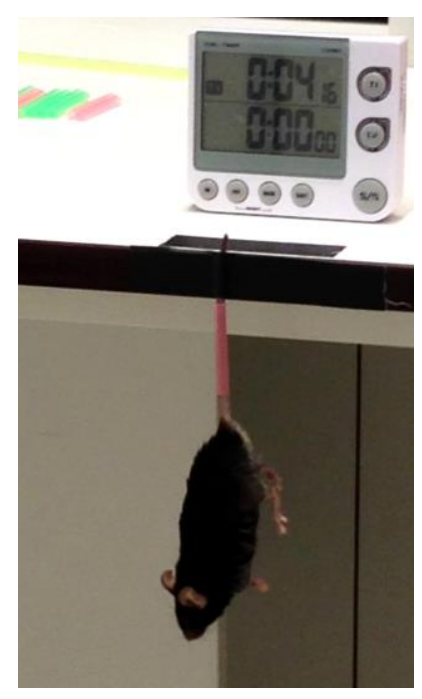

Fig. 39: Tail suspension test. Mice are hung by the tail for 6 min to access motivational states.

\subsubsection{Prepulse inhibition test}

Prepulse inhibition is phenomenon in which under normal conditions a weaker prestimulus inhibits the reaction to a subsequent strong startling stimulus. The reduction of the amplitude of startle reflects the ability of the nervous system to temporarily adapt to a strong sensory stimulus when a preceeding weaker signal is given to warn the organism. Deficits in prepulse inhibition manifest in the inability to filter out the unnecessary information, have been linked to abnormalities of sensorimotor gating and are well described in schizophrenia (Geyer and Braff, 1982; Braff et al., 1992).

The startle response was measured using San Diego Instruments' SR-Lab startle response system. Mice were placed into a tubular enclosure on a detector platform in the test chamber, recording vertical movements of the enclosure (Fig. 40). The startle reaction to an acoustic stimulus (body muscles contraction, jumping) evoking a movement of the platform and a transient force, was recorded with a computer during a time window of $100 \mathrm{~ms}$ (beginning with the onset of the acoustic stimulus) and stored for further evaluation. Since restraining is stressful for mice and could influence the read-out, animals were habituated with similar enclosures for at least 5 days prior to the experiment. With two experimental setups, always two mice were recorded at a time. It was ensured that genotypes always altered between the recorded pairs and setups. The protocol used was based on the protocol published in Brzózka et al. (2010). Briefly, the test started with an initial two minutes habituation phase to $65 \mathrm{~dB}$ background white noise (continuous throughout the session), followed by baseline recording for $1 \mathrm{~min}$. Startle reflexes were evoked by acoustic stimuli delivered from a loudspeaker. After the baseline recordings, six pulse-alone trials using startle stimuli of $120 \mathrm{~dB}$ intensity and $40 \mathrm{~ms}$ duration were applied to 
decrease influence of within-session habituation and scale down the initial startle response to a stable plateau. For tests of prepulse inhibition, the $120 \mathrm{~dB}$ startle pulse of $40 \mathrm{~ms}$ duration was applied either alone or preceeded by a non-startling prepulse stimulus of 70,75 or $80 \mathrm{~dB}$ intensity and $20 \mathrm{~ms}$ duration. An interval of $100 \mathrm{~ms}$ with background white noise was employed between each prepulse and pulse stimulus. The trials were presented in a pseudorandom order with inter-trial intervals ranging from 8 to $22 \mathrm{~s}$. The amplitude of the startle response (expressed in arbitrary units, AUs) was defined as the difference between the maximum force detected during a recording window and the force measured immediately before the stimulus onset. Maximum amplitudes were averaged for each individual animal, separately for all types of trials (i.e. stimulus alone or stimulus preceded by a prepulse). Prepulse inhibition (expressed in \%) was calculated as the percentage of the startle response using the following formula:

Prepulse inhibition $(\%)=100-\frac{\text { (startle amplitude after prepulse and pulse })}{\text { startle amplitude after pulse only }) \times 100}$

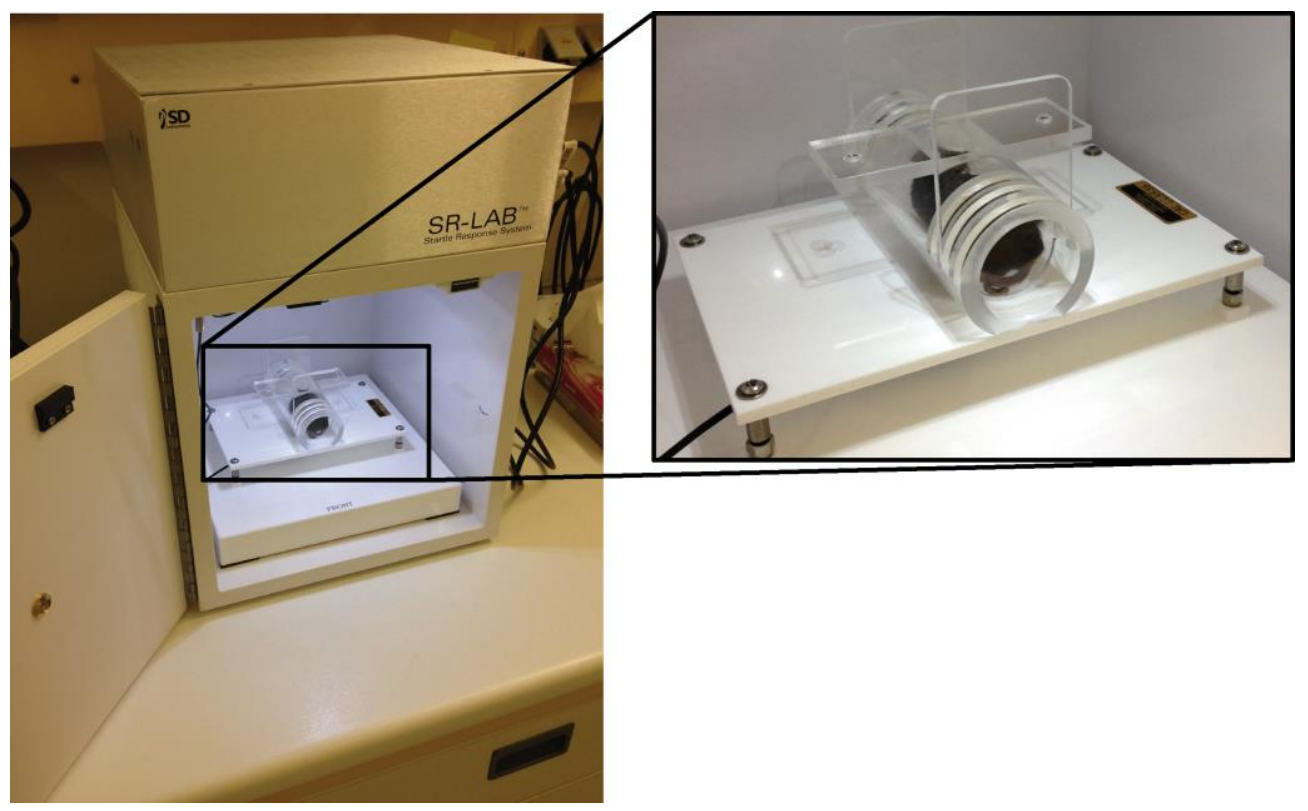

Fig. 40: San Diego Instruments' SR-Lab startle response system. Mice were placed into plastic restrainers on a detector platform recording vertical movements of the enclosure.

\subsubsection{Fear conditioning}

In this study two cohorts of conditional NRG1 transgenic mice were tested individually in the fear conditioning paradigm. In between these two tests a new fear conditioning setup was aquired, hence the Stop-Nrg $1^{*} \mathrm{CKII}$-Cre cohort was tested in 
the old TSE Systems setup, while the Stop-Nrg1*NEX-Cre cohort was tested in the new Ugo Basile Series 46000 Fear Conditioning System. The basic principle of the experiment was similar for both setups, the differences between the two will be explained as follows.

In this experiment the freezing behavior, defined as a lack of movement (excluding respiratory movements of the chest), is considered as a measure of memory. The experiment consists of three experimental trials, conducted over the course of three consecutive days. On the first day the mice were conditioned in the conditioning chamber. In the TSE Sytems setup the conditioning took place in a plexiglas conditioning chamber $(36 \times 20 \times 20 \mathrm{~cm})$ with a loudspeaker, a lamp (12 V) and a removable shock grid floor made of stainless rods $(4 \mathrm{~mm}$ diameter, spaced $6 \mathrm{~mm}$ apart). The grid was connected to the shock-scrambler unit applying an electrical shock of defined intensity and duration. The system had an overhead video camera connected to a monitor (Fig. 41C). The Ugo Basile setup, had a square conditioning box with a $15 \times 15 \mathrm{~cm}$ grid floor. In contrast to the TSE Systems setup, the walls of the chamber were covered with black and white striped pannels and the chamber was placed in a soundproof box with an infrared overhead camera (Fig. 41A). In the TSE System only one mouse was tested at a time, while in the Ugo Basile setup, two mice were tested at a time, in two setups. Here a white noise tone was played during the experiment.

The training for the contextual and cued fear memory took place at the same time. A mouse was placed into the conditioning box and its behavior (freezing rate) was observed as an initial baseline phase of $2 \mathrm{~min}$. In the TSE System setup, freezing was scored every $4 \mathrm{~s}$ during 2 min (context baseline) by the blind to genotype experimenter, while in the Ugo Basile setup the freezing events and durations were scored by the software, in both experiments the whole session was recorded by a camera for later rescoring. After the 2 min pre-exposure time (=context baseline) a tone was played for $30 \mathrm{sec}$ (conditioned stimulus (CS); $10 \mathrm{kHz}, 75 \mathrm{~dB}$ ). Directly after the tone a mild electrical foot shock was applied (0.4 mA, $2 \mathrm{~s}$ duration). After a 30 sec pause the tone and shock were repeated with same intensity and duration. After the second foot shock each mouse was allowed to stay in the conditioning box for 30 sec to avoid the association of aversive stimuli with the experimenter. Afterwards animals were placed back into their home cages. The conditioning box was cleaned with $70 \%$ ethanol p.A. between trials. On the second day, contextual fear memory was assessed by scoring the freezing response for $2 \mathrm{~min}$ in the conditioning box in the absence of tone and shock. Another $24 \mathrm{~h}$ later mice were placed into a new ('non-context') box. In the TSE Systems experiment this box was a triangular grey 
plastic chamber $(36 \times 20 \times 20 \times 20 \mathrm{~cm}$ ) that was placed onto a different table, while the conditioning chamber was covered by white sheets (Fig. 41D). In the Ugo Basile experiment a transparent plastic cylinder $(36 \times 20 \mathrm{~cm})$ was placed onto a grey plastic floor into the soundproof box (Fig. 41B). Baseline freezing behavior was scored for 2 min, followed by 2 min of the cue tone (CS) and scoring of freezing response as a measure of cued fear memory. The 'cue' box was cleaned with water between trials to avoid olfactory cues resembling conditioning. In both cases the whole experiment was video recorded for second rescoring.

A

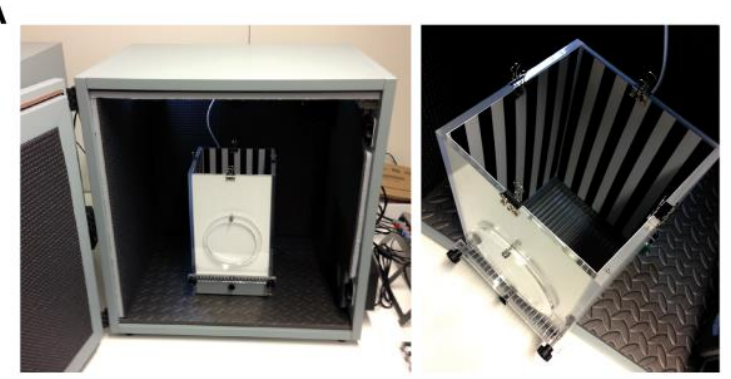

C

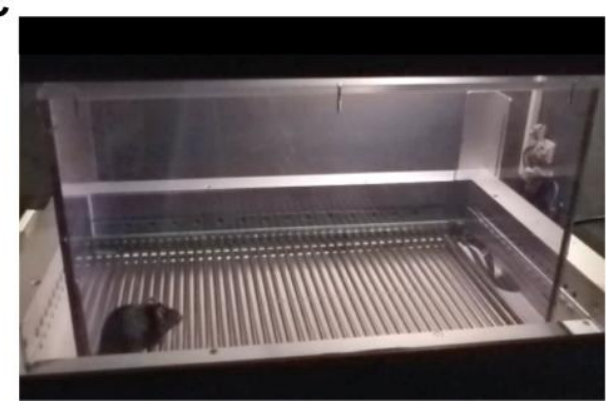

B

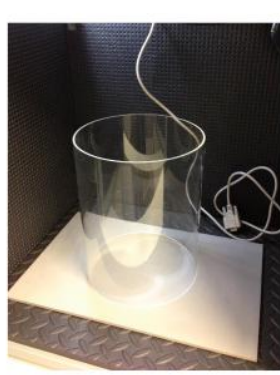

D

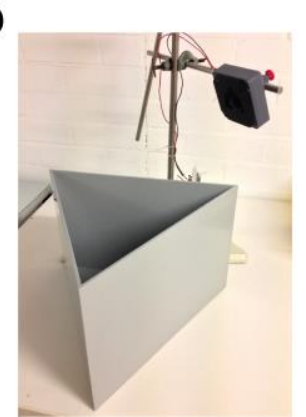

Fig. 41: Ugo Basile Series 46000 and TSE Systems fear conditioning setups. The Ugo Basile system used a conditioning and contextual fear memory box with grid and striped walls (A) and a round plastic cylinder on a grey floor for cued fear memory (B) inside a soundproof test apparatus. In the TSE Systems setup a bigger gridded box was used for conditioning and assessment of contextual fear memory (C), while the cued fear memory was measured in a triangular box on a separat table (D). 


\section{References}

Ackermann M, Matus A (2003) Activity-induced targeting of profilin and stabilization of dendritic spine morphology. Nat Neurosci 6:1194-1200.

Agarwal A et al. (2014) Dysregulated expression of neuregulin-1 by cortical pyramidal neurons disrupts synaptic plasticity. CellReports 8:1130-1145.

Agarwal A, Dibaj P, Kassmann CM, Goebbels S, Nave K-A, Schwab MH (2011) In Vivo Imaging and Noninvasive Ablation of Pyramidal Neurons in Adult NEX-CreERT2 Mice. Cereb cortex (New York, NY 1991).

Akbarian S, Kim JJ, Potkin SG, Hagman JO, Tafazzoli A, Bunney WE, Jones EG (1995) Gene expression for glutamic acid decarboxylase is reduced without loss of neurons in prefrontal cortex of schizophrenics. Arch Gen Psychiatry 52:258-266.

Allen NJ, Barres BA (2009) Neuroscience: Glia - more than just brain glue. Nature 457:675677.

Andreasen NC (1995) Symptoms, signs, and diagnosis of schizophrenia. Lancet 346:477481.

Arasada RR, Carpenter G (2005) Secretase-dependent tyrosine phosphorylation of Mdm2 by the ErbB-4 intracellular domain fragment. J Biol Chem 280:30783-30787.

Arber S, Barbayannis FA, Hanser H, Schneider C, Stanyon CA, Bernard O, Caroni P (1998) Regulation of actin dynamics through phosphorylation of cofilin by LIM-kinase. Nature 393:805-809.

Ayalew M et al. (2012) Convergent functional genomics of schizophrenia: from comprehensive understanding to genetic risk prediction. Mol Psychiatry.

Baldan Ramsey LC, Xu M, Wood N, Pittenger C (2011) Lesions of the dorsomedial striatum disrupt prepulse inhibition. Neuroscience 180:222-228.

Bao J, Lin H, Ouyang Y, Lei D, Osman A, Kim TW, Mei L, Dai P, Ohlemiller KK, Ambron RT (2004) Activity-dependent transcription regulation of PSD-95 by neuregulin-1 and Eos. Nat Neurosci 7:1250-1258.

Bao J, Wolpowitz D, Role LW, Talmage DA (2003) Back signaling by the Nrg-1 intracellular domain. Sci STKE 161:1133.

Barnes AP, Polleux F (2009) Establishment of axon-dendrite polarity in developing neurons. Annu Rev Neurosci 32:347-381.

Barros CS, Calabrese B, Chamero P, Roberts AJ, Korzus E, Lloyd K, Stowers L, Mayford M, Halpain S, Müller U (2009) Impaired maturation of dendritic spines without disorganization of cortical cell layers in mice lacking NRG1/ErbB signaling in the central nervous system. Proc Natl Acad Sci U S A 106:4507-4512.

Bartos M, Vida I, Jonas P (2007) Synaptic mechanisms of synchronized gamma oscillations in inhibitory interneuron networks. Nat Rev Neurosci 8:45-56.

Bean JC, Lin TW, Sathyamurthy A, Liu F, Yin D-M, Xiong W-C, Mei L (2014) Genetic Labeling Reveals Novel Cellular Targets of Schizophrenia Susceptibility Gene: Distribution of GABA and Non-GABA ErbB4-Positive Cells in Adult Mouse Brain. J Neurosci 34:13549-13566.

Benes FM, Berretta S (2001) GABAergic interneurons: implications for understanding schizophrenia and bipolar disorder. Neuropsychopharmacology 25:1-27.

Benes FM, McSparren J, Bird ED, SanGiovanni JP, Vincent SL (1991) Deficits in small interneurons in prefrontal and cingulate cortices of schizophrenic and schizoaffective patients. Arch Gen Psychiatry 48:996-1001.

Bertram I, Bernstein H-G, Lendeckel U, Bukowska A, Dobrowolny H, Keilhoff G, Kanakis D, Mawrin C, Bielau H, Falkai P, Bogerts B (2007) Immunohistochemical evidence for impaired neuregulin-1 signaling in the prefrontal cortex in schizophrenia and in unipolar depression. Ann N Y Acad Sci 1096:147-156. 
Biesemann C, Grønborg M, Luquet E, Wichert SP, Bernard V, Bungers SR, Cooper B, Varoqueaux F, Li L, Byrne JA, Urlaub H, Jahn O, Brose N, Herzog E (2014) Proteomic screening of glutamatergic mouse brain synaptosomes isolated by fluorescence activated sorting. EMBO J 33:157-170.

Birchmeier C, Nave K-A (2008) Neuregulin-1, a key axonal signal that drives Schwann cell growth and differentiation. Glia 56:1491-1497.

Bishop C, Walker PD (2003) Combined intrastriatal dopamine D1 and serotonin 5-HT2 receptor stimulation reveals a mechanism for hyperlocomotion in 6-hydroxydopaminelesioned rats. Neuroscience 121:649-657.

Bjarnadottir M, Misner DL, Haverfield-Gross S, Bruun S, Helgason VG, Stefansson H, Sigmundsson A, Firth DR, Nielsen B, Stefansdottir R, Novak TJ, Stefansson K, Gurney ME, Andresson T (2007) Neuregulin1 (NRG1) signaling through Fyn modulates NMDA receptor phosphorylation: differential synaptic function in NRG1+/- knock-outs compared with wild-type mice. J Neurosci 27:4519-4529.

Black JA, Waxman SG (1988) The perinodal astrocyte. Glia 1:169-183.

Bradley DW, Joyce D, Murphy EH, Nash BM, Porsolt RD, Summerfield A, Twyman WA (1968) Amphetamine-barbiturate mixture: effects on the behaviour of mice. Nature 220:187-188.

Braff DL, Geyer MA (1990) Sensorimotor gating and schizophrenia. Human and animal model studies. Arch Gen Psychiatry 47:181-188.

Braff DL, Geyer MA, Swerdlow NR (2001) Human studies of prepulse inhibition of startle: normal subjects, patient groups, and pharmacological studies. Psychopharmacology (Berl) 156:234-258.

Braff DL, Grillon C, Geyer MA (1992) Gating and habituation of the startle reflex in schizophrenic patients. Arch Gen Psychiatry 49:206-215.

Brinkmann BG, Agarwal A, Sereda MW, Garratt AN, Müller T, Wende H, Stassart RM, Nawaz $S$, Humml C, Velanac $V(2008)$ Neuregulin-1/ErbB signaling serves distinct functions in myelination of the peripheral and central nervous system. Neuron 59:581-595.

Britto JM, Lukehurst S, Weller R, Fraser C, Qu Y, Hertzog P, Busfield SJ (2004) Generation and characterization of neuregulin-2-deficient mice. Mol Cell Biol 24:8221-8226.

Broadhurst PL (1961) Analysis of maternal effects in the inheritance of behaviour. Anim Behav 9:129-141.

Brose N (1999) Synaptic cell adhesion proteins and synaptogenesis in the mammalian central nervous system. Naturwissenschaften 86:516-524.

Brus R, Nowak P, Szkilnik R, Mikolajun U, Kostrzewa RM (2004) Serotoninergics attenuate hyperlocomotor activity in rats. Potential new therapeutic strategy for hyperactivity. Neurotox Res 6:317-325.

Brzózka MM, Radyushkin K, Wichert SP, Ehrenreich H, Rossner MJ (2010) Cognitive and sensorimotor gating impairments in transgenic mice overexpressing the schizophrenia susceptibility gene Tcf4 in the brain. Biol Psychiatry 68:33-40.

Buac K, Xu M, Cronin J, Weeraratna AT, Hewitt SM, Pavan WJ (2009) NRG1 / ERBB3 signaling in melanocyte development and melanoma: inhibition of differentiation and promotion of proliferation. Pigment Cell Melanoma Res 22:773-784.

Burgess AW, Cho H-S, Eigenbrot C, Ferguson KM, Garrett TPJ, Leahy DJ, Lemmon MA, Sliwkowski MX, Ward CW, Yokoyama S (2003) An open-and-shut case? Recent insights into the activation of EGF/ErbB receptors. Mol Cell 12:541-552.

Burgess TL, Ross SL, Qian YX, Brankow D, Hu S (1995) Biosynthetic processing of neu differentiation factor. Glycosylation trafficking, and regulated cleavage from the cell surface. J Biol Chem 270:19188-19196.

Buxbaum JD, Georgieva L, Young JJ, Plescia C, Kajiwara Y, Jiang Y, Moskvina V, Norton N, Peirce T, Williams H, Craddock NJ, Carroll L, Corfas G, Davis KL, Owen MJ, Harroch S, Sakurai T, O'Donovan MC (2008) Molecular dissection of NRG1-ERBB4 signaling 
implicates PTPRZ1 as a potential schizophrenia susceptibility gene. Mol Psychiatry 13:162-172.

Cahill ME, Remmers C, Jones KA, Xie Z, Sweet RA, Penzes P (2013) Neuregulin1 signaling promotes dendritic spine growth through kalirin. J Neurochem.

Cahill ME, Xie Z, Day M, Photowala H, Barbolina M V, Miller CA, Weiss C, Radulovic J, Sweatt JD, Disterhoft JF, Surmeier DJ, Penzes P (2009) Kalirin regulates cortical spine morphogenesis and disease-related behavioral phenotypes. Proc Natl Acad Sci U S A 106:13058-13063.

Campsall KD, Mazerolle CJ, De Repentingy Y, Kothary R, Wallace VA (2002) Characterization of transgene expression and Cre recombinase activity in a panel of Thy-1 promoter-Cre transgenic mice. Dev Dyn 224:135-143.

Carlini VP, Varas MM, Cragnolini AB, Schiöth HB, Scimonelli TN, de Barioglio SR (2004) Differential role of the hippocampus, amygdala, and dorsal raphe nucleus in regulating feeding, memory, and anxiety-like behavioral responses to ghrelin. Biochem Biophys Res Commun 313:635-641.

Carraway KL, Weber JL, Unger MJ, Ledesma J, Yu N, Gassmann M, Lai C (1997) Neuregulin-2, a new ligand of ErbB3/ErbB4-receptor tyrosine kinases. Nature 387:512516.

Chance SA, Walker M, Crow TJ (2005) Reduced density of calbindin-immunoreactive interneurons in the planum temporale in schizophrenia. Brain Res 1046:32-37.

Chen Y, Hancock ML, Role LW, Talmage DA (2010) Intramembranous valine linked to schizophrenia is required for neuregulin 1 regulation of the morphological development of cortical neurons. J Neurosci 30:9199-9208.

Chen Y-JJ, Johnson MA, Lieberman MD, Goodchild RE, Schobel S, Lewandowski N, Rosoklija G, Liu R-C, Gingrich JA, Small S, Moore H, Dwork AJ, Talmage DA, Role LW (2008) Type III neuregulin-1 is required for normal sensorimotor gating, memory-related behaviors, and corticostriatal circuit components. J Neurosci 28:6872-6883.

Cheng Q-C, Tikhomirov O, Zhou W, Carpenter G (2003) Ectodomain cleavage of ErbB-4: characterization of the cleavage site and m80 fragment. J Biol Chem 278:38421-38427.

Cho KO, Hunt CA, Kennedy MB (1992) The rat brain postsynaptic density fraction contains a homolog of the Drosophila discs-large tumor suppressor protein. Neuron 9:929-942.

Chong VZ, Thompson M, Beltaifa S, Webster MJ, Law AJ, Shannon Weickert CS (2008) Elevated neuregulin-1 and ErbB4 protein in the prefrontal cortex of schizophrenic patients. Schizophr Res 100:270-280.

Citri A, Skaria KB, Yarden Y (2003) The deaf and the dumb: the biology of ErbB-2 and ErbB3. Exp Cell Res 284:54-65.

Crawley J, Goodwin FK (1980) Preliminary report of a simple animal behavior model for the anxiolytic effects of benzodiazepines. Pharmacol Biochem Behav 13:167-170.

Crawley JN (2007) What's Wrong With My Mouse: Behavioral Phenotyping of Transgenic and Knockout Mice. Wiley.

Deakin IH, Law AJ, Oliver PL, Schwab MH, Nave K-A, Harrison PJ, Bannerman DM (2009) Behavioural characterization of neuregulin 1 type I overexpressing transgenic mice. Neuroreport 20:1523-1528.

Deakin IH, Nissen W, Law AJ, Lane T, Kanso R, Schwab MH, Nave K-A, Lamsa KP, Paulsen O, Bannerman DM, Harrison PJ (2012) Transgenic Overexpression of the Type I Isoform of Neuregulin 1 Affects Working Memory and Hippocampal Oscillations but not Long-term Potentiation. Cereb cortex (New York, NY 1991) 22:1520-1529.

DeFelipe $J$ et al. (2013) New insights into the classification and nomenclature of cortical GABAergic interneurons. Nat Rev Neurosci.

Del Pino I, García-Frigola C, Dehorter N, Brotons-Mas JR, Alvarez-Salvado E, Martínez de Lagrán M, Ciceri G, Gabaldón MV, Moratal D, Dierssen M, Canals S, Marin O, Rico B 
(2013) Erbb4 deletion from fast-spiking interneurons causes schizophrenia-like phenotypes. Neuron 79:1152-1168.

Diamonti AJ, Guy PM, Ivanof C, Wong K, Sweeney C, Carraway KL (2002) An RBCC protein implicated in maintenance of steady-state neuregulin receptor levels. Proc Natl Acad Sci U S A 99:2866-2871.

Edwards DC, Sanders LC, Bokoch GM, Gill GN (1999) Activation of LIM-kinase by Pak1 couples Rac/Cdc42 GTPase signalling to actin cytoskeletal dynamics. Nat Cell Biol $1: 253-259$.

Elenius K, Choi CJ, Paul S, Santiestevan E, Nishi E, Klagsbrun M (1999) Characterization of a naturally occurring ErbB4 isoform that does not bind or activate phosphatidyl inositol 3-kinase. Oncogene 18:2607-2615.

Elenius K, Corfas G, Paul S, Choi CJ, Rio C, Plowman GD, Klagsbrun M (1997) A novel juxtamembrane domain isoform of HER4/ErbB4. Isoform-specific tissue distribution and differential processing in response to phorbol ester. J Biol Chem 272:26761-26768.

Emamian ES, Hall D, Birnbaum MJ, Karayiorgou M, Gogos JA (2004) Convergent evidence for impaired AKT1-GSK3beta signaling in schizophrenia. Nat Genet 36:131-137.

Ennequin G, Boisseau N, Caillaud K, Chavanelle V, Etienne M, Li X, Montaurier C, Sirvent P (2015) Neuregulin 1 affects leptin levels, food intake and weight gain in normal-weight, but not obese, $\mathrm{db} / \mathrm{db}$ mice. Diabetes Metab.

Fadda P, Scherma M, Fresu A, Collu M, Fratta W (2005) Dopamine and serotonin release in dorsal striatum and nucleus accumbens is differentially modulated by morphine in DBA/2J and C57BL/6J mice. Synap (New York, NY) 56:29-38.

Falls DL (2003) Neuregulins: functions, forms, and signaling strategies. Exp Cell Res 284:1430.

Farzan F, Barr MS, Levinson AJ, Chen R, Wong W, Fitzgerald PB, Daskalakis ZJ (2010) Evidence for gamma inhibition deficits in the dorsolateral prefrontal cortex of patients with schizophrenia. Brain 133:1505-1514.

Fazzari P, Paternain A V, Valiente M, Pla R, Luján R, Lloyd K, Lerma J, Marin O, Rico B (2010) Control of cortical GABA circuitry development by Nrg1 and ErbB4 signalling. Nature 464:1376-1380.

Fazzari P, Snellinx A, Sabonov V, Ahmed T, Serneels L, Gartner A, Shariati SAM, Balschun D, De Strooper B (2014) Cell autonomous regulation of hippocampal circuitry via Aph1b$\mathrm{Y}$-secretase/Neuregulin 1 signalling. Elife:e02196.

Feil R, Wagner J, Metzger D, Chambon P (1997) Regulation of Cre recombinase activity by mutated estrogen receptor ligand-binding domains. Biochem Biophys Res Commun 237:752-757.

Flames N, Long JE, Garratt AN, Fischer TM, Gassmann M, Birchmeier C, Lai C, Rubenstein JLR, Marin O (2004) Short- and long-range attraction of cortical GABAergic interneurons by neuregulin-1. Neuron 44:251-261.

Fleck D, van Bebber F, Colombo A, Galante C, Schwenk BM, Rabe L, Hampel H, Novak B, Kremmer E, Tahirovic S, Edbauer D, Lichtenthaler SF, Schmid B, Willem M, Haass C (2013) Dual Cleavage of Neuregulin 1 Type III by BACE1 and ADAM17 Liberates Its EGF-Like Domain and Allows Paracrine Signaling. J Neurosci 33:7856-7869.

Freund TF, Buzsáki G (1996) Interneurons of the hippocampus. Hippocampus 6:347-470.

Fu AK, Fu WY, Cheung J, Tsim KW, Ip FC, Wang JH, Ip NY (2001) Cdk5 is involved in neuregulin-induced AChR expression at the neuromuscular junction. Nat Neurosci 4:374-381.

Funfschilling U, Supplie LM, Mahad D, Boretius S, Saab AS, Edgar J, Brinkmann BG, Kassmann CM, Tzvetanova ID, Möbius W, Diaz F, Meijer D, Suter U, Hamprecht B, Sereda MW, Moraes CT, Frahm J, Goebbels S, Nave K-A (2012) Glycolytic oligodendrocytes maintain myelin and long-term axonal integrity. Nature 485:517-521. 
Garbay B, Heape AM, Sargueil F, Cassagne C (2000) Myelin synthesis in the peripheral nervous system. Prog Neurobiol 61:267-304.

Garcia RA, Vasudevan K, Buonanno A (2000) The neuregulin receptor ErbB-4 interacts with PDZ-containing proteins at neuronal synapses. Proc Natl Acad Sci U S A 97:35963601.

Garcia-Barcelo M-M et al. (2009) Genome-wide association study identifies NRG1 as a susceptibility locus for Hirschsprung's disease. Proc Natl Acad Sci U S A 106:26942699.

Gärtner U, Alpár A, Behrbohm J, Heumann R, Arendt T (2005) Enhanced Ras activity promotes spine formation in synRas mice neocortex. Neuroreport 16:149-152.

Gassmann M, Casagranda F, Orioli D, Simon H, Lai C, Klein R, Lemke G (1995) Aberrant neural and cardiac development in mice lacking the ErbB4 neuregulin receptor. Nature 378:390-394.

Geyer MA, Braff DL (1982) Habituation of the Blink reflex in normals and schizophrenic patients. Psychophysiology 19:1-6.

Giedd JN, Blumenthal J, Jeffries NO, Castellanos FX, Liu H, Zijdenbos A, Paus T, Evans AC, Rapoport JL (1999) Brain development during childhood and adolescence: a longitudinal MRI study. Nat Neurosci 2:861-863.

Goebbels S, Bormuth I, Bode U, Hermanson O, Schwab MH, Nave K-A (2006) Genetic targeting of principal neurons in neocortex and hippocampus of NEX-Cre mice. Genes (New York, NY 2000) 44:611-621.

Golub MS, Germann SL, Lloyd KCK (2004) Behavioral characteristics of a nervous systemspecific erbB4 knock-out mouse. Behav Brain Res 153:159-170.

Goring DR, Rossant J, Clapoff S, Breitman ML, Tsui LC (1987) In situ detection of betagalactosidase in lenses of transgenic mice with a gamma-crystallin/lacZ gene. Sci (New York, NY) 235:456-458.

Gorski JA, Talley T, Qiu M, Puelles L, Rubenstein JLR, Jones KR (2002) Cortical excitatory neurons and glia, but not GABAergic neurons, are produced in the Emx1-expressing lineage. J Neurosci 22:6309-6314.

Gottesman II (1991) Schizophrenia genesis: The origins of madness.

Gu Z, Jiang Q, Fu AKY, Ip NY, Yan Z (2005) Regulation of NMDA receptors by neuregulin signaling in prefrontal cortex. J Neurosci 25:4974-4984.

Gui H, Tang W-K, So M-T, Proitsi P, Sham PC, Tam PK, Sau-Wai Ngan E, Cherny SS, Garcia-Barceló M-M (2013) RET and NRG1 interplay in Hirschsprung disease. Hum Genet.

Guy PM, Platko J V, Cantley LC, Cerione RA, Carraway KL (1994) Insect cell-expressed p180erbB3 possesses an impaired tyrosine kinase activity. Proc Natl Acad Sci U S A 91:8132-8136.

Hahn C-G, Wang H-Y, Cho D-S, Talbot K, Gur RE, Berrettini WH, Bakshi K, Kamins J, Borgmann-Winter KE, Siegel SJ, Gallop RJ, Arnold SE (2006) Altered neuregulin 1erbB4 signaling contributes to NMDA receptor hypofunction in schizophrenia. Nat Med 12:824-828.

Hall CS (1934) Emotional behavior in the rat. I. Defecation and urination as measures of individual differences in emotionality. J Comp Psychol 18:385-403.

Harlow E, Lane D (1988) Antibodies: a laboratory manual. Cold Spring Harbor Laboratory.

Harrison PJ (1999) The neuropathology of schizophrenia. A critical review of the data and their interpretation. Brain 122 ( Pt 4:593-624.

Harrison PJ (2015) Recent genetic findings in schizophrenia and their therapeutic relevance. J Psychopharmacol 29:85-96.

Harrison PJ, Law AJ (2006) Neuregulin 1 and schizophrenia: genetics, gene expression, and neurobiology. Biol Psychiatry 60:132-140. 
Harrison PJ, Weinberger DR (2005) Schizophrenia genes, gene expression, and neuropathology: on the matter of their convergence. Mol Psychiatry 10:40-68; image 5.

Hashimoto T, Volk DW, Eggan SM, Mirnics K, Pierri JN, Sun Z, Sampson AR, Lewis DA (2003) Gene expression deficits in a subclass of GABA neurons in the prefrontal cortex of subjects with schizophrenia. J Neurosci 23:6315-6326.

Hatzimanolis A, Mcgrath JA, Wang R, Li T, Wong PC, Nestadt G, Wolyniec PS, Valle D, Pulver AE, Avramopoulos D (2013) Multiple variants aggregate in the neuregulin signaling pathway in a subset of schizophrenia patients. Transl Psychiatry 3:e264.

HEBB CO, WHITTAKER VP (1958) Intracellular distributions of acetylcholine and choline acetylase. J Physiol 142:187-196.

Heckers S, Konradi C (2014) GABAergic mechanisms of hippocampal hyperactivity in schizophrenia. Schizophr Res.

Hell JW, Jahn R (2006) Chapter 12 - Preparation of Synaptic Vesicles from Mammalian Brain. In: Cell Biology (Third Edition), Third Edit. (Celis JE, ed), pp 85-90. Burlington: Academic Press.

Ho WH, Armanini MP, Nuijens A, Phillips HS, Osheroff PL (1995) Sensory and motor neuronderived factor. A novel heregulin variant highly expressed in sensory and motor neurons. J Biol Chem 270:14523-14532.

Holmes WE, Sliwkowski MX, Akita RW, Henzel WJ, Lee J, Park JW, Yansura D, Abadi N, Raab H, Lewis GD (1992) Identification of heregulin, a specific activator of p185erbB2. Science (80- ) 256:1205-1210.

Holt DJ, Bachus SE, Hyde TM, Wittie M, Herman MM, Vangel M, Saper CB, Kleinman JE (2005) Reduced density of cholinergic interneurons in the ventral striatum in schizophrenia: an in situ hybridization study. Biol Psychiatry 58:408-416.

Horiuchi K, Zhou H-M, Kelly K, Manova K, Blobel CP (2005) Evaluation of the contributions of ADAMs $9,12,15,17$, and 19 to heart development and ectodomain shedding of neuregulins beta1 and beta2. Dev Biol 283:459-471.

Hu X, He W, Diaconu C, Tang X, Kidd GJ, Macklin WB, Trapp BD, Yan R (2008) Genetic deletion of BACE1 in mice affects remyelination of sciatic nerves. FASEB J 22:29702980.

Hu X, Hicks CW, He W, Wong P, Macklin WB, Trapp BD, Yan R (2006) Bace1 modulates myelination in the central and peripheral nervous system. Nat Neurosci 9:1520-1525.

Huang YZ, Won S, Ali DW, Wang Q, Tanowitz M, Du QS, Pelkey KA, Yang DJ, Xiong W-C, Salter MW, Mei L (2000) Regulation of neuregulin signaling by PSD-95 interacting with ErbB4 at CNS synapses. Neuron 26:443-455.

Huijbregts RPH, Roth KA, Schmidt RE, Carroll SL (2003) Hypertrophic neuropathies and malignant peripheral nerve sheath tumors in transgenic mice overexpressing glial growth factor beta3 in myelinating Schwann cells. J Neurosci 23:7269-7280.

Huttenlocher PR (1979) Synaptic density in human frontal cortex - developmental changes and effects of aging. Brain Res 163:195-205.

Huttner WB, Schiebler W, Greengard P, De Camilli P (1983) Synapsin I (protein I), a nerve terminal-specific phosphoprotein. III. Its association with synaptic vesicles studied in a highly purified synaptic vesicle preparation. J Cell Biol 96:1374-1388.

Jablensky A (1995) Schizophrenia: recent epidemiologic issues. Epidemiol Rev 17:10-20.

Jiang L, O'Leary C, Kim HA, Parish CL, Massalas J, Waddington JL, Ehrlich ME, Schütz G, Gantois I, Lawrence AJ, Drago J (2015) Motor and behavioral phenotype in conditional mutants with targeted ablation of cortical D1 dopamine receptor-expressing cells. Neurobiol Dis 76:137-158.

Jones EG (1998) Viewpoint: the core and matrix of thalamic organization. Neuroscience 85:331-345.

Jones JT, Akita RW, Sliwkowski MX (1999) Binding specificities and affinities of egf domains for ErbB receptors. FEBS Lett 447:227-231. 
Junttila TT, Sundvall M, Määttä JA, Elenius K (2000) Erbb4 and its isoforms: selective regulation of growth factor responses by naturally occurring receptor variants. Trends Cardiovasc Med 10:304-310.

Kainulainen V, Sundvall M, Määttä JA, Santiestevan E, Klagsbrun M, Elenius K (2000) A natural ErbB4 isoform that does not activate phosphoinositide 3-kinase mediates proliferation but not survival or chemotaxis. J Biol Chem 275:8641-8649.

Kandel E (2013) Principles of Neural Science, Fifth Edition. McGraw-Hill Education.

Kaushansky A, Gordus A, Budnik BA, Lane WS, Rush J, Macbeath G (2008) System-wide investigation of ErbB4 reveals 19 sites of Tyr phosphorylation that are unusually selective in their recruitment properties. Chem Biol 15:808-817.

Kennedy JL (1996) Schizophrenia genetics: the quest for an anchor. Am J Psychiatry 153:1513-1514.

Kennedy MB (1997) The postsynaptic density at glutamatergic synapses. Trends Neurosci 20:264-268.

Kim E, Sheng M (2004) PDZ domain proteins of synapses. Nat Rev Neurosci 5:771-781.

Kistner U, Wenzel BM, Veh RW, Cases-Langhoff C, Garner AM, Appeltauer U, Voss B, Gundelfinger ED, Garner CC (1993) SAP90, a rat presynaptic protein related to the product of the Drosophila tumor suppressor gene dlg-A. J Biol Chem 268:4580-4583.

Klapper LN, Glathe S, Vaisman N, Hynes NE, Andrews GC, Sela M, Yarden Y (1999) The ErbB-2/HER2 oncoprotein of human carcinomas may function solely as a shared coreceptor for multiple stroma-derived growth factors. Proc Natl Acad Sci U S A 96:4995-5000.

Kohl S, Heekeren K, Klosterkötter J, Kuhn J (2013) Prepulse inhibition in psychiatric disorders--apart from schizophrenia. J Psychiatr Res 47:445-452.

Komuro A, Nagai M, Navin NE, Sudol M (2003) WW domain-containing protein YAP associates with ErbB-4 and acts as a co-transcriptional activator for the carboxylterminal fragment of ErbB-4 that translocates to the nucleus. J Biol Chem 278:3333433341.

Kwon O-B, Longart M, Vullhorst D, Hoffman DA, Buonanno A (2005) Neuregulin-1 reverses long-term potentiation at CA1 hippocampal synapses. J Neurosci 25:9378-9383.

Laemmli UK (1970) Cleavage of structural proteins during the assembly of the head of bacteriophage T4. Nature 227:680-685.

Lalo U, Andrew J, Palygin O, Pankratov Y (2009) Ca2+-dependent modulation of GABAA and NMDA receptors by extracellular ATP: implication for function of tripartite synapse. Biochem Soc Trans 37:1407-1411.

Law AJ, Kleinman JE, Weinberger DR, Shannon Weickert CS (2007) Disease-associated intronic variants in the ErbB4 gene are related to altered ErbB4 splice-variant expression in the brain in schizophrenia. Hum Mol Genet 16:129-141.

Law AJ, Lipska BK, Shannon Weickert CS, Hyde TM, Straub RE, Hashimoto R, Harrison PJ, Kleinman JE, Weinberger DR (2006) Neuregulin 1 transcripts are differentially expressed in schizophrenia and regulated by 5 ' SNPs associated with the disease. Proc Natl Acad Sci U S A 103:6747-6752.

Law AJ, Shannon Weickert CS, Hyde TM, Kleinman JE, Harrison PJ (2004) Neuregulin-1 (NRG-1) mRNA and protein in the adult human brain. Neuroscience 127:125-136.

Law AJ, Wang Y, Sei Y, O'Donnell P, Piantadosi P, Papaleo F, Straub RE, Huang W, Thomas CJ, Vakkalanka R, Besterman AD, Lipska BK, Hyde TM, Harrison PJ, Kleinman JE, Weinberger DR (2012) Neuregulin 1-ErbB4-PI3K signaling in schizophrenia and phosphoinositide 3-kinase-p110ס inhibition as a potential therapeutic strategy. Proc Natl Acad Sci U S A.

Leask SJ (2004) Environmental influences in schizophrenia: the known and the unknown. Adv Psychiatr Treat 10:323-330. 
Lee H-J, Jung K-M, Huang YZ, Bennett LB, Lee JS, Mei L, Kim T-W (2002) Presenilindependent gamma-secretase-like intramembrane cleavage of ErbB4. J Biol Chem 277:6318-6323.

Levitt $P$ (2005) Disruption of interneuron development. Epilepsia 46 Suppl 7:22-28.

Lewis DA, Hashimoto T, Volk DW (2005) Cortical inhibitory neurons and schizophrenia. Nat Rev Neurosci 6:312-324.

Li B, Woo R-S, Mei L, Malinow R (2007) The neuregulin-1 receptor erbB4 controls glutamatergic synapse maturation and plasticity. Neuron 54:583-597.

Li D, Collier DA, He L (2006) Meta-analysis shows strong positive association of the neuregulin 1 (NRG1) gene with schizophrenia. Hum Mol Genet 15:1995-2002.

Li K-X, Lu Y-M, Xu Z-H, Zhang J, Zhu J-M, Zhang J-M, Cao S-X, Chen X-J, Chen Z, Luo J-H, Duan S, Li X-M (2011) Neuregulin 1 regulates excitability of fast-spiking neurons through Kv1.1 and acts in epilepsy. Nat Neurosci.

Li L, Cleary S, Mandarano MA, Long W, Birchmeier C, Jones FE (2002) The breast protooncogene, HRGalpha regulates epithelial proliferation and lobuloalveolar development in the mouse mammary gland. Oncogene 21:4900-4907.

Linggi B, Carpenter G (2006) ErbB receptors: new insights on mechanisms and biology. Trends Cell Biol 16:649-656.

Liu X, Bates R, Yin D-M, Shen C, Wang F, Su N, Kirov SA, Luo Y, Wang J-Z, Xiong W-C, Mei L (2011) Specific Regulation of NRG1 Isoform Expression by Neuronal Activity. J Neurosci 31:8491-8501.

Liu Y, Tao Y-M, Woo R-S, Xiong W-C, Mei L (2007) Stimulated ErbB4 internalization is necessary for neuregulin signaling in neurons. Biochem Biophys Res Commun 354:505-510.

Longart M, Chatani-Hinze M, Gonzalez CM, Vullhorst D, Buonanno A (2007) Regulation of ErbB-4 endocytosis by neuregulin in GABAergic hippocampal interneurons. Brain Res Bull 73:210-219.

López-Bendito G, Cautinat A, Sánchez JA, Bielle F, Flames N, Garratt AN, Talmage DA, Role LW, Charnay P, Marin O, Garel S (2006) Tangential neuronal migration controls axon guidance: a role for neuregulin-1 in thalamocortical axon navigation. Cell 125:127-142.

LOWRY OH, ROSEBROUGH NJ, FARR AL, RANDALL RJ (1951) Protein measurement with the Folin phenol reagent. J Biol Chem 193:265-275.

Luzón-Toro B, Torroglosa A, Núñez-Torres R, Enguix-Riego MV, Fernández RM, de Agustín JC, Antiñolo G, Borrego S (2012) Comprehensive analysis of NRG1 common and rare variants in Hirschsprung patients. PLoS One 7:e36524.

Ma L, Huang YZ, Pitcher GM, Valtschanoff JG, Ma YH, Feng LY, Lu B, Xiong WC, Salter MW, Weinberg RJ, Mei L (2003) Ligand-dependent recruitment of the ErbB4 signaling complex into neuronal lipid rafts. J Neurosci 23:3164-3175.

Madisen L, Zwingman TA, Sunkin SM, Oh SW, Zariwala HA, Gu H, Ng LL, Palmiter RD, Hawrylycz MJ, Jones AR, Lein ES, Zeng H (2010) A robust and high-throughput Cre reporting and characterization system for the whole mouse brain. Nat Neurosci 13:133140.

Maekawa M, Ishizaki T, Boku S, Watanabe N, Fujita A, Iwamatsu A, Obinata T, Ohashi K, Mizuno K, Narumiya S (1999) Signaling from Rho to the actin cytoskeleton through protein kinases ROCK and LIM-kinase. Sci (New York, NY) 285:895-898.

Marchionni MA, Goodearl AD, Chen MS, Bermingham-McDonogh O, Kirk C, Hendricks M, Danehy F, Misumi D, Sudhalter J, Kobayashi K (1993) Glial growth factors are alternatively spliced erbB2 ligands expressed in the nervous system. Nature 362:312318.

Marin O (2012) Interneuron dysfunction in psychiatric disorders. Nat Rev Neurosci 13:107120. 
Marin O (2013) Cellular and molecular mechanisms controlling the migration of neocortical interneurons. Eur J Neurosci 38:2019-2029.

Marin O, Müller U (2014) Lineage origins of GABAergic versus glutamatergic neurons in the neocortex. Curr Opin Neurobiol 26C:132-141.

Markram H, Toledo-Rodriguez M, Wang Y, Gupta A, Silberberg G, Wu C (2004) Interneurons of the neocortical inhibitory system. Nat Rev Neurosci 5:793-807.

Mata I, Perez-Iglesias R, Roiz-Santiañez R, Tordesillas-Gutierrez D, Gonzalez-Mandly A, Vazquez-Barquero JL, Crespo-Facorro B (2009) A neuregulin 1 variant is associated with increased lateral ventricle volume in patients with first-episode schizophrenia. Biol Psychiatry 65:535-540.

McGuffin P, Owen MJ, Gottesman II (2004) Psychiatric Genetics and Genomics. OUP Oxford.

McIntyre E, Blackburn E, Brown PJ, Johnson CG, Gullick WJ (2010) The complete family of epidermal growth factor receptors and their ligands are co-ordinately expressed in breast cancer. Breast Cancer Res Treat 122:105-110.

McKenna PJ (2013) Schizophrenia and Related Syndromes. Taylor \& Francis.

Mei L, Nave K-A (2014) Neuregulin-ERBB Signaling in the Nervous System and Neuropsychiatric Diseases. Neuron 83:27-49.

Mei L, Xiong W-C (2008) Neuregulin 1 in neural development, synaptic plasticity and schizophrenia. Nat Rev Neurosci 9:437-452.

Meng Y, Zhang Y, Tregoubov V, Falls DL, Jia Z (2003) Regulation of spine morphology and synaptic function by LIMK and the actin cytoskeleton. Rev Neurosci 14:233-240.

Meng Y, Zhang Y, Tregoubov V, Janus C, Cruz L, Jackson M, Lu WY, MacDonald JF, Wang JY, Falls DL, Jia Z (2002) Abnormal spine morphology and enhanced LTP in LIMK-1 knockout mice. Neuron 35:121-133.

Metzger D, Chambon P (2001) Site- and time-specific gene targeting in the mouse. Methods (San Diego, Calif) 24:71-80.

Meyer AH, Katona I, Blatow M, Rozov A, Monyer H (2002) In vivo labeling of parvalbuminpositive interneurons and analysis of electrical coupling in identified neurons. $J$ Neurosci 22:7055-7064.

Meyer D, Yamaai T, Garratt A, Riethmacher-Sonnenberg E, Kane D, Theill LE, Birchmeier C (1997) Isoform-specific expression and function of neuregulin. Development 124:35753586.

Michailov G V, Sereda MW, Brinkmann BG, Fischer TM, Haug B, Birchmeier C, Role LW, Lai C, Schwab MH, Nave K-A (2004) Axonal neuregulin-1 regulates myelin sheath thickness. Science (80- ) 304:700-703.

Millet V, Marder M, Pasquini LA (2012) Adult CNP::EGFP transgenic mouse shows pronounced hypomyelination and an increased vulnerability to cuprizone-induced demyelination. Exp Neurol 233:490-504.

Minichiello L, Korte M, Wolfer D, Kühn R, Unsicker K, Cestari V, Rossi-Arnaud C, Lipp HP, Bonhoeffer T, Klein R (1999) Essential role for TrkB receptors in hippocampus-mediated learning. Neuron 24:401-414.

Mizuno K (2012) Signaling mechanisms and functional roles of cofilin phosphorylation and dephosphorylation. Cell Signal.

Moyer CE, Shelton MA, Sweet RA (2014) Dendritic spine alterations in schizophrenia. Neurosci Lett.

Mullis K, Faloona F, Scharf S, Saiki R, Horn G, Erlich H (1986) Specific enzymatic amplification of DNA in vitro: the polymerase chain reaction. Cold Spring Harb Symp Quant Biol 51 Pt 1:263-273.

Neddens J, Buonanno A (2009) Selective populations of hippocampal interneurons express ErbB4 and their number and distribution is altered in ErbB4 knockout mice. Hippocampus. 
Neddens J, Buonanno A (2011) Expression of the neuregulin receptor ErbB4 in the brain of the rhesus monkey (Macaca mulatta). PLoS One 6:e27337.

Neddens J, Fish KN, Tricoire L, Vullhorst D, Shamir A, Chung W, Lewis DA, McBain CJ, Buonanno A (2011) Conserved interneuron-specific ErbB4 expression in frontal cortex of rodents, monkeys, and humans: implications for schizophrenia. Biol Psychiatry 70:636-645.

Ni CY, Murphy MP, Golde TE, Carpenter G (2001) gamma -Secretase cleavage and nuclear localization of ErbB-4 receptor tyrosine kinase. Science (80- ) 294:2179-2181.

Nicodemus KK, Law AJ, Radulescu E, Luna A, Kolachana B, Vakkalanka R, Rujescu D, Giegling I, Straub RE, McGee K, Gold B, Dean M, Muglia P, Callicott JH, Tan H-Y, Weinberger DR (2010) Biological validation of increased schizophrenia risk with NRG1, ERBB4, and AKT1 epistasis via functional neuroimaging in healthy controls. Arch Gen Psychiatry 67:991-1001.

Nicodemus KK, Luna A, Vakkalanka R, Goldberg T, Egan M, Straub RE, Weinberger DR (2006) Further evidence for association between ErbB4 and schizophrenia and influence on cognitive intermediate phenotypes in healthy controls. Mol Psychiatry 11:1062-1065.

Niwa R, Nagata-Ohashi K, Takeichi M, Mizuno K, Uemura T (2002) Control of actin reorganization by Slingshot, a family of phosphatases that dephosphorylate ADF/cofilin. Cell 108:233-246.

Norton N, Moskvina V, Morris DW, Bray NJ, Zammit S, Williams NM, Williams HJ, Preece AC, Dwyer S, Wilkinson JC, Spurlock G, Kirov G, Buckland P, Waddington JL, Gill M, Corvin AP, Owen MJ, O'Donovan MC (2006a) Evidence that interaction between neuregulin 1 and its receptor erbB4 increases susceptibility to schizophrenia. Am J Med Genet Part B, Neuropsychiatr Genet Off Publ Int Soc Psychiatr Genet 141B:96-101.

Norton N, Williams HJ, Owen MJ (2006b) An update on the genetics of schizophrenia. Curr Opin Psychiatry 19:158-164.

O'Callaghan JP, Holtzman SG (1975) Quantification of the analgesic activity of narcotic antagonists by a modified hot-plate procedure. J Pharmacol Exp Ther 192:497-505.

Okada M, Corfas G (2004) Neuregulin1 downregulates postsynaptic GABAA receptors at the hippocampal inhibitory synapse. Hippocampus 14:337-344.

Omerovic J, Puggioni EMR, Napoletano S, Visco V, Fraioli R, Frati L, Gulino A, Alimandi M (2004) Ligand-regulated association of ErbB-4 to the transcriptional co-activator YAP65 controls transcription at the nuclear level. Exp Cell Res 294:469-479.

Ongür D, Prescot AP, McCarthy J, Cohen BM, Renshaw PF (2010) Elevated gammaaminobutyric acid levels in chronic schizophrenia. Biol Psychiatry 68:667-670.

Owen MJ, Craddock N, O'Donovan MC (2005) Schizophrenia: genes at last? Trends Genet 21:518-525.

Penzes P, Cahill ME, Jones KA, Vanleeuwen J-E, Woolfrey KM (2011) Dendritic spine pathology in neuropsychiatric disorders. Nat Neurosci 14:285-293.

Penzes P, Jones KA (2008) Dendritic spine dynamics--a key role for kalirin-7. Trends Neurosci 31:419-427.

Peterson GL (1979) Review of the Folin phenol protein quantitation method of Lowry, Rosebrough, Farr and Randall. Anal Biochem 100:201-220.

Phusantisampan T, Sangkhathat S, Phongdara A, Chiengkriwate P, Patrapinyokul S, Mahasirimongkol $S$ (2012) Association of genetic polymorphisms in the RETprotooncogene and NRG1 with Hirschsprung disease in Thai patients. J Hum Genet 57:286-293.

Pinkas-Kramarski R, Soussan L, Waterman H, Levkowitz G, Alroy I, Klapper L, Lavi S, Seger R, Ratzkin BJ, Sela M, Yarden Y (1996) Diversification of Neu differentiation factor and epidermal growth factor signaling by combinatorial receptor interactions. EMBO J 15:2452-2467. 
Pirotte D, Wislet-Gendebien S, Cloes JM, Rogister B (2010) Neuregulin-1 modulates the differentiation of neural stem cells in vitro through an interaction with the Swi/Snf complex. Mol Cell Neurosci 43:72-80.

Pitcher GM, Beggs S, Woo R-S, Mei L, Salter MW (2008) ErbB4 is a suppressor of long-term potentiation in the adult hippocampus. Neuroreport 19:139-143.

Plani-Lam JH-C, Chow T-C, Siu K-L, Chau WH, Ng M-HJ, Bao S, Ng CT, Sham P, Shum DKY, Ingley E, Jin D-Y, Song Y-Q (2015) PTPN21 exerts pro-neuronal survival and neuritic elongation via ErbB4/NRG3 signaling. Int J Biochem Cell Biol 61:53-62.

Poliak S, Peles E (2003) The local differentiation of myelinated axons at nodes of Ranvier. Nat Rev Neurosci 4:968-980.

Rio C, Buxbaum JD, Peschon JJ, Corfas G (2000) Tumor necrosis factor-alpha-converting enzyme is required for cleavage of erbB4/HER4. J Biol Chem 275:10379-10387.

Role LW, Talmage DA (2007) Neurobiology: new order for thought disorders. Nature 448:263-265.

Saab AS, Tzvetanova ID, Nave K-A (2013) The role of myelin and oligodendrocytes in axonal energy metabolism. Curr Opin Neurobiol 23:1065-1072.

Salzer JL (2003) Polarized domains of myelinated axons. Neuron 40:297-318.

Sanes JR (1994) Lineage tracing. The laatest in lineaage. Curr Biol 4:1162-1164.

Santello M, Volterra A (2009) Synaptic modulation by astrocytes via Ca2+-dependent glutamate release. Neuroscience 158:253-259.

Sardi SP, Murtie J, Koirala S, Patten BA, Corfas G (2006) Presenilin-dependent ErbB4 nuclear signaling regulates the timing of astrogenesis in the developing brain. Cell 127:185-197.

Sarmiere PD, Bamburg JR (2002) Head, neck, and spines: a role for LIMK-1 in the hippocampus. Neuron 35:3-5.

Savonenko A V, Melnikova T, Laird FM, Stewart K-A, Price DL, Wong PC (2008) Alteration of BACE1-dependent NRG1/ErbB4 signaling and schizophrenia-like phenotypes in BACE1-null mice. Proc Natl Acad Sci U S A 105:5585-5590.

Sawyer C, Hiles I, Page M, Crompton M, Dean C (1998) Two erbB-4 transcripts are expressed in normal breast and in most breast cancers. Oncogene 17:919-924.

Schulze WX, Deng L, Mann M (2005) Phosphotyrosine interactome of the ErbB-receptor kinase family. Mol Syst Biol 1:2005.0008.

Schwab MH, Druffel-Augustin S, Gass P, Jung M, Klugmann M, Bartholomae A, Rossner MJ, Nave K-A (1998) Neuronal basic helix-loop-helix proteins (NEX, neuroD, NDRF): spatiotemporal expression and targeted disruption of the NEX gene in transgenic mice. J Neurosci 18:1408-1418.

Scott DJ, Heitzeg MM, Koeppe RA, Stohler CS, Zubieta J-K (2006) Variations in the human pain stress experience mediated by ventral and dorsal basal ganglia dopamine activity. J Neurosci 26:10789-10795.

Scott RW, Olson MF (2007) LIM kinases: function, regulation and association with human disease. J Mol Med 85:555-568.

Sham PC, MacLean CJ, Kendler KS (1994) A typological model of schizophrenia based on age at onset, sex and familial morbidity. Acta Psychiatr Scand 89:135-141.

Shamir A, Kwon O-B, Karavanova I, Vullhorst D, Leiva-Salcedo E, Janssen MJ, Buonanno A (2012) The Importance of the NRG-1/ErbB4 Pathway for Synaptic Plasticity and Behaviors Associated with Psychiatric Disorders. J Neurosci 32:2988-2997.

Si J, Wang Q, Mei L (1999) Essential roles of c-JUN and c-JUN N-terminal kinase (JNK) in neuregulin-increased expression of the acetylcholine receptor epsilon-subunit. $\mathrm{J}$ Neurosci 19:8498-8508.

Silberberg G, Darvasi A, Pinkas-Kramarski R, Navon R (2006) The involvement of ErbB4 with schizophrenia: association and expression studies. Am J Med Genet Part B, Neuropsychiatr Genet Off Publ Int Soc Psychiatr Genet 141B:142-148. 
Snodgrass-Belt P, Gilbert JL, Davis FC (2005) Central administration of transforming growth factor-alpha and neuregulin-1 suppress active behaviors and cause weight loss in hamsters. Brain Res 1038:171-182.

Snyder SH (1973) Amphetamine psychosis: a "model" schizophrenia mediated by catecholamines. Am J Psychiatry 130:61-67.

Somogyi P (1977) A specific "axo-axonal" interneuron in the visual cortex of the rat. Brain Res 136:345-350.

Soriano P (1999) Generalized lacZ expression with the ROSA26 Cre reporter strain. Nat Genet 21:70-71.

Sparrow N, Manetti ME, Bott M, Fabianac T, Petrilli A, Bates ML, Bunge MB, Lambert S, Fernandez-Valle $C$ (2012) The actin-severing protein cofilin is downstream of neuregulin signaling and is essential for schwann cell myelination. J Neurosci 32:5284-5297.

Spratley SJ, Bastea LI, Döppler H, Mizuno K, Storz P (2011) Protein kinase D regulates cofilin activity through p21-activated kinase 4 . J Biol Chem 286:34254-34261.

Stefansson H et al. (2002) Neuregulin 1 and susceptibility to schizophrenia. Am J Hum Genet 71:877-892.

Stefansson H, Sarginson J, Kong A, Yates P, Steinthorsdottir V, Gudfinnsson E, Gunnarsdottir S, Walker N, Petursson H, Crombie C, Ingason A, Gulcher JR, Stefansson K, St Clair D (2003) Association of neuregulin 1 with schizophrenia confirmed in a Scottish population. Am J Hum Genet 72:83-87.

Steinthorsdottir V, Stefansson H, Ghosh S, Birgisdottir B, Bjornsdottir S, Fasquel AC, Olafsson O, Stefansson K, Gulcher JR (2004) Multiple novel transcription initiation sites for NRG1. Gene 342:97-105.

Swerdlow NR, Geyer MA (1998) Using an animal model of deficient sensorimotor gating to study the pathophysiology and new treatments of schizophrenia. Schizophr Bull 24:285301.

Swerdlow NR, Weber M, Qu Y, Light GA, Braff DL (2008) Realistic expectations of prepulse inhibition in translational models for schizophrenia research. Psychopharmacology (Berl) 199:331-388.

Taepavarapruk P, Floresco SB, Phillips AG (2000) Hyperlocomotion and increased dopamine efflux in the rat nucleus accumbens evoked by electrical stimulation of the ventral subiculum: role of ionotropic glutamate and dopamine D1 receptors. Psychopharmacology (Berl) 151:242-251.

Talmage DA (2008) Mechanisms of neuregulin action. Novartis Found Symp 289:74-93.

Tan G-H, Liu Y-Y, Hu X-L, Yin D-M, Mei L, Xiong Z-Q (2011) Neuregulin 1 represses limbic epileptogenesis through ErbB4 in parvalbumin-expressing interneurons. Nat Neurosci.

Tang CS-M, Ngan ES-W, Tang W-K, So M-T, Cheng G, Miao X-P, Leon TY-Y, Leung BM-C, Hui K-JWS, Lui VH-C, Chen Y, Chan IH-Y, Chung PH-Y, Liu X-L, Wong KK-Y, Sham PC, Cherny SS, Tam PK-H, Garcia-Barcelo M-M (2012a) Mutations in the NRG1 gene are associated with Hirschsprung disease. Hum Genet 131:67-76.

Tang CS-M, Tang W-K, So M-T, Miao X-P, Leung BM-C, Yip BH-K, Leon TY-Y, Ngan ES-W, Lui VC-H, Chen Y, Chan IH-Y, Chung PH-Y, Liu X-L, Wu X-Z, Wong KK-Y, Sham P-C, Cherny SS, Tam PK-H, Garcia-Barceló M-M (2011) Fine mapping of the NRG1 Hirschsprung's disease locus. PLoS One 6:e16181.

Tang W, Li B, Xu X, Zhou Z, Wu W, Tang J, Qin J, Geng Q, Jiang W, Zhang J, Sha J, Xia Y, Wang $X(2012 b)$ Aberrant high expression of NRG1 gene in Hirschsprung disease. $J$ Pediatr Surg 47:1694-1698.

Tidcombe H, Jackson-Fisher A, Mathers K, Stern DF, Gassmann M, Golding JP (2003) Neural and mammary gland defects in ErbB4 knockout mice genetically rescued from embryonic lethality. Proc Natl Acad Sci U S A 100:8281-8286. 
Ting AK, Chen Y, Wen L, Yin D-M, Shen C, Tao Y, Liu X, Xiong W-C, Mei L (2011) Neuregulin 1 promotes excitatory synapse development and function in GABAergic interneurons. J Neurosci 31:15-25.

Towbin H, Staehelin T, Gordon JA (1979) Electrophoretic transfer of proteins from polyacrylamide gels to nitrocellulose sheets: procedure and some applications. Proc Natl Acad Sci U S A 76:4350-4354.

Vecchi M, Baulida J, Carpenter G (1996) Selective cleavage of the heregulin receptor ErbB-4 by protein kinase $C$ activation. J Biol Chem 271:18989-18995.

Velanac V, Unterbarnscheidt T, Hinrichs W, Gummert MN, Fischer TM, Rossner MJ, Trimarco A, Brivio V, Taveggia C, Willem M, Haass C, Möbius W, Nave K-A, Schwab MH (2011) Bace1 processing of NRG1 type III produces a myelin-inducing signal but is not essential for the stimulation of myelination. Glia.

Vogels TP, Abbott LF (2009) Gating multiple signals through detailed balance of excitation and inhibition in spiking networks. Nat Neurosci 12:483-491.

Voytas D (2001) Agarose gel electrophoresis. Curr Protoc Immunol / Ed by John E Coligan [et al] Chapter 10:Unit 10.4.

Vullhorst D, Neddens J, Karavanova I, Tricoire L, Petralia RS, McBain CJ, Buonanno A (2009) Selective expression of ErbB4 in interneurons, but not pyramidal cells, of the rodent hippocampus. J Neurosci 29:12255-12264.

Wallasch C, Weiss FU, Niederfellner G, Jallal B, Issing W, Ullrich A (1995) Heregulindependent regulation of HER2/neu oncogenic signaling by heterodimerization with HER3. EMBO J 14:4267-4275.

Walss-Bass C, Liu W, Lew DF, Villegas R, Montero P, Dassori A, Leach RJ, Almasy L, Escamilla M, Raventos H (2006) A novel missense mutation in the transmembrane domain of neuregulin 1 is associated with schizophrenia. Biol Psychiatry 60:548-553.

Wang JY, Frenzel KE, Wen D, Falls DL (1998) Transmembrane neuregulins interact with LIM kinase 1, a cytoplasmic protein kinase implicated in development of visuospatial cognition. J Biol Chem 273:20525-20534.

Weickert CS, Tiwari Y, Schofield PR, Mowry BJ, Fullerton JM (2012) Schizophreniaassociated HapICE haplotype is associated with increased NRG1 type III expression and high nucleotide diversity. Transl Psychiatry 2:e104.

Wen D, Peles E, Cupples R, Suggs S V, Bacus SS, Luo Y, Trail G, Hu S, Silbiger SM, Levy RB (1992) Neu differentiation factor: a transmembrane glycoprotein containing an EGF domain and an immunoglobulin homology unit. Cell 69:559-572.

Wen D, Suggs S V, Karunagaran D, Liu N, Cupples RL, Luo Y, Janssen AM, Ben-Baruch N, Trollinger DB, Jacobsen VL (1994) Structural and functional aspects of the multiplicity of Neu differentiation factors. Mol Cell Biol 14:1909-1919.

Wen L, Lu Y-S, Zhu X-H, Li X-M, Woo R-S, Chen Y-J, Yin D-M, Lai C, Terry A V, Vazdarjanova A, Xiong W-C, Mei L (2010) Neuregulin 1 regulates pyramidal neuron activity via ErbB4 in parvalbumin-positive interneurons (Suppl. Material). Proc Natl Acad Sci U S A 107:1211-1216.

Whittaker VP (1993) Thirty years of synaptosome research. J Neurocytol 22:735-742.

Wieser GL, Gerwig UC, Adamcio B, Barrette B, Nave K-A, Ehrenreich H, Goebbels S (2013) Neuroinflammation in white matter tracts of Cnp1 mutant mice amplified by a minor brain injury. Glia 61:869-880.

Willem M, Garratt AN, Novak B, Citron M, Kaufmann S, Rittger A, DeStrooper B, Saftig P, Birchmeier C, Haass C (2006) Control of peripheral nerve myelination by the betasecretase BACE1. Science (80- ) 314:664-666.

Willem M, Lammich S, Haass C (2009) Function, regulation and therapeutic properties of beta-secretase (BACE1). Semin Cell Dev Biol 20:175-182. 
Williams CC, Allison JG, Vidal GA, Burow ME, Beckman BS, Marrero L, Jones FE (2004) The ERBB4/HER4 receptor tyrosine kinase regulates gene expression by functioning as a STAT5A nuclear chaperone. J Cell Biol 167:469-478.

Wolpowitz D, Mason TB, Dietrich P, Mendelsohn M, Talmage DA, Role LW (2000) Cysteinerich domain isoforms of the neuregulin-1 gene are required for maintenance of peripheral synapses. Neuron 25:79-91.

Woo R-S, Li X-M, Tao Y, Carpenter-Hyland E, Huang YZ, Weber JL, Neiswender H, Dong XP, Wu J, Gassmann M, Lai C, Xiong W-C, Gao T-M, Mei L (2007) Neuregulin-1 enhances depolarization-induced GABA release. Neuron 54:599-610.

Wu GY, Deisseroth K, Tsien RW (2001) Spaced stimuli stabilize MAPK pathway activation and its effects on dendritic morphology. Nat Neurosci 4:151-158.

Yang JZ, Si TM, Ruan Y, Ling YS, Han YH, Wang XL, Zhou M, Zhang HY, Kong QM, Liu C, Zhang DR, Yu YQ, Liu SZ, Ju GZ, Shu L, Ma DL, Zhang D (2003) Association study of neuregulin 1 gene with schizophrenia. Mol Psychiatry 8:706-709.

Yang N, Higuchi O, Ohashi K, Nagata K, Wada A, Kangawa K, Nishida E, Mizuno K (1998) Cofilin phosphorylation by LIM-kinase 1 and its role in Rac-mediated actin reorganization. Nature 393:809-812.

Yang N, Mizuno K (1999) Nuclear export of LIM-kinase 1, mediated by two leucine-rich nuclear-export signals within the PDZ domain. Biochem J 338 ( Pt 3:793-798.

Yang X-L, Huang YZ, Xiong WC, Mei L (2005) Neuregulin-induced expression of the acetylcholine receptor requires endocytosis of ErbB receptors. Mol Cell Neurosci 28:335-346.

Yarden Y, Sliwkowski MX (2001) Untangling the ErbB signalling network. Nat Rev Mol cell Biol 2:127-137.

Yin D-M, Chen Y-J, Lu Y-S, Bean JC, Sathyamurthy A, Shen C, Liu X, Lin TW, Smith CA, Xiong W-C, Mei L (2013a) Reversal of behavioral deficits and synaptic dysfunction in mice overexpressing neuregulin 1. Neuron 78:644-657.

Yin D-M, Sun X-D, Bean JC, Lin TW, Sathyamurthy A, Xiong W-C, Gao T-M, Chen Y-J, Mei L (2013b) Regulation of Spine Formation by ErbB4 in PV-Positive Interneurons. J Neurosci 33:19295-19303.

Yokozeki T, Wakatsuki S, Hatsuzawa K, Black RA, Wada I, Sehara-Fujisawa A (2007) Meltrin beta (ADAM19) mediates ectodomain shedding of Neuregulin beta1 in the Golgi apparatus: fluorescence correlation spectroscopic observation of the dynamics of ectodomain shedding in living cells. Genes Cells 12:329-343.

Yoon JH, Maddock RJ, Rokem A, Silver MA, Minzenberg MJ, Ragland JD, Carter CS (2010) GABA concentration is reduced in visual cortex in schizophrenia and correlates with orientation-specific surround suppression. J Neurosci 30:3777-3781.

Young JW, Wallace CK, Geyer MA, Risbrough VB (2010) Age-associated improvements in cross-modal prepulse inhibition in mice. Behav Neurosci 124:133-140.

Zhang D, Sliwkowski MX, Mark M, Frantz G, Akita R, Sun Y, Hillan K, Crowley C, Brush J, Godowski PJ (1997) Neuregulin-3 (NRG3): a novel neural tissue-enriched protein that binds and activates ErbB4. Proc Natl Acad Sci U S A 94:9562-9567.

Zhou W, Carpenter G (2000) Heregulin-dependent trafficking and cleavage of ErbB-4. J Biol Chem 275:34737-34743.

Zhu JJ, Esteban JA, Hayashi Y, Malinow R (2000) Postnatal synaptic potentiation: delivery of GluR4-containing AMPA receptors by spontaneous activity. Nat Neurosci 3:1098-1106.

Ziff EB (1997) Enlightening the postsynaptic density. Neuron 19:1163-1174. 


\section{Curriculum vitae}

First Name, Surname:

Date of birth:

Place of birth:

Nationality:

Home address:
Tilmann Unterbarnscheidt

11. February 1984

Essen (an der Ruhr), Germany

German

Arnswaldtstr. 4

30159 Hannover, Germany

\section{Education:}

02/2015 - present

$02 / 2012-04 / 2015$

09/2010 - 12/2011

$05 / 2010$

$03 / 2008-01 / 2009$

Research associate at the Department of Cellular Neurophysiology, Center of Physiology, Hannover Medical School (Research group of Dr. Markus $\mathrm{H}$. Schwab).

Graduate student at the Max-Planck-Institute of Experimental Medicine (Göttingen), Department of Neurogenetics (Prof. Klaus-Armin Nave, Ph.D.); Thesis: "Conditional activation of NRG1 signaling in the brain modulates cortical circuitry”, (Research group "Cortical Development' of Dr. Markus H. Schwab).

Research associate with a stipend of the Max-PlanckSociety at the Max-Planck-Institute of Experimental Medicine (Göttingen), Department of Neurogenetics (Prof. Klaus-Armin Nave, Ph.D.); Topic: "Comparative analysis of brain development in NRG1 transgenic mouse lines on histological, biochemical and functional level', (Research group 'Cortical Development' of Dr. Markus H. Schwab).

Diploma of biology from the Georg-August-University of Göttingen.

Diploma thesis at the Max-Planck-Institute of Experimental Medicine (Göttingen), Department of Neurogenetics (Prof. Klaus-Armin Nave, Ph.D.), entitled: "Untersuchung zur Funktion, Prozessierung und Lokalisation von NRG1 type III-Varianten im ZNS transgener Mauslinien”, (Research group “Cortical Development' of Dr. Markus H. Schwab).

Student research assistent at the Max-Planck-Institute of Experimental Medicine (Göttingen), Department of Neurogenetics (Prof. Klaus-Armin Nave, Ph.D.) in the research group 'Cortical Development' of Dr. Markus H. 
Schwab.

$10 / 2006-09 / 2010$

$10 / 2004-09 / 2006$

2003
Academic study of biology at the Georg-AugustUniversity of Göttingen.

Subjects: Zoology, biochemistry and psychology.

Academic study of biology at the Ruhr University Bochum and prediploma of biology.

Allgemeinen Hochschulreife (Abitur) from the Gymnasium Essen-Werden, Germany.

\section{Publications:}

Wehr MC, Hinrichs W, Brzózka MM, Soto-Bernardini MC, Unterbarnscheidt T, Zhang M, Wichert SP, Falkai P, Zhang W, Schwab MH, Rossner MJ. (2015). Spironolactone is an inhibitor of NRG1-ErbB4 signalling and ameliorates schizophrenia-relevant endophenotypes in NRG1 transgenic mice. In preparation.

Brzózka MM, Unterbarnscheidt T, Schwab MH, Rossner MJ. (2015). OSO paradigm - a rapid behavioral screening method for acute psychosocial stress reactivity in mice. In Preparation.

Pieper A, Rudolph S, Wieser G, Unterbarnscheidt T, Yonemasu T, Yan K, Weege B, Bode U, Bormuth I, Wadiche JI, Schwab MH, Goebbels S. (2015). Control of inhibitory circuit formation in the cerebellum by NeuroD2. Submitted to The Journal of Neuroscience.

Agarwal $A^{*}$, Zhang $M^{*}$, Trembak-Duff $I^{*}$, Unterbarnscheidt $\mathbf{T}^{*}$, et al. (2014). Dysregulated Expression of Neuregulin-1 by Cortical Pyramidal Neurons Disrupts Synaptic Plasticity. Cell Reports. 2014 Aug 21;8(4):1130-45.

*equally contributing first author

Heermann S, Schmücker J, Hinz U, Rickmann M, Unterbarnscheidt T, Schwab MH, Krieglstein K. (2011). Neuregulin 1 type III/ErbB signaling is crucial for Schwann cell colonization of sympathetic axons. PLoS One. 2011;6(12):e28692.

Velanac V, Unterbarnscheidt T, Hinrichs W, Gummert MN, Fischer TM, Rossner MJ, Trimarco A, Brivio V, Taveggia C, Willem M, Haass C, Möbius W, Nave KA, Schwab MH. (2012). Bace1 processing of NRG1 type III produces a myelin-inducing signal but is not essential for the stimulation of myelination. Glia. 2012 Feb;60(2):203-17. 


\section{Lebenslauf}

Vorname, Name:

Tilmann Unterbarnscheidt

Geburtstag:

11. Februar 1984

Geburtsort:

Staatsangehörigkeit:

Anschrift:
Essen (an der Ruhr), Deutschland

Deutsch

Arnswaldtstr. 4

30159 Hannover, Deutschland

\section{Ausbildung:}

Seit 02/2015

02/2012-04/2015

09/2010 - 12/2011

$05 / 2010$

03/2008 - 01/2009

Wissenschaftlicher Mitarbeiter in der Abteilung für Zelluläre Neurophysiologie, Zentrum für Physiologie, Medizinische Hochschule Hannover (Arbeitsgruppe von Markus H. Schwab).

Doktorand am Max-Planck-Institut für Experimentelle Medizin (Göttingen), Abteilung Neurogenetik (Prof. Klaus-Armin Nave, Ph.D.); Thema: "Conditional activation of NRG1 signaling in the brain modulates cortical circuitry", (Forschungsgruppe 'Kortex Entwicklung' von Dr. Markus H. Schwab).

Wissenschaftlicher Mitarbeiter mit Stipendium der MaxPlanck-Gesellschaft am Max-Planck-Institut für Experimentelle Medizin (Göttingen), Abteilung Neurogenetik (Prof. Klaus-Armin Nave, Ph.D.); Thema: "Vergleichende Analyse der Gehirnentwicklung bei NRG1 transgenen Mauslinien auf histologischer, biochemischer und funktionaler Ebene", (Forschungsgruppe 'Kortex Entwicklung' von Dr. Markus H. Schwab).

Diplom in Biologie an der Georg-August-Universität Göttingen.

Externe Diplomarbeit am Max-Planck-Institut für Experimentelle Medizin (Göttingen), Abteilung Neurogenetik (Prof. Klaus-Armin Nave, Ph.D.); Titel: "Untersuchung zur Funktion, Prozessierung und Lokalisation von NRG1 type III-Varianten im ZNS transgener Mauslinien”, (Forschungsgruppe 'Kortex Entwicklung' von Dr. Markus H. Schwab).

Studentische Hilfskraft (,Hiwi') am Max-Planck-Institut für Experimentelle Medizin (Göttingen), Abteilung Neurogenetik (Prof. Klaus-Armin Nave, Ph.D.) in der 
Forschungsgruppe 'Kortex Entwicklung' von Dr. Markus H. Schwab.

10/2006-09/2010 Hauptstudium im Diplomstudiengang Biologie an der Georg-August-Universität Göttingen.

Fächer: Zoologie, Biochemie und Psychologie.

10/2004-09/2006 Grundstudium im Diplomstudiengang Biologie an der Ruhr-Universität-Bochum, Abschluss mit Vordiplom in Biologie.

2003

Allgemeinen Hochschulreife (Abitur) am Gymnasium Essen-Werden, Deutschland.

\section{Publikationen:}

Wehr MC, Hinrichs W, Brzózka MM, Soto-Bernardini MC, Unterbarnscheidt T, Zhang M, Wichert SP, Falkai P, Zhang W, Schwab MH, Rossner MJ. (2015). Spironolactone is an inhibitor of NRG1-ErbB4 signalling and ameliorates schizophrenia-relevant endophenotypes in NRG1 transgenic mice. In Vorbereitung.

Brzózka MM, Unterbarnscheidt T, Schwab MH, Rossner MJ. (2015). OSO paradigm - a rapid behavioral screening method for acute psychosocial stress reactivity in mice. In Vorbereitung.

Pieper A, Rudolph S, Wieser G, Unterbarnscheidt T, Yonemasu T, Yan K, Weege B, Bode U, Bormuth I, Wadiche JI, Schwab MH, Goebbels S. (2015). Control of inhibitory circuit formation in the cerebellum by NeuroD2. Eingereicht bei The Journal of Neuroscience.

Agarwal $A^{*}$, Zhang $M^{*}$, Trembak-Duff $I^{*}$, Unterbarnscheidt $\mathbf{T}^{*}$, et al. (2014). Dysregulated Expression of Neuregulin-1 by Cortical Pyramidal Neurons Disrupts Synaptic Plasticity. Cell Reports. 2014 Aug 21;8(4):1130-45.

*equally contributing first author

Heermann S, Schmücker J, Hinz U, Rickmann M, Unterbarnscheidt T, Schwab MH, Krieglstein K. (2011). Neuregulin 1 type III/ErbB signaling is crucial for Schwann cell colonization of sympathetic axons. PLoS One. 2011;6(12):e28692.

Velanac V, Unterbarnscheidt T, Hinrichs W, Gummert MN, Fischer TM, Rossner MJ, Trimarco A, Brivio V, Taveggia C, Willem M, Haass C, Möbius W, Nave KA, Schwab MH. (2012). Bace1 processing of NRG1 type III produces a myelin-inducing signal but is not essential for the stimulation of myelination. Glia. 2012 Feb;60(2):203-17. 